\title{
Motivating smokers to quit : effectiveness and feasibility of a web-based multiple tailored smoking cessation programme and tailored counselling by practice nurses
}

Citation for published version (APA):

Smit, E. S. (2012). Motivating smokers to quit : effectiveness and feasibility of a web-based multiple tailored smoking cessation programme and tailored counselling by practice nurses. [Doctoral Thesis, Maastricht University]. Datawyse / Universitaire Pers Maastricht. https://doi.org/10.26481/dis.20121003es

Document status and date:

Published: 01/01/2012

DOI:

$10.26481 /$ dis.20121003es

Document Version:

Publisher's PDF, also known as Version of record

Please check the document version of this publication:

- A submitted manuscript is the version of the article upon submission and before peer-review. There can be important differences between the submitted version and the official published version of record. People interested in the research are advised to contact the author for the final version of the publication, or visit the DOI to the publisher's website.

- The final author version and the galley proof are versions of the publication after peer review.

- The final published version features the final layout of the paper including the volume, issue and page numbers.

Link to publication

\footnotetext{
General rights rights.

- You may freely distribute the URL identifying the publication in the public portal. please follow below link for the End User Agreement:

www.umlib.nl/taverne-license

Take down policy

If you believe that this document breaches copyright please contact us at:

repository@maastrichtuniversity.nl

providing details and we will investigate your claim.
}

Copyright and moral rights for the publications made accessible in the public portal are retained by the authors and/or other copyright owners and it is a condition of accessing publications that users recognise and abide by the legal requirements associated with these

- Users may download and print one copy of any publication from the public portal for the purpose of private study or research.

- You may not further distribute the material or use it for any profit-making activity or commercial gain

If the publication is distributed under the terms of Article 25fa of the Dutch Copyright Act, indicated by the "Taverne" license above, 


\section{Motivating smokers to quit}

EFFECTIVENESS AND FEASIBILITY OF A WEB-BASED MULTIPLE

TAILORED SMOKING CESSATION PROGRAMME AND TAILORED

COUNSELLING BY PRACTICE NURSES

Eline Suzanne Smit 


\section{Colofon}

Cover design: Datawyse | Universitaire Pers Maastricht

Cover Illustration: Stock.XCHNG | www.sxc.hu

Photograph: Bilbo Schickenberg | www.bilbos.nl

Production: $\quad$ Datawyse | Universitaire Pers Maastricht

ISBN: $\quad 9789461591661$

(C) Eline Suzanne Smit, 2012

The research presented in this thesis was conducted at the School for Public Health and Primary Care: CAPHRI, Department of Health Promotion, of Maastricht University. CAPHRI participates in the Netherlands School of Primary Care Research CaRe. CAPHRI was classified as 'excellent' by the external evaluation committee of leading international experts that reviewed CAPHRI in December 2010.

The research presented as well as the publication of this thesis were financially supported by the Dutch Cancer Society (project number UM 2007-3834). 
MOTIVATING SMOKERS TO QUIT

\title{
Effectiveness and feasibility of a web-based multiple tailored smoking cessation programme and tailored counselling by practice nurses
}

\author{
PROEFSCHRIFT \\ ter verkrijging van de graad van doctor \\ aan de Universiteit Maastricht \\ op gezag van de Rector Magnificus prof. dr. L.L.G. Soete \\ volgens het besluit van het College van Decanen, \\ in het openbaar te verdedigen \\ op woensdag 3 oktober 2012 om 16.00 uur \\ door
}

Eline Suzanne Smit

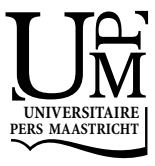




\section{Promotor}

Prof. dr. H. de Vries

\section{Co-promotor}

Dr. C. Hoving

\section{Beoordelingscommissie}

Prof. dr. N.K. de Vries (voorzitter)

Prof. dr. R.A. Knibbe

Prof. dr. Ir. C.P. van Schayck

Prof. dr. T. van der Weijden

Dr. C. Bolman, Open Universiteit Nederland 


\section{CONTENT}

$\begin{array}{lr}\text { CHAPTER } 1 & 7\end{array}$

GENERAL INTRODUCTION

$\begin{array}{ll}\text { CHAPTER } 2 & \mathbf{3 1}\end{array}$

THE PAS STUDY: A RANDOMIZED CONTROLLED TRIAL EVALUATING THE EFFECTIVENESS OF A WEB-BASED MULTIPLE COMPUTER-TAILORED SMOKING CESSATION PROGRAMME AND TAILORED COUNSELLING BY PRACTICE NURSES

\section{CHAPTER 3}

FACTORS INFLUENCING DUTCH PRACTICE NURSES' INTENTION TO ADOPT A NEW SMOKING CESSATION INTERVENTION

\section{CHAPTER 4}

INFLUENCE OF RECRUITMENT STRATEGY ON THE REACH AND EFFECT OF A WEB-BASED MULTIPLE COMPUTER-TAILORED SMOKING CESSATION INTERVENTION AMONGST DUTCH ADULT SMOKERS

\section{CHAPTER 5}

EFFECTIVENESS OF A WEB-BASED MULTIPLE COMPUTER-TAILORED SMOKING CESSATION PROGRAMME: A RANDOMIZED CONTROLLED TRIAL AMONG DUTCH ADULT SMOKERS

\section{CHAPTER 6}

WHO BENEFITS MOST? EFFECTIVENESS OF A WEB-BASED MULTIPLE COMPUTER-TAILORED SMOKING CESSATION PROGRAMME AND TAILORED COUNSELLING BY PRACTICE NURSES ON SMOKING ABSTINENCE REPORTED AFTER SIX AND TWELVE MONTHS

\section{CHAPTER 7}

THE COST-EFFECTIVENESS AND COST-UTILITY OF A WEB-BASED MULTIPLE COMPUTER-TAILORED SMOKING CESSATION PROGRAMME AND TAILORED COUNSELLING BY PRACTICE NURSES: A TRIAL-BASED ECONOMIC EVALUATION

\section{CHAPTER 8}

THE IMPORTANCE OF ATTITUDE AND INNOVATION CHARACTERISTICS IN DETERMINING THE INTENTION TO IMPLEMENT A NEW SMOKING CESSATION INTERVENTION AMONG DUTCH PRACTICE NURSES: A CROSS-SECTIONAL STUDY 
THE ROLE OF DESIRE, DUTY AND INTENTION IN PREDICTING ATTEMPTS TO QUIT SMOKING

CHAPTER 10

DOES A TYPICAL CONTEMPLATOR EXIST? THREE CLUSTERS OF SMOKERS IN CONTEMPLATION

CHAPTER 11

163

GenERAL DisCuSSION

REFERENCES

185

APPENDIX 1

203

APPENDIX 2

205

SUMMARY

207

SAMENVATTING

211

DANKWOORD

215

CURRICULUM VITAE 217

PUBLICATION LIST 
CHAPTER 1

General Introduction 


\section{SMOKING AND SMOKING CESSATION}

The smoking of tobacco has been known as a natural stimulant ever since it was discovered. Nonetheless, already in 1602 the first booklet presenting the negative effects of smoking on health was published (Charlton, 2005). In this booklet, a doctor under the pseudonym of Philaretes discussed many of the health risks of smoking, e.g. the detrimental effects of smoking on people's lungs, fertility and the cardiovascular system, that have found strong support in later research (Charlton, 2005). More than a century later, in 1761, the first clinical study on the effects of tobacco on the human body was conducted (Hill, 1761). Based on the results from this study, Hill concluded that the nasal consumption of tobacco led to increased vulnerability for nasal cancer. Despite this early finding, it took until 1956 for the first major studies on the effects of the smoking of tobacco on health to be conducted (Doll and Hill, 1956, Wynder et al., 1956). Only in 1964, Hill's conclusions were extended from nasal tobacco consumption to smoking (United States Public Health Service, 1964). In the Netherlands, Lenze Meinsma was one of the first researchers studying the health risks of tobacco smoking (Meinsma, 1969); as the director of the Dutch Cancer Society, he started the anti-tobacco lobby in our country. Nowadays, it is well known that the smoking of tobacco is harmful. In fact, smoking is the most harmful way of tobacco consumption (Henningfield and Fagerstrom, 2001). As smoking is an important risk factor for six of the eight leading causes of death, including several types of cancer, cardiovascular diseases and respiratory diseases (WHO, 2008), it is considered the most preventable cause of illness and premature death in the world (USDHHS, 2004, WHO, 2008). Despite the fact that each year 5.4 million people die from the consequences of smoking, more than one billion people in the world continue to smoke (WHO, 2008). In the Netherlands, every year almost 20.000 people die because of smoking-related illnesses (Van Gelder et al., 2011). Nevertheless, still more than 25\% of the Dutch adult population reports to smoke (STIVORO, 2010). To reduce both the number of people suffering from smoking-related illnesses and the number of smoking-related deaths, continued efforts on different levels are required aimed at decreasing the number smokers.

Healthy behaviours, such as smoking cessation, are thought to be maximized when healthful choices are supported by policies and environments (WHO, 1986). Therefore, multiple interventions have been developed and implemented on a policy, or macro-, level as well as on an organizational and community, or meso-, level. On a macro-level, interventions that have been proven effective in decreasing smoking rates are higher cigarette taxes, advertising bans and large media campaigns (Levy et al., 2004, Hopkins et al., 2001, Lemmens et al., 2008). In addition, evidence is accumulating for health warnings on tobacco packages (Hammond, 2011) and health care financing interventions aimed to reduce the costs of providing or using smoking cessation support (Reda et al., 2009). On a meso-level, support has been found for smoking bans in public places such as worksites (Moher et al., 2003, 
Nagelhout et al., 2011, Verdonk-Kleinjan et al., 2011) and bars and restaurants (Nagelhout et al., 2011) as well as for smoking bans in the home setting (Mills et al., 2009). Yet, ensuring a supportive macro- and meso-level environment does not guarantee that individuals will make use of the resources provided and actually change their behaviour (Sallis et al., 2002). This is demonstrated by the finding that still only a minority of the approximately $75 \%$ of Dutch adults smokers who expresses an intention to quit smoking actually undertakes a quit attempt and only few do so successfully (STIVORO, 2010). This suggest that in addition to interventions targeting the macro- and/or meso-level, smoking cessation interventions are warranted that aid the smoker in his attempt to quit on a more individual, or micro-, level. To investigate the effects of micro-level interventions on smoking cessation rates, several systematic reviews have been conducted, which report significant positive effects of nicotine replacement therapy and the antidepressants bupropion and nortriptyline (Lancaster et al., 2000, Lemmens et al., 2008), a brief advice from a physician (Lancaster and Stead, 2008, Lancaster et al., 2000, Stead et al., 2008), individual and group counselling by a physician, smoking cessation specialist or nurse (Lancaster et al., 2000, Lancaster and Stead, 2005b, Lemmens et al., 2008, Rice and Stead, 2008) and of personalized compared to standard self-help materials (Lancaster et al., 2000). Furthermore, a strategy has found support in individual studies (Dijkstra et al., 1998a, Etter and Perneger, 2001b, Te Poel et al., 2009) as well as in different systematic reviews (Krebs et al., 2010, Lustria et al., 2009, Noar et al., 2007, Lemmens et al., 2008) is computer-tailoring. Computertailored interventions adapt intervention materials and provide personalized feedback adjusted to each smoker's individual characteristics (Dijkstra and De Vries, 1999, Velicer and Prochaska, 1999). Compared with non-tailored interventions, tailored interventions are more successful in attracting and keeping the smoker's attention (Kreuter et al., 1999, de Vries and Brug, 1999, Strecher et al., 2008), resulting in more careful processing of information (Dijkstra, 2005).

Also on a micro-level, however, the effects of stand-alone interventions have been suggested to typically be limited (Lancaster et al., 2000). This has put forward the idea that a more comprehensive approach might be needed to result in even larger effects of smoking cessation interventions targeting the individual smoker. Moreover, even for smokers participating in a smoking cessation intervention, a positive motivation to quit smoking is a necessary (Hyland et al., 2006, Norman et al., 1999, Vangeli et al., 2011) though not always sufficient prerequisite for smoking cessation to occur. This might be a result of frequently used measures of motivation overestimating the percentage of smokers truly motivated to quit. To inform the development of future smoking cessation intervention strategies and to ultimately be able to increase smoking abstinence rates, a closer look needs to be taken at the construct of motivation to quit.

The smoking cessation intervention PAS (Personal Advice in Stopping smoking) was developed taking into account the suggestion to adopt a more comprehensive approach, as this intervention combines two previously developed effective 
micro-level interventions: computer-tailoring and behavioural counselling by a practice nurse working in primary care. The present chapter describes the rationale for the development of PAS as well as for the studies conducted to evaluate its effectiveness, cost-effectiveness and feasibility. In addition, the present chapter discusses some of the gaps in our current understanding of the construct of motivation, providing a rationale for the second focus of this dissertation: contributing to an increased understanding of smokers' motivation to quit.

\section{THE I-CHANGE MODEL}

The theoretical framework that was used for the development of PAS, as well as for conducting most of the studies described in this dissertation, is the I-Change Model (ICM) (De Vries et al., 2003) (figure 1.1). As was its predecessor the Attitude-Social influence-Efficacy (ASE) Model (de Vries and Mudde, 1998), the ICM is a theory of behavioural change which incorporates theoretical concepts from several sociocognitive models. These models include the Transtheoretical Model (TTM) (Prochaska et al., 1997), the Theory of Planned Behaviour (TPB) (Ajzen, 1985), Social Cognitive Theory (SCT) (Bandura, 1986) and the Health Belief Model (HBM) (Janz et al., 2002).

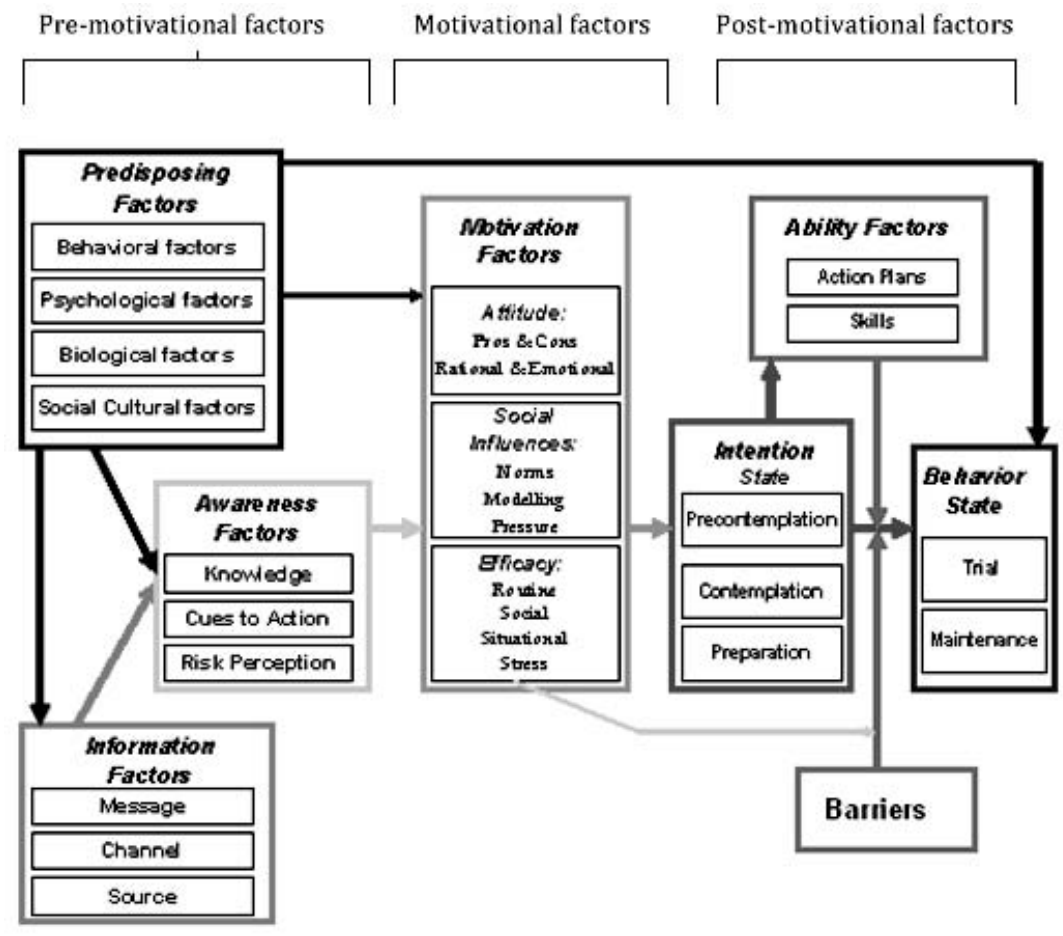

Figure 1.1 The I-Change model (De Vries et al., 2003). 
According to the ICM, the most proximal predictor of behaviour is the intention to perform this behaviour. The ICM subdivides this behavioural intention into three phases using a similar terminology as used in the TTM (Prochaska and DiClemente, 1983): precontemplation, contemplation and preparation (De Vries et al., 2003). According to the ICM these phases can be assessed in two different ways, i.e. in terms of the stages to changes defined by the TTM, which are based on the time period within the person intends to change his or her behaviour (e.g. Hoving et al., 2010, Te Poel et al., 2009), or by using a continuous measure of intention (e.g. van Osch et al., 2010, van Stralen et al., 2010).

The ICM proposes that a person's behavioural intention can be predicted by three motivational constructs: attitude, perceived social influence and self-efficacy. While these constructs are present in many theories of behavioural change, their specific definitions differ across theories. According to the ICM, the concept of attitude entails both the perceived advantages (pros) and disadvantages (cons) of a specific behaviour. Perceived social influence refers to three related but distinct constructs: perceived social norms, social modelling and social pressure. Perceived social norms refer to the perceived opinions held by others in the person's social environment, such as a partner, family and friends. Social modelling refers to perceived behaviour of other people, while social pressure refers to perceived pressure or support from people in the social environment to perform the behaviour. Selfefficacy refers to a person's level of confidence that he can perform the target behaviour. A positive attitude towards smoking cessation, a high self-efficacy to quit, as well as a social environment which is perceived as positive towards smoking cessation are all hypothesized to have a positive influence on the intention to quit smoking. Previous studies that have used the ICM or the ASE Model to study smoking cessation behaviour have indeed identified that these motivational factors are predictive of the intention to quit smoking (Vitoria et al., 2009, Panday et al., 2007, Hoving et al., 2006c, Dijkstra and De Vries, 2000, de Vries and Mudde, 1998).

As suggested by the ICM, the motivational factors attitude, perceived social influence and self-efficacy are predicted by several pre-motivational factors. These pre-motivational factors include predisposing factors, awareness factors and information factors. Predisposing factors consist of behavioural (e.g. acquisition of skills, previous experience with the same and related behaviours), psychological (e.g. attributions, vicarious learning processes), biological (e.g. age, gender, hereditary factors) and socio-cultural factors (e.g. social climate, socio-economic status). Awareness factors entail factors such as knowledge, risk perception and cues to action. Information factors relate to characteristics of the message and to factors associated with the channel and source used to deliver the message (de Vries and Mudde, 1998). The ICM proposes that the pre-motivational factors influence a person's behaviour by influencing attitudes, social influence perceptions, and selfefficacy expectations. Though, as the predisposing factors are considered external factors, additionally these are assumed to have a direct influence on behaviour. Gender, for instance, had been shown to directly influence stress related behaviour 
(Bernstein et al., 2003). While males under stress tend to get angry, avoid stressors, or both, females are more likely to ask for the help of others and to make use of their social support network (Taylor et al., 2000).

In line with earlier findings (e.g. Armitage and Conner, 2001), the ICM recognizes the discrepancy between the intention to change behaviour and actual behavioural change, resulting in the so-called intention-behaviour gap. The ICM recognizes that intention is a necessary prerequisite for behaviour change to occur, but argues that in bridging the gap between intention and behaviour several postmotivational factors play a role. As also suggested by other theorists (e.g. Gollwitzer, 1999, Sniehotta, 2009), perceived barriers to change might increase the gap between intention and behaviour. On the other hand, ability factors such as an individual's skills to refrain from smoking and the formation of action plans are assumed to aid in overcoming these barriers to change and thus to decrease the intention-behaviour gap. According to the ICM, action planning includes preparatory planning and coping planning. With regard to smoking cessation, preparatory planning is assumed to take place in preparation of an attempt to quit smoking. Coping planning is referred to as the planning of strategies aimed to maintain behavioural performance in the face of barriers, such as in situations in which it is perceived as difficult to refrain from smoking. Implemental planning, or implementation intentions, can be used to facilitate the execution of both preparatory and coping plans. Implementation intentions are defined by Gollwitzer (1999) as conditional plans structured like 'if the situation Y occurs, then I will perform the behaviour $x^{\prime}$. Together, preparatory planning and coping planning are argued to increase the likelihood of accomplishing a desired behavioural change. Previous research has found considerable support for the effectiveness of various types of preparatory planning (Hoving et al., 2006b, Scholz et al., 2009, Elfeddali et al., 2012a, De Vries et al., 2012), coping planning (van Osch et al., 2008, O'Connell et al., 2007) and the formulation of implementation intentions (Gollwitzer and Sheeran, 2006, Sniehotta, 2009 ) in increasing the number of quit attempts and rates of smoking abstinence.

\section{THE SMOKING CESSATION INTERVENTION PAS}

The smoking cessation intervention PAS consisted of an Internet-based multiple computer-tailoring programme and tailored counselling by a practice nurse working in Dutch primary care. The rationale for the development of this intervention is described in more detail in the following.

\section{Background}

\section{Smoking cessation activities in the general practice setting}

General practices serve a central role within the Dutch healthcare system. This is illustrated by the fact that in the Netherlands almost $80 \%$ of a general practice's population has at least one consultation with their general practitioner (GP) each year (Schellevis et al., 2005). Furthermore, the general practice has been shown to 
be equally accessible for all socio-demographic subgroups of the Dutch population (Schellevis et al., 2005). As a result, the general practice offers a suitable channel for the implementation of interventions aimed to move the Dutch smoking population towards cessation.

A brief advice by a GP has been shown to result in small though significant effects on smoking cessation rates (Lancaster and Stead, 2008), a finding replicated by several Dutch studies (Pieterse et al., 2001, Hilberink et al., 2011). Hence, guidelines have been developed to facilitate the implementation of smoking cessation activities in the Dutch general practice setting, such as the Dutch College of General Practitioners' (NHG) guideline on smoking cessation (Chavannes et al., 2007) and the STIMEDIC $®$ guideline (Partnership Stop met Roken, 2009). Both the NHG and STIMEDIC $®$ guideline build on the Minimal Intervention Strategy for general practices (H-MIS). The H-MIS was developed based on the 5 A's strategy (Ask, Advice, Assess, Assist, Arrange) which is part of the clinical practice guideline for treating tobacco use and dependence in the United States (Piasecki et al., 2000) and has proven to be effective in aiding Dutch smokers to quit (Pieterse et al., 2001). Although GPs consider smoking cessation activities as part of their work and value these as highly important in their contribution to health promotion (McAvoy et al., 1999), at the same time they report a lack of time and skills to assist smokers to quit on a large scale (Hoving et al., 2007b, Yarnall et al., 2003). This is demonstrated by the finding that after its implementation less than $30 \%$ of Dutch GPs reported to use the H-MIS and even among these GPs it is unknown to what extent optimal adherence to this guideline was achieved (Kwaliteitsinstituut voor de Gezondheidszorg CBO, 2004).

Previously, it has been suggested that delegating preventive tasks such as the provision of smoking cessation advice to others within the general practice could decrease the barrier of time constraints reported by GPs to provide their smoking patients with extensive smoking cessation support (Hoving et al., 2006a). One possibility would be to shift our focus from the GP to practice nurses, a relatively new profession in the Netherlands. Practice nurses take over certain tasks from the GP, such as consultation hours with chronically ill patients, e.g. patients with diabetes, asthma, or hypertension, and counselling with patients who want to change their lifestyle (Lamkaddem et al., 2004). In the Netherlands, approximately 9.000 GPs are active (Hingstman and Kenens, 2010), of whom at least $62 \%$ o employ a practice nurse (Hingstman and Kenens, 2007). Based on these figures, it can be estimated that at least 5580 practice nurses are currently working in Dutch primary care. As practice nurses relieve GPs by providing care and counselling to these specific patient groups, thereby increasing the overall quality of care in the general practice setting, their additional value within the general practice setting has yet been recognized (Lamkaddem et al., 2004). Furthermore, reasonable evidence exists that nurse-delivered smoking cessation interventions in primary care are effective (Rice and Stead, 2008). Counselling by a practice nurse in combination with free nicotine patches, for instance, resulted in increased rates of point prevalence abstinence and 
continued abstinence (Zwar et al., 2010), as did a nurse-managed minimal-contact smoking cessation intervention for cardiac inpatients (Bolman et al., 2002b). While thus a promising alternative for the delivery of smoking cessation advice by GPs, practice nurses also report reasons such as a lack of time for not implementing smoking cessation interventions in their daily practice (Hall and Marteau, 2007, Nagelhout, 2007, Hall et al., 2005). As the workload of Dutch primary care professionals in general continues to increase (Laurant et al., 2008, van den Berg et al., 2003) this emphasises the importance of developing smoking cessation interventions that do not require an extensive amount of time from the practice nurse.

\section{Computer-tailored smoking cessation interventions}

Computer-tailoring entails the adaptation of intervention materials to the specific characteristics and situation of an individual smoker, using computer software programs (de Vries and Brug, 1999). As opposed to more static tools to provide information and to encourage behavioural change, such as mass media campaigns or brochures, computer-tailored interventions provide individuals only with information that is relevant to them specifically. As a result, this information is more likely to be read, remembered and considered as personally relevant, resulting in a more thorough processing of information (Kreuter et al., 1999, de Vries and Brug, 1999). Computer-tailoring has been found to be effective in changing health behaviour in general (Krebs et al., 2010, Lustria et al., 2009, Noar et al., 2007) and in increasing both short and long term smoking cessation rates (Dijkstra et al., 1998a, Etter and Perneger, 2001b, Lemmens et al., 2008, Te Poel et al., 2009).

As shown in figure 1.2, the computer-tailoring process involves several steps (Dijkstra and De Vries, 1999). First of all, based on the theoretical framework chosen a screening instrument is developed. Most often, this screening instrument consists of a questionnaire with questions about a person's behaviour and presumed behavioural determinants (van Keulen et al., 2008, van Stralen et al., 2008). Secondly, the data gathered using the screening instrument is accumulated into a large data file. A computer software program subsequently matches these data with feedback messages from a feedback library and combines the feedback messages relevant for a particular person into one tailored feedback letter. Computer-tailored interventions are often personalized, i.e. start with a personalized beginning, such as 'Dear Ms. Smit'. A recent study into the working mechanisms of computertailoring showed that the provision of tailored feedback as well as personalization are the most important mechanisms through which computer-tailored interventions work (Dijkstra, 2005). 


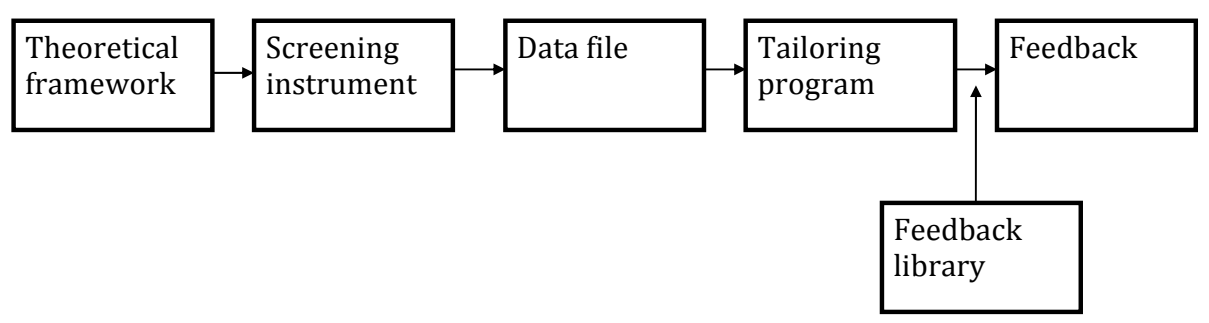

Figure 1.2 The computer-tailoring process.

While previous studies have shown that a single tailored intervention resulted in significantly more quit attempts than no intervention (Dijkstra et al., 1998a, Te Poel et al., 2009, Hoving et al., 2010), the impact of tailored interventions can be even further increased when tailored information is provided on more than one occasion (multiple tailoring) (Borland et al., 2004, Dijkstra et al., 1998c). Within multiple computer-tailored interventions, the computer-tailoring process as described above repeats itself multiple times. This creates the possibility to provide smokers with iterative feedback, which implies that the feedback respondents receive later on during the intervention not only concern the person's present state, but also refer to changes the respondent made compared with earlier measurements. A recent meta-analysis conducted by Krebs and colleagues found support for this idea; they found that dynamically tailored interventions, consisting of multiple assessments and iterative feedback, resulted in greater changes in health behaviour than statically tailored interventions, consisting of multiple assessments and static feedback (Krebs et al., 2010). This suggests that a computer-tailoring approach is warranted that not only provides feedback on multiple moments in time but also provides feedback that is of iterative nature.

The first generation of computer-tailored interventions was mostly paperbased (Dijkstra et al., 1998a, Hoving et al., 2010). Within such interventions, a respondent completed a paper questionnaire and, after completion, returned this questionnaire to the research team by mail. The research team, subsequently, entered the respondent's answers to the questions in the questionnaire into a data file, after which a computer software program generated tailored feedback. This tailored feedback was printed and subsequently sent to the respondent again by mail. During recent years, the Internet has been discovered as an increasingly popular gateway for delivering computer-tailored and smoking cessation interventions (Lustria et al., 2009, Shahab and McEwen, 2009, Te Poel et al., 2009). Using the Internet as a medium for delivering these programmes has clear advantages for both provider and receiver. First, it is highly accessible for smokers both with a low ( $>90 \%$ with Internet access), medium (>98\% with Internet access) and high level of education ( $>99 \%$ with Internet access), as well as for smokers within ( $>98 \%$ with Internet access) and outside ( $>88 \%$ with Internet access) the working population (Centraal Bureau voor de Statistiek, 2011). Secondly, the Internet enables health 
promoters to reach a large audience against minimal costs (Civljak et al., 2010) and a third advantage is that participants can take part at any time that is convenient to them (Griffiths et al., 2006). Fourthly, using the Internet for the delivery of computer-tailored interventions ensures that the behavioural feedback refers to the respondent's current situation and not to his situation during the previous week, as was most often the case with paper-based computer-tailoring. Moreover, smokers might not succeed the first time they try to quit (Hughes et al., 2004) and seeking help online relatively anonymously may prevent them from feelings of failure and embarrassment; negative feelings that are related to a higher temptation to smoke (Rabois and Haaga, 2003) and poorer abstinence outcomes (Leventhal et al., 2008). Two recent reviews showed that smoking cessation interventions delivered through the Internet, such as web-based computer-tailoring, can indeed be effective (Civljak et al., 2010, Shahab and McEwen, 2009). Nevertheless, outside scientific studies an Internet-based computer-tailored smoking cessation programme including multiple, iterative feedback letters was not yet available for the Dutch general public.

\section{The PAS interventions}

Two versions of the PAS intervention were developed. The first version consisted of the Internet-based multiple computer-tailored smoking cessation programme only (MT). As such a programme was not earlier available for the Dutch smoking population the effectiveness of this programme was investigated within a general population sample of smokers. The second version combined the Internet-based multiple computer-tailored smoking cessation programme with tailored counselling by a practice nurse (MTC). As described earlier, there is a need for the development of smoking cessation interventions that do not require an extensive amount of time from primary care staff. Due to the fact that computer-tailored interventions can automatically generate tailored feedback and are increasingly delivered online (Lustria et al., 2009, Shahab and McEwen, 2009) the integration of the Internetbased multiple computer-tailored smoking cessation programme within the general practice setting might be able to limit the burden on health professionals and could potentially be time-, and thus cost, saving. We therefore aimed to test the effectiveness of the combined intervention when integrated in a general practice setting.

In the following paragraphs a brief description of both interventions is provided. For a more extensive description of the development of PAS, I would like to refer the reader to the second chapter of this dissertation.

\section{Internet-based multiple computer-tailored smoking cessation programme}

The Internet-based multiple computer-tailored smoking cessation programme was developed based on previously developed effective single computer-tailored interventions for smoking cessation (Dijkstra et al., 1998a, Hoving et al., 2010, Te Poel et al., 2009). Respondents received a total of four feedback letters. The first tailored feedback letter (4-5 pages) was received at baseline. After completion of the base- 
line questionnaire and receiving most of their tailored feedback, respondents were asked to set a quit date within the upcoming four weeks. Two days after this set quit date, respondents received their second online questionnaire as well as their second feedback letter (1 page). Six weeks and six months after baseline, respondents received their third and fourth questionnaires and tailored feedback letters (both 45 pages). Feedback was tailored to several respondent characteristics: gender, cognitive variables (attitude, social influence and self-efficacy), intention to quit smoking, goal and relapse prevention strategies (preparatory and coping plans), and smoking behaviour. The second tailored feedback letter only provided respondents with relapse prevention strategies as recent studies showed that more than half of ex-smokers relapse within the first week after their quit attempt (Hoving et al., 2006b, Zhou et al., 2009, Hughes et al., 2004), suggesting the need for smoking cessation interventions to provide support especially during smokers' first week of abstinence. Feedback letters were personalized and of iterative nature: the second, third and fourth feedback letters did not only concern the respondent's present state, but also referred to changes respondents had made since previous measurements. Respondents were able to directly read their tailored feedback letters on their computer screen, but feedback letters were sent to the respondent by email too.

\section{Tailored counselling by practice nurses}

After receiving the first tailored feedback, respondents in the MTC group were prompted to schedule a counselling meeting with their practice nurse within 6 to 8 weeks. They received this counselling session instead of the third tailored feedback letter respondents in the MT group received at six-week follow-up. To assist practice nurses in guiding these counselling sessions, they were provided with a counselling protocol. This protocol was developed based on existing counselling protocols for smoking cessation (Chavannes et al., 2007, Pieterse et al., 2001) and on a needs assessment conducted among practice nurses. The protocol consisted of three chapters guiding counselling sessions with three different types of respondents: smokers who had quit successfully, smokers who quit but relapsed, and smokers who had not tried to quit yet. The content of the counselling sessions was developed to be as similar as possible to the content of the computer-tailored feedback letters and was also tailored to the respondent characteristics mentioned in the previous section. After six months, practice nurses were instructed to call participating patients to ask them about their progress towards permanent cessation and, if needed, to provide them with additional telephone counselling.

\section{EVALUATION}

When effective, smoking cessation interventions have the potential to impact on population health by reducing premature death rates and increasing the number of years in good health (USDHHS, 2004, WHO, 2008). To evaluate interventions for 
their effectiveness, the strongest and most appropriate design, widely perceived as the 'golden standard', is the randomized controlled trial (RCT) (Campbell et al., 2000, Flay, 1986, Flay et al., 2005). Within an RCT, the effect of the intervention is tested against a control group, i.e. a group of participants not receiving the intervention. This neutralizes the influence of naturally occurring changes in smoking behaviour, thereby providing a more accurate representation of the intervention's effectiveness (Campbell et al., 2000, Flay, 1986, Flay et al., 2005). While many RCTs have been conducted investigating the clinical effectiveness of behavioural smoking cessation interventions, similar studies investigating the cost-effectiveness of such interventions are limited in number. Therefore, the PAS interventions have been evaluated both with regard to their effectiveness and regarding their costeffectiveness.

\section{Effectiveness evaluation}

To evaluate both versions of PAS with regard to their effectiveness in achieving the target behaviour, i.e. smoking abstinence, two RCTs were conducted. The first RCT was conducted to test the effect of MT within a general population sample of smokers. Within this RCT, the control group did not receive any intervention. The second RCT was conducted within a general practice setting and tested the effect of both MTC and MT among a sample of smokers recruited via Dutch practice nurses. To imitate a natural situation, respondents in the control group of this RCT received smoking cessation guidance according to standard practice. In the Netherlands, standard practice varies from a brief intervention consisting of a single stop smoking advice to more intensive interventions consisting of four or more consultations (Chavannes et al., 2007, Partnership Stop met Roken, 2009). In both studies, both the intervention and control group were free to use smoking cessation aids other than those provided during the entire study period.

Previously, several factors have been found to moderate the effects of smoking cessation interventions on smoking cessation outcomes. Such factors include the level of nicotine dependence (Fucito et al., 2010, Willemsen et al., 1998), the presence of a tobacco-related illness, the presence of non-smoking children in the household and frequent alcohol consumption (Strecher et al., 2006). Following recent recommendations to determine participant characteristics associated with effectiveness (Noar et al., 2007), in both RCTs we have therefore explored whether intervention effects were different for specific subgroups of smokers, in addition to investigating main intervention effects.

Effectiveness of both interventions was operationalized in terms of significant differences between the intervention and control group with regard to three measures of smoking abstinence. In line with the Russell standard (West et al., 2005), smoking abstinence was assessed using three different measures: prolonged abstinence (i.e. abstinence from smoking since the previous measurement), seven-day point prevalence abstinence (i.e. abstinence from smoking during the past seven 
days) and 24-hour point prevalence abstinence (i.e. abstinence from smoking during the last 24 hours).

With regard to the effects of the two versions of the PAS intervention on these measures of smoking abstinence, the present dissertation aimed to answer the following research questions:

1. Does the Internet-based multiple tailored smoking cessation programme have a significant effect on smoking abstinence when compared to a no intervention control group in a general population sample of smokers?

2. Do both PAS interventions have a significant effect on smoking abstinence when compared to care as usual in a sample of smokers recruited in a general practice setting?

3. Do the effects of the PAS interventions differ for subgroups of smokers participating in these two studies?

\section{Cost-effectiveness evaluation}

An economic evaluation study aims to determine the costs and effects associated with an intervention and to compare these with other interventions and/or current practice. Results from economic evaluation studies are of great value in making evidence-based decisions on the widespread implementation or dissemination of interventions (Evers et al., 2010). Especially when considering the current political climate in the Netherlands with large budget cuts across all governmental departments and only limited funds available for implementing health promotion programmes (Schippers, 2011), information about the relative cost-effectiveness of interventions aimed at health promotion or disease prevention is needed to inform decision-making processes. In economic evaluation studies effects are frequently operationalized in terms of quality of life (Evers et al., 2010). Governments often want to compare the cost-effectiveness of interventions targeting different diseases and using quality of life as the main measure of effect facilitates this comparison. The ratio between an intervention's additional costs and additional effects in terms of quality of life can be referred to as the intervention's cost-utility. However, to compare the cost-effectiveness of different smoking cessation interventions more specifically, lifestyle-specific effect measures, e.g. smoking abstinence, might be more informative. In the present dissertation, we refer to the ratio between an intervention's additional costs on the one side and additional effects in terms of smoking abstinence on the other as the intervention's cost-effectiveness.

Previously, a cost-effectiveness analysis of tailored print communication aimed at increasing physical activity levels and fruit and vegetable consumption (van Keulen et al., 2010), showed that with a willingness to pay of $€ 160$ per additional guideline met and €2,851 for each quality adjusted life year (QALY) gained, 
tailored print communication had a high probability of being more cost-effective than telephone motivational interviewing, a combination of both interventions or no intervention. Furthermore, a web-based computer-tailoring programme added to the provision of fruit and vegetables and a classroom curriculum has been shown to be cost-effective in increasing fruit and vegetable consumption amongst schoolchildren, both when compared with the provision of fruit and vegetables only and in comparison to no intervention (te Velde et al., 2011). Since there was a need for research to economically evaluate eHealth interventions (Ahern et al., 2006), some studies have been conducted towards the cost-effectiveness and cost-utility of Internet-based interventions aimed at reducing alcohol use (Smit et al., 2011) and depressive symptoms (Warmerdam et al., 2010, Gerhards et al., 2010). So far, however, no economic evaluation studies have been conducted concerning the costeffectiveness and cost-utility of an Internet-based computer-tailored smoking cessation intervention such as PAS. Therefore, this dissertation aims to answer the following research question:

1. What is the cost-effectiveness and cost-utility of both PAS interventions when implemented in a general practice setting compared to care as usual and to each other?

\section{DISSEMINATION}

The impact of an intervention on public health can be defined as Effect x Implementation x Reach: the intervention's public health impact depends not only on its effectiveness, but also on its implementation by intermediate groups and on its reach amongst the target group (Glasgow et al., 2003).

\section{Implementation}

Previous research has shown that despite the existence of effective smoking cessation interventions (Fiore, 2000, Lancaster and Stead, 2005b, Pieterse et al., 2001, Van den Bruel et al., 2004, van Weel et al., 2005), the impact of these interventions on public health is often suboptimal as many primary care professionals do not adopt these interventions or do not implement them as intended (Chavannes et al., 2007, Eccles et al., 2007, Glasgow et al., 2003, Segaar et al., 2007). To understand the dissemination process of health behaviour change interventions, both the Diffusion of Innovations theory (Rogers, 1995) and the ICM (De Vries et al., 2003) have been used frequently as a theoretical framework (Bolman et al., 2002a, Helmink et al., 2011, Hoving et al., 2007b, Lee, 2004, Pronk et al., 2001, Puffer and Rashidian, 2004, Rogers, 2002, Segaar et al., 2006, van der Weide and Smits, 2004).

The Diffusion of Innovations theory (Rogers, 1995) suggests that the dissemination process consists of three main phases, of which adoption and implementa- 
tion are the first two. According to this theory, adoption refers to the phase in which a decision-maker learns of the existence of an intervention (knowledge) and, subsequently, forms a favourable or unfavourable attitude towards this intervention (persuasion). This decision-makers' attitude is supposed to be the most important determinant of the decision to either adopt or reject the intervention (decision) and is considered to be based on the perception of five intervention characteristics: relative advantage, compatibility, complexity, trialability and observability (Rogers, 1995). These ideas have found support in several studies investigating the factors associated with the adoption of novel interventions; in several studies positive beliefs about smoking cessation interventions have been identified as an important reason for adopting smoking cessation interventions among general practice staff, while negative beliefs were reported as reasons for not adopting (Chavannes et al., 2007, Coleman et al., 2001, Hall and Marteau, 2007, Hall et al., 2005, Hoving et al., 2006a). The Diffusion of Innovations theory describes the implementation phase as the actual implementation of an intervention once it has been adopted. In previous studies addressing the determinants of the implementation of health behaviour change interventions, health professionals' clinical behaviour could be largely explained by their intention to implement (Eccles et al., 2006). Furthermore, a positive attitude, positive social norms and a higher self-efficacy were found to be positively associated with the intention to implement smoking cessation programmes in general practice (Hoving et al., 2007b, Puffer and Rashidian, 2004, Helmink et al., 2011) as well as in other healthcare settings (Bolman et al., 2002a, Segaar et al., 2007). Furthermore, organization-related factors like structural and practical barriers (e.g. a lack of time) were previously identified as negatively influencing the implementation of smoking cessation interventions by general practice staff (Hall and Marteau, 2007, Nagelhout, 2007).

Despite these previous research findings, the gap between evidence and practice remains (Eccles et al., 2007, Glasgow et al., 2003, Stead et al., 2009). This emphasizes the persistent need to study the factors associated with the adoption and implementation of novel smoking cessation interventions. Besides focussing on the well-investigated cognitive factors described above, however, studies should aim to identify other factors influencing the dissemination process. One such factor is the health professional's satisfaction with current practice. While past research has reported much about patients' satisfaction (i.e. compliance with recommendations from the health professional rises when the patient is satisfied with the consultation (Shum et al., 2000, van Son et al., 2004, Derckx, 2006), there is a shortage of research about the potential influence of practice nurses' satisfaction with the current smoking cessation activities that they are involved in. Supported by other studies investigating the determinants of adopting a smoking cessation intervention by health care professionals (Bolman et al., 2002a, Hall et al., 2005), it can be expected that practice nurses' dissatisfaction with current smoking cessation activities is related to a more positive attitude towards the new intervention and a higher intention to adopt this intervention. Another factor that warrants further investigation is 
the practice nurses involvement with the intervention. A previous study showed that involvement in a trial testing the effectiveness of a smoking cessation intervention was an important determinant of GPs' and practice nurses' intention to implement this intervention (Hoving et al., 2007b). It would therefore be relevant to investigate whether practice nurses who recruited more patients to participate in PAS, and who could thus be considered more involved, would report a higher intention to implement the intervention than those who recruited fewer patients and could be considered less involved with the intervention. To inform the development of intervention strategies aimed at increasing adoption and implementation rates of effective smoking cessation interventions, this dissertation aims to answer the following research questions regarding the dissemination process of PAS:

1. Do cognitive factors and satisfaction with current practice determine practice nurses' intention to adopt PAS?

2. Do cognitive factors and involvement in the trial determine practice nurses' intention to implement PAS?

\section{Reach}

For an intervention to have an impact on public health, it is not only required that practice nurses adopt and implement the intervention, but also that the intervention reaches smokers eligible to take part in the programme. However, the recruitment of smokers for participation in smoking cessation trials in a primary care setting is challenging and participation rates are often low (Gilbert et al., 2012). Previously, both active and passive recruitment methods employed in primary care have been shown to be relatively successful in reaching smokers, including smokers with a low socio-economic status, for participation in a smoking cessation intervention (Hoving et al., 2007a). Recruitment using relatively more proactive methods has, however, been suggested to be most successful (Gilbert et al., 2012, Hoving et al., 2007a).

As the Internet is becoming an increasingly popular medium for delivering health behaviour change programmes, it is important to investigate the effectiveness of recruitment strategies other than those employable in a health care setting. Although the Internet is able to reach large amounts of people (Griffiths et al., 2006), it is not yet clear whether all segments of the smoking population, such as smokers with a low socio-economic status who are often hard to reach, can be successfully reached through this medium (Brouwer et al., 2010, Lorence and Park, 2008, van Dijk, 2003).

To recruit smokers for participation in the two RCTs conducted to investigate the effectiveness of the PAS interventions, two different recruitment strategies were used. For participation in the first RCT, practice nurses working in Dutch gen- 
eral practices both passively and actively recruited smokers from their patient population. For participation in the second RCT, recruitment of smokers took place via mass media channels, such as regional newspapers, advertisements, an online social network website (Hyves) and online smoking cessation forums. This allowed us to investigate whether recruitment via general practices and recruitment via (online) mass media resulted in different samples of smokers willing to participate in an Internet-based multiple computer-tailored smoking cessation programme, with regard to size and demographic characteristics. In addition, it enabled us to determine whether the choice for a particular recruitment strategy had an influence on participants' retention rates. This is especially relevant since web-based interventions are known to often suffer from high levels of attrition (Blankers et al., 2010, Eysenbach, 2005, McKay et al., 2008, Shahab and McEwen, 2009, Webb, 2009, West et al., 2005), which negatively influences these interventions' potential effects on behaviour (Krebs et al., 2010). Therefore, the present dissertation aims to answer the following research questions regarding the reach of PAS amongst the target population and its subsequent effect on retention and success rates:

1. Do recruitment via general practices and recruitment via (online) mass media result in a significantly different number and type of Dutch adult smokers participating in an Internet-based multiple tailored smoking cessation intervention?

2. Do significant differences exist in retention rates between these two differently recruited samples of smokers?

3. Do significant differences exist in quitting behaviour between these two differently recruited samples of smokers?

\section{MOTIVATION}

A positive motivation to quit is considered a necessary prerequisite for smokers to actually quit smoking (Hyland et al., 2006, Norman et al., 1999, Vangeli et al., 2011). As a consequence, several previous studies investigating the effectiveness of smoking cessation interventions have only considered smokers eligible to participate when they were motivated to quit smoking within a relatively short period of time (e.g. Hoving, 2007, Te Poel et al., 2009). Nonetheless, not all smokers who participated in these interventions undertook an attempt to quit smoking. This might be explained by perceived barriers to quit smoking and to maintain smoking abstinence, contributing to the intention-behaviour gap described by several theorists (e.g. Armitage and Conner, 2001, De Vries et al., 2003, Godin and Kok, 1996). Yet, another explanation could be that frequently used measures of motivation represent an overestimation of the percentage of smokers genuinely motivated to quit. This latter possibility emphasizes that to inform the development of future smoking 
cessation intervention strategies and to ultimately be able to increase smoking abstinence rates, a further understanding of smokers' motivation to quit is warranted. Currently, multiple theoretical perspectives on motivation exist. Those most often used in contemporary research are described in the following; 1) motivation as a continuous measure of intention; 2) extrinsic versus intrinsic motivation; and 3) motivation subdivided into stages based on the time period within the person intends to change.

\section{Motivation as intention}

The ICM (De Vries et al., 2003) as well as other socio-cognitive theories (e.g. the TPB (Ajzen, 1985)), have incorporated intention as the most proximal predictor of behaviour. To date, a great amount of evidence has been gathered for intention being the most important predictor of behaviour change in general (Armitage and Conner, 2001, Godin and Kok, 1996) and of smoking cessation behaviour in particular (Armitage and Conner, 2001, Hyland et al., 2006, Norman et al., 1999). However, a significant part of the variation in (non-)smoking behaviour remains unexplained by intention (Armitage and Conner, 2001, Godin and Kok, 1996). For example, while about $75 \%$ of the Dutch adult smokers expresses an intention to ever quit smoking, only a minority of them actually make a quit attempt and only few do so successfully (STIVORO, 2010). A potential reason for this gap between intention and behaviour might be that the percentage of smokers said to express an intention to quit smoking represents an overestimation of the percentage of smokers genuinely motivated to quit. As several countries, such as Great Britain, Ireland and the Netherlands, have adopted a national smoking ban in public places, public norms are shifting towards non-smoking (Orbell et al., 2009). This may yield smokers to believe that they ought to quit smoking, even though they might not want to, resulting in an overestimation of the percentage of smokers motivated to quit. As this might lead to a misunderstanding of the effectiveness of smoking cessation interventions, it may be relevant to make a distinction between different types of motivation, including intention.

\section{Extrinsic and intrinsic motivation}

An example of a theory of motivation that distinguishes between different types of motivation is Self-Determination Theory (SDT) (Ryan and Deci, 2000). This theory makes a distinction between relatively controlled or extrinsic types of motivation and more autonomous or intrinsic motivation. According to SDT, intrinsically motivated behaviours are considered to be driven by the desire to achieve rewards that are internal to the person (e.g. one's health), while extrinsically motivated behaviours are thought to be in response to external rewards, punishments or social expectations (e.g. money or social pressure) (Deci and Ryan, 1988). Previous research has found support for this distinction by showing that smokers' reasons to quit could indeed be subdivided into two types of intrinsic reasons, i.e. concerns about 
health and a desire for self-control, and two types of extrinsic reasons, i.e. immediate reinforcement and social influence (Curry et al., 1990, Curry et al., 1997). Both extrinsic and intrinsic motivation have been found to be able to initiate attempts to quit smoking (Ryan and Deci, 2000). When it concerns actual smoking cessation and the maintenance of non-smoking, however, smokers with higher levels of intrinsic relative to extrinsic motivation have been shown to be more likely to be successful (Curry et al., 1990, Williams et al., 2006b).

The distinction between extrinsic and intrinsic motivation is in congruence with the suggestion from SCT (Bandura, 1991) that behaviour can be influenced both by intrinsic and by extrinsic anticipated incentives or disincentives and with the terminology used in the ICM (De Vries et al., 2003), describing that behavioural pros and cons can be perceived as intrinsic or extrinsic to the person. Another view similar to SDT's perspective on motivation can be derived from PRIME theory of motivation (West and Hardy, 2006). This theory distinguishes 'motives/desires' (feelings of want and need relating to an imagined future) from 'evaluations' (beliefs about what is good or bad) and 'plans' (intentions regarding future actions) (West, 2009, West and Hardy, 2006). The distinction between motives/desires and evaluations is in congruence with SDT (Deci and Ryan, 2008); while a desire to quit smoking might be considered a form of intrinsic motivation, beliefs about duty to quit can be considered extrinsic types of motivation. Whereas SDT does not specifically address how intention is associated with either intrinsic or extrinsic motivation, PRIME theory predicts that intention would only influence quitting behaviour if accompanied by a desire, or intrinsic motivation, to quit smoking (West, 2009, West and Hardy, 2006). Similarly, this theory hypothesizes that duty is not an independent predictor of quitting behaviour, but would influence quitting behaviour only in combination with a desire to quit (West, 2009, West and Hardy, 2006). These hypotheses are in contradiction with earlier suggestions that intention is the most important predictor of smoking cessation (Armitage and Conner, 2001, Hyland et al., 2006, Norman et al., 1999) and with the suggestion from SDT that both extrinsic and intrinsic motivation are able to initiate behaviour change (Ryan and Deci, 2000). Disentangling the roles played by these different types of motivation (i.e. desire, duty and intention) in predicting smoking cessation behaviour would be useful in identifying optimal markers of motivation and in ultimately designing more effective interventions to promote quitting behaviour. To date, there appears to be no research differentiating these three aspects of motivation. This dissertation aims to contribute to filling this gap in the literature by answering the following research questions: 
1. What is the prevalence of desire, duty and intention to quit smoking?

2. Do desire, duty and intention independently predict attempts to quit smoking?

3. What is the predictive value of each potential combination of these three types of motivation?

\section{Stages of motivation}

As described earlier, a smoker's motivation to quit smoking can also be measured based on the time period within the person intends to change his or her behaviour using a so-called stage algorithm. Examples of stage algorithms are the stages of change algorithm used in the TTM (Prochaska and DiClemente, 1983, Prochaska et al., 1997) and the ICM (De Vries et al., 2003), or the stages of behavioural change as suggested by the Health Action Process Approach (HAPA) (Schwarzer, 2004) or the Precaution Adoption Process Model (PAPM) (Weinstein and Sandman, 2002). In general, stage theories of health behaviour change assume that people within the same stage face the same barriers and facilitators to change, while people in different stages are confronted with different barriers and facilitators (Weinstein et al., 1998). As a consequence, if progress through the stages is to occur health educators should adapt their messages to the determinants of behaviour change relevant to the stage an individual is in (Norman et al., 2000, Weinstein et al., 1998). Previous research has found support for the idea that people in different stages or motivational phases may profit from different types of information (de Vries and Mudde, 1998, De Vries et al., 1998, Dijkstra et al., 2006). Besides, a number of stage-based smoking cessation programmes have previously been developed and proven effective (Dijkstra et al., 1998b, Prochaska et al., 2008, Erol and Erdogan, 2008, Meyer et al., 2008, Dijkstra et al., 2006).

Despite the support in favour of a stage-based approach, several theorists have questioned the viability of the stages of change as the underlying conceptualization of health promotion interventions (Heather, 1991, Sutton, 2001, Littel and Girvin, 2002, de Vet et al., 2007, West, 2005, Bandura, 1998). Main criticism on staging algorithms in general is that the differentiation between stages is based on arbitrary time periods (Sutton, 2001, West, 2005, Bandura, 1998). For instance, a smoker is in the preparation stage if he is planning to stop smoking within the next 30 days, while this same smoker would be in the contemplation stage when reporting to plan to quit within 31 days' time (Sutton, 2001). Doubt exist in particular concerning the validity of the contemplation stage of change, consisting of those intending to quit within six months but not within one month. This stage has been suggested to be rather unstable (Velicer et al., 1999) and heterogeneous and to encompass a mixture of smokers who would be classified as moderate to high in 
motivation to quit by alternative measures of motivation (Herzog, 2008). As also suggested by other theorists, this might indicate that the contemplation stage of change may not be a genuine stage (West, 2005, Herzog, 2008, Sutton, 2001, Bandura, 1998) and is in fact not different from other stages of change, or that this stage is too comprehensive.

The idea that subtypes exist within the contemplation stage has been explored in three earlier studies (Anatchkova et al., 2005, Norman et al., 2000, Velicer et al., 1995). In all these studies, four subtypes within contemplation were identified: the Classic contemplator (with above average scores on both the pros and cons of smoking, resulting in a cognitive conflict, and high scores on the perceived temptations to smoke), the Early contemplator (with a profile corresponding to precontemplation; scoring higher on the pros than on the cons of smoking and perceiving average temptations to smoke), the Progressing contemplator (with a profile corresponding to preparation; with the cons outweighing the pros of smoking, and still perceiving average temptations to smoke) and a subtype demonstrating a lack of concern regarding both pros and cons and situational temptations, labelled Disengaged (scoring slightly below or above average on all scales). However, these previous studies clustered respondents based on the pros and cons of smoking and perceived temptations to smoke. According to Ajzen's compatibility principle (Ajzen, 1988), a more accurate cluster solution could be expected to be identified when using clustering variables that are measured in a similar manner as the target behaviour (i.e. quitting smoking). Therefore, to further explore the idea that subtypes exist within the contemplation stage of change, the present dissertation aims to answer the following research questions:

1. Can subtypes of smokers in contemplation be identified based on quittingrelated variables (i.e. the pros and cons of quitting and self-efficacy to quit)?

2. Do subtypes of smokers differ with regard to other baseline variables?

3. Are subtypes predictive of smoking abstinence and stage transition six months after baseline?

\section{AIMS OF THE DISSERTATION}

The present dissertation aims to answer eight major research questions, covering the more specific research questions described in the preceding sections. These eight research questions can be clustered around three major subjects: the evaluation of PAS, the dissemination of PAS and the construct of motivation. 


\section{Evaluation}

The first three research questions to be answered in the present dissertation concern the evaluation of the two PAS interventions regarding their effectiveness, costeffectiveness and cost-utility:

1. What is the effect of an Internet-based multiple computer-tailored smoking cessation programme on smoking abstinence when compared to a no intervention control group among a general population sample of smokers?

2. What is the effect of an Internet-based multiple computer-tailored smoking cessation programme combined with and without tailored counselling by a practice nurse on smoking abstinence when compared to care as usual among smokers recruited in a primary care setting?

3. What is the cost-effectiveness and cost-utility of an Internet-based multiple computer-tailored smoking cessation programme combined with and without tailored counselling by a practice nurse among smokers recruited in a primary care setting compared to care as usual and to each other?

\section{Dissemination}

The subsequent three research questions aim to contribute to the literature by studying the dissemination of PAS, thereby focussing on the adoption and implementation of the interventions as well as on the interventions' reach amongst the target population:

4. What are the determinants of practice nurses' intention to adopt a new smoking cessation intervention?

5. What are the determinants of practice nurses' intention to implement a new smoking cessation intervention?

6. What is the influence of recruitment strategy on the reach and effect of an Internet-based multiple tailored smoking cessation intervention amongst Dutch adult smokers?

\section{Motivation}

The last two research questions both aim to contribute to an increased understanding of smokers' motivation to quit:

7. What is the role of desire, duty and intention to quit smoking in predicting quit attempts?

8. Do subtypes exist within the contemplation stage of change?

\section{OUTLINE OF THE DISSERTATION}

Before answering the research questions related to the evaluation of PAS, chapter 2 provides an extensive description of the development of PAS. In chapter 5 the results are described from the RCT which investigated the effectiveness of the Internet-based multiple computer-tailored smoking cessation programme among a general population sample of Dutch adult smokers. Chapter 6 presents the results from 
the RCT testing the effectiveness of the two PAS interventions when compared with care as usual in the general practice setting. The results from the trial-based economic evaluation that was conducted to investigate the cost-effectiveness and costutility of both PAS interventions when implemented in a primary care setting are described in chapter 7 .

The research questions with regard to the dissemination of PAS will be answered in chapter 3, 4 and 8. Chapter 3 describes a cross-sectional study among Dutch practice nurses and nurse practitioners that aimed to identify factors associated with their intention to adopt the new smoking cessation intervention PAS. Chapter 8 describes the results from a cross-sectional study that investigated the determinants of practice nurses' intention to implement PAS after a trial period. The influence of recruitment strategy on the reach and (short-term) effect of the Internet-based multiple tailored smoking cessation programme was investigated in the study described in chapter 4.

Chapter 9 and 10 aim to answer the research questions formulated with regard to the construct of motivation. Chapter 9 describes the results of a longitudinal study which aimed to assess the predictive value of desire, duty and intention to quit, three different aspects of motivation, in predicting attempts to quit smoking among a nationally representative sample of British smokers. Chapter 10 presents the results of a study that aimed to investigate whether subtypes exist within the contemplation stage of change and to compare the subtypes identified with regard to their smoking status and stage of change after six months.

In the final chapter of this dissertation, chapter 11, the results presented in the different chapters are integrated and discussed and their implications for research and practice are described. 



\section{CHAPTER 2}

The PAS study:

A randomized controlled trial evaluating the effectiveness of a web-based multiple computertailored smoking cessation programme and tailored counselling by practice nurses

Published as: Smit, E.S., de Vries, H., Hoving, C. (2010). The PAS study: A Randomized Controlled Trial evaluating the effectiveness of a web-based multiple tailored smoking cessation programme and tailored counselling by practice nurses, Contemporary Clinical Trials, 31, 251-258. 


\section{ABSTRACT}

Background PAS (Personal Advice in Stopping smoking) combines two of the most effective smoking cessation interventions: multiple computer tailoring and tailored counselling by a practice nurse in the general practice.

Methods/design Since May 2009, practice nurses are recruiting smoking patients. Each practice nurse is asked to recruit 15 adult smokers who are willing to quit within 6 months and have access to the Internet. Smokers can sign up for PAS through the PAS website and are then randomized into one of three groups receiving multiple tailoring and counselling (MTC), multiple tailoring (MT) or usual care (UC), respectively. All groups receive questionnaires at baseline, 2 days after a set quit date and at 6 weeks, 6 months and 12 months follow-up. The MT group receives tailored, iterative feedback letters at the first four measurements points. At 12 months follow-up biochemical validation will take place amongst respondents reporting to have quit. The three groups will be compared with regard to quit attempt rate, point prevalence abstinence and continued abstinence by means of logistic multilevel regression analyses. Linear multilevel regression analyses will be used to compare the three groups regarding smoking related beliefs.

Discussion The present paper provides an extensive description of the development of PAS and of the design of the study towards its effectiveness. This might provide insight into PAS' potentially effective working mechanisms. The results concerning effectiveness may contribute to knowledge about the effectiveness of smoking cessation interventions aimed at smoking adults.

Trial registration Dutch Trial Register NTR1351 


\section{BACKGROUND}

The smoking of tobacco is the most preventable cause of illness and premature death in the world (USDHHS, 2004, WHO, 2008). To aid smokers to quit successfully, effective smoking cessation interventions are essential. Computer tailored programmes have shown their efficacy (Borland et al., 2004, Lancaster and Stead, 2005c, Noar et al., 2007): a single tailored intervention resulted in significantly more quit attempts compared to no intervention (Dijkstra et al., 1998a, Hoving, 2007) and the impact of tailored interventions even increased when providing tailored information on more than one occasion (multiple tailoring) (Brug et al., 1998, Dijkstra et al., 1998c). But, outside scientific studies, multiple tailored smoking cessation feedback is not yet available to the Dutch general public. A brief smoking cessation advice from a general practitioner (GP) also results in a small though significant effect on quit rates (Lancaster and Stead, 2005a, Stead et al., 2008). GPs consider smoking cessation activities as part of their work and value these as most important in health promotion (McAvoy et al., 1999). However, they also report a lack of time and skills as barriers in assisting smokers to quit on a larger scale (Hoving et al., 2007b, Yarnall et al., 2003). If GPs can delegate preventive tasks to others within the general practice, time constraints could diminish and the adoption of smoking cessation advice will be more likely. One possibility would be to shift our focus from GPs to practice nurses, a relatively new profession in the Netherlands. A practice nurse takes over certain tasks of the GP, such as consultation hours with patients having chronic diseases, e.g. diabetes, asthma, or hypertension. The additional value of practice nurses has been recognized, as they increase the quality of care and relieve GPs by providing care and counselling to these specific patient groups (Lamkaddem et al., 2004). In 2007, 62\% of all Dutch general practices employed a practice nurse (Hingstman and Kenens, 2007). Although computer tailoring and counselling are two of the few effective smoking cessation interventions developed so far (Lancaster and Stead, 2005c), stand-alone interventions are known mostly to have only limited success (Lancaster et al., 2000). Within PAS (Personal Advice in Stopping smoking), multiple computer tailoring and a counselling session with a practice nurse are combined, as the combination of two effective stand-alone interventions can be expected to achieve higher abstinence rates than either of the two alone. Supporting this approach, earlier studies showed that combining a single tailored intervention with counselling has an additional effect compared with tailoring alone, when aiming at smoking cessation (Curry et al., 1995, Sutton and Gilbert, 2007). This paper aims to thoroughly describe the PAS programme, its development and the intervention components it consists of, in order to facilitate the discovery of its effective working mechanisms and to make replication possible. 


\section{METHODS/DESIGN}

PAS was approved by the Medical Ethics Committee of Maastricht University and the University Hospital Maastricht (MEC 08-3-037; NL22692.068.08), and is registered with the Dutch Trial Register (NTR1351). The approval covers all participating general practices in the study.

\section{Recruitment of practice nurses}

From January until March 2009, practice nurses working in general practices in the Netherlands were invited to fill out a short web-based questionnaire concerning the adoption of PAS. They were encouraged to discuss the adoption of PAS with GP(s) and practice nurses they were working with before filling out this questionnaire. Besides aiding in recruitment, this questionnaire also served as a means to assess characteristics of adoptive and non-adoptive practice nurses. Practice nurses who reported to be willing to adopt the intervention or who required more information to make a decision were contacted by telephone in order to be recruited for participation in the effectiveness study. In the first half of 2009, recruited practice nurses were visited by a member of the PAS research team. This visit aimed to explain both study procedures and the PAS counselling protocol and to secure a working relationship with each practice nurse.

\section{Recruitment of smoking respondents}

From May 2009 on, practice nurses started recruiting smoking respondents from their practices. Following procedures used in previous experiments (Prochaska et al., 2005, Yarnall et al., 2003), a recruitment period of 6 months is used for each practice nurse to collect smoking participants from their general practice. Each practice nurse is asked to recruit 15 participants for the study (five for each of the study arms). During the PAS research team's visit, practice nurses were instructed on the information to be given to each potential smoking participant before referring them to the PAS website. In addition, a Frequently Asked Questions (FAQ) section is included in the PAS counselling protocol, providing answers to numerous possible questions patients might ask their practice nurse about the study. To further assist practice nurses in recruiting enough patients, several recruitment materials are made available (e.g. desk displays and PAS business cards).

\section{Inclusion and exclusion criteria for smokers}

Respondents can be included in the study when they currently smoke, are motivated to quit within 6 months, are 18 years or older and are sufficiently proficient in Dutch. Moreover, they have to have access to the Internet. There are no explicit exclusion criteria. 


\section{Randomization}

Interested smokers are directed by their practice nurse to the PAS website (www.persoonlijkstopadvies.nl), where they can sign up for the study with their own username and password. No one but the PAS research team is able to retrieve these passwords. Subsequently, after signing the online informed consent form, participants will be randomly allocated to either the control condition or one of the two experimental conditions. Randomization takes place at respondent level by means of a computer software randomization device. Blinding of respondents and practice nurses is not possible due to recruitment procedures and the requirement that respondents take notice of whether or not they are receiving an intervention.

\section{Study design}

Participants are randomly allocated to either the control condition or one of two experimental conditions (Figure 2.1).

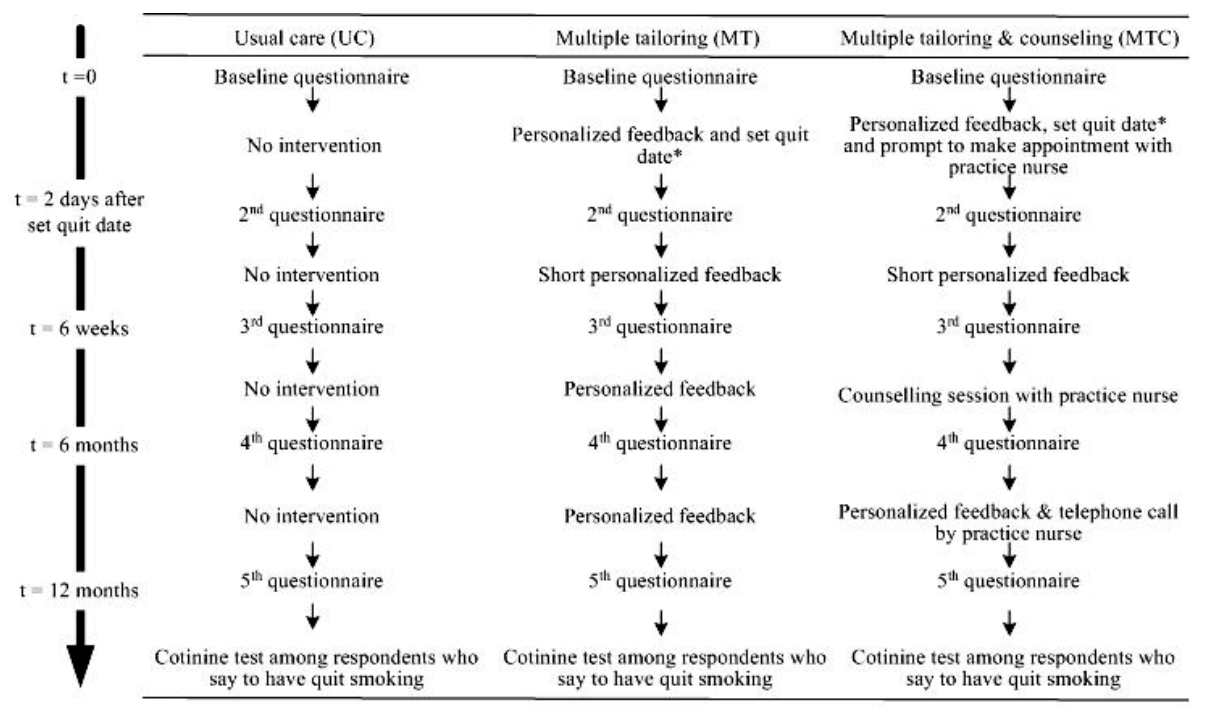

Figure 2.1 Flowchart of the randomized controlled trial testing the effectiveness of PAS: multiple tailoring and counselling by practice nurse.

1. Control group (usual care; UC): Respondents receive care as usual from their general practice. They are thus able to receive any method that is usually used in their general practice to aid patients to quit smoking.

2. Multiple tailoring (MT): Respondents receive four personalized feedback letters at different time points.

3. Multiple tailoring and counselling (MTC): Respondents receive three tailored feedback letters, like the MT group, but one letter (the third) is replaced by a counselling session with a practice nurse. Also, the last tailored feedback letter 
(at 6 months follow-up) is accompanied by a telephone call from the practice nurse involved.

\section{Questionnaires}

In all three study groups, respondents are prompted by e-mail to fill in an online questionnaire at five points in time: at baseline, 2 days after the quit date each respondent sets at baseline, and then at 6 weeks, 6 months and 12 months after filling out the baseline questionnaire. One reminder is sent by e-mail each time that, one week after receiving the invitation, a respondent has still not filled out the particular questionnaire he or she was invited to complete. Questionnaires are based on the I-Change model (De Vries et al., 2003) and have been tested experimentally among Dutch smoking adults in previous studies (Dijkstra et al., 1996, Dijkstra et al., 1998a, Hoving et al., 2007a). Each questionnaire (except the questionnaire two days after the set quit date) consists of questions concerning smoking behaviour, addiction level, attitude, self-efficacy, social influence, action and coping planning and intention to quit smoking and/or to maintain non-smoking (De Vries et al., 2003). At baseline, respondents are asked to plan a quit date within the upcoming 4 weeks. The questionnaire two days after this quit date only consists of questions concerning smoking behaviour and implemented action and coping plans, as we want to minimize the burden of filling out a questionnaire by smokers who have recently quit. For smokers who have quit at follow-up, measurements, questions and feedback about action plans are skipped, as preparatory plans become redundant once the respondent has actually quit. For these respondents, emphasis is laid on coping plans.

\section{Web-based multiple tailored smoking cessation advice}

With tailored health communication, the content of the health message is adapted to the specific characteristics of a particular individual with a view to attracting and keeping the individual's attention (de Vries and Brug, 1999, Kreuter et al., 1999). Baseline computerized feedback messages of the present tailoring programme are based on a previously tested effective theory based single computer tailored intervention (Dijkstra et al., 1998a). The feedback respondents will receive later on during the programme is created by the PAS research team. Feedback letters are personalized and based upon the answers provided by respondents in the questionnaires, as personalization and feedback are both shown to be working mechanisms of tailoring (Dijkstra, 2005, Dijkstra, 2008). Messages are tailored to several respondent characteristics, based on the I-Change model (De Vries et al., 2003): gender, cognitive variables (attitude, social influence and self-efficacy), intention to quit smoking, goal and relapse prevention strategies (action and coping plans), and smoking behaviour. Feedback is item based in order to provide more detailed feedback and to improve personalization of the feedback messages. Moreover, feedback will be iterative, in that the second, third and fourth feedback letters not only con- 
cern the respondent's present state, but also refer to changes (in behaviour, but also in beliefs) made by the respondent compared with earlier measurements. Questions are alternated with feedback in order to improve retention rates. Feedback messages ultimately form a complete feedback letter that is presented to respondents after completion of the questionnaire. All feedback letters, except the one two days after the set quit date, are 4-5 pages in length and consist of seven components:

1. Introduction, including specific behavioural feedback and feedback on the respondent's level of intention to quit smoking or to maintain non-smoking.

2. Feedback on the advantages (pros) and disadvantages (cons) of smoking and quitting smoking. Feedback is given on the respondent's scores on 12 items measuring the pros and cons of smoking.

3. Feedback on the respondent's perceived social influence (perceived social norms, social modelling and social support).

4. Feedback on level of self-efficacy not to smoke in potentially difficult situations, varying from not smoking when just having had dinner to not smoking when being offered a cigarette.

5. Feedback on action plans on the extent to which respondents are planning to undertake specific actions (e.g. telling others about one's quit attempt) while preparing their quit attempt.

6. Feedback on how to deal with difficult situations (coping plans). For the same situations as included in the questions on self-efficacy, respondents can choose to formulate a specific plan in order not to smoke when these situations occur. Respondents are asked to formulate these plans in the form of if-then statements, as these have proven to be successful in significantly reducing long-term relapse to smoking (van Osch et al., 2008).

7. Ending, in which the PAS research team wishes each respondent good luck during their quit attempt/maintaining their non-smoking behaviour.

The feedback letter respondents receive 2 days after the quit date they set at baseline consists of only one page as this feedback letter is based on a shorter questionnaire. This feedback provides respondents with relapse prevention strategies, as recent studies show that $70-80 \%$ of ex-smokers relapse within 3 months time (Hoving et al., 2006b). Respondents can access their personal feedback in three ways: it is directly made available online, feedback letters are sent to the respondent by email and they can be printed. The computer tailoring programme was pilot tested among both researchers specialized in smoking cessation research and among our target population of current smokers (Damoiseaux and van der Molen, 1998). Researchers were consulted for their expertise in translating theoretical concepts into questions, whereas smokers were asked about several characteristics of the questions and tailored feedback letters (e.g. Do you understand what is meant by each question?; Do you perceive the advice given as interesting?; Do you think the feedback messages are annoying?). The results from this pilot test indi- 
cated, among other things, that the questions were understandable and easy to answer and that the feedback given was clear and perceived as personally relevant. However, some minor technical changes had to be made in the tailoring programme (e.g. some people received feedback not completely matching their answers to the questions). An example of a tailored feedback message is provided in appendix 1 .

\section{Tailored counselling by practice nurses}

After receiving the first tailored feedback, respondents in the MTC group are prompted to schedule a counselling meeting with their practice nurse within 6-8 weeks. As shown in figure 2.1, the MTC group receives this counselling session at the same time as respondents in the MT group receive their third tailored feedback letter. Practice nurses are provided with a summary of the answers MTC respondents gave in the questionnaire at 6-week follow-up. The PAS counselling protocol and this summary together provide a framework for the counselling session. To assist practice nurses in guiding counselling sessions, a counselling protocol has been developed. This protocol is based on interviews held with practice nurses $(\mathrm{N}=16)$, to gain insight into their expectations and desires concerning the content and structure of such a counselling protocol. The final PAS counselling protocol consists of three main chapters guiding counselling sessions with three different types of patients: smokers who have quit, smokers who have quit but relapsed, and smokers who have not quit yet. For these three types of patients, different issues are to be discussed during the counselling session, but the structure of all sessions is the same. A pilot test has been conducted among practice nurses and smoking cessation counselling experts and has been modified based on their feedback. Both the content and structure of the different counselling sessions are visually displayed in figure 2.2. After each counselling session, practice nurses are asked to fill out a checklist, so that we can monitor what has been discussed in each session and investigate whether the PAS counselling protocol was used correctly. These checklists may be used by practice nurses during counselling sessions as a mnemonic device to prevent oversight. Practice nurses can return the checklists either by mail or e-mail. Pre-addressed and pre-paid envelopes have been included in the protocol for the practice nurses to return the checklists by mail. Moreover, general information about PAS and about the study being conducted, advice on how to recruit smoking patients for the study, a section with frequently asked questions and the PAS research team's contact information are also included in the counselling protocol. In addition, we provide practice nurses with a PAS CDROM. The CD-ROM visually supports the counselling protocol. 


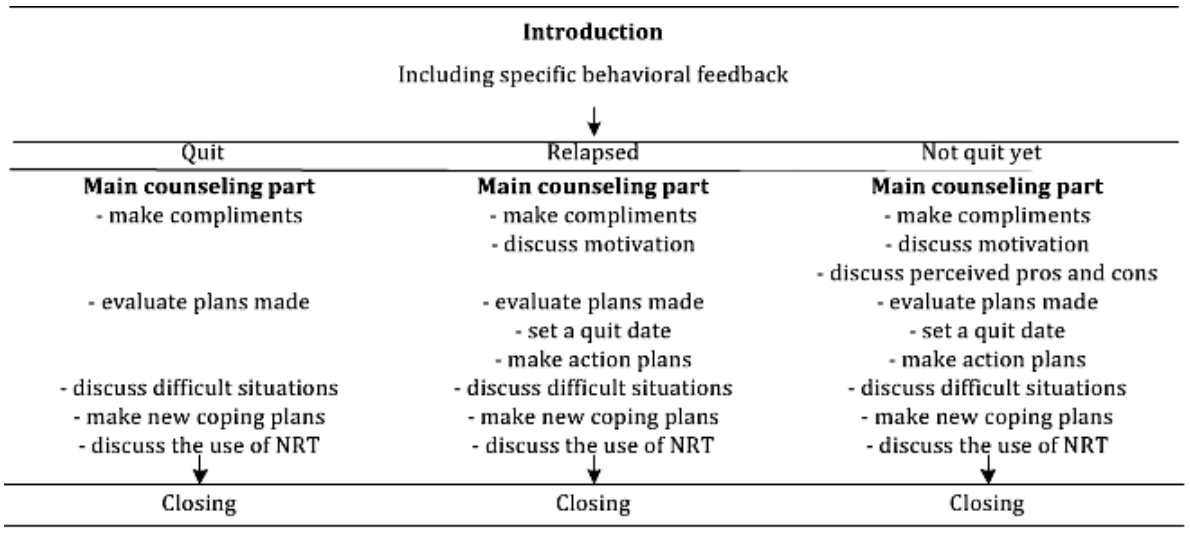

Figure 2.2 Flowchart of the three different types of counselling sessions by practice nurses.

Besides information about PAS, the CD-ROM also provides short exemplary videos presenting different types of smokers/ quitters a practice nurse may encounter in the general practice.

\section{Biochemical validation}

When conducting intervention studies, biochemical validation is warranted to test whether self-reported behaviour is accurate (Patrick et al., 1994). Mostly used is the cotinine assessment, as it is accurate and sample collection is simple: a sample of saliva can be collected with a swab stick. Practice nurses will be responsible for conducting cotinine tests in their general practice. As this test has to be undergone 4 days after filling out the last questionnaire at 12 months follow-up, the PAS research time will provide practice nurses with a sufficient number of cotinine tests beforehand. Once the respondent has filled out the twelve months follow-up questionnaires and indicated that he or she has quit smoking, the practice nurse will be instructed to invite the participant for a voluntary appointment to conduct the cotinine test. If respondents refuse to undergo the test, practice nurses are instructed to ask for their main reason for refusing. After the saliva is collected with a swab stick, it should be applied to a test strip, on which the test result will appear within 20 min. Results can be either negative (no cotinine measurable) or positive (cotinine detected in the saliva). Practice nurses will register test results for each patient and report the findings to the PAS research team both by e-mail and regular mail. After having combined the results with data previously gathered for the particular respondent, the saliva sample will be destroyed.

\section{Cohort retention}

To retain participating practice nurses, a range of strategies is used. Some of these are targeted, for instance calling them regularly to investigate their progress in 
recruiting patients and to discuss possibilities for improvement, and sending chocolate telegrams to each practice nurse who has recruited their first five smoking patients. Other strategies are more general, like sending newsletters outlining study progress and practice nurses' and patients' experiences with PAS. To retain respondents participating in the smoking cessation programme, each respondent will get a 10 Euro voucher after filling in the last questionnaire. This is mentioned at the beginning of each questionnaire in order to keep participants motivated to complete their participation in our study.

\section{Outcome measures}

The primary outcome measure is prolonged abstinence. Prolonged abstinence is defined as not having smoked since filling in the baseline questionnaire, taking into account a grace period of 4 weeks $(0=$ no; $1=y e s)$. A grace period is considered the initial period directly after the intervention delivery, in which quitting and reinitiating smoking behaviour will not be counted as such (Hughes et al., 2003, West et al., 2005).

\section{Secondary outcome measures}

Secondary outcome measures are having made a serious quit attempt (at least $24 \mathrm{~h}$ of abstinence) since the previous measurement point ( $0=$ no; $1=y e s), 24$-hour point prevalence abstinence (having refrained from smoking during the last 24 hours; $0=$ no; 1 =yes), 7 -day point prevalence abstinence (having refrained from smoking during the last 7 days; $0=$ no; $1=y e s$ ), continued abstinence (having refrained from smoking since the previous measurement point; $0=$ no; $1=y e s$ ) and changes in overall tobacco consumption, intention to quit smoking and/or to maintain nonsmoking, attitude, self-efficacy and social influence.

Overall tobacco consumption is measured using five open-ended questions regarding the quantity of cigarettes, shag, cigars, cigarillos and pipes respectively, smoked per day. The answers to these five questions are then converted into one overall score (in number of cigarettes) representing tobacco consumption.

Intention to quit smoking is measured by one item asking the respondent whether or not he or she is intending to quit smoking, on a 7-point Likert scale ( $1=$ very surely not; $7=$ very surely yes). This question is only asked when respondents indicate that they still smoke.

Intention to maintain non-smoking is measured by one item asking the respondent whether or not he or she is intending to maintain non-smoking, also on a 7point Likert scale ranging from $1=$ very surely not, to $7=$ very surely yes. This question is only asked when respondents have quit smoking.

Attitude is measured by twelve items, with which a respondent can agree or disagree, measured on a 5-point Likert scale (e.g. When I do not smoke, my condition will improve; $1=$ no, does not improve; $2=$ do not know; 3=yes, will improve a bit; 4=yes, will improve; 5=yes, will improve a lot; When I do not smoke, it is harder 
for me to relax; $1=$ no, it is not harder to relax; $2=$ do not know; $3=y e s$, it is a bit harder to relax; 4=yes, it is harder to relax; 5=yes, it is a lot harder to relax).

Self-efficacy is measured by nine items measured on a 5-point Likert scale (e.g. Do you think you will manage not to smoke when taking a break or when you are being offered a cigarette?; $1=$ surely not; $2=$ =probably not; $3=$ maybe yes, maybe no; $4=$ probably yes; $5=$ surely yes).

Social influence is measured by nine items: three items measuring social norms (e.g. My partner...; 1 =thinks that I should not smoke; $2=$ probably thinks that I should not smoke; $3=$ neutral/do not know; 4=probably thinks I should smoke; $5=$ thinks that I should smoke; $9=$ not applicable), three items measuring social support (e.g. My children support my not smoking; 1=no, my children do not support me; 2=do not know; 3=yes, my children support me a little; 4=yes, my children support me; $5=y e s$, my children support me a lot; $9=$ not applicable) and three items measuring social modelling (e.g. How many of your friends smoke?; 1=none of them; $2=$ the minority; $3=$ half of them; $4=$ the majority; $5=$ all of them; $9=$ not applicable). All three constructs take into account the respondent's partner, children and friends.

\section{Statistical analyses}

\section{Sample size and power}

We expect $10 \%$ point prevalence abstinence in the control condition (Dijkstra et al., 1998a, Hoving et al., 2010). Power estimations are based on the ability to detect differences of $10 \%$ with a power of .95 and p-value of .05 . We estimate that the multiple tailoring intervention will lead to $20 \%$ and the combination with counselling to $30 \%$ point prevalence abstinence. To be able to detect this difference significantly, almost 300 respondents per intervention arm are necessary at the end of the trial (900 respondents in total) . Including 20\% attrition over the trial period, 1100 respondents need to be recruited. As we expect an additional attrition of $20 \%$ in the multiple tailoring and counselling arm (as some respondents randomized in this arm might refuse to or do not show up), we aim to include around 1200 respondents in the study; 400 respondents in each of the study arms. As practice nurses will be recruiting 15 participants each, we have recruited 80 practice nurses. Given the high response rates in previous studies, the number of smoking participants and the number of practice nurses to be recruited are reasonable.

\section{Analysis}

As we randomize smoking patients within their general practices, to assess the effect of PAS on both main and secondary outcome parameters, multilevel analyses will be used. Conducting multilevel analyses will enable us to correct for the effect different practice nurses and/or general practices may have on the outcomes measured at patient level. As our main outcome parameters are dichotomous variables, logistic multilevel regression analyses will be conducted when it concerns these outcome measures. To assess the effect of PAS on secondary outcome meas- 
ures, linear multilevel regression analyses will be conducted, as these are continuous variables. Intention-to-treat (ITT) analysis will be conducted as well as complete case analysis. Results will be corrected for baseline variables (e.g. demographics, attitude, intention and smoking behaviour) by adding these variables to the multilevel model. Moreover, the likelihood of chance findings will be considered and if needed, Bonferonni corrections will be applied. Subgroup analyses will be conducted to analyze differences in the importance of the predictors in gender and SES (socioeconomic status) subgroups, also using multilevel analyses. These will be corrected for baseline variables (demographics, cognitions, intention and behaviour) by means of ANOVA. Bonferonni corrections will be applied if necessary. Data will be analyzed using Mplus version 5.

\section{Additional studies}

In addition to the effect study extensively described in this paper, three other studies will be conducted regarding PAS, which will be described briefly.

Process evaluation A process evaluation will be conducted among respondents, practice nurses and GPs to assess their respective experiences with PAS. In addition, this study will aim at identifying potential alterations that would improve the implementation of PAS. For respondents, questions about the intervention received will be added to the questionnaire they receive at 12-months follow-up. Practice nurses and GPs will be invited to fill in a process evaluation questionnaire included in the debriefing letter they receive at the end of the study.

Feasibility analysis Among those practice nurses initially labelled as nonadopters, a study will be conducted towards the feasibility of the diffusion of the PAS counselling protocol on a large scale. Additionally, we aim to identify possible improving alterations to the protocol in order to facilitate implementation. Within this study, practice nurses do not need to recruit participants, but are asked to use the PAS protocol for the duration of 3 months. After this period, they will be interviewed by telephone concerning their experiences, perceived advantages of the protocol and the difficulties encountered while using it. Moreover, the number of counselled smokers will be assessed.

Cost-effectiveness analysis In the end, a study will be conducted to assess the cost-effectiveness of the multiple computer tailoring programme and tailored counselling protocol, together entailing PAS.

\section{DISCUSSION}

This paper described the development of PAS and the design of the study testing its effectiveness. PAS aims to evaluate the effectiveness of a web-based multiple tailored smoking cessation programme combined with tailored counselling by practice nurses, compared with multiple tailoring alone and both compared with usual care. It is hypothesized that the combination of two effective stand-alone interventions will result in higher abstinence rates than either of these two alone. Within 
PAS the counselling session was provided by practice nurses instead of by GPs, as GPs have often reported a lack of time and skills to help smokers who want to quit (Hoving et al., 2007b, Yarnall et al., 2003). If counselling given by a practice nurse in combination with web-based computer tailoring is as effective, or even more effective, than counselling given by GPs, the GPs' time constraints could be reduced and practice nurses could permanently adopt the task of providing smoking cessation advice.

\section{(Potential) strengths of the study}

The present study had several potential strengths:

\section{Motivation as an inclusion criterion}

Only smoking patients are eligible to take part in PAS who are motivated to quit within 6 months. As there is only limited time and manpower available within the present study and as motivating smokers to quit can be very time-consuming when motivation is still low, this has led us to use motivation as an inclusion criterion. This is in accordance with inclusion criteria used in previous studies on the effect of online smoking cessation programme (Hoving, 2007, Te Poel et al., 2009). Moreover, smokers unmotivated to quit were not expected to be willing to set a quit date within the near future, one of the key elements of PAS.

\section{Randomization approach}

An important strength of our study is the randomization approach, in which allocation is covered and done by an automated computerized process. Randomization is done on the patient level, because of statistical considerations. Moreover, randomization on practice nurse level seemed unfavourable because it is likely that practice nurses who would have been allocated to the control group would have difficulties including and, more importantly, retaining, sufficient patients (Lindstrom et al., 2009).

\section{Use of checklists and audiotapes}

All participating practice nurses are given the same instructions on how to use the PAS counselling protocol. However, there is a possibility that not all practice nurses will use the protocol in exactly the same way, due for example to previously existing counselling habits or unfamiliarity with the new protocol. To be able to gain insight into the content of the consultations, all practice nurses are asked to fill out a checklist of each consultation they have according to the PAS protocol. This can be considered in line with the use of intervention cards in a previous study, a strategy proven to be an important predictor of adherence to a minimal-contact smoking cessation intervention (Segaar et al., 2007). Similarly to the so-called intervention cards, the PAS checklists can function not only as a registration system, but also as a schematic summary of the counselling protocol and as a reminder of all topics needing to be discussed. In addition, each practice nurse is asked to audiotape one of the 
PAS counselling sessions, to enable us to get insight into the quality of these sessions as well. Together, the checklists and audiotapes can be used to search for factors increasing or diminishing the effectiveness of the intervention.

\section{(Potential) limitations of the study}

The present study also had some potential limitations:

\section{Participant selection}

A selection bias may limit generalization of the results of this study. Those patients who do not have access to the Internet are excluded from participation, which could result in selection bias. However, in the Netherlands, almost $90 \%$ of the population has access to the Internet at home, which is the highest percentage of all countries in the European Union (Centraal Bureau voor de Statistiek, 2009). In addition, practice nurses are provided with a variety of recruitment materials, in order to make participation attractive for as wide a variety of patients as possible. Moreover, selective refusal of practice nurses is possible. To limit such bias' influence on the results and to create a relatively heterogeneous sample of practice nurses, a national sample of practice nurses has been recruited.

\section{Sample size calculation}

The sample size needed was calculated on the base of a Chi-square analysis, while for the main analyses regression analyses will be used. However, sample size calculations based on Chi-square analysis often overestimate the sample size needed, even though this will be (partly) undone by the multilevel structure of this study.

\section{Randomization on patient level}

Randomization on patient level could lead to contamination bias, as it is possible that practice nurses have consultations according the PAS protocol with patients from the MT or UC group. To minimize such bias, clear instructions to only use PAS intervention materials in the MTC group are given to each practice nurse before they start recruiting patients. When notifying practice nurses of a new patient participating in PAS, the group to which this patient has been allocated and what this implies for the care he or she should receive are indicated.

\section{CONCLUSION}

This extensive description of the development of PAS is provided in order to offer insight into its potentially effective working mechanisms. The results regarding the effectiveness of PAS, which will be reported in other papers, may contribute to the knowledge about effective elements of smoking cessation interventions aimed at smoking adults and about the possibilities of multiple computer tailoring combined with tailored counselling by practice nurses. 


\section{CHAPTER 3}

\section{Factors influencing Dutch practice nurses' intention to adopt a new smoking cessation intervention}

Published as: Leitlein, L., Smit, E.S., de Vries, H., Hoving, C. (2011). Factors influencingDutch practice nurses' intention to adopt a new smoking cessation intervention, Journal of Advanced Nursing, in press. 


\section{ABSTRACT}

Aims This article is a report of a study that aimed to identify factors influencing practice nurses' and nurse practitioners' intention to adopt a new smoking cessation intervention.

Background Although effective smoking cessation interventions exist and practice nurses can offer a considerable resource in advertising patients to quit smoking, due to several reasons the majority of practice nurses do not implement these interventions.

Methods A cross-sectional study was undertaken among Dutch practice nurses and nurse practitioners working in general practices $(n=139)$ using electronic questionnaires. Data were collected from January until March in 2009. T-tests were used to compare adopters with non-adopters about their predisposing and motivational factors. Logistic regression analyses were conducted to assess the variation in intention explained by these factors.

Results The majority of practice nurses did not intend to adopt the new intervention ( $\mathrm{n}=85$; $61.2 \%$ ). More practice nurses than nurse practitioners intended to adopt the intervention. Attitude and perceived social norms were found to be positively correlated with the intention to adopt the intervention whereas satisfaction with current smoking cessation activities was found to be negatively correlated.

Conclusion Important associations were found between profession, attitude, social norms and satisfaction, and the intention to adopt the new smoking cessation intervention. Practice nurses who do not intend to adopt need to be persuaded of the advantages of adopting. Perceived social norms need to be restructured and before presenting the intervention to a general practice current smoking cessation activities should be determined to increase the intervention's compatibility with these current practices. 


\section{INTRODUCTION}

Tobacco use is regarded as the most preventable cause of premature death in the world (WHO, 2008). Each year 5.4 million people die from the consequences of smoking, nevertheless, more than one billion people in the world continue using tobacco (WHO, 2008).

Although it is the individual smoker's decision to keep smoking or to quit, health care professionals can help and guide patients with an attempt to quit (Chavannes et al., 2007). In general, it appears to be effective if health care professionals, such as general practitioners and practice nurses, advise patients to stop smoking (van Weel et al., 2005), even brief behavioural counselling is effective in improving healthy behaviour (Fiore, 2000, Lancaster and Stead, 2005b, Steptoe et al., 1999). In the Netherlands the profession of practice nurses was introduced in 2001 as a new health care profession aimed at relieving GPs by taking over tasks such as providing care for the chronically ill (Derckx, 2006). Due to a positive nursepatient relationship (Shum et al., 2000, van Son et al., 2004) and to the large number of patients with smoking-related illnesses visiting general practices each year (van den Berg et al., 2003), practice nurses can offer a considerable resource in advising patients how to quit smoking.

\section{Background}

Although effective smoking cessation interventions exist (Fiore, 2000, Lancaster and Stead, 2005b, Pieterse et al., 2001, Van den Bruel et al., 2004, van Weel et al., 2005), the majority of practice nurses do not carry out these interventions (Chavannes et al., 2007, Eccles et al., 2007, Glasgow et al., 2003, Segaar et al., 2007). Reasons for not implementing smoking cessation interventions in the general practice are negative beliefs about smoking cessation interventions such as the belief that the intervention is not effective (Hall and Marteau, 2007, Hall et al., 2005), consumes too much time (Hall et al., 2005) or interferes with the health care professional-patient relationship (Chavannes et al., 2007, Coleman et al., 2001). Another group of impeding factors are structural and practical barriers, including lack of time (Hall and Marteau, 2007, Nagelhout, 2007), lack of finances (Needleman et al., 2006) and lack of social support (Nagelhout, 2007).

In the present study, the I-Change Model (De Vries et al., 2003) was used as a theoretical framework to assess the determinants of the intention to adopt a new smoking cessation intervention. This intervention (PAS, or Personal Advice in Stopping smoking) combines a web-based multiple computer tailored smoking cessation programme with a tailored counselling session with a practice nurse (Smit et al., 2010). According to the I-Change model, the intention to adopt PAS is determined by practice nurses' attitude, their perceived social influence and their selfefficacy. A positive attitude towards smoking cessation interventions in general and towards PAS in particular, is hypothesized to have an positive influence on the intention to adopt PAS. Similarly, when the social environment is perceived as posi- 
tive towards the adoption of PAS and, furthermore, when practice nurses feel confident (i.e. have a high self-efficacy) to adopt PAS in their general practice, the intention to adopt PAS will increase. These three motivational factors, in turn, are predicted by several predisposing factors, such as awareness of PAS' existence and practice nurses' knowledge about the content of PAS.

Besides these well-investigated factors influencing the adoption of smoking cessation programmes, there is another possible determinant. Past research has reported much about patients' satisfaction regarding consultations with practice nurses; compliance rises if the patient is satisfied with the consultation (Shum et al., 2000, van Son et al., 2004, Derckx, 2006). There is, however, a shortage of research about practice nurses' satisfaction with the current smoking cessation activities that they are involved in. Practice nurses' dissatisfaction with their current situation might improve the chance of adoption of new smoking cessation interventions. Supported by other adoption studies investigating the determinants of adopting a smoking cessation intervention by health care professionals (Bolman et al., 2002a, Hall et al., 2005) and the assumptions of the I-Change model, it can be expected that a practice nurse's high dissatisfaction with current smoking cessation activities is related to a more positive attitude towards PAS and a higher intention to adopt PAS.

\section{THE STUDY}

Aim

In this study, we aimed to provide insight into which theoretical concepts determined practice nurses' intention to use PAS in their practice.

\section{Design}

A cross-sectional study was carried out.

\section{Sample/Participants}

Practice nurses and nurse practitioners working in Dutch general practices were asked to participate in the study. In the Netherlands, practice nurses have an intermediate level of vocational education and most often provide care for patients with chronic diseases such as diabetes, asthma, or hypertension (Nederlands Huisartsen Genootschap and Landelijke Huisartsen Vereniging, 2011). Nurse practitioners have a higher vocational educational level and usually have more responsibilities, e.g. preventive care and care for elderly patients (Nederlands Huisartsen Genootschap and Landelijke Huisartsen Vereniging, 2011). Recruitment was conducted through thirteen organizations for practice nurses and/or general practitioners, altogether having a potential reach of at least 1800 practice nurses and over 18.000 general practitioners. According to the organizations' preferences, a recruitment letter was sent to their members through their website, (electronic) newsletter or personal email correspondence. In addition, members of the research team personally approached practice nurses with previous interest in PAS, a PAS website was devel- 
oped and a Dutch social networking website that includes groups of practice nurses agreed to send a recruitment letter to all its members. After agreeing to participate, all practice nurses were asked to fill out an online questionnaire. Because of the open source character of the questionnaire it was not possible to detect the questionnaire's response rate, but in total 239 questionnaires were returned. This is compared to the 138 that would have already been sufficient to detect a .5 difference between adopters and non-adopters with regard to their score on the main outcome measure, i.e. their intention to adopt PAS (DSS Research).

\section{Data collection}

Data was collected from January until March in 2009. The electronic questionnaire consisted of 67 questions regarding demographic characteristics, satisfaction, attitude, social influence, self-efficacy, and intention. Based on a pilot test, it was estimated that completing the questionnaire would last 15 minutes. At the start of the online questionnaire, respondents were asked to indicate their profession; respondents who were not practice nurses or nurse practitioners were excluded.

\section{Ethical considerations}

PAS was approved by the Medical Ethics Committee of Maastricht University and the University Hospital Maastricht (MEC 08-3-037; NL22692.068.08), and is registered with the Dutch Trial Register (NTR1351).

\section{Data analysis}

The analyses were conducted with SPSS version 15.0 (SPSS, Chicago, IL, USA). Effects were considered significant when $\mathrm{p}<.05$. Totally, the questionnaire consisted of 67 questions.

First, a missing value analysis was conducted; respondents who had more than $20 \%$ missing values were excluded from further analyses.

Respondents were divided in two groups based on their intention to adopt PAS ("Does your general practice intend to participate in the study?"); non-adopters (no, probably not or maybe) and adopters (most probably and yes). T-tests were conducted to evaluate differences between adopters and non-adopters on demographics, the three motivational factors attitude, social influence and self-efficacy and satisfaction with current smoking cessation activities.

Logistic regression analysis using the enter method was used to assess which determinants could, in combination, best explain the practice nurses' intention to adopt PAS. Four blocks were included. The first block consisted of demographic variables to determine which demographics significantly explained the intention to adopt PAS. In the second block, satisfaction was entered in order to examine whether or not this variable could explain the variance in intention to adopt PAS. The third block consisted of demographics, satisfaction and the three intrapersonal determinants; attitude, social influence and self-efficacy. In order to assess whether 
satisfaction might moderate the relationship between attitude and intention, an interaction term was added in the fourth block.

\section{Validity and reliability}

Demographics were measured by 14 items. With regard to the general practice these variables included location, practice setting (rural, semi-urban or urban), total amount of registered patients and total number of GPs, practice nurses and assistants working in the general practice. Additional individual characteristics assessed were workload, years of experience working as a practice nurse, years working in the current general practice, age, gender, smoking status (non-smoker or smoker) and years of experience in giving smoking cessation advice.

Respondents were also asked about their satisfaction with current smoking cessation activities. This was measured by four questions ranging from 1 (totally disagree) to 5 (totally agree), which together formed one factor $(\alpha=0.86)$.

Attitude was measured by 12 questions using a five-point Likert scale ranging from 1 (totally disagree) to 5 (totally agree). Factor analyses and reliability analyses revealed two subscales. The first subscale, attitude towards smoking cessation interventions in general $(\alpha=0.96)$, consisted of three questions. The second subscale measured the attitude towards PAS $(\alpha=0.86)$ and consisted of nine questions.

In order to assess the social influence perceived by practice nurses related to smoking cessation interventions, seven questions were included. Based on factor analyses and past research these questions were divided into subscales consisting of three items measuring social influence with regard to smoking cessation interventions in general $(\alpha=0.89)$, two items on existing social norms with regard to PAS $(\alpha=0.82)$ and two items assessing social support with regard to PAS $(\alpha=0.60)$. Questions measuring the social influence with regard to smoking cessation interventions and social support with regard to PAS were coded from 1 (totally disagree) up to 5 (totally agree). Questions regarding existing social norms concerning PAS ranged from 1 (totally negative) up to 5 (totally positive).

Self-efficacy concerning PAS was evaluated by three questions, which formed one factor $(\alpha=0.68)$ and were ranged from 1 (very unlikely) to 5 (very likely).

One question measured the practice nurses' intention to participate in the research project regarding the effect of PAS. This dependent variable was assessed on a five-point scale ( $1=$ no, $2=$ probably not, $3=$ maybe, $4=$ most probably, $5=y e s$ ).

Table 3.1 provides an overview of the concepts measured and provides some examples of the questions included in the questionnaire to measure these concepts. In addition, a description is provided of the (sub)scales formed, together with their relative reliability. 


\section{RESULTS}

\section{Characteristics of the sample}

Due to a high number of missing values (> 20\%) 81 out of 239 questionnaires were not appropriate to use for further analyses and were thus excluded. Another 17 questionnaires were filled out by other professions than practice nurses or nurse practitioners and were also excluded. Two questionnaires missed an answer to the item regarding the intention to adopt PAS and were excluded from the analyses as well. In total, 100 questionnaires were excluded from further analyses, resulting in a final sample of 139 practice nurses.

Characteristics of the final sample $(n=139)$, also divided in potential adopters $(n=54)$ and non-adopters $(n=85)$, are presented in table 3.2. The majority of respondents were female (97.1\%) practice nurses with an average age of 44 years $(\mathrm{SD}=8.18)$. The mean working experience as a practice nurse or nurse practitioner was four years $(\mathrm{SD}=2.89)$ and experience in giving smoking cessation advice three years ( $S D=2.61)$. Most of the practice nurses were non-smokers $(49.6 \%)$ or exsmokers (48.9\%). Respondents reported working in the current general practice for an average of six years $(S D=5.92)$ with an average of 21 hours per week $(S D=7.31)$. The average general practice included four GPs, one practice nurse and five assistants and included over 4500 registered patients.

\section{Differences between adopters and non-adopters}

There was a significant relationship $(\mathrm{p}<.05)$ between the function in the general practice and whether or not respondents intended to adopt PAS. More practice nurses $(40.5 \%)$ than nurse practitioners $(14.3 \%)$ indicated to intend to adopt PAS.

Differences regarding demographics, attitudes, social influence, self-efficacy and satisfaction are presented in table 3.2. Adopters presented a significantly more positive attitude and perceived social influence, including social norms and social support, towards PAS than non-adopters. Furthermore, adopters reported significantly less satisfaction with regard to current smoking cessation activities they were involved in than non-adopters.

No significant differences between potential adopters and non-adopters were observed for demographics regarding the general practice and individual characteristics, attitude and social influence towards smoking cessation interventions in general and self-efficacy towards PAS. 
Table 3.1 Measures of attitude, social influence, self-efficacy and satisfaction and their reliability

\begin{tabular}{|c|c|c|c|}
\hline & $\begin{array}{c}\text { Number of } \\
\text { items }\end{array}$ & Example questions & Cronbach's $\alpha$ \\
\hline \multicolumn{4}{|l|}{ Attitude } \\
\hline $\begin{array}{l}\text { towards smoking cessation } \\
\text { interventions in general }\end{array}$ & 3 & $\begin{array}{l}\text { "I consider smoking cessation advice to be } \\
\text { important" }\end{array}$ & 0.96 \\
\hline towards PAS & 9 & $\begin{array}{l}\text { "PAS is an effective smoking cessation } \\
\text { intervention" }\end{array}$ & 0.86 \\
\hline \multicolumn{4}{|l|}{ Social influence } \\
\hline $\begin{array}{l}\text { towards smoking cessation } \\
\text { interventions in general }\end{array}$ & 3 & $\begin{array}{l}\text { "My colleagues consider smoking cessation } \\
\text { advice to be important" }\end{array}$ & 0.89 \\
\hline perceived support to adopt PAS & 2 & "My colleagues will stimulate the use of PAS" & 0.60 \\
\hline perceived norms to adopt PAS & 2 & $\begin{array}{l}\text { "What do you think is your colleague's attitude } \\
\text { towards PAS?" }\end{array}$ & 0.82 \\
\hline \multicolumn{4}{|l|}{ Self-efficacy } \\
\hline to use PAS & 3 & "I can persuade my patients to stop smoking" & 0.68 \\
\hline \multicolumn{4}{|l|}{ Satisfaction } \\
\hline with current practices & 4 & $\begin{array}{l}\text { "I am satisfied with the amount of smoking } \\
\text { cessation advice we offer" }\end{array}$ & 0.86 \\
\hline
\end{tabular}


Table 3.2 Descriptives of practice nurses' demographics, their attitude, social influence and self-efficacy beliefs and satisfaction with current smoking cessation activities

\begin{tabular}{|c|c|c|c|c|c|c|}
\hline & Total & $\begin{array}{l}\text { Adopters } \\
\text { (54) }\end{array}$ & $\begin{array}{c}\text { Non-Adopters } \\
\text { (85) }\end{array}$ & $\mathrm{T}$ & $\chi^{2}$ & $\mathrm{p}$ \\
\hline $\begin{array}{l}\text { Number (\%) of practice nurses working } \\
\text { in a ... general practice }\end{array}$ & & & & & 0.90 & 0.68 \\
\hline Rural & $55.00(39.6)$ & $22.00(40.7)$ & $33.00(38.8)$ & & & \\
\hline Semi-Urban & $24.00(17.3)$ & $11.00(20.4)$ & $13.00(15.3)$ & & & \\
\hline Urban & $60.00(43.2)$ & $21.00(38.9)$ & $39.00(45.9)$ & & & \\
\hline \multicolumn{7}{|l|}{ Mean (SD) number of } \\
\hline Registered patients & $\begin{array}{c}5,320.00 \\
(3,252.58)\end{array}$ & $\begin{array}{c}5,649.00 \\
(3,898.26)\end{array}$ & $\begin{array}{c}5,107.00 \\
(2,759.58)\end{array}$ & -0.94 & & 0.35 \\
\hline GPs working in the practice & $4.00(2.30)$ & $4.00(2.71)$ & $3.00(2.01)$ & -0.99 & & 0.33 \\
\hline Practice nurses working in the practice & $1.00(1.08)$ & $1.00(1.23)$ & $1.00(0.94)$ & -1.42 & & 0.16 \\
\hline Assistants working in the practice & $5.00(3.42)$ & $5.00(5.44)$ & $4.00(4.46)$ & -1.50 & & 0.13 \\
\hline Number (\%) of & & & & & 6.21 & 0.02 \\
\hline Practice nurses & $85.00(61.2$ & $40.00(74.1)$ & $45.00(52.9)$ & & & \\
\hline Nurse practitioners & $54.00(38.8)$ & $14.00(25.9)$ & $40.00(47.1)$ & & & \\
\hline Number (\%) of & & & & & 2.62 & 0.16 \\
\hline Males & $4.00(2.9)$ & $0.00(0.0)$ & $4.00(4.7)$ & & & \\
\hline Females & $135.00(97.1)$ & $54.00(100.0)$ & $81.00(95.3)$ & & & \\
\hline Number (\%) of & & & & & 3.89 & 0.21 \\
\hline Non-smokers & $69.00(49.6)$ & $23.00(42.6)$ & $46.00(54.1)$ & & & \\
\hline Ex-smokers & $68.00(48.9)$ & $30.00(55.6)$ & $38.00(44.7)$ & & & \\
\hline Social smokers & $1.00(0.7)$ & $1.00(1.9)$ & $0.00(0.0)$ & & & \\
\hline Smokers & $1.00(0.7)$ & $0.00(0.0)$ & $1.00(1.2)$ & & & \\
\hline Mean (SD) workload in hours/week & $21.00(7.34)$ & $21.00(7.77)$ & $20.83(7.09)$ & -0.13 & & 0.90 \\
\hline \multicolumn{7}{|l|}{ Mean (SD) years working } \\
\hline As practice nurse/nurse practitioner & $4.00(2.89)$ & $3.94(2.69)$ & $4.15(3.02)$ & 0.42 & & 0.67 \\
\hline In current practice & $6.00(5.92)$ & $5.55(5.60)$ & $5.90(6.14)$ & 0.34 & & 0.74 \\
\hline $\begin{array}{l}\text { Mean (SD) years of experience in } \\
\text { smoking cessation advice }\end{array}$ & $3.00(2.61)$ & $2.81(2.35)$ & $3.20(2.76)$ & 0.84 & & 0.40 \\
\hline Mean (SD) age & $44.00(8.18)$ & $42.81(8.75)$ & $44.05(7.80)$ & 0.86 & & 0.39 \\
\hline \multicolumn{7}{|l|}{ Mean (SD) attitude (range 1-5) } \\
\hline Towards smoking cessation interventions & $4.78(0.69)$ & $4.69(0.91)$ & $4.84(0.51)$ & 1.12 & & 0.27 \\
\hline Towards PAS & $3.47(0.51)$ & $3.81(0.40)$ & $3.26(0.45)$ & -7.36 & & $<0.01$ \\
\hline \multicolumn{7}{|l|}{ Mean (SD) social influence (range 1-5) } \\
\hline Towards smoking cessation interventions & $4.47(0.84)$ & $4.46(0.98)$ & $4.78(0.74)$ & 0.15 & & 0.88 \\
\hline Norms towards PAS & $3.97(0.73)$ & $4.31(0.70)$ & $3.76(0.64)$ & -0.71 & & $<0.01$ \\
\hline Support towards PAS & $2.98(0.49)$ & $3.11(0.60)$ & $2.90(0.38)$ & -2.32 & & 0.02 \\
\hline Mean (SD) self-efficacy (range 1-5) & $3.88(0.64)$ & $3.99(0.53)$ & $3.80(0.69)$ & -1.76 & & 0.08 \\
\hline Mean (SD) satisfaction (range 1-5) & $3.46(0.89)$ & $3.24(0.85)$ & $3.60(0.89)$ & 2.36 & & 0.02 \\
\hline
\end{tabular}

Note: p-values <.05 are marked bold 


\section{Explanation of intention towards the adoption of PAS}

The final model of the logistic regression explained $56.2 \%$ of the variance in intention to adopt PAS. Attitude and social norms towards PAS showed to be of significant positive influence in determining the respondent's intention to adopt PAS. In relation to the earlier models the significance of profession and satisfaction disappeared after adding the concepts of attitude, social influence and self-efficacy. In fact, the relation between profession respectively satisfaction and the intention towards the adoption of PAS was mediated by these motivational factors. No significant results were found for attitude and social influence towards smoking cessation interventions in general, social support towards PAS and self-efficacy. An interaction between attitude towards PAS and satisfaction with current smoking cessation activities did not significantly determine intention to adopt $(\mathrm{OR}=6.81 ; 95 \%$ $\mathrm{CI}=0.93-49.83)$. Specific information about the logistic regression analysis is shown in table 3.3.

Table 3.3 Logistic regression of determinants of intention to adopt PAS

\begin{tabular}{|c|c|c|c|c|}
\hline & \multirow[b]{2}{*}{ Odds Ratio } & \multirow[b]{2}{*}{ p-value } & \multicolumn{2}{|c|}{$95 \%$ Confidence Interval } \\
\hline & & & Lower & Upper \\
\hline \multicolumn{5}{|l|}{ Block 1} \\
\hline Profession & 2.50 & 0.04 & 1.03 & 6.03 \\
\hline \multicolumn{5}{|l|}{ Block 2} \\
\hline Profession & 2.80 & 0.03 & 1.11 & 7.11 \\
\hline Satisfaction & 0.51 & 0.01 & 0.31 & 0.84 \\
\hline \multicolumn{5}{|l|}{ Block 3} \\
\hline Profession & 1.69 & 0.42 & 0.48 & 5.94 \\
\hline Satisfaction & 0.66 & 0.18 & 0.36 & 1.21 \\
\hline \multicolumn{5}{|l|}{ Attitude } \\
\hline general & 0.48 & 0.33 & 0.11 & 2.06 \\
\hline PAS & 100.41 & 0.00 & 10.65 & 947.24 \\
\hline \multicolumn{5}{|l|}{ Social influence } \\
\hline general & 0.77 & 0.62 & 0.27 & 2.16 \\
\hline PAS norms & 4.76 & 0.00 & 1.84 & 12.32 \\
\hline PAS support & 1.10 & 0.91 & 0.22 & 5.58 \\
\hline \multicolumn{5}{|l|}{ Self-efficacy } \\
\hline PAS & 1.00 & 0.98 & 0.32 & 3.03 \\
\hline
\end{tabular}

Note: p-values $<.05$ are marked bold.

\section{DISCUSSION}

\section{Study limitations}

This study is subject to some limitations. That is, this study had a cross-sectional design, implying that there was only one measurement per respondent at one point in time (Bouter et al., 2005). Consequently, it was impossible to reach a conclusion 
about causality and this study needs to be followed up by longitudinal studies. Because of the open character of the online questionnaire, response rates could not be defined and characteristics of non-responders could not be assessed. Furthermore, the study was conducted amongst Dutch practice nurses and nurse practitioners, investigating their intention to adopt an intervention developed and yet available in the Netherlands only. Therefore, some caution is warranted when generalizing the findings from our study to other professions or to practice nurses and nurse practitioners working in other countries. Lastly, when dichotomous categories were made for the intention to adopt PAS, the category non-adopters consisted of respondents with no intention to adopt PAS and of respondents who were possibly intending to adopt PAS (i.e. who answered maybe when asked whether they intended to adopt PAS), resulting in a relatively heterogeneous group of people. Although the main objective of the present study was to study the determinants of the intention to adopt PAS, and not of the possible intention to adopt PAS, it could be argued that the group of practice nurses who were possibly intending to adopt PAS could differ from non-adopters (Hoving et al., 2006a). However, our study did not have a sufficient number of respondents to adequately assess the potential differences between non-adopters and those possibly intended to adopt.

\section{Discussion of results}

The present study provides important results concerning the association between the intention to adopt the new smoking cessation intervention PAS and its possible determinants. The most important findings were that attitude and social norms towards PAS were positively related to the intention to adopt PAS and that satisfaction with current smoking cessation activities was negatively relation to the intention to adopt. No relation was found with self-efficacy. Profession was the only demographic characteristic influencing the intention to adopt PAS; more practice nurses than nurse practitioners intended to do so.

A minority of the participating practice nurses and nurse practitioners (39\%) reported that they were intending to adopt PAS in the future. Although other studies reported more willingness among general practice staff to adopt a smoking cessation intervention (Applegate et al., 2007, Nagelhout, 2007), these focused on a sample which also included GPs or specialists. It is possible that GPs and specialists show a higher intention to adopt the smoking cessation interventions studied, but then delegated the execution to their staff, including assistants, practice nurses or nurse practitioners. The results presented in this article might therefore be more realistic regarding the feasibility of implementing PAS in Dutch general practice.

The current study used a theoretical framework (the I-Change model) which also functioned as a basis for previous studies regarding the adoption of lifestyle change interventions in Dutch general practices (De Vries et al., 2003, de Vries and Mudde, 1998, Lee, 2004, Nagelhout, 2007). The model seemed to be valid in predicting the intention to adopt a new smoking cessation intervention among Dutch practice nurses as well. Results concerning the main concepts from the I-Change 
model that were studied, i.e. attitude, social influence and self-efficacy, are described in detail below.

\section{Attitude towards PAS}

Attitude towards PAS was positively related to PAS' rate of adoption. Previous studies confirmed that a positive view on the smoking cessation intervention determines its rate of adoption among general practice staff (Applegate et al., 2007, Clark et al., 2008, Heyes et al., 2004, Kaner et al., 2007, McEwen and West, 2001, Nagelhout, 2007, Sinclair et al., 2008).

\section{Self-efficacy to adopt PAS}

Contrary to other adoption studies (Applegate et al., 2007, Nagelhout, 2007, Sinclair et al., 2008, Wetta-Hall et al., 2005), self-efficacy seemed to have no influence on the intention to adopt PAS. It should be recognised that self-efficacy was only measured with three items. It is possible that the results of this study were different, if there were more items for self-efficacy. Nevertheless, most of the respondents felt confident talking with patients about smoking cessation and persuading them to stop smoking. A possible reason for not finding a significant effect of self-efficacy could be that PAS was developed based on the needs of practice nurses and that PAS' requirements on practice nurses are not demanding (Smit et al., 2010). Therefore, ideally practice nurses should feel confident in adopting PAS and, as a result, the total sample's scores on self-efficacy were expected to be high.

\section{Perceived social influence to adopt PAS}

Although differences between adopters and non-adopters were found in perceived social norms and social support, only social norms seemed to explain the intention to adopt PAS when other determinants were included in the analysis. Other studies also indicated that important others, such as patients and colleagues, play a crucial role in the adoption process of smoking cessation interventions (Coleman et al., 2001, Hall and Marteau, 2007, Kaner et al., 2007, Nagelhout, 2007, Sinclair et al., 2008). One possible explanation for the non-significance of social support is linked to the high practice nurses' self-efficacy regarding the adoption of PAS. If practice nurses feel confident with the adoption of PAS, their colleagues' social support might not be needed. Moreover, in comparison to social support, norms are a more indirect kind of social influence, as they are perceived by persons, but do not automatically reflect the truth (Brug et al., 2008). It is possible that negative social norms ("My colleague's attitude towards PAS is totally negatively") were perceived by the practice nurses, although the colleagues might have thought positively about PAS. This means that a discrepancy between perceived social norms and the real opinion of their colleagues may have existed. 
Satisfaction with current smoking cessation activities and the intention to adopt PAS If practice nurses and nurse practitioners were dissatisfied with their involvement in and the quality of current smoking cessation activities, they were more likely to intend to adopt PAS than if they were satisfied. Moreover, the effect of satisfaction with current smoking cessation activities seemed to be mediated by attitude. This finding seemed to be connected to the principles of persuasion. Rossiter et al. (1987) suggest that persuasion intention originates from different intrapersonal motives. Incomplete satisfaction is one of these motives or conditions, which people try to remove (Rossiter and Percy, 1987). Practice nurses who perceived dissatisfaction with their smoking cessation activities might therefore have been more likely to report a higher intention to adopt PAS.

\section{Conclusion}

This study has identified a number of determinants which are important in practice nurses' decisions whether or not to adopt a smoking cessation intervention. It seems that potential adopters of PAS can be characterized as having a more positive attitude towards PAS, as well as experiencing a higher social acceptance concerning the adoption of PAS. The results presented in this paper have several implications for both practice and research.

First of all, practice nurses who did not intend to adopt PAS need to be persuaded of the advantages of adopting PAS. Therefore, a more positive attitude towards PAS should be reached, e.g. by increasing the triability of PAS and by making PAS more visible for practice nurses. This might be realized using other practice nurses or patients reporting positive experiences with PAS. Moreover, it may be necessary to reward general practices more directly if they want to adopt PAS, for example by providing them with financial incentives and study credit. To improve social norms, steps can be taken to identify key players among practice nurses and focus recruitment strategies towards them specifically.

Secondly, further research should aim to examine whether satisfaction influences intention in a direct or indirect way. In the current study, satisfaction seemed to be mediated by attitude. As this study had a cross-sectional design, longitudinal research is needed to determine whether mediation really occurs, what role satisfaction plays when added to the I-Change model and how its relations with other factors in the model are.

Thirdly, although much research has been done on hospital nurses' or GPs' attitude, social influence and self-efficacy towards smoking cessation interventions (Bolman et al., 2002a, Johnston et al., 2004, Lambert et al., 2005, McCarty et al., 2001, Sarna et al., 2001, Segaar et al., 2006, Ulbright et al., 2006, Whyte et al., 2006), this is one of the first studies targeting practice nurses. As a result, further research is needed focusing on this specific target group to falsify or verify the results presented.

Lastly, before introducing PAS or similar new smoking cessation interventions into general practices it is important to assess satisfaction with current smoking 
cessation activities in these practices. When practice nurses are dissatisfied with current activities, new interventions can be developed adapted to practice nurses' needs with regarding to smoking cessation intervention aids. 


\section{CHAPTER 4}

Influence of recruitment strategy on the reach and effect of a web-based multiple computer-tailored smoking cessation intervention amongst Dutch adult smokers

Published as: Smit, E.S., Hoving, C., Cox, V.C.M., de Vries, H. (2011). Influence of recruitment strategy on the reach and effect of a web-based multiple tailored smoking cessation intervention amongst Dutch adult smoker, Health Education Research, 27(2):191-9. 


\section{ABSTRACT}

Introduction This study investigated the influence of two different recruitment strategies on the reach and effect of a web-based multiple tailored smoking cessation programme.

Methods From May 2009 until June 2010, Dutch adult smokers were recruited via mass media or general practices. Those who completed the baseline questionnaire were followed up during six weeks (two follow-ups). Differences between the two samples were assessed regarding baseline characteristics, retention rates, quit attempts and 24-hour point prevalence abstinence.

Results Smokers recruited via general practices $(\mathrm{N}=409)$ were significantly lower educated, less addicted, more motivated to quit smoking and to maintain non-smoking, more often female and more often suffering from cardiovascular or respiratory diseases than mass media respondents $(\mathrm{N}=1154)$. They showed higher retention rates and were more likely to report a quit attempt (64.3 vs. $50.7 \%$ ) and abstinence ( 43.3 vs. $33.1 \%$ ).

Conclusion More respondents could be recruited via mass media, while general practices respondents showed higher retention rates and were more successful in quitting smoking. The choice for a particular recruitment strategy appeared to determine the number and type of smokers recruited and might consequently influence the intervention's potential public health impact. 


\section{INTRODUCTION}

The smoking of tobacco is the most preventable cause of illness and premature death in the world (USDHHS, 2004, WHO, 2008). To aid smokers to quit successfully, effective computer tailored smoking cessation programmes have been developed (Lancaster and Stead, 2005c, Noar et al., 2007) that are increasingly delivered online (Webb et al., 2010, Lustria et al., 2009). Using the Internet as a medium for delivering these programmes has clear advantages for both provider and receiver. The Internet enables health promoters to reach a large audience against minimal costs and for participants these programmes are convenient as they can take part at any time their schedule and mood allows it (Griffiths et al., 2006). A recent review showed that smoking cessation interventions delivered through the Internet can indeed be effective (Civljak et al., 2010, Shahab and McEwen, 2009). However, the public health impact of these interventions is not only dependent on their effect, but also on their reach (Velicer and Prochaska, 1999).

Now that the Internet is becoming an increasingly popular medium for delivering health behaviour change programmes, it is important to investigate how recruitment and retention rates for web-based interventions can be optimized. Although online advertising has been suggested to be able to achieve relatively high recruitment rates (Graham et al., 2008), it is not yet clear whether all segments of the smoking population, such as smokers with a low socio-economic status (Brouwer et al., 2010, Lorence and Park, 2008, van Dijk, 2003), can be successfully reached via the Internet. Previously, recruitment via general practices has been shown to be successful in reaching smokers with a low socio-economic status for participation in a computer tailored smoking cessation intervention (Hoving et al., 2007a), but whether recruitment via (online) mass media is able to match this ability remains yet unknown.

The present study investigated whether recruitment via general practices and recruitment via (online) mass media resulted in different samples of smokers, with regard to size and demographic characteristics, participating in a web-based multiple tailored smoking cessation programme. In addition, potential differences between the two samples in retention rates and in smoking behaviour measured after a short follow-up period were examined.

\section{METHODS}

\section{Recruitment and procedure}

From May 2009 until June 2010, smokers aged 18 years or older were recruited to participate in a randomized controlled trial to test the effectiveness of a web-based multiple computer tailored smoking cessation programme. Smokers motivated to quit within six months, able to understand Dutch sufficiently, and with access to the internet were eligible for participation. Two recruitment strategies were used. First, recruitment via 80 practice nurses working in Dutch general practices. All practice nurses were provided with instructions on how to recruit their smoking patients. 
Desk displays, recruitment letters, recruitment texts for general practices' websites, business cards, and posters were available and incentives were sent to each practice nurses who had recruited five and ten patients, respectively. On average, $€ 6266,40$ was spent on recruitment via general practices (€78,33 per practice). Secondly, recruitment took place via mass media channels, such as regional newspapers, advertisements, an online social network website (Hyves) and online smoking cessation forums. In total, €1190 was spent (all on advertising).

After giving informed consent, participants were randomly allocated to either the control condition (i.e. usual care) or an experimental condition. Randomization took place at respondent level and was done by a computer software randomization device.

\section{Intervention}

The web-based multiple computer-tailored smoking cessation programme was based on the I-Change model (De Vries et al., 2003)and on a previously developed effective single computer-tailored intervention (Dijkstra et al., 1998a). All respondents were prompted by e-mail to fill in an online questionnaire at baseline, two days after a set quit date, and at six-week follow-up. One week after receiving their first invitation, a reminder e-mail was sent to respondents who had not yet filled out the particular follow-up questionnaire. For respondents in the intervention group, questions were interchanged with tailored feedback messages to maintain the respondent's attention and to improve retention rates. Feedback messages were tailored to several respondent characteristics (De Vries et al., 2003) such as gender, (non-)smoking-related beliefs (i.e. attitude, social influence and self-efficacy), intention to quit smoking, goal and relapse prevention strategies (i.e. action and coping plans), and smoking behaviour. After completion of each questionnaire, feedback messages were combined into a personalized feedback letter. These letters were available for respondents on their computer screen and were sent to them by email. A more extensive description of the intervention components is provided elsewhere (Smit et al., 2010).

\section{Questionnaire}

Data collection occurred online. After completing the baseline questionnaire, all respondents were prompted by e-mail to fill in two online follow-up questionnaires: two days after the quit date each respondent was asked to set at baseline and at six-week follow-up. Questionnaires were based on the I-Change model (De Vries et al., 2003) and tested experimentally in previous studies among Dutch smoking adults (Dijkstra et al., 1996, Dijkstra et al., 1998a, Hoving et al., 2007a).

\section{Baseline measurement}

Four demographic variables were measured at baseline: age, gender, educational level and nationality. 
The occurrence of cardiovascular or respiratory diseases, diabetes or cancer was measured by four dichotomous items, asking whether the respondent suffered from these diseases or not. Whether the respondent was pregnant was measured by one dichotomous item.

Addiction level was measured by the abbreviated Fagerström Test for Nicotine Dependence (FTND) ( $0=$ not addicted); $10=$ highly addicted) (Heatherton et al., 1991).

Number of previous quit attempts was assessed by asking respondents how often they had tried to quit smoking in the past.

Intention to quit smoking was measured by one item asking respondents whether or not they intended to quit smoking (1=definitely not; 7=definitely yes).

Intention to maintain non-smoking was measured by one item asking respondents if they intended to maintain non-smoking once quit (1=definitely not; $7=$ definitely yes).

\section{Follow-up measurement}

Two days after their set quit date, respondents were asked whether they had undertaken a quit attempt or not. Retention at this first follow-up was defined as still being in the study, measured by whether or not the respondent filled out this follow-up questionnaire.

At six-week follow-up, 24-hour point prevalence abstinence was assessed by asking whether the respondent had refrained from smoking during the last 24 hours. Retention was again defined as still being in the study, measured by whether or not the respondent filled out the six-week follow-up questionnaire.

\section{Analyses}

Data were analyzed using SPSS 15.0. Baseline differences between the two groups were assessed using Chi-square tests (for categorical variables) and T-tests (for interval variables), including all respondents who initially signed up for the study.

To investigate the influence of recruitment method on retention rates, logistic regression analyses were conducted with retention at the first and second follow-up as dependent variables, including all respondents who met inclusion criteria. First, we aimed to univariately assess the influence of recruitment method on retention. Therefore, the first model only consisted of recruitment method. Secondly, we investigated whether its influence remained significant after controlling for experimental condition and baseline differences between the samples. In the second model, therefore, experimental condition and baseline differences between the two groups were included as potential covariates.

To determine whether the two samples differed with regard to quit attempts and 24-hour point prevalence abstinence, logistic regression analyses were conducted. The first model again only consisted of recruitment method, while in the second model experimental condition and baseline differences between the samples were 
included as potential covariates. In a third model, interactions between recruitment method and baseline differences between the two samples were included as well.

\section{RESULTS}

\section{Differences in recruitment rates and respondent characteristics}

Via general practices, 409 eligible smokers were recruited, resulting in €15,32 (€6266,40/409) spent per smoker recruited. Via the mass media approach, 1154 smokers were recruited, resulting in €1,03 (€1190/1154) spent per smoker recruited. Table 4.1 shows the baseline characteristics of the sample recruited via general practices and the sample recruited via mass media, respectively. Respondents recruited via general practices were significantly lower educated and less addicted, and had a higher intention to quit smoking and to maintain non-smoking than their mass media counterparts. The general practice sample consisted of significantly fewer men and of more respondents reporting a cardiovascular or respiratory disease compared with the mass media sample.

Based on these findings, analyses investigating the influence of recruitment method on retention and abstinence rates were controlled not only for experimental condition, but also for gender, educational level, addiction level, intention to quit smoking, intention to maintain non-smoking and for the occurrence of cardiovascular and respiratory diseases. Additionally, with regard to abstinence rates, interaction effects between recruitment method and each of the factors mentioned were studied. 
Table 4.1 Sample characteristics at baseline for the two samples of Dutch adult smokers recruited from May 2009 to June 2010

\begin{tabular}{|c|c|c|c|c|c|}
\hline & $\begin{array}{c}\text { General practice } \\
\text { recruitment }(\mathrm{N}=440)\end{array}$ & $\begin{array}{c}\text { Mass media recruitment } \\
\qquad(\mathrm{N}=1257)\end{array}$ & $t(d f)$ & $\chi^{2}$ & p-value \\
\hline Mean (SD) age & $48.6(12.8)$ & $47.7(31.6)$ & $.55(1691)$ & - & .583 \\
\hline Percent $(\mathrm{N})$ male & $40.2(177)$ & $48.0(603)$ & - & 7.87 & .005 \\
\hline $\begin{array}{l}\text { Percent }(\mathrm{N}) \text { educational } \\
\text { level } \\
\quad \text { High } \\
\quad \text { Medium } \\
\quad \text { Low }\end{array}$ & $\begin{array}{l}23.0(101) \\
44.8(197) \\
32.3(142)\end{array}$ & $\begin{array}{l}32.5(408) \\
46.5(585) \\
21.0(264)\end{array}$ & - & 27.34 & .000 \\
\hline $\begin{array}{l}\text { Percent }(\mathrm{N}) \text { with Dutch } \\
\text { nationality }\end{array}$ & $98.0(431)$ & $97.8(1229)$ & - & .05 & .822 \\
\hline $\begin{array}{l}\text { Percent }(\mathrm{N}) \text { with } \\
\text { cardiovascular diseases }\end{array}$ & $14.3(63)$ & $9.0(113)$ & - & 9.85 & .002 \\
\hline $\begin{array}{l}\text { Percent }(\mathrm{N}) \text { with } \\
\text { respiratory diseases }\end{array}$ & $28.6(126)$ & $14.6(184)$ & - & 42.48 & .000 \\
\hline $\begin{array}{l}\text { Percent }(N) \text { with } \\
\text { diabetes }\end{array}$ & 4.9 & 3.4 & - & .67 & .41 \\
\hline Percent (N) with cancer & 7.1 & 5.4 & - & 1.24 & .27 \\
\hline Percent $(\mathrm{N})$ pregnant & .7 & .5 & - & .10 & .76 \\
\hline Mean (SD) FTND score & $4.2(0.8)$ & $5.0(2.5)$ & $\begin{array}{c}-5.64 \\
(1695)\end{array}$ & - & .000 \\
\hline $\begin{array}{l}\text { Mean (SD) number of } \\
\text { previous quit attempts }\end{array}$ & $3.7(5.8)$ & $4.7(16.0)$ & $\begin{array}{c}-1.28 \\
(1695)\end{array}$ & - & .200 \\
\hline $\begin{array}{l}\text { Mean (SD) intention to } \\
\text { quit smoking }\end{array}$ & $6.3(0.8)$ & $6.2(0.9)$ & $3.92(1625)$ & - & .000 \\
\hline $\begin{array}{l}\text { Mean (SD) intention to } \\
\text { maintain non-smoking }\end{array}$ & $6.1(0.9)$ & $5.9(1.0)$ & $3.40(1625)$ & - & .001 \\
\hline
\end{tabular}

Note: p-values $<.05$ are marked bold

\section{Differences in retention rates}

Of the 1154 eligible respondents recruited via mass media, 601 (47.8\%) were retained at the first (two days after their set quit date) and 454 (36.1\%) at the second (six weeks after baseline) follow-up. For the sample recruited via general practices $(\mathrm{N}=409)$ retention rates were $263(59.8 \%)$ and $210(47.7 \%)$ respectively.

Table 4.2 shows that retention at the first follow-up could significantly be predicted by recruitment method: significantly fewer respondents recruited via mass media than respondents recruited via general practices remained in the sample. However, when controlling for experimental condition and baseline differences between the two samples, the effect of recruitment method became non-significant. The level of addiction, however, appeared to be a significant predictor of retention. That is, respondents who were relatively more addicted to nicotine were less likely to remain in the study than those who were less addicted.

Similar results were found with regard to retention at the second follow-up: significantly fewer respondents remained in the sample when they were recruited 
via mass media than when they were recruited via their general practice. Again, this effect became non-significant when controlling for experimental condition and baseline differences between the samples, while the level of addiction did significantly predict retention. Again, respondents who were more addicted to nicotine were less likely to remain in the study than those who were less addicted. In addition, gender and the occurrence of cardiovascular diseases turned out to be significant predictors of retention at the second follow-up. Female respondents and respondents with a cardiovascular disease significantly more often retained in the study than male respondents and respondents without a cardiovascular disease.

Table 4.2 Predictors of retention at the first (2 days after the set quit date) and second ( 6 weeks) followup in Dutch adult smokers recruited from May 2009 to June 2010 (N=1563)

\begin{tabular}{|c|c|c|c|c|}
\hline & \multicolumn{2}{|c|}{ First follow-up } & \multicolumn{2}{|c|}{ Second follow-up } \\
\hline & OR & $95 \% \mathrm{CI}$ & OR & $95 \% \mathrm{CI}$ \\
\hline Recruitment methoda & $1.62 *$ & $1.28-2.04$ & $1.63^{*}$ & $1.30-2.04$ \\
\hline Recruitment methoda & 1.52 & $.80-2.90$ & 1.10 & $.59-2.04$ \\
\hline Experimental group ${ }^{b}$ & 1.19 & $.94-1.50$ & .99 & $.78-1.25$ \\
\hline $\operatorname{Sex}^{c}$ & .86 & $.68-1.08$ & $.78^{*}$ & $.62-.99$ \\
\hline Medium educational leveld & .98 & $.72-1.34$ & 1.07 & $.78-1.47$ \\
\hline High educational leveld & .72 & $.52-1.01$ & 1.11 & $.79-1.56$ \\
\hline Addiction level & $.93^{*}$ & $.88-.98$ & $.94^{*}$ & $.89-.99$ \\
\hline Intention to quit & 1.12 & $.93-1.34$ & .93 & $.78-1.13$ \\
\hline Intention to maintain non-smoking & .99 & $.84-1.16$ & 1.09 & $.92-1.28$ \\
\hline Cardiovascular diseasese & 1.31 & $.88-1.97$ & $1.80^{*}$ & $1.21-2.69$ \\
\hline Respiratory diseasese & 1.03 & $.74-1.43$ & 1.20 & $.86-1.68$ \\
\hline
\end{tabular}

Note: amass media recruitment is the reference category; bthe control group is the reference category; cfemale respondents are the reference category; ${ }^{d a}$ low educational level is the reference category; ${ }^{\text {not }}$ suffering from the disease is the reference category; ${ }^{*} \mathrm{p}<.05$.

\section{Differences in smoking behaviour: quit attempts and abstinence}

At first follow-up, 169 (64.3\%) respondents within the general practice sample reported having made a quit attempt, while in the mass media sample this percentage was almost $14 \%$ lower $\left(\chi^{2}=13.48, \mathrm{p}<.001\right)$. For every smoker having made a quit attempt, $€ 3,90$ (mass media recruitment) and $€ 37,08$ (general practice recruitment) was spent. Table 4.3 shows that respondents recruited via general practices were more likely to report having made a quit attempt than those recruited via the mass media. However, after controlling for experimental group and baseline differences between the samples this effect became non-significant. Experimental condition, however, was a significant predictor of making a quit attempt: compared to respondents in the control group, respondents in the intervention group were significantly more likely to have made a quit attempt. Addiction level was another significant predictor of quit attempts: respondents who were more addicted to nicotine were less likely to have made a quit attempt than those who were less 
addicted. Furthermore, a higher intention to quit smoking at baseline significantly predicted whether a quit attempt was made.

At second follow-up, 91 (43.3\%) respondents recruited via general practices and 151 (33.1\%) respondents recruited via mass media reported having refrained from smoking during the last 24 hours $\left(\chi^{2}=6.49\right.$, $\left.\mathrm{p}<.001\right)$. For every smoker reporting 24 hour point prevalence abstinence, €68,86 (general practice recruitment) and $€ 7,88$ (mass media recruitment) was spent. Table 4.3 shows that those recruited via general practices were significantly more likely to report being abstinent than those recruited via mass media. However, this effect became non-significant when controlling for experimental group and baseline differences between the samples. Respondents in the intervention group were significantly more likely to report abstinence than respondents in the control group. Moreover, those with a low level of education were more likely to be abstinent than those with a medium level of education. Respondents who were more addicted to nicotine were less likely to be abstinent than those who were less addicted, as were respondents with a respiratory disease compared with those without such a disease.

Interactions between recruitment method and baseline characteristics were investigated, but none were of significant influence on quit attempts or 24-hour point prevalence abstinence ( $\mathrm{p}>$.1) (data not tabulated).

Table 4.3 Predictors of quit attempts and 24-hour point prevalence abstinence (ppa) in Dutch adult smokers recruited from May 2009 to June 2010 (N=1563)

\begin{tabular}{|c|c|c|c|c|}
\hline & \multicolumn{2}{|c|}{ Quit attempts } & \multicolumn{2}{|c|}{ 24-hour ppa } \\
\hline & OR & $95 \% \mathrm{CI}$ & OR & $95 \% \mathrm{CI}$ \\
\hline Recruitment methoda & $1.74^{*}$ & $1.29-2.35$ & $1.55^{*}$ & $1.10-2.16$ \\
\hline Recruitment methoda & 2.22 & $.90-5.46$ & 2.15 & $.82-5.65$ \\
\hline Experimental group b & $1.52^{*}$ & $1.08-2.13$ & $1.97 *$ & $1.31-2.96$ \\
\hline $\operatorname{Sex}^{c}$ & 1.01 & $.72-1.42$ & 1.26 & $.84-1.89$ \\
\hline Medium educational leveld & 1.21 & $.78-1.87$ & $.52^{*}$ & $.30-.91$ \\
\hline High educational leveld & .98 & $.60-1.59$ & .74 & $.41-1.33$ \\
\hline Addiction level & $.91 *$ & $.85-.98$ & $.91^{*}$ & $.83-.99$ \\
\hline Intention to quit & $1.85^{*}$ & $1.40-2.45$ & 1.13 & $.81-1.56$ \\
\hline Intention to maintain non-smoking & 1.07 & $.85-1.35$ & 1.16 & $.88-1.53$ \\
\hline Cardiovascular diseasese & .62 & $.35-1.08$ & .63 & $.32-1.21$ \\
\hline Respiratory diseasese & .87 & $.54-1.40$ & $.49^{*}$ & $.26-.92$ \\
\hline
\end{tabular}

Note: amass media recruitment is the reference category; bthe control group is the reference category; cfemale respondents are the reference category; ${ }^{d}$ low educational level is the reference category; ${ }^{\mathrm{n}}$ not suffering from the disease is the reference category; ${ }^{*} \mathrm{p}<.05$.

\section{DISCUSSION}

\section{Main findings}

The first objective of the present study was to investigate whether smokers recruited for participation in a web-based computer tailored smoking cessation programme using a mass media approach differed from smokers recruited via general practices. Mass media recruitment yielded a significantly larger sample of smokers 
than recruitment via general practices, while less money was spent per respondent recruited. Respondents recruited via mass media were significantly higher educated, more addicted to nicotine, less motivated to quit smoking and to maintain non-smoking, and suffered less from respiratory and cardiovascular diseases than those recruited via general practices. The first two of these findings are in line with a previous study that found that respondents recruited via the web, which is one of several available mass media, were relatively higher educated and more addicted (Etter and Perneger, 2001a). However, in absolute numbers, more smokers with a low level of education were recruited via mass media than via general practices. This appears to be in contradiction with the idea that online and mass media recruitment might not be successful in recruiting smokers with a lower socio-economic status (Dalstra et al., 2002, Marmot, 2005). The general practice may thus be the most suitable gateway for recruiting respondents when the target group consists of smokers with a low socio-economic status in particular, but when one's target group consists of smokers, including but not limited to lower socio-economic status smokers, mass media recruitment might be a better strategy. Etter and Perneger (2001) also found that smokers recruited online were younger and more motivated to quit smoking (Etter and Perneger, 2001a), while we did not find any age differences and respondents recruited via mass media appeared to be less motivated to quit than those recruited via general practices. This latter finding could potentially be explained by selection bias; practice nurses might have invited only those smokers to participate who were, in their opinion, sufficiently motivated to quit. Another difference found between the samples was that more respondents with cardiovascular and respiratory diseases were present amongst those recruited via general practices than amongst those recruited via mass media. An explanation could be that people with such chronic diseases visit their GP more frequently (Schers et al., 2008) than smokers without any noticeable health complaints.

The second objective of our study was to investigate the influence of recruitment strategy on retention rates. Although the number of respondents included using a mass media approach was considerably higher, retention rates were significantly higher in the sample of smokers recruited via general practices. In line with the relatively high rates of attrition found in many web-based interventions (with attrition rates ranging up to 73\%) (Blankers et al., 2010, Eysenbach, 2005, McKay et al., 2008, Shahab and McEwen, 2009, Webb, 2009), only 48\% of the respondents recruited via the mass media could be retained at the first follow-up, while $36 \%$ of them was still in the study at the second follow-up. Within the general practice sample, retention rates were much higher ( $60 \%$ and $48 \%$, respectively). This might be explained by the assumed social bond between smokers and their practice nurse (Shum et al., 2000). As interventions are supposed to have the largest effect on behaviour when fully completed, preventing smokers from dropping out of these programmes is highly relevant. Previously, personal contact with a health professional via e-mail, online or via short message services (SMS) has been shown to be conducive to health behaviour change (Webb et al., 2010) and the involvement of a 
health professional might also be promising in keeping up retention rates. However, the practice nurses' involvement within the general practice sample could not prevent that in both samples respondents who were relatively more addicted were more likely to discontinue their participation in the intervention. The level of addiction has previously been associated with lower success rates (Vangeli et al., 2011, West, 2009). It might thus be that in our study respondents who were relatively more addicted failed to quit smoking and therefore, as is also assumed by the using the recommended intention-to-treat approach (Hughes et al., 2003, Velicer et al., 1992), dropped out of the study.

Thirdly, we investigated the influence of recruitment strategy on success rates. Although less money was spent per smoker making a quit attempt and per smoker reporting to be abstinent within the mass media sample, we showed that respondents recruited via general practices were relatively more likely to report having made a quit attempt and being abstinent from smoking than those recruited via the mass media. As previous research already showed the benefits of a health professionals' involvement in smoking cessation interventions with regard to smoking cessation outcomes (Lancaster and Stead, 2005b, Steptoe et al., 1999, Webb et al., 2010), this may be explained by the involvement of practice nurses in recruiting these smokers. However, in the present study, the effect of recruitment method on quit attempts and abstinence mainly seemed to be caused by the effect of the intervention and by baseline differences between the samples.

\section{Study limitations}

The study presented is subject to several limitations. First of all, smokers recruited via their general practice had a chance to receive a single tailored counselling session by a practice nurse as part of the intervention offered. As only 80 out of more than 8000 Dutch general practices (Giesbers, 2008) participated in our study, it was not possible to provide respondents recruited via mass media with the same opportunity. This difference in intervention components might have influenced who agreed to take part in the study and, as a consequence, may have been partially responsible for the differences found in baseline characteristics between the two samples. Retention and success rates, however, were less likely to be affected by the difference in intervention components as the measurement of both retention and success occurred before counselling sessions took place (i.e. after completion of the six-week follow-up questionnaire). Second, it would have been valuable to include multiplicative functions of the intervention's reach and effect in both samples to determine the intervention's public health impact, as impact=reach $\mathrm{x}$ effect (Abrams et al., 1996, Glasgow et al., 1999, Graham et al., 2008, Velicer and Prochaska, 1999). However, even though we know how many smokers enrolled in the intervention via both recruitment methods, it was unknown how many smokers were invited to participate via each method, thus participation rates ('reach') could not be calculated. 


\section{Conclusions}

The findings presented suggest that different recruitment strategies attract different types of smokers for participation in a web-based smoking cessation intervention. Recruitment via general practices may attract smokers who are more likely to quit smoking (e.g. less addicted, more motivated to quit and more likely to complete the intervention), while recruitment using a mass media approach may ultimately lead to a larger absolute number of smokers quitting smoking, as more smokers can be reached using this strategy, against much lower cost. As a result, the choice for a particular recruitment strategy is expected to influence the potential public health impact of the intervention. The present study suggest that the net impact of the intervention might potentially be higher in the sample recruited via mass media as much more smokers were recruited using this strategy. As, however, retention rates were significantly higher within the general practice sample, future research needs to investigate whether the net impact of the intervention within this sample would also be greater on the long run. 


\section{CHAPTER 5}

\section{Effectiveness of a web-based multiple}

computer-tailored smoking cessation programme: a randomized controlled trial among Dutch adult smokers

Published as: Smit, E.S., de Vries, H., Hoving, C. (2012). The effects of a web-based multiple tailored smoking cessation programme: a randomized controlled trial, Journal of Medical Internet Research, 14(3): e82. 


\section{ABSTRACT}

Background Distributing a multiple computer-tailored smoking cessation intervention through the Internet has several advantages for both provider and receiver. Most important, a large audience of smokers can be reached while a highly individualized and personal form of feedback can be maintained. However, such a smoking cessation programme has yet to be developed and implemented in the Netherlands.

Objective To investigate the effects of a Web-based multiple computer-tailored smoking cessation programme on smoking cessation outcomes in a sample of Dutch adult smokers.

Methods Smokers were recruited from December 2009 to June 2010 by advertising our study in the mass media and on the Internet. Those interested and motivated to quit smoking within 6 months $(\mathrm{N}=1123)$ were randomly assigned to either the experimental $(\mathrm{N}=552)$ or control group $(\mathrm{N}=571)$. Respondents in the experimental group received the fully automated Web-based smoking cessation programme, while respondents in the control group received no intervention. After 6 weeks and after 6 months, we assessed the effect of the intervention on self-reported 24-hour point prevalence abstinence, 7-day point prevalence abstinence and prolonged abstinence using logistic regression analyses.

Results Of the 1123 respondents, 449 (40.0\%) completed the 6-week follow-up questionnaire and 291 (25.9\%) completed the 6-month follow-up questionnaire. We used a negative scenario to replace missing values. That is, we considered respondents lost to follow-up to still be smoking. The computer-tailored programme appeared to have significantly increased 24-hour point prevalence abstinence (odds ratio [OR] 1.85; 95\% confidence interval [CI] 1.30-2.65), 7-day point prevalence abstinence (OR 2.17; 95\% CI 1.44-3.27) and prolonged abstinence (OR 1.99; 95\% CI 1.28-3.09) rates reported after 6 weeks. After 6 months, however, no intervention effects could be identified. Results from complete-case analyses were similar.

Conclusions The results presented suggest that the Web-based computer-tailored smoking cessation programme had a significant effect on abstinence reported after a 6-week period. At the 6-month follow-up, however, no intervention effects could be identified. This might be explained by the replacement of missing values on the primary outcome measures due to attrition using a negative scenario. While results were similar when using a less conservative scenario (i.e. complete-case analyses), the results should still be interpreted with caution. Further research should aim at identifying strategies that will prevent high attrition in the first place and, subsequently to identify the best strategies for dealing with missing data when studies have high attrition rates.

Trial registration Dutch Trial Register NTR1351;

http://www.trialregister.nl/trialreg/admin/rctview.asp?TC=1351 (Archived by WebCite at http://www.webcitation.org/67egSTWrz). 


\section{INTRODUCTION}

Worldwide, the smoking of tobacco is the most preventable cause of illness and premature death (WHO, 2008). Therefore, many interventions have been developed aimed at helping smokers to quit. One strategy that has shown both short- and longterm efficacy in changing smoking behaviour is computer-tailoring (Borland et al., 2004, Lancaster and Stead, 2005c, Noar et al., 2007, Strecher, 1999, Dijkstra et al., 1998a, Hoving, 2007). The content of a computer-tailored intervention is adapted to the specific characteristics of a particular individual. This has been shown to attract and keep an individual's attention (Kreuter et al., 1999, de Vries and Brug, 1999), resulting in more thorough processing of information (Dijkstra, 2005). A single tailored feedback message has already proven to be effective in promoting abstinence from smoking (Te Poel et al., 2009), but when tailored information is provided on multiple occasions the impact of the intervention can be increased even more (Borland et al., 2004, Dijkstra et al., 1998c). In addition, a recent meta-analysis found that dynamically tailored interventions (i.e. iterative assessments and feedback) resulted in greater changes in health behaviour than statically tailored interventions (Krebs et al., 2010). As this effect could not be explained solely by the increased number of overall contacts that dynamic tailoring necessitates (Krebs et al., 2010), a computer-tailoring approach may be warranted that consists not only of multiple feedback moments but also of feedback messages that are iterative: the feedback that respondents receive later on during the intervention should not only concern the respondent's present state, but also refer to the changes respondents have made since their enrolment in the programme.

The Internet has been discovered to be a popular gateway for delivering health behaviour change interventions in general (Griffiths et al., 2006) and computer-tailored and smoking cessation interventions in particular (Lustria et al., 2009, Shahab and McEwen, 2009). Using the Internet to provide such programmes may have several advantages for both provider and receiver: it is highly accessible (Centraal Bureau voor de Statistiek, 2009, Internet World Stats, 2010), it has the potential to reach a large audience at minimal cost and participants can take part at any time that is most convenient to them. Furthermore, the behavioural feedback given in computer-tailoring programmes can still be highly individualized and personal (Dijkstra and De Vries, 1999). Moreover, smokers might not succeed the first time they try to quit (Hughes et al., 2004) and seeking help online relatively anonymously may prevent them from feelings of failure and embarrassment, negative feelings that have been shown to be related to a higher temptation to smoke (Rabois and Haaga, 2003) and poorer abstinence outcomes (Leventhal et al., 2008).

Although a key element of computer-tailoring is that the intervention materials are adapted to specific respondent characteristics, some smokers might benefit more than others from particular smoking cessation interventions. For example, the level of nicotine dependence has previously been suggested to moderate the effectiveness of smoking cessation interventions (Fucito et al., 2010). Moreover, a study 
among nicotine patch users identified several participant characteristics moderating the effectiveness of a Web-based computer-tailored intervention (Strecher et al., 2006). It has therefore been recommended, especially with computer-tailored interventions using new media technologies such as the Internet, to investigate which participant characteristics are associated with effectiveness (Noar et al., 2007).

As Web-based multiple tailored smoking cessation feedback has not yet been offered to the Dutch general public outside scientific studies, our research group developed a Web-based multiple computer-tailored smoking cessation programme and offered Dutch adult smokers the opportunity to participate in this programme. The present study investigated the effectiveness of this programme on smoking cessation outcomes reported after 6 weeks and 6 months. To imitate a natural situation in which smokers who do not participate in a smoking cessation programme do not receive the intervention, the control group did not receive any of the intervention's components. Nevertheless, both the intervention and control group were free to use other smoking cessation aids during the study period. In addition, we investigated whether the effect of the intervention was different for specific subgroups of smokers and whether we could detect a dose-response relationship between the number of feedback messages received and abstinence at the last follow-up.

\section{METHODS}

\section{Intervention}

The Web-based multiple computer-tailored smoking cessation programme was based on a previously developed effective single computer-tailored intervention (Dijkstra et al., 1998a), while the I-Change model served as the theoretical framework (De Vries et al., 2003). While filling out the online baseline questionnaire, all respondents were asked to set a quit date within the next 4 weeks. Respondents in both intervention arms were prompted by e-mail to fill in an online follow-up questionnaire 2 days after their set quit date, and after 6 weeks and 6 months. By clicking on a link provided in this e-mail, respondents could start filling out their next follow-up questionnaire immediately, by logging into the system. One e-mail reminder was sent each time that, 1 week after receiving the first invitation, a respondent had still not filled out the particular questionnaire he or she was invited to complete. While respondents in the control group filled out only the questions, for those in the intervention group questions were directly succeeded by relevant online feedback in order to maintain the respondent's attention and to improve retention rates. Iterative and item-based feedback messages were tailored to several respondent characteristics (De Vries et al., 2003): gender, cognitive variables (attitude, social influence and self-efficacy), intention to quit smoking, goal and relapse prevention strategies (action and coping plans), and smoking behaviour. When the questionnaire was completed, feedback messages were combined into one personalized feedback letter. In addition to being able to read the feedback 
letters on the computer screen, respondents were also sent the feedback letters by e-mail, which allowed for the letters to be printed. The 4-5 page feedback letters respondents received at baseline and after 6 weeks consisted of seven components: (1) introduction, including specific feedback on the respondent's smoking behaviour and on his or her intention to quit smoking or to maintain non-smoking; (2) feedback on the respondent's attitude (perceived advantages [pros] and disadvantages [cons]) toward smoking and quitting smoking; (3) feedback on perceived social influence (not) to smoke; (4) feedback on the respondent's reported selfefficacy to refrain from smoking in specific situations, including suggestions on how to cope with these situations; (5) feedback on the extent to which respondents were planning to undertake specific actions (action plans) while preparing their quit attempt; (6) feedback on how to cope with certain difficult situations (coping plans), including the formulation of personal plans in the shape of if-then statements (van Osch et al., 2008); and (7) ending. As we wanted to minimize the burden of filling out a questionnaire by smokers who had recently quit, the feedback letter that respondents received 2 days after the set quit date consisted of only one page, giving feedback on smoking (cessation) behaviour and relapse prevention strategies. Figure 5.1 shows an example of exemplary items regarding the pros of smoking cessation. Figure 5.2 shows an example of a tailored feedback message.

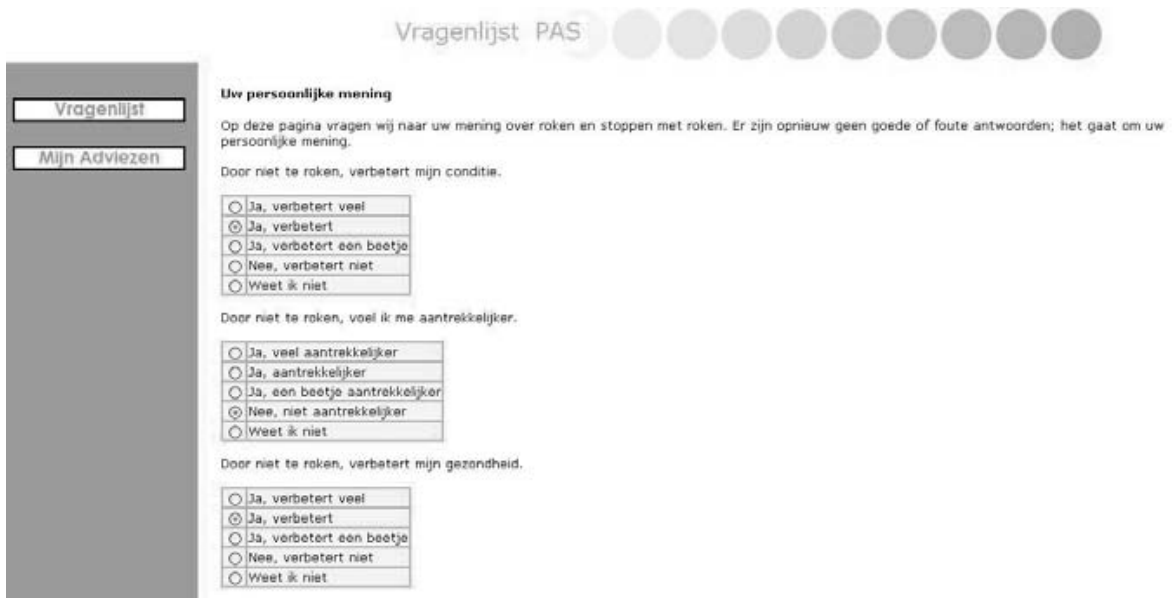

Figure 5.1 Screenshot of items regarding the pros of smoking cessation 


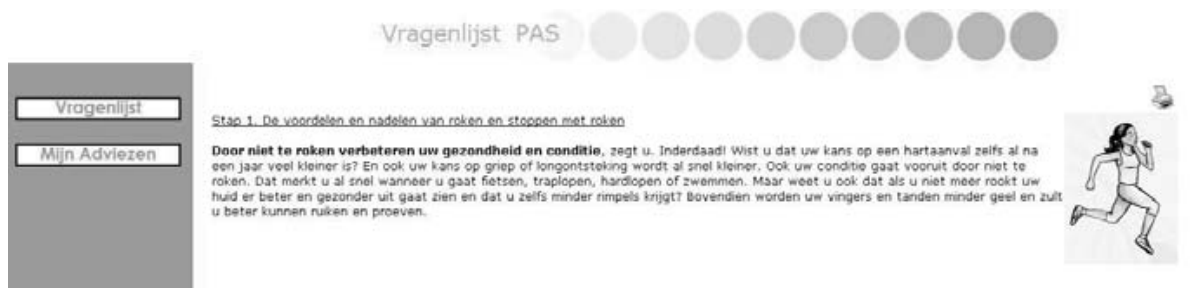

Figure 5.2 Screenshot of personal advice regarding the pros of smoking cessation

All respondents in the experimental condition received at least one tailored feedback letter (i.e. at baseline). At the 6-week follow-up, respondents could have received at most two tailored feedback letters (i.e. at baseline and 2 days after their set quit date), while at the 6-month follow-up, they could have received a maximum of three tailored feedback letters (i.e. at baseline, 2 days after their set quit date and at the 6-week follow-up).

\section{Recruitment and procedure}

The study was approved by the Medical Ethics Committee of Maastricht University and the University Hospital Maastricht (MEC 08-3-037; NL22692.068.08), and is registered with the Dutch Trial Register (NTR1351). A full description of the study protocol is provided elsewhere (Smit et al., 2010).

We recruited adult smokers from December 2009 to June 2010 by advertising our study in the mass media and on the Internet. We sent several press releases to regional newspapers in the Netherlands. Most of these newspapers subsequently mentioned our study on their website, included an item about the project in the print version of their newspaper, or mentioned our study on their local radio station and/or television channel. We also used a Dutch online social network website (Hyves) and several online smoking cessation forums to disseminate our recruitment text. In addition, we advertised our study in a free national newspaper obtainable at all Dutch train stations and several other public places throughout the Netherlands.

We expected after twelve months a $10 \%$ point prevalence abstinence rate in the control condition. Based on results from previous projects, we expected the multiple tailoring programme to lead to a $20 \%$ point prevalence abstinence rate. To be able to detect this difference significantly (alpha=5\%; beta=10\%), according to a 2 -tailed Fisher exact test, we required 281 respondents per arm at the end of the trial (562 respondents in total) (Faul and Erdfelder, 1992). Allowing for $50 \%$ attrition over the trial period, we needed to enrol 1124 respondents at baseline.

Interested smokers could sign up for the study on the study website (http://www.persoonlijkstopadvies.nl) and were eligible to participate if they were 18 years of age or older, were motivated to quit smoking within 6 months and had access to the Internet. On the study website, participants were informed that the study was financed by the Dutch Cancer Society and conducted by researchers from 
Maastricht University in cooperation with the Dutch Expert Center for Tobacco Control (STIVORO). Additionally, the website included information about the objectives of the study, the randomization procedure and the incentive provided when respondents completed all questionnaires, i.e. a €10 voucher. Respondents could choose their own username and password and were informed that no one but the research team was able to retrieve these passwords. As respondents had to report their e-mail address when signing up for the study, we could easily flag respondents with multiple identities and remove them from further analyses. After providing online informed consent, participants were randomly assigned to the intervention group or the control group by a computer software randomization device, allocating approximately $50 \%$ of all respondents to each group. Blinding of respondents was not possible as they had to take notice of whether they were receiving tailored feedback.

\section{Measurements}

All questionnaires used in the present study were previously used and tested among Dutch smoking adults and were self-administered online (Dijkstra et al., 1996, Dijkstra et al., 1998a, Hoving et al., 2010).

\section{Baseline measurement}

We measured six demographic variables: age, gender ( $1=$ male; $2=$ female), educational level (1='low': primary school/basic vocational school; 2='medium': secondary vocational school/high school degree; 3='high': higher vocational school/college degree/university degree), nationality (1=Dutch; $2=$ non-Dutch) and the occurrence of cardiovascular and respiratory diseases (1=no; $2=y e s$ ).

Exclusion criteria were based on current smoking behaviour and motivation to quit smoking: current smoking behaviour was measured by 1 item asking whether the respondent had smoked during the past 7 days (1=no; 2=yes). Motivation to quit smoking was measured by an adapted version of the Stage of Change algorithm (Prochaska and DiClemente, 1983). We asked respondents to state within what time span they intended to quit smoking (1=not within 6 months; $2=$ within 6 months; 3=within 1 month; 4=I have quit, but no longer than 6 months; $5=I$ have quit for longer than 6 months). Respondents who indicated that they had not smoked during the past 7 days, who were not willing to quit within 6 months or who had quit already were excluded from further participation.

We measured overall tobacco consumption using five open-ended questions regarding the number of cigarettes, hand-rolled cigarettes, cigars, cigarillo's and pipes smoked per day. Subsequently, the answers on these five questions were converted into an overall score for tobacco consumption (expressed as number of cigarettes), whereby 1 hand-rolled cigarette or cigarillo equalled 1 cigarette and 1 cigar equalled 4 cigarettes (Mudde et al., 2006). As no concrete guidelines were available for the number of cigarettes that would equal 1 pipe, we conservatively considered 1 pipe to equal 1 cigarette. 
We measured addiction level by the abbreviated Fagerström Test for Nicotine Dependence ( $0=$ not addicted; $10=$ highly addicted) (Heatherton et al., 1991).

We assessed the number of past quit attempts with 1 item, asking the respondents how often they had tried to quit smoking in the past.

\section{Follow-up measurement}

At the 6-week and 6-month follow-ups, we assessed prolonged abstinence by 1 item asking whether the respondent had refrained from smoking since the previous measurement (1=no; $2=y e s$ ). At the 6-week follow-up, prolonged abstinence referred to abstinence since the questionnaire that respondents received 2 days after their set quit date (i.e. at least 2 weeks of abstinence). At the 6-month follow-up, this measure referred to abstinence since 6-week follow-up (i.e. 4.5 months of abstinence). In addition, at both follow-ups we assessed 24-hour and 7-day point prevalence abstinence (ppa), each by 1 item asking whether the respondent had refrained from smoking during the past 24 hours or 7 days (1=no; 2=yes).

\section{Statistical analyses}

First, we conducted descriptive analyses to determine the sample's characteristics. To check for differences between the intervention and control group, we conducted 2-sided t-tests and chi-square tests. Additionally, to determine whether selective drop-out had occurred, we compared those remaining in the study versus those lost to follow-up after 6 weeks and 6 months using 2-sided t-tests and chi-square tests.

Second, we conducted logistic regression analyses to determine whether the intervention had an effect on the outcome measures assessed after follow-up periods of 6 weeks and 6 months. A negative scenario was used to replace missing values. That is, respondents lost to follow-up were considered to still be smoking. To test the robustness of the results, these analyses were also conducted with complete cases only.

Third, to determine whether the effect of the intervention was different for specific subgroups of smokers, we investigated whether we could identify interaction effects between study condition and baseline demographic or behavioural measures using logistic regression analyses.

Data were analyzed using SPSS 17.0 (IBM Corporation, Somers, NY, USA). The significance level used was $\mathrm{P}<.05$.

\section{RESULTS}

\section{Sample characteristics}

Figure 5.3 shows the flow of respondents from enrolment in the study to allocation to the experimental and control condition, retention and whether they were included in the analysis. Of the 1257 respondents assessed for eligibility, 33 (3\%) declined to participate, $32(3 \%)$ were non-smokers at baseline and $69(6 \%)$ were not motivated to quit within six months. Ultimately, 1123 (89.3\%) respondents 
were randomly assigned to either the experimental $(n=552)$ or control $(n=571)$ group and completed the baseline questionnaire. Of the 1123 respondents included, $449(40.0 \%)$ completed the 6 week follow-up questionnaire and 291 (25.9\%) completed the questionnaire at the 6-month follow-up.

Respondents included in the analyses had a mean age of 49.5 years; 535 (47.6\%) were male; and 513 (45.7\%) had a medium level of education. Respondents in the experimental group significantly differed from those in the control condition in their level of education $\left(\chi^{2}=6.11 ; \mathrm{df}=2 ; \mathrm{P}=.047\right)$. Therefore, educational level was included in subsequent analyses as a potential confounder. As no data concerning education level were missing, we included all 1123 respondents in further analyses. Table 5.1 shows characteristics of the overall sample and of the two groups separately.

As table 5.2 shows, no differences were found with regard to baseline characteristics between respondents followed up and respondents lost to follow-up after a 6 week period. After 6 months, however, respondents lost to follow-up were significantly younger $(\mathrm{P}=.02)$ and significantly more addicted $(\mathrm{P}=.01)$ than those who remained in the study.

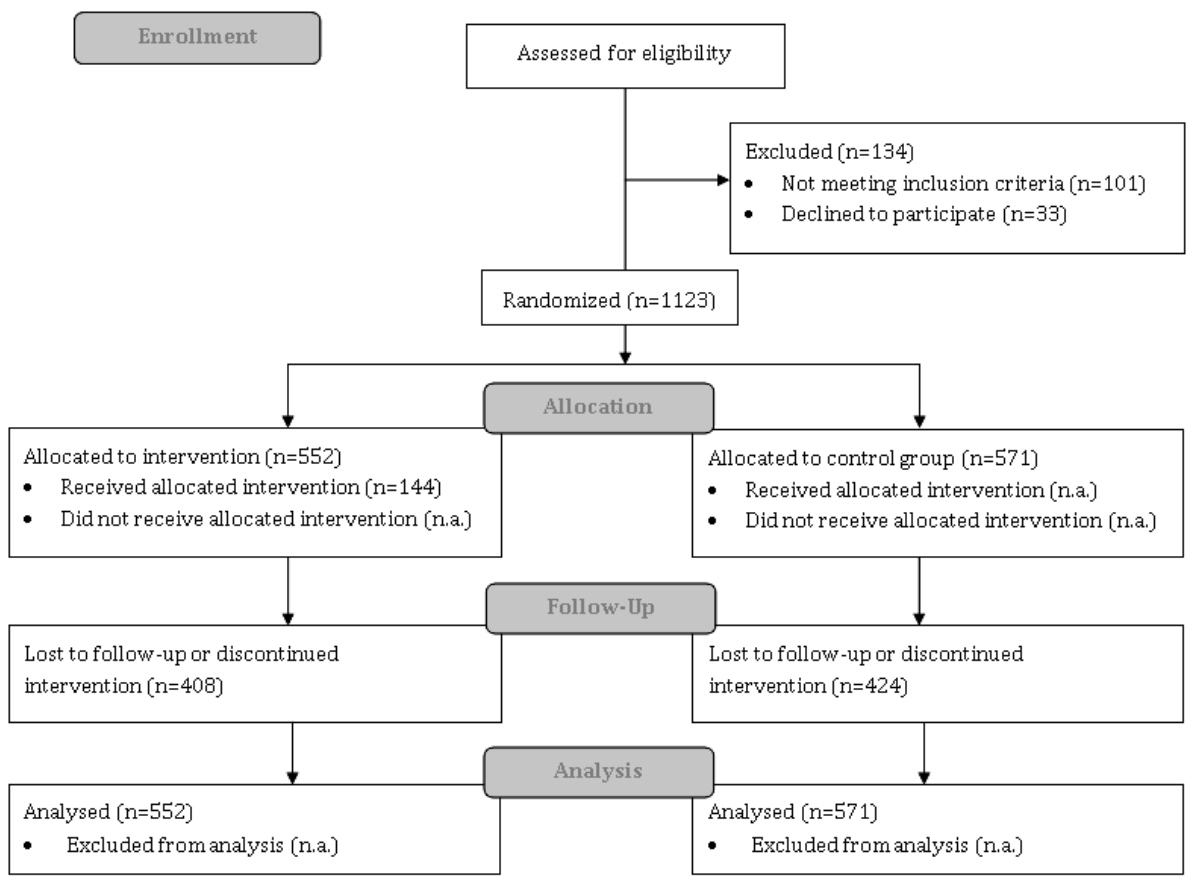

Figure 5.3 Flow of respondents from enrolment in the study to allocation to the experimental and control condition, retention and whether they were included in the analysis 
Table 5.1 Baseline sample characteristics of Dutch smoking adults $(\mathrm{N}=1123)$ recruited from December 2009 up until June 2010

\begin{tabular}{lccc}
\hline Characteristic & $\begin{array}{c}\text { Overall sample } \\
(\mathrm{N}=1123)\end{array}$ & $\begin{array}{c}\text { Experimental group } \\
(\mathrm{n}=552)\end{array}$ & $\begin{array}{c}\text { Control group } \\
(\mathrm{n}=571)\end{array}$ \\
\hline Age (years), mean (SD) & $49.5(32.5)$ & $48.4(12.2)$ & $48.8(12.3)$ \\
Male, \% (n) & $47.6 \%(535)$ & $45 . \%(253)$ & $49.4 \%(282)$ \\
Educational level, \% (n) & & & \\
$\quad$ High & $21.2 \%(238)$ & $19.6 \%(108)$ & $22.8 \%(130)$ \\
$\quad$ Medium & $45.7 \%(513)$ & $43.8 \%(242)$ & $47.5 \%(271)$ \\
$\quad$ Low & $33.1 \%(372)$ & $36.6 \%(202)$ & $29.8 \%(170)$ \\
Dutch, , \% (n) & $97.7 \%(1097)$ & $97.8 \%(540)$ & $97.5 \%(557)$ \\
With cardiovascular diseases, \% (n) & $9.4 \%(106)$ & $11.1 \%(61)$ & $7.9 \%(45)$ \\
With respiratory diseases, \% (n) & $14.3 \%(161)$ & $12.5 \%(69)$ & $16.1 \%(92)$ \\
Number of cigarettes smoked/day, mean (SD) & $20.6(12.4)$ & $20.8(13.7)$ & $20.4(11.0)$ \\
FTNDa score (range 1-10), mean (SD) & $5.1(2.5)$ & $5.0(2.5)$ & $5.2(2.4)$ \\
Number of previous quit attempts, mean (SD) & $5.4(17.5)$ & $5.1(10.1)$ & $5.7(22.4)$ \\
\hline
\end{tabular}

aFagerström Test for Nicotine Dependence.

Table 5.2 A comparison of those followed up and those lost to follow-up after six weeks and six months

\begin{tabular}{lcccc}
\hline & \multicolumn{2}{c}{ 6-week follow-up } & \multicolumn{2}{c}{ 6-months follow-up } \\
Characteristic & $\begin{array}{c}\text { Followed-up } \\
(\mathrm{n}=449)\end{array}$ & $\begin{array}{c}\text { Lost to follow- } \\
\text { up (n=674) }\end{array}$ & $\begin{array}{c}\text { Followed-up } \\
\text { (n=291) }\end{array}$ & $\begin{array}{c}\text { Lost to follow- } \\
\text { up (n=832) }\end{array}$ \\
\hline Age (years), mean (SD) & $50.1(12.2)$ & $49.4(12.6)$ & $50.0(12.2)$ & $48.1(12.3)^{*}$ \\
Male, \% (n) & $44.5 \%(200)$ & $49.7 \%(335)$ & $45.5 \%(133)$ & $48.4 \%(402)$ \\
In experimental condition, \% (n) & $49.9 \%(224)$ & $48.7 \%(328)$ & $49.3 \%(144)$ & $49.1 \%(408)$ \\
Educational level, \% (n) & & & & \\
$\quad$ High & $18.9 \%(85)$ & $22.7 \%(153)$ & $18.5 \%(54)$ & $22.1 \%(184)$ \\
$\quad$ Medium & $45.9 \%(206)$ & $45.5 \%(307)$ & $45.2 \%(132)$ & $45.8 \%(381)$ \\
$\quad$ Low & $35.2 \%(158)$ & $31.8 \%(214)$ & $36.3 \%(106)$ & $32.0 \%(266)$ \\
Dutch, , \% (n) & $98.2 \%(441)$ & $97.3 \%(656)$ & $97.3 \%(284)$ & $97.8 \%(813)$ \\
With cardiovascular diseases, \% (n) & $11.6 \%(52)$ & $8.0 \%(54)$ & $10.6 \%(31)$ & $9.0 \%(75)$ \\
With respiratory diseases, \% (n) & $15.1 \%(68)$ & $13.8 \%(93)$ & $16.8 \%(49)$ & $13.5 \%(112)$ \\
Number of cigarettes smoked/day, mean (SD) & $19.8(12.1)$ & $17.8(6.1)$ & $19.5(11.4)$ & $21.0(12.7)$ \\
FTNDa score (range 1-10), mean (SD) & $4.8(2.3)$ & $4.6(2.3)$ & $4.7(2.3)$ & $5.2(2.5)^{*}$ \\
Number of previous quit attempts, mean (SD) & $5.0(10.6)$ & $5.5(5.9)$ & $5.1(10.0)$ & $5.6(19.5)$ \\
\hline
\end{tabular}

Note: aFagerström Test for Nicotine Dependence; ${ }^{*} \mathrm{P}<.05$.

\section{Effect of the intervention on abstinence}

Of the 552 respondents in the intervention group, 91 (17\%) reported that they had refrained from smoking during the past 24 hours, 74 (13\%) reported that they had not smoked during the past 7 days and 60 (11\%) reported that they had not smoked since the previous measurement 2 days after their quit date. In the control group $(n=571)$ these numbers were $55(10 \%), 38(7 \%)$ and $33(6 \%)$, respectively. 
The intervention had a significant effect on all outcome measures, even when controlling for the baseline difference between the intervention and control groups with regard to their level of education (table 5.3). Significantly more respondents in the intervention group reported having been abstinent for the past 24 hours, the past 7 days or since the previous measurement than respondents in the control group. Results from complete-case analyses were similar (appendix 2).

After 6 months, a total of 51 (9\%) respondents in the intervention group reported having refrained from smoking during the past 24 hours, $45(8 \%)$ reported not having smoked during the past 7 days and $23(4 \%)$ reported not having smoked since the previous measurement. In the control group these numbers were $36(6 \%)$, $34(6 \%)$ and $19(3 \%)$, respectively. Table 5.4 shows that no significant intervention effects were found with regard to all outcome measures reported at 6-month follow-up. The complete-case analyses yielded similar results; these results were slightly more positive regarding 24-hour point prevalence abstinence (appendix 2).

We investigated interaction effects between condition and baseline demographic or behavioural measures, although none of these turned out to have a significant influence on any of the abstinence measures reported after 6 weeks or 6 months (data not reported).

Table 5.3 Effects of the web-based smoking cessation intervention on several behavioural outcomes at six-week follow-up among Dutch adult smokers $(\mathrm{N}=1123)$ recruited from December 2009 up until June 2010

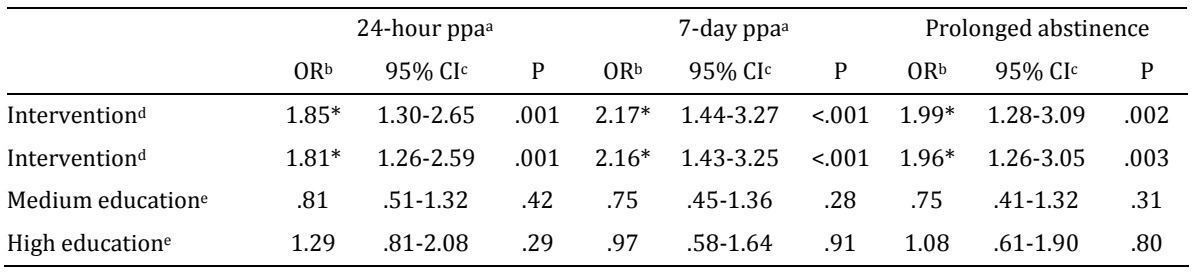

Note: ${ }^{a}$ Point prevalence abstinence; ${ }^{b}$ Odds ratio; ${ }^{c}$ Confidence interval; ${ }^{\mathrm{d} C o n t r o l}$ group is the reference

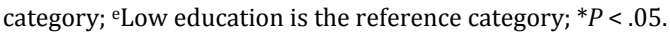

Table 5.4 Effects of the web-based smoking cessation intervention on several behavioural outcomes at six-month follow-up among Dutch adult smokers (N=1123) recruited from December 2009 up until June 2010

\begin{tabular}{lccccccccc}
\hline & \multicolumn{3}{c}{ 24-hour ppa } & \multicolumn{3}{c}{ 7-day ppa } & \multicolumn{3}{c}{ Prolonged abstinence } \\
& $\mathrm{OR}^{\mathrm{b}}$ & $95 \% \mathrm{CI}^{\mathrm{c}}$ & $\mathrm{P}$ & $\mathrm{OR}^{\mathrm{b}}$ & $95 \% \mathrm{Cl}^{\mathrm{c}}$ & $\mathrm{P}$ & OR $^{\mathrm{b}}$ & $95 \% \mathrm{CI}^{\mathrm{c}}$ & $\mathrm{P}$ \\
\hline Intervention $^{\mathrm{d}}$ & 1.51 & $.97-2.35$ & .07 & 1.40 & $.88-2.22$ & .16 & 1.26 & $.68-2.34$ & .46 \\
Intervention $^{\mathrm{d}}$ & 1.47 & $.94-2.30$ & .09 & 1.38 & $.87-2.20$ & .17 & 1.29 & $.69-2.41$ & .42 \\
Medium education $^{\mathrm{e}}$ & .88 & $.48-1.62$ & .69 & .86 & $.47-1.58$ & .62 & .59 & $.28-1.24$ & .16 \\
High education $^{\mathrm{e}}$ & 1.38 & $.76-2.52$ & .29 & 1.10 & $.59-2.05$ & .76 & .56 & $.25-1.26$ & .16 \\
\hline
\end{tabular}

Note: ${ }^{\text {aPoint }}$ prevalence abstinence; ${ }^{\mathrm{b}}$ Odds ratio; ${ }^{\mathrm{c} C o n f i d e n c e}$ interval; ${ }^{\mathrm{d} C o n t r o l}$ group is the reference category; Low education is the reference category. 


\section{DISCUSSION}

\section{Main findings}

In the present study we investigated the effects of a multiple computer-tailored smoking cessation programme delivered through the Internet. The results presented suggest significant effects of the intervention on short-term abstinence: at the 6-week follow-up, respondents who received the intervention were more likely to report being abstinent for the past 24 hours, for the past 7 days and since the previous measurement (i.e. 2 days after their quit date) than those who did not receive the intervention. Despite incorporating goal and relapse prevention strategies (action and coping plans), however, we found no effect of the intervention on abstinence measures assessed after 6 months.

A potential explanation for not finding any suggestion of intervention effects on long-term abstinence might be that more than $70 \%$ of the values on the primary outcome measures had to be replaced, as our study had relatively high levels of attrition, as have many previously developed Web-based interventions (Blankers et al., 2010, Eysenbach, 2005, McKay et al., 2008, Shahab and McEwen, 2009, Webb, 2009, West et al., 2005). In line with the Russell standard (West et al., 2005), we chose a negative scenario to replace missing values (i.e. we considered respondents lost to follow-up to still be smoking). Although this is a recommended analysis when having to deal with drop-out (West et al., 2005), replacing more than $70 \%$ of the values on the primary outcome measures might increase the chances of making a type II error (Field, 2005, Eysenbach, 2005). This may have resulted in an underestimation of the intervention's effectiveness for those who continued to use it (Eysenbach, 2005). On the other hand, analyses with complete cases only are likely to increase the chances of a type I error, which would have resulted in unjustified conclusions in favour of the intervention studied. Although in our study the results from both types of analyses were congruent with each other, the possibilities of errors should be kept in mind when interpreting the results presented. To prevent these problems from occurring in the first place, it is of utmost importance to identify strategies that will ensure the sustained use of Web-based interventions. Previously, several suggestions have been made to prevent attrition, such as ensuring high levels of motivation to quit, providing prompts or reminders, preventing selfcontrol depletion - for example by having respondents form implementation intentions (Webb, 2009) - and providing incentives of at least $€ 10$ (Khadjesari et al., 2011). In addition, a recent review showed that interventions that combined several of these strategies were most effective at facilitating exposure to Web-based interventions (Crutzen et al., 2011). While we took all of the strategies mentioned into account, attrition rates in this study remained high. Evidently, more research is needed to identify strategies that will prevent smokers from dropping out of Webbased behaviour change interventions. Qualitative research among respondents lost to follow-up might further illuminate the main reasons why these respondents to discontinued a Web-based intervention. In a recent study conducted among 
problem drinkers, the most common reasons for not completing a Web-based intervention were personal reasons unrelated to the Web-based intervention, followed by dissatisfaction with the intervention and satisfaction with the improvement in their condition (Postel et al., 2011). Based on the identification of the reasons for discontinuation, novel strategies to prevent attrition need to be developed and tested. In addition, more research is needed to identify strategies for dealing with missing data due to high attrition rates. A recent study comparing six different approaches to missing data concluded that multiple imputation might yield the most valid results (Blankers et al., 2010). However, as the assumption that respondents who drop out should be considered to still be smoking is well-established and a still recommended strategy in smoking cessation research (West et al., 2005), in the present study we opted for this strategy.

Another possible explanation for the lack of intervention effects on long-term abstinence may be that Web-based smoking cessation programmes are not sufficiently tailored and adapted to the long-term wishes of recent ex-smokers to prevent relapse to smoking. Respondents received feedback only at fixed points in time; it was not possible to obtain additional personal feedback or support at times when smokers might have needed it most. The integration of ecological momentary assessment, by collecting real-time data through, for example, palmtops, personal digital assistants or electronic diaries, might be promising. Studies using palmtop computers showed that a decrease in self-efficacy, an increase in positive smoking outcome expectancies and an increase in negative affect predicted the occurrence of a lapse to smoking on the next day (Gwaltney et al., 2005, Shiffman et al., 2007). Integrating ecological momentary assessment into a Web-based intervention might enable us to monitor fluctuations in factors such as self-efficacy and negative affect and, as a consequence, enable us to adapt intervention materials to the needs of recent ex-smokers and, ultimately, to prevent lapses and relapse. In addition, the finding that those lost to follow-up were significantly more addicted to nicotine than those who remained in the study supports the idea that insufficient attention was paid to dealing with withdrawal symptoms. Although in line with current guidelines (Chavannes et al., 2007) we advised smokers who reported smoking more than 10 cigarettes per day to use smoking cessation medication, we did not assess whether these smokers did in fact use such medication during their quit attempt. Even though the Web-based intervention provided information on physical withdrawal symptoms and how to deal with these symptoms, all feedback messages targeted cognitions. As a consequence, solely reading these messages might not have decreased physical withdrawal symptoms. As addiction has been shown to be the most important predictor of a quit attempt's success (McEwen et al., 2008, West, 2009), it may be possible to obtain higher success rates when Web-based smoking cessation interventions are combined with smoking cessation medication aimed at reducing physical withdrawal symptoms. Varenicline, for instance, has been shown to attenuate physical withdrawal symptoms and to prevent relapse to smoking (Hajek et al., 2009, Keating and Siddiqui, 2006). 
We found no support for different intervention effects for specific subgroups of smokers. Based on the results it could thus be argued that the intervention was equally effective for all smokers who participated in the programme. However, respondents who dropped out of the study were relatively more addicted and relatively younger than those who remained in the study, which is in line with previous research (Strecher et al., 2008, Wangberg et al., 2008). A potential explanation might be that younger people have not yet experienced any smoking-related health effects and are, compared with older people who are more often confronted with chronic diseases, less internally motivated to invest time in health behaviour change interventions (de Nooijer et al., 2005).

\section{Study strengths and limitations}

Major strengths of the present study were the large sample of smokers who initiated participation in the smoking cessation programme and the relatively long follow-up period. However, as mentioned previously, the study had relatively high drop-out rates. In the present study, we applied several strategies previously suggested to prevent attrition (Webb, 2009, Khadjesari et al., 2011): using motivation to quit as an inclusion criterion, sending two reminder e-mails for each follow-up questionnaire, encouraging respondents to formulate coping plans in the form of implementation intentions and providing respondents with a €10 voucher for completing all follow-up questionnaires. Despite the actions taken, however, attrition rates remained high. A second limitation is that we could conduct no appropriate dose-response analysis. Insufficient data were available for participants who received one, two, or three letters and who also provided 6-month follow-up data. Of the respondents in the intervention group who provided 6-month follow-up data $(n=144)$, almost $80 \%(n=115)$ received the highest dose of three feedback letters, which resulted in insufficient variation in the doses received to conduct this analysis. As previously stated, we found a significant dose-response relationship between the number of feedback moments and smoking abstinence (Krebs et al., 2010). We therefore recommend that future studies conduct a dose-response analysis to determine whether this effect can be replicated. Finally, we were unable to use continued abstinence as an outcome measure as all respondents were asked to set a quit date within 4 weeks from filling out the baseline questionnaire and were not obliged to quit immediately. According to the Russell standard, however, continued abstinence may classify too many successes as failures due to its strict criteria (West et al., 2005). As we used prolonged abstinence instead, we were still able to assess a long period of abstinence (i.e. at least 4.5 months).

\section{Conclusions}

This Web-based computer-tailored smoking cessation programme had a significant effect on abstinence measured after a 6 week follow-up period. However, this effect had entirely disappeared after 6 months. To prevent relapse, future studies should 
focus on the possibility of applying ecological momentary assessment or combining the present Web-based intervention with the use of smoking cessation medication. Moreover, further research should aim at identifying strategies to prevent smokers from dropping out of Web-based smoking cessation interventions. As completecase analyses and the replacement of missing values using a negative scenario both have their limitations, alternative strategies should be identified and tested (Eysenbach, 2011). 



\section{CHAPTER 6}

Who benefits most? Effectiveness of a web-based multiple computer-tailored smoking cessation programme and tailored counselling by practice nurses on smoking abstinence reported after six and twelve months 


\begin{abstract}
Objective To investigate the effectiveness of an Internet-based multiple computer-tailored smoking cessation programme with (MTC) and without (MT) counselling by a practice nurse compared with usual care (UC) among subgroups of smokers.

Methods A randomized controlled trial with 414 smokers, recruited May 2009-June 2010. Per protocol logistic regression analyses with 24-hour and 7-day point prevalence abstinence (ppa) and prolonged abstinence after six and twelve months as dependent variables.

Results After six months, 157 (38\%) respondents were followed-up; 47\% reported 24-hour ppa, $41 \%$ seven-day ppa and $27 \%$ prolonged abstinence. MT was significantly more effective than UC in increasing 7-day and 24-hour ppa among less addicted smokers and borderline significantly more effective in increasing prolonged abstinence among older smokers. After twelve months, 232 (56\%) respondents were followed-up; 28\% reported 24-hour ppa, 27\% seven-day ppa and $20 \%$ prolonged abstinence. MT was borderline significantly more effective in increasing 24-hour and 7-day ppa among smokers with many previous quit attempts. Conclusions The results presented suggest that the computer-tailoring intervention may be effective in increasing abstinence rates for smokers with many previous quit attempts and for relatively less addicted and older smokers. More research is needed on how to adapt these programmes for other subgroups.
\end{abstract}




\section{INTRODUCTION}

Tobacco smoking is the most preventable cause of illness and premature death worldwide (USDHHS, 2004, WHO, 2008). Computer-tailoring is an effective smoking cessation strategy (Borland et al., 2004, Dijkstra et al., 1998a, Hoving, 2007, Lancaster and Stead, 2005c, Noar et al., 2007, Strecher, 1999, Prochaska et al., 2005). Computer-tailored interventions provide feedback adapted to each smoker's individual characteristics (Dijkstra and De Vries, 1999, Velicer and Prochaska, 1999). Compared with non-tailored interventions, tailored interventions are more successful in attracting and keeping the smoker's attention (Kreuter et al., 1999, de Vries and Brug, 1999, Strecher et al., 2008), resulting in more careful processing of information (Dijkstra, 2005). While a single tailored feedback message is successful in increasing smoking cessation rates (Te Poel et al., 2009), providing feedback multiple times further increases effects (Borland et al., 2004, Dijkstra et al., 1998c). Computer-tailored interventions are increasingly delivered through the Internet (Civljak et al., 2010, Lustria et al., 2009, Shahab and McEwen, 2009), which is highly accessible (Centraal Bureau voor de Statistiek, 2011, Internet World Stats, 2010), can reach a large audience against low costs and offers the opportunity to participate at convenient moments in time (Civljak et al., 2010). An Internet-based multiple computer-tailored intervention would thus be promising. Yet, stand-alone interventions often have limited effects (Lancaster et al., 2000); combining such programmes with other effective interventions might increase effectiveness.

A brief smoking cessation advice from a general practitioner (GP) is such another effective intervention (Lancaster and Stead, 2005a, Stead et al., 2008). GPs, however, often experience a lack of time and skills to support smokers to quit (Yarnall et al., 2003, Hoving et al., 2007b). As nurse-delivered smoking cessation interventions can also be effective (Rice and Stead, 2008, Lemmens et al., 2008) and Dutch GPs often employ a practice nurse (Hingstman and Kenens, 2007), involving practice nurses in smoking cessation counselling can be a viable alternative.

This study investigated the effectiveness of an Internet-based multiple computer-tailored smoking cessation programme with (MTC) and without (MT) counselling by a practice nurse compared with usual care (UC). Additionally, we explored whether effects were different for specific subgroups of smokers, following recent recommendations to determine participant characteristics associated with effectiveness (Noar et al., 2007), such as the level of nicotine dependence (Fucito et al., 2010, Willemsen et al., 1998) and the presence of tobacco-related illnesses (Strecher et al., 2006).

\section{METHODS}

The study was approved by the Medical Ethics Committee of Maastricht University and the University Hospital Maastricht (MEC 08-3-037; NL22692.068.08) and is registered in the Dutch Trial Register (NTR1351). 


\section{Recruitment and procedure}

From May 2009 to June 2010, 91 practice nurses recruited Dutch adult smokers for participation in the study. To aid practice nurses in the recruitment of smoking patients, they were provided with recruitment materials, e.g. desk displays, recruitment letters and posters. Smokers were eligible to participate when they were motivated to quit within six months, were 18 years or older, understood Dutch sufficiently and had Internet access. Interested smokers could sign up on the study website (http://www.persoonlijkstopadvies.nl). After signing an online informed consent form, participants were randomly allocated to receive MTC, MT or UC. Randomization took place at respondent level by means of a computer software randomization device. After randomization, respondents were asked to fill out the baseline questionnaire. After completion of this questionnaire, all respondents were asked to set a quit date within the next four weeks. Based on positive results from previous studies (Dijkstra et al., 1998a, Te Poel et al., 2009), it was hypothesized that questionnaire completion and receipt of tailored feedback (MTC and MT), would result in respondents' increased motivation to quit and a willingness to set a quit date in the near future, a form of action planning previously shown to increase cessation behaviour (Balmford et al., 2010). This reasoning is supported by the high rates of response to this question, ranging from $88 \%$ (MT) to $90 \%$ (MTC) and $91 \%$ (UC). Respondents were prompted by e-mail to fill in follow-up questionnaires two days after their quit date and at six-week, six-month and twelve-month follow-up. When follow-up questionnaires were not completed one week after the invitation, an e-mail reminder was sent, followed-up with a phone call to collect data for the twelve month follow-up.

\section{Questionnaires}

Questionnaires were based on the ICM (De Vries et al., 2003) and tested previously (Dijkstra et al., 1996, Dijkstra et al., 1998a, Hoving et al., 2007a).

\section{Baseline measurements}

Three demographic variables were measured: age, gender and educational level (1='low': primary school/basic vocational school; 2='medium': secondary vocational school/high school degree; 3='high': higher vocational school/college degree/university degree).

Addiction level was measured by the abbreviated Fagerström Test for Nicotine Dependence (FTND) ( $0=$ not addicted; $10=$ highly addicted) (Heatherton et al., 1991).

The number of past quit attempts was assessed with one item, asking respondents how often they had tried to quit smoking in the past.

Depressive symptoms were measured with ten items from the Center for Epidemiologic Studies-Depression scale (CES-D) (Bouma et al., 1995) measured on a 
four-point Likert scale. A mean depression score was calculated $(0=$ no depressive symptoms; 3=many depressive symptoms).

Preparatory plans were assessed by five items, asking whether a respondent planned to undertake perparatory actions for their quit attempt, e.g. to remove all smokers' requisites from the home $(0=$ no; $1=y e s)$. An index was calculated representing the number of action plans made $(0=$ no action plans; $5=$ all action plans).

Coping plans were assessed by nine items, asking whether a respondent wanted to make specific plans to cope with potentially difficult situations, e.g. when taking a break $(0=$ no; $1=y e s)$. An index was calculated representing the number of coping plans made ( $0=$ no coping plans; $9=$ all coping plans).

\section{Outcome measures}

Six and twelve months after baseline, self-reported measures of abstinence were assessed, which was cotinine validated after twelve months using a saliva swap test. Prolonged abstinence was assessed by asking whether the respondent had refrained from smoking since the previous measurement ( $0=$ no; $1=y e s)$. At six-month followup, prolonged abstinence thus referred to abstinence since six-week follow-up (i.e. 4.5 months), while at twelve-month follow-up this measure referred to abstinence since six-month follow-up (i.e. six months). In addition, at both follow-ups 24-hour and 7-day point prevalence abstinence was assessed, by asking whether the respondent had refrained from smoking during the last 24 hours or seven days $(0=$ no; 1 =yes).

\section{The interventions}

Figure 6.1 presented intervention elements for each of the three groups; more details can be found elsewhere (Smit et al., 2010).

\section{Internet-based multiple computer-tailored smoking cessation programme}

Feedback principles were derived from earlier studies (Dijkstra et al., 1998a, Hoving et al., 2010, Te Poel et al., 2009). Feedback messages were tailored to several respondent characteristics based on the I-Change Model (ICM) (De Vries et al., 2003): gender, cognitions (attitude, social influence and self-efficacy), intention to quit, goal and relapse prevention strategies (preparatory and coping plans), and smoking behaviour. Feedback was personalized, descriptive, item-based and iterative or progress comparative (Harrington and Noar, 2012). Feedback letters at baseline, after six weeks and after six months consisted of four to five pages; the feedback letter two days after respondents' quit date consisted of only one page. After questionnaire completion, feedback letters were visible on the computer screen and sent to the respondent by email.

\section{Tailored counselling by practice nurses}

For respondents in the MTC group the tailored feedback letter MT that respondents received at six-week follow-up was replaced by a counselling meeting with a prac- 
tice nurse. After receiving the first tailored feedback letter, MTC respondents were prompted to schedule this meeting within the next six to eight weeks. Practice nurses were provided with a summary of the respondents' situation at six-week follow-up and a counselling protocol to assist them during these sessions. After six months, practice nurses contacted their patients by telephone to ask them about their progress towards permanent cessation and, if needed, to provide additional support.

\section{Usual care}

Respondents in the UC group received smoking cessation guidance according to Dutch standard practice, which can vary from brief stop smoking advice to intensive counselling consisting of four or more sessions (Chavannes et al., 2007, Partnership Stop met Roken, 2009). Practice nurses were instructed to provide UC respondents with cessation support as they were accustomed to provide.

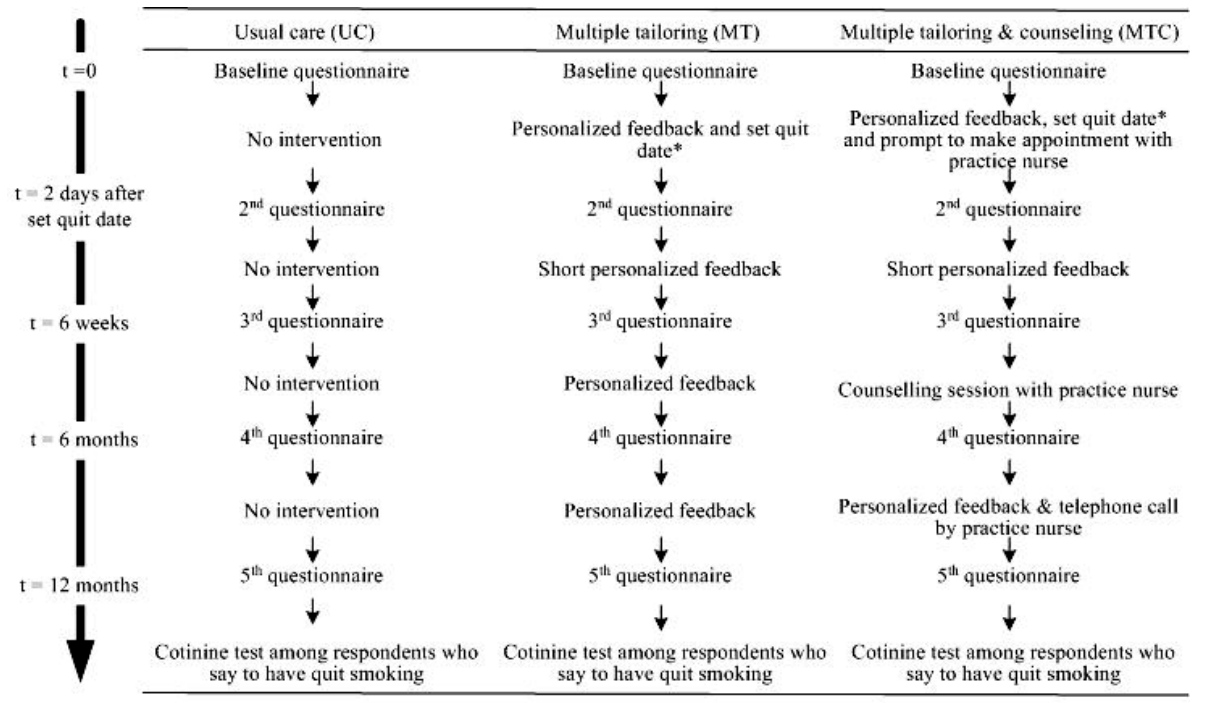

Figure 6.1 Overview of intervention elements received by Dutch smoking adults $(\mathrm{N}=414)$ recruited from May 2009 to June 2010 and randomized into three study groups

\section{Sample size calculation}

Sample size calculations were based on expected seven-day point prevalence abstinence rates at twelve-month follow-up, i.e. $10 \%$ in the control group, $20 \%$ in the MT group and 30\% in the MTC group (Dijkstra et al., 1998a, Hoving et al., 2010). To be able to detect these differences significantly ( $\alpha=5 \% ; \beta=10 \%)$, almost 300 respondents per group were required(DSS Research). Accounting for 20-30\% attrition, 1200 respondents needed to be recruited via 91 practice nurses. 


\section{Statistical analyses}

Sample characteristics were determined using descriptive analyses, while we tested for significant $(\mathrm{p}<.05)$ differences between the three groups using one-way analyses of variance with Tukey's HSD post hoc comparisons, Chi-square tests and, in case of non-normally distributed variables, Kruskal Wallis tests.

As practice nurses work in general practices and respondents were thus nested within these practices we used logistic multilevel regression analyses to determine selective drop-out and the effect of the interventions on primary outcome parameters assessed after six and twelve months. Employing $1^{\text {st }}$ order penalized quasi-likelihood estimation (Rasbash et al., 2009) all available data were analyzed. First, the effects of MTC and MT were compared with the effects of UC, using dummy variables for MTC and MT and UC as the reference category. Secondly, the effects of MTC and MT were compared with each other directly, using dummy variables for MTC and UC and MT as the reference category. A top-down approach was used starting with the most extensive model including main intervention effects, main effects of potential covariates (i.e. age, gender, educational level, addiction level, the number of past quit attempts, depression score, and the number of preparatory and coping plans; based on assumptions from the ICM (De Vries et al., 2003) and findings from previous studies (e.g. Fucito et al., 2010, Strecher et al., 2006), interaction effects between intervention and covariates, a random intercept and random slopes. Firstly, non-significant random effects were removed from the model. Secondly, non-significant interactions effects were removed. Thirdly, nonsignificant covariates were removed. Factors were considered significant when $\mathrm{p}<.10$, using a conservative approach in excluding random and interaction effects (Rosnow and Rosenthal, 1989). When significant interaction effects were detected, subgroup analyses were conducted within subsamples consisting of approximately $50 \%$ of respondents. In subgroup analyses, intervention effects were considered significant when $\mathrm{p}<.05$.

To test the robustness of the results, a sensitivity analysis was conducted regarding all drop-outs as smokers.

Descriptive analyses were conducted with SPSS 15.0. All other analyses were conducted using MLwiN 2.15 (Rasbash et al., 2009).

\section{RESULTS}

\section{Sample characteristics}

In line with the Consolidated Standards of Reporting Trials (CONSORT) (Schulz et al., 2010), figure 6.2 shows the flow of respondents. Of the 445 respondents assessed for eligibility, 9 (2\%) were non-smokers at baseline and 22 (5\%) were not motivated to quit within six months. Ultimately, 414 (93\%) respondents were randomized into the MTC $(\mathrm{N}=163)$, MT $(\mathrm{N}=132)$ or UC $(\mathrm{N}=119)$ group. Respondents had a mean age of 48 years, $166(40 \%)$ of them were male and 187 (45\%) had a 
medium level of education. No significant differences between the three groups were found regarding baseline characteristics (Table 6.1).

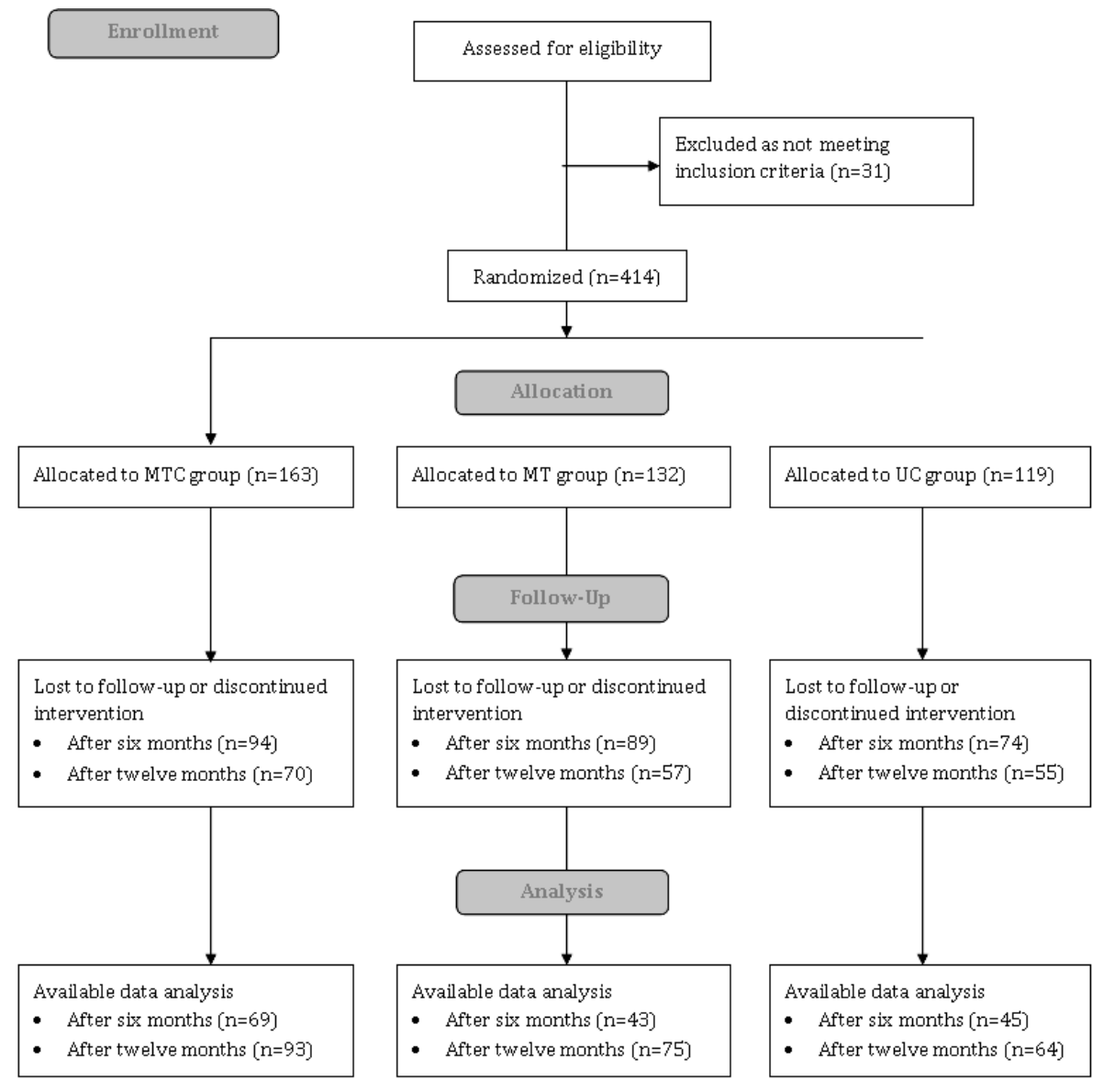

Figure 6.2 CONSORT 2010 Flow Diagram of Dutch smoking adults (N=414) recruited from May 2009 to June 2010 and randomized into three study groups 
Table 6.1 Baseline sample characteristics and abstinence rates at six-month and twelve-month follow-up of Dutch smoking adults recruited from May 2009 to June 2010

\begin{tabular}{|c|c|c|c|c|}
\hline & $\begin{array}{l}\text { Overall sample } \\
\qquad(\mathrm{N}=414)\end{array}$ & $\begin{array}{l}\text { MTC group } \\
(\mathrm{N}=163)\end{array}$ & $\begin{array}{l}\text { MT group } \\
(\mathrm{N}=132)\end{array}$ & $\begin{array}{l}\text { UC group } \\
(\mathrm{N}=119)\end{array}$ \\
\hline Age $^{a}$ & $48.0(11.9)$ & $48.1(12.0)$ & $47.8(12.4)$ & $48.1(11.3)$ \\
\hline Male genderb & $40.1(166)$ & $36.8(60)$ & $41.7(55)$ & $42.9(51)$ \\
\hline \multicolumn{5}{|l|}{ Educational level } \\
\hline High $^{b}$ & $22.7(94)$ & 23.9 (39) & $23.5(31)$ & $20.2(24)$ \\
\hline Mediumb & 45.2 (187) & $41.7(68)$ & $47.7(63)$ & $47.1(56)$ \\
\hline Lowb & 32.1 (133) & $34.4(56)$ & $28.8(38)$ & $32.8(39)$ \\
\hline FTND score $(1-10)^{a}$ & $5.4(2.1)$ & $5.3(2.2)$ & $5.6(2.0)$ & $5.3(2.1)$ \\
\hline $\begin{array}{l}\text { Number of previous quit } \\
\text { attempts }^{\mathrm{c}}\end{array}$ & $3(2-4)$ & $3(2-4)$ & $3(2-4.3)$ & $3(2-5)$ \\
\hline CES-D score $(1-3)^{\mathrm{a}}$ & $.8(.6)$ & $.8(.5)$ & $.9(.7)$ & $.8(.5)$ \\
\hline $\begin{array}{l}\text { Number of preparatory } \\
\text { plans }^{\mathrm{a}}\end{array}$ & $2.9(1.3)$ & $2.9(1.3)$ & $2.9(1.2)$ & $2.8(1.3)$ \\
\hline Number of coping plans ${ }^{a}$ & $4.8(2.7)$ & $4.8(2.6)$ & $5.0(2.8)$ & $4.6(2.8)$ \\
\hline Six-month follow-up & $\begin{array}{l}\text { Overall sample } \\
\qquad(\mathrm{N}=157)\end{array}$ & $\begin{array}{l}\text { MTC group } \\
(\mathrm{N}=69)\end{array}$ & $\begin{array}{l}\text { MT group } \\
(\mathrm{N}=43)\end{array}$ & $\begin{array}{l}\text { UC group } \\
(\mathrm{N}=45)\end{array}$ \\
\hline 24-hour ppa & $46.5(73)$ & $44.9(31)$ & $53.5(23)$ & $42.2(19)$ \\
\hline 7-day ppa & $41.4(65)$ & $36.2(25)$ & $51.2(22)$ & $40.0(18)$ \\
\hline Prolonged abstinence & $27.4(43)$ & $24.6(17)$ & $30.2(13)$ & $28.9(13)$ \\
\hline Twelve-month follow-up & $\begin{array}{l}\text { Overall sample } \\
\qquad(\mathrm{N}=232)\end{array}$ & $\begin{array}{l}\text { MTC group } \\
\quad(\mathrm{N}=93)\end{array}$ & $\begin{array}{l}\text { MT group } \\
(\mathrm{N}=75)\end{array}$ & $\begin{array}{l}\text { UC group } \\
(\mathrm{N}=64)\end{array}$ \\
\hline 24-hour ppa & $27.6(64)$ & $22.6(21)$ & $36.0(27)$ & $25.0(16)$ \\
\hline 7-day ppa & $26.7(62)$ & $21.5(20)$ & $36.0(27)$ & $23.4(15)$ \\
\hline Prolonged abstinence & $19.8(46)$ & $15.1(14)$ & $26.7(20)$ & $18.8(12)$ \\
\hline
\end{tabular}

Note: MTC=Multiple Tailoring and Counselling; MT=Multiple Tailoring; UC=Usual Care; FTND=Fagerström Test for Nicotine Dependence; CES-D=Center for Epidemiologic Studies-Depression scale; ppa=point

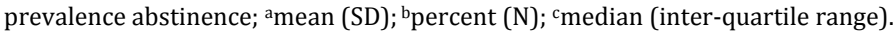

\section{Drop-out analysis}

Of the 414 respondents, 157 (38\%) were followed-up after six and 232 (56\%) after twelve months. Retention after six months was significantly higher among respondents with a high vs. a low level of education (OR 1.97; $\mathrm{p}=.03$ ). Retention after twelve months was significantly predicted by older age (OR 1.03; $p=.01$ ) and experiencing fewer depressive symptoms (OR .63; $\mathrm{p}=.04$ ) (Table 6.2).

\section{Effects of the intervention on smoking abstinence}

After six months, $47 \%$ of respondents reported abstinence for the past 24 hours, $41 \%$ for the past seven days and $27 \%$ reported prolonged abstinence. After twelve months, these percentages were $28 \%, 27 \%$ and $20 \%$, respectively (Table 6.1). Respondents reporting abstinence after twelve months $(\mathrm{N}=64)$ were asked to undergo 
a cotinine test. For 30 respondents (response rate=47\%), validation went successfully and confirmed smoking status; eight respondents refused participation, one test went not successfully and 25 tests were not conducted or not returned to the research team. Our analyses revealed no main effects of the intervention on abstinence assessed after six or twelve months, but showed significant interaction effects with addiction level, age and the number of previous quit attempts, suggesting subgroup analyses.

Table 6.2 Predictors of retention at six-month and twelve-month follow-up among Dutch smoking adults $(\mathrm{N}=414)$ recruited from May 2009 to June 2010

\begin{tabular}{lcccccc}
\hline & \multicolumn{3}{c}{ Six-month follow-up } & \multicolumn{4}{c}{ Twelve-month follow-up } \\
Predictor & OR & $95 \%$ CI & P & OR & $95 \%$ CI & P \\
\hline MTC vs. UC & 1.17 & $.69-1.99$ & .56 & 1.06 & $.62-1.81$ & .83 \\
MT vs. UC & .86 & $.49-1.52$ & .61 & 1.14 & $.64-$ & .67 \\
Age & 1.01 & $.99-1.14$ & .61 & 1.03 & $1.01-1.05$ & .01 \\
Malegender & .99 & $.63-1.56$ & .96 & .93 & $.58-1.48$ & .75 \\
High educational levela & 1.41 & $.85-2.35$ & .19 & 1.56 & $.93-2.59$ & .09 \\
Medium educational levela & 1.97 & $1.08-3.58$ & .03 & 1.35 & $.74-2.47$ & .32 \\
FTND score & 1.03 & $.93-1.14$ & .61 & 1.05 & $.94-1.16$ & .39 \\
Number of previous quit & .98 & $.94-1.03$ & .43 & .99 & $.96-1.03$ & .76 \\
attempts & .72 & $.47-1.11$ & .14 & .63 & $.41-.97$ & .04 \\
CES-D score & 1.01 & $.85-1.20$ & .90 & 1.15 & $.97-1.37$ & .12 \\
Number of preparatory & .96 & $.88-1.04$ & .32 & 1.01 & $.93-1.10$ & .85 \\
plans & Number of coping plans & .96 &
\end{tabular}

Note: MTC=Multiple Tailoring and Counselling; MT=Multiple Tailoring; UC=Usual Care; FTND=Fagerström Test for Nicotine Dependence; CES-D=Center for Epidemiologic Studies-Depression scale; ${ }^{\text {Low }}$ educational level is the reference category.

\section{Interaction effects with addiction level}

A significant interaction effect was found between the type of intervention and addiction level with regard to 24-hour point prevalence abstinence $\left(\chi^{2}=8.63 ; \mathrm{df}=2\right.$; $\mathrm{p}=.04)$ and regarding 7 -day point prevalence abstinence $\left(\chi^{2}=7.73 ; \mathrm{df}=2 ; \mathrm{p}=.02\right)$ at six-month follow-up. Subsequent subgroup analyses among respondents with relatively low (FTND score $\leq 5$ ) and relatively high (FTND score $>5$ ) levels of addiction revealed that MT was significantly more effective than both UC and MTC in increasing 24-hour and seven-day point prevalence abstinence rates among respondents with low levels of addiction (Table 6.3). When drop-outs were regarded as smokers, intervention effects were no longer detected.

\section{Interaction effects with age}

A significant interaction effect was found between the type of intervention and age $\left(\chi^{2}=6.14 ; \mathrm{df}=2 ; \mathrm{p}=.05\right)$ regarding prolonged abstinence assessed after six months. Subgroup analyses among relatively younger (18-48 years) and older (49 years or 
older) respondents revealed that MT was borderline significantly more effective than MTC in increasing prolonged abstinence rates for older respondents (Table 6.4). When drop-outs were regarded as smokers, again no significant differences were found.

Table 6.3 Intervention effects on 24-hour and 7-day point prevalence abstinence assessed after six months - final regression model and subgroup analyses based on addiction level

\begin{tabular}{|c|c|c|c|c|c|c|}
\hline \multirow{2}{*}{ Final regression model } & \multicolumn{3}{|c|}{ 24-hour ppa $(\mathrm{N}=157)$} & \multicolumn{3}{|c|}{ 7-day ppa $(\mathrm{N}=151)$} \\
\hline & $\mathrm{OR}$ & $95 \% \mathrm{CI}$ & $\mathrm{P}$ & OR & $95 \% \mathrm{CI}$ & $\mathrm{P}$ \\
\hline MT vs. UC & 45.54 & $3.55-584.02$ & .003 & 56.26 & $3.90-810.82$ & $<.01$ \\
\hline MTC vs. UC & 6.26 & $.72-54.30$ & .10 & 7.37 & $.64-85.15$ & .11 \\
\hline FTND score & 1.29 & $.97-1.70$ & .08 & 1.42 & $1.02-1.99$ & .04 \\
\hline No. of preparatory plans & 1.41 & $1.07-1.86$ & .02 & 1.59 & $1.16-2.19$ & $<.01$ \\
\hline CES-D score & - & - & - & .40 & $.18-.90$ & .03 \\
\hline Previous quit attempts & - & - & - & 1.08 & $1.00-1.17$ & .04 \\
\hline MTb $x$ FTND score & .54 & $.36-.83$ & .004 & .55 & $.35-.85$ & .01 \\
\hline MTC $^{\mathrm{b}} \mathrm{x}$ FTND score & .72 & $.49-1.04$ & .08 & .67 & $.44-1.03$ & .07 \\
\hline \multicolumn{7}{|l|}{ Subgroup analyses } \\
\hline \multirow{2}{*}{ FTND score 0-5 } & \multicolumn{3}{|c|}{ 24-hour ppaa $(\mathrm{N}=74)$} & \multicolumn{3}{|c|}{ 7-day ppa $(\mathrm{N}=73)$} \\
\hline & OR & $95 \% \mathrm{CI}$ & $\mathrm{P}$ & OR & $95 \% \mathrm{CI}$ & $\mathrm{P}$ \\
\hline MTC vs. MT & .27 & $.07-1.00$ & .05 & .15 & $.04-.66$ & .01 \\
\hline MTC vs. UC & 1.60 & $.54-4.80$ & .40 & 1.63 & $.45-5.95$ & .46 \\
\hline MT vs. UC & 6.02 & $1.43-25.40$ & .01 & 10.60 & $2.04-55.19$ & .01 \\
\hline \multirow{2}{*}{ FTND score 6-10 } & \multicolumn{3}{|c|}{ 24-hour ppaa $(\mathrm{N}=83)$} & \multicolumn{3}{|c|}{ 7-day ppa ${ }^{a}(\mathrm{~N}=78)$} \\
\hline & OR & $95 \% \mathrm{CI}$ & $\mathrm{P}$ & OR & $95 \% \mathrm{CI}$ & $\mathrm{P}$ \\
\hline MTC vs. MT & 1.07 & $.37-3.07$ & .90 & .71 & $.23-2.24$ & .57 \\
\hline MTC vs. UC & .63 & $.20-1.95$ & .42 & .46 & $.13-1.61$ & .22 \\
\hline MT vs. UC & .59 & $.18-1.93$ & .38 & .63 & $.18-2.29$ & .49 \\
\hline
\end{tabular}

Note: Dutch smoking adults were recruited from May 2009 to June 2010; MTC=Multiple Tailoring and Counselling; MT=Multiple Tailoring; UC=Usual Care; FTND=Fagerström Test for Nicotine Dependence; CES-D=Center for Epidemiologic Studies-Depression scale; appa=point prevalence abstinence; ${ }^{\mathrm{b}} \mathrm{UC}$ is the reference category.

\section{Interaction effects with the number of previous quit attempts}

A significant interaction effect was found between the type of intervention and the number of previous quit attempts with regard to 24-hour $\left(\chi^{2}=6.07 ; \mathrm{df}=2 ; \mathrm{p}=.05\right)$ and 7-day point prevalence abstinence $\left(\chi^{2}=4.95 ; \mathrm{df}=2 ; \mathrm{p}=.08\right)$ assessed after twelve months. Subgroup analyses revealed a significant positive effect of MT versus MTC on 24-hour and a borderline significant effect on seven-day point prevalence abstinence among smokers who made five or more previous quit attempts (Table 6.5). Sensitivity analyses revealed similar results. 
Table 6.4 Intervention effects on prolonged abstinence assessed after six months - final regression model and subgroup analyses based on age

\begin{tabular}{|c|c|c|c|}
\hline \multirow{2}{*}{ Final regression model } & \multicolumn{3}{|c|}{ prolonged abstinence $(\mathrm{N}=156)$} \\
\hline & OR & $95 \% \mathrm{CI}$ & $\mathrm{P}$ \\
\hline MT vs. UC & .01 & $.00-.50$ & .02 \\
\hline MTC vs. UC & .13 & $.00-6.32$ & .30 \\
\hline Age & .94 & $.89-1.01$ & .08 \\
\hline No. of preparatory plans & 1.32 & $.96-1.80$ & .09 \\
\hline CES-D score & .46 & $.21-1.04$ & .06 \\
\hline MTa $x$ age & 1.11 & $1.02-1.22$ & .02 \\
\hline MTCa $x$ age & 1.04 & $.96-1.13$ & .35 \\
\hline \multicolumn{4}{|l|}{ Subgroup analyses } \\
\hline \multirow{2}{*}{$18-48$ years of age } & \multicolumn{3}{|c|}{ prolonged abstinence $(\mathrm{N}=72)$} \\
\hline & OR & $95 \% \mathrm{CI}$ & $\mathrm{P}$ \\
\hline MTC vs. MT & 2.64 & $.49-14.34$ & .26 \\
\hline MTC vs. UC & .66 & $.20-2.13$ & .48 \\
\hline MT vs. UC & .25 & $.04-1.42$ & .12 \\
\hline \multirow{2}{*}{$49-76$ years of age } & \multicolumn{3}{|c|}{ prolonged abstinence $(\mathrm{N}=84)$} \\
\hline & OR & $95 \% \mathrm{CI}$ & $\mathrm{P}$ \\
\hline MTC vs. MT & .35 & $.11-1.14$ & .08 \\
\hline MTC vs. UC & 1.06 & $.28-4.07$ & .94 \\
\hline MT vs. UC & 3.05 & $.78-11.84$ & .11 \\
\hline
\end{tabular}

Note: Dutch smoking adults were recruited from May 2009 to June 2010; MTC=Multiple Tailoring and Counselling; MT=Multiple Tailoring; UC=Usual Care; CES-D= Center for Epidemiologic Studies-Depression scale; ${ }^{a} \mathrm{UC}$ is the reference category. 
Table 6.5 Intervention effects on 24-hour and 7-day point prevalence abstinence assessed after twelve months - final regression model and subgroup analyses based on the number of previous quit attempts

\begin{tabular}{|c|c|c|c|c|c|c|}
\hline \multirow{2}{*}{ Final regression model } & \multicolumn{3}{|c|}{ 24-hour ppa $(\mathrm{N}=217)$} & \multicolumn{3}{|c|}{ 7-day ppa ${ }^{\mathrm{a}}(\mathrm{N}=217)$} \\
\hline & OR & $95 \% \mathrm{CI}$ & $P$ & OR & $95 \% \mathrm{CI}$ & $P$ \\
\hline MT vs. UC & .36 & $.12-1.07$ & .07 & .44 & $.16-1.25$ & .12 \\
\hline MTC vs. UC & .88 & $.33-2.37$ & .06 & .98 & $.36-2.65$ & .97 \\
\hline No. of previous quit attempts & 1.00 & $.94-1.06$ & .97 & 1.00 & $.94-1.07$ & .94 \\
\hline No. of preparatory plans & 1.33 & $1.02-1.74$ & .04 & 1.31 & $1.00-1.72$ & .05 \\
\hline $\begin{array}{l}\mathrm{MTC}^{\mathrm{b}} \times \text { no. of previous quit } \\
\text { attempts }\end{array}$ & 1.21 & $1.00-1.46$ & .04 & 1.16 & $.98-1.36$ & .08 \\
\hline $\begin{array}{l}\mathrm{MT}^{\mathrm{b}} \mathrm{x} \text { no. of previous quit } \\
\text { attempts }\end{array}$ & 1.13 & $.98-1.31$ & .10 & 1.13 & $.97-1.30$ & .11 \\
\hline \multicolumn{7}{|l|}{ Subgroup analyses } \\
\hline \multirow{2}{*}{$0-4$ previous quit attempts } & \multicolumn{3}{|c|}{ 24-hour ppaa $(\mathrm{N}=99)$} & \multicolumn{3}{|c|}{ 7-day ppa $(\mathrm{N}=99)$} \\
\hline & OR & $95 \% \mathrm{CI}$ & $\mathrm{P}$ & OR & $95 \% \mathrm{CI}$ & $P$ \\
\hline MTC vs. MT & .72 & $.23-2.23$ & .57 & .72 & $.23-2.23$ & .57 \\
\hline MTC vs. UC & .70 & $.21-2.36$ & .57 & .70 & $.21-2.36$ & .57 \\
\hline MT vs. UC & .97 & $.28-3.43$ & .96 & .97 & $.28-3.43$ & .96 \\
\hline \multirow{2}{*}{ 5-60 previous quit attempts } & \multicolumn{3}{|c|}{ 24-hour ppa ${ }^{a}(\mathrm{~N}=118)$} & \multicolumn{3}{|c|}{7 -day ppa ${ }^{a}(\mathrm{~N}=118)$} \\
\hline & OR & $95 \% \mathrm{CI}$ & $\mathrm{P}$ & OR & $95 \% \mathrm{CI}$ & $P$ \\
\hline MTC vs. MT & .41 & $.16-1.04$ & .06 & .36 & $.14-.95$ & .04 \\
\hline MTC vs. UC & .81 & $.30-2.20$ & .68 & .84 & $.30-2.34$ & .73 \\
\hline MT vs. UC & 2.00 & $.73-5.46$ & .18 & 2.31 & $.83-6.43$ & .11 \\
\hline
\end{tabular}

Note: Dutch smoking adults were recruited from May 2009 to June 2010; MTC=Multiple Tailoring and Counselling; MT=Multiple Tailoring; UC=Usual Care; ${ }^{a}$ ppa=point prevalence abstinence; bUC is the reference category.

\section{DISCUSSION}

\section{Main findings}

The present study suggests that an Internet-based multiple computer-tailored smoking cessation programme may be effective for three subgroups of smokers: those with low levels of addiction, older age and many previous quit attempts. The modest sample size, however, precludes too firm conclusions.

Evidence was greatest for our finding that the computer-tailoring programme was effective in increasing 24-hour and 7-day point prevalence abstinence reported after twelve months among smokers who made relatively many previous quit attempts. Past quitting behaviour is an important predictor of making a quit attempt (Vangeli et al., 2011). It is conceivable that smokers who have previously tried to quit are aware of the situations in which it is difficult for them to remain abstinent and of their personal pitfalls when trying to quit. For them, the mere computertailored programme might have been sufficient in helping them to deal with these difficult situations, while for smokers who had not often tried to quit before it might not have been as they first needed to become aware of their own difficulties in quit- 
ting smoking. More in-depth analysis is needed to assess these potential mechanisms, as also previously recommended (Civljak et al., 2010), and to identify the needs of smokers who have previously not made many quit attempts regarding similar smoking cessation interventions.

The results furthermore suggest that the computer-tailoring programme may be effective in increasing 24-hour and 7-day point prevalence abstinence at sixmonth follow-up for smokers with relatively low addiction levels, something also found by others (McEwen et al., 2008, West, 2009, Willemsen et al., 1998). Nevertheless, even for smokers with low addiction levels no significant intervention effects were detected on prolonged abstinence or abstinence assessed after twelve months, suggesting that even these smokers relapsed to smoking. This might be explained by the fact that respondents only received feedback at fixed points in time; it was not possible to obtain additional feedback or support at times respondents might have needed it most. The integration of ecological momentary assessment, collecting real-time data by e.g. smart phones, might enable us to provide feedback more instantly (Gwaltney et al., 2005, Shiffman et al., 2007) and could possibly be used to further improve the intervention.

Evidence was weakest for our finding that the computer-tailoring programme was effective in increasing prolonged abstinence reported after six months for relatively older smokers. Younger people may not yet experience the detrimental health effects of smoking and might therefore be less internally motivated to invest time in health behaviour change interventions (de Nooijer et al., 2005). This is in accordance with our finding that older respondents significantly more often completed the intervention, which is in line with earlier findings (Strecher et al., 2008). Previously, a significant dose-response relationship was identified between the number of feedback moments and smoking abstinence (Krebs et al., 2010, Strecher et al., 2008). Additionally, a recent systematic review of Internet-based therapy for the treatment of addictions (Gainsbury and Blaszczynski, 2011), such as the smoking of tobacco, suggested a link between respondent engagement and treatment success. Respondents who more actively have used provided interventions, such as websites and e-mail, were more likely to have successful treatment outcomes (Gainsbury and Blaszczynski, 2011). We therefore recommend that future studies investigate how to prevent attrition, especially among younger respondents.

No effects of MTC on abstinence were identified for any subgroups of smokers. Based on previous studies (Curry et al., 1995, Sutton and Gilbert, 2007), we hypothesized that the replacement of one tailored feedback letter by a single, face-toface counselling session with a practice nurse and the addition of a telephone call six months after baseline would significantly add to the effect of the Internet-based programme. Yet, results suggest that this was not the case. A potential explanation might be that one consultation with a practice nurse and the addition of one telephone call were not sufficient to yield an additional effect on smoking abstinence. This explanation is supported by the finding that the effect smoking cessation counselling is weaker when interventions are brief (Rice and Stead, 2008) and might 
suggest that more personal contact with a health professional is needed to result in an increase in effectiveness. Future research should aim to identify the optimal number of face-to-face and/or telephone counselling sessions to be combined with the Internet-based programme. Besides, while the content of the counselling sessions was developed to be as similar as possible to the content of the computertailored feedback letters, it is conceivable that practice nurses' counselling was not congruent with the content and/or style of the computer-tailoring programme. This may suggest that additional training is required for practice nurses on how to attune their counselling to this programme.

Furthermore, no intervention effects could be identified on prolonged abstinence after twelve months, which may be explained by respondents having experienced a lapse (or lapses) to smoking during the intervention period. Although lapses have been shown to strongly predict relapse, not all smokers who lapse ultimately relapse (Ockene et al., 2000, Shiffman, 2005). Some respondents may thus have been abstinent for a prolonged period, but have experienced lapse(s) during the follow-up period. Our definition of prolonged abstinence might have been too strict to identify these smokers as abstinent. In line with the Russell standard (West et al., 2005), we allowed for a grace period until respondents' set quit date, but we did not allow for a maximum of five cigarettes to be smoked as recommended when assessing prolonged abstinence (West et al., 2005). Future studies would benefit from explicitly investigating the occurrence of lapses during the follow-up period and from assessing the exact number of cigarettes smoked during these lapses.

\section{Limitations}

Several limitations were present. First, the study suffered from high rates of attrition. While the drop-out rates found are not extraordinary when compared to other studies (McKay et al., 2008, Shahab and McEwen, 2009, Wangberg et al., 2008), the study was relatively underpowered to conduct some of the (subgroup) analyses, demonstrated by wide confidence intervals and the inability to detect statistically significant intervention effects within certain subgroups of smokers. Nevertheless, the interaction effects found were well interpretable and a larger sample size would probably have resulted in even stronger interaction effects and in stronger intervention effects among subgroups. The results presented should, however, be interpreted with caution. Secondly, usual care for smoking cessation in Dutch general practices varies considerably (Chavannes et al., 2007, Partnership Stop met Roken, 2009). Variation in standard care provided to control groups may substantially influence effect sizes of behaviour change interventions (de Bruin et al., 2009). As anecdotal evidence that we received via interviews with practice nurses, suggests that care as usual in our study was rather intensive, the effects of the intervention on smoking abstinence rates might have been underestimated. 


\section{CONCLUSIONS}

The results presented suggest that an Internet-based multiple computer-tailored smoking cessation intervention may be effective in increasing abstinence rates for smokers with many previous quit attempts and for relatively less addicted and older smokers. More research is needed on how to adapt these programmes for other subgroups smokers. As statistical power was limited, however, future studies should first aim to replicate these findings. 


\section{CHAPTER 7}

The cost-effectiveness and cost-utility of a webbased multiple computer-tailored smoking cessation programme and tailored counselling by practice nurses: a trial-based economic evaluation

Submitted as: Smit, E.S., Evers, S.M.A.A., de Vries, H., Hoving, C. Cost-effectiveness and cost-utility of Internet-based computer-tailoring for smoking cessation. 


\section{ABSTRACT}

Background Although effective smoking cessation interventions exist, information about their cost-effectiveness and cost-utility is thus far limited.

Objective To assess the cost-effectiveness and cost-utility of an Internet-based multiple computer tailored smoking cessation programme and tailored counselling by practice nurses working in Dutch general practices (MTC) compared with the Internet-based multiple computer tailored programme only (MT) and care as usual (UC).

Methods The economic evaluation was embedded in a randomized controlled trial, for which 91 practice nurses recruited 414 eligible smokers. Smokers were randomized to receive MTC $(\mathrm{N}=163)$, MT $(\mathrm{N}=132)$ or UC $(\mathrm{N}=119)$. Self-reported cost and quality of life were assessed during a twelve-month follow-up period. Prolonged abstinence and 24-hour and 7-day point prevalence abstinence were assessed at twelve-month follow-up. The trial-based economic evaluation was conducted from a societal perspective. Uncertainty was accounted for by bootstrapping (1,000 times) and sensitivity analyses.

Results No significant differences were found between the intervention arms with regard to baseline characteristics or effects on abstinence, quality of life and addiction level. However, respondents in the MTC group reported significantly more annual healthcare related costs than respondents in the UC group. Cost-effectiveness analysis, using prolonged abstinence as the outcome measure, showed that MT had the highest probability of being cost-effective. Compared with UC, in this group $€ 5,100$ had to be paid for each additional respondent being abstinent. With regard to cost-utility analyses, using quality of life as the outcome measure, UC was most probably most efficient.

Conclusions To our knowledge, this was the first study to determine the cost-effectiveness and cost-utility of an Internet-based smoking cessation programme with and without counselling by a practice nurse. While the Internet-based multiple computer tailored programme seemed to be the most cost-effective treatment, the cost-utility of care as usual was most probably highest. To ease the interpretation of cost-effectiveness results, however, future research should aim at identifying an acceptable cut-off point for the willingness to pay per abstinent respondent. 


\section{INTRODUCTION}

\section{Background}

Smoking is the single most preventable cause of illness and premature death in the world and is an important risk factor for six of the eight leading causes of death, including several types of cancer, cardiovascular diseases and respiratory diseases (WHO, 2008). Consequently, smoking is related to a reduced quality of life and results in extensive health care costs (Allender et al., 2009, Muennig et al., 2010, Scarborough et al., 2011). Quitting smoking is thus important, not only to improve individual and population health, but also to reduce smoking-related health care costs.

Extensive evidence exists on the clinical effectiveness of behavioural interventions for smoking cessation (Lancaster and Stead, 2005c, Lancaster and Stead, 2005b, Noar et al., 2007). A brief advice from a general practitioner for instance, is one of these effective smoking cessation interventions (Stead et al., 2008). However, general practitioners and practice nurses often report a lack of time and skills to provide their patients with elaborate smoking cessation advice (Hall et al., 2005, Vogt et al., 2005). Computer tailoring is another behavioural intervention proven to be effective in increasing smoking cessation rates up to twelve months (Borland et al., 2004, Dijkstra et al., 1998a, Hoving et al., 2010, Lancaster and Stead, 2005c, Noar et al., 2007, Strecher, 1999, Te Poel et al., 2009). Within a computer tailored intervention, the content of the intervention is adapted to respondents' individual characteristics using computer programmes (de Vries and Brug, 1999). Tailored interventions are more effective in attracting and keeping the smoker's attention (Kreuter et al., 1999, de Vries and Brug, 1999), which results in better processing of information (Dijkstra, 2005). Although a single tailored feedback message is successful in increasing cessation rates (Te Poel et al., 2009), providing feedback on multiple occasions is even more effective (Borland et al., 2004, Dijkstra et al., 1998c). Due to the automatic generation of the tailored feedback and the fact that computer tailored interventions are increasingly delivered online (Lustria et al., 2009, Shahab and McEwen, 2009), the integration of an Internet-based computer tailored programme in the general practice setting might limit the burden on health professionals and could potentially be time-, and thus cost, saving. Our research team has therefore developed a smoking cessation intervention consisting of Internet-based multiple computer-tailoring and a single tailored counselling session by a practice nurse.

Despite their proven clinical effectiveness, information about the relative costeffectiveness of behavioural smoking cessation interventions, e.g. behavioural counselling or eHealth interventions, is limited. Previously, several cost-effective smoking cessation interventions have been developed (Annemans et al., 2009, Hoogendoorn et al., 2010, Vemer et al., 2010). These interventions, however, all involved the use of smoking cessation medication. Intensive counselling combined with pharmacotherapy, for example, yielded low cost per quality adjusted life year 
(QALY) and appeared to be cost-effective compared with care as usual (Hoogendoorn et al., 2010). Furthermore, a recent study in the Netherlands showed that, despite expanding costs due to increased use of smoking cessation support, reimbursement of smoking cessation support would most likely result in more successful quitters and in an increase in QALYs against relatively little cost (Vemer et al., 2010). In addition, the smoking cessation drug varenicline has shown to be associated with both a reduction in smoking-related morbidity and mortality and a decrease in health care costs when compared with bupropion and nicotine replacement therapy (Annemans et al., 2009). With regard to behavioural smoking cessation interventions, one study found that a computer-based smoking cessation intervention aimed at primary care professionals was associated with increases in abstinence rates and QALYs among their patients (Smith et al., 2007). In addition, since there was a call for research to economically evaluate eHealth interventions (Ahern et al., 2006), some studies have been conducted towards the costeffectiveness and cost-utility of Internet-based interventions aimed at reducing alcohol use (Smit et al., 2011) and depressive symptoms (Warmerdam et al., 2010, Gerhards et al., 2010). However, to our current knowledge, no such studies have yet been conducted concerning the cost-effectiveness and cost-utility of an Internetbased smoking cessation intervention.

Therefore, the present study aimed to assess the cost-effectiveness and costutility of an Internet-based multiple computer tailored smoking cessation programme combined with (MTC) and without (MT) a single tailored counselling session by a practice nurses compared with care as usual (UC).

\section{Economic evaluation studies in a nutshell}

In this paragraph, a brief description is provided of each of these steps, in order to provide readers who are less familiar with this type of studies with an overview.

An economic evaluation study aims to determine the costs and effects associated with an intervention and to compare these with other interventions and/or current practice. Results from economic evaluation studies can inform decisions concerning widespread implementation of these interventions, Economic evaluation studies usually consist of five steps (Evers et al., 2010).

The first step consists of identifying relevant costs and effects, looking at the interventions compared and the disease under consideration. This decision is made in line with a chosen perspective, e.g. the health care perspective, health insurer perspective or the societal perspective. However, the government bases its decision to finance and/or implement an intervention on a large scale on all potential societal consequences, both within and outside the care setting. Economic evaluations are therefore preferably conducted from a societal perspective, in which all relevant costs and effects are taken into account (Drummond, 1997).

The second step concerns the measurement of costs and effects. Costs can be assessed prospectively by means of cost diaries or retrospectively using cost questionnaires. Both methods, however, have advantages and disadvantages. Question- 
naires, for instance, are by nature retrospective and are thus subject to recall error. On the other hand, the burden of filling out a prospective cost diary during a relatively long follow-up period could be rather high (Goossens et al., 2000). Effects are usually assessed in terms of quality of life. While both generic and disease-specific quality of life instruments exist, the government wants to compare the costeffectiveness of interventions targeting different diseases. Measuring generic quality of life is therefore usually preferable.

$A$ valuation of the measured costs and effects takes place in the third step. With regard to costs this implies that the number of visits, days and hours are expressed in a monetary currency. To valuate health care and patient costs, manuals for cost analysis in health care research can be used (Hakkaart-van Roijen et al., 2010). These manuals include the standardized average cost price of health care facilities in a particular country. When no standardized cost prices are available, real costs or tariffs can be used to estimate costs. As it is important that all cost prices are comparable, all cost prices should be indexed to the same year. The valuation of effects on quality of life implies that, first, utility scores need to be computed. Utilities refer to preferences that individuals or society may have for a particular set of health outcomes and can range from 0 (the worst imaginable health state) to 1 (perfect health) (Drummond et al., 2005). Subsequently, utility scores assessed at different points in time can be transformed into an overall QALY score, representing the number of QALYs gained or lost during the follow-up period. Gaining one QALY means that one year is gained in perfect health, while e.g. gaining 0.8 QALY means that one year is gained in less than perfect health or that 0.8 years are gained in perfect health.

In the fourth step of the economic evaluation a cost-effectiveness ratio is calculated. In this step, costs and effects are brought together in a ratio. This incremental cost-effectiveness ratio (ICER) can be calculated according to the following formula: ICER $=(\mathrm{Ci}-\mathrm{Cc}) /(\mathrm{Ei}-\mathrm{Ec})$, where $\mathrm{Ci}$ are the adjusted annual costs of the intervention group, Cc the adjusted annual costs of the control group, Ei the adjusted effects for the intervention group and Ec the adjusted effects of the control group. With regard to quality of life, ICERs are often called ICURs: incremental cost-utility ratios. Ratio's such as the ICER or ICUR are, however, only able to compare two groups with each other. An approach which allows us to compared more than two groups with each other when choosing among treatments is to calculate a net monetary benefit (NMB) for each of the treatments. The NMB can be calculated by valuing the effectiveness and utility outcomes in monetary values using a threshold for society's willingness to pay (WTP) per abstinent respondent and per QALY gained (Stinnett and Mullahy, 1998), according to the following formula: (Ei-Ec)*WTP-(Ci-Cc). If the NMB is above 0 , the benefits outweigh the costs and the intervention is considered cost-effective. As many new interventions bring about both additional costs and additional effects, cut-off points have been determined for the WTP per additional measure of effect. In the Netherlands, cut-off points have been established for the WTP per QALY, varying by the severity of the condition (Raad voor de 
Volksgezondheid en Zorg, 2006). For preventive interventions, €18,000 per QALY has been established as an accepted Dutch cut-off point (Raad voor de Volksgezondheid en Zorg, 2006). Similar cut-off points do not yet exist for effects assessed in terms of abstinence rates.

The fifth and last step entails an uncertainty analysis. Each economic evaluation study can suffer from several types of uncertainty. For the present study, two types of uncertainty are most important to explain further. A first type of uncertainty inherent to economic evaluations is sampling uncertainty. Accidentally, respondents can be included in the economic evaluation who reported, for instance, very high health care costs. To deal with this type of uncertainty, bootstrap analyses can be conducted (Drummond et al., 2005, Glick et al., 2007). Using bootstrapping techniques, with replacement, $\mathrm{n}$ (often 1000) times a random sample is drawn from the original dataset, resulting in 1000 slightly different samples and thus slightly different ICERs. Of these 1000 ICERs, the percentage can be calculated with 1) more effects and lower costs (dominant); 2) with less effects and lower costs; 3) with more effects and higher costs and 4) with less effects and higher costs (inferior). In a cost-effectiveness acceptability curve (CEAC), subsequently, the probability of each treatment being most preferable compared with the other treatment(s) studied can be shown for varying levels of the WTP. Secondly, uncertainty results from the fact that for an economic evaluation study it is rather hard to obtain precisely correct data and that certain assumptions have to be made. To test whether results are robust, a sensitivity analysis can be conducted.

For more in-depth information on economic evaluation studies we refer the reader to books about this subject, e.g. Drummond et al. (2005).

\section{METHODS}

\section{Design}

The economic evaluation was embedded in a three-armed randomized controlled trial with a follow-up period of twelve months. The trial design was approved by the Medical Ethics Committee of Maastricht University and the University Hospital Maastricht (MEC 08-3-037; NL22692.068.08), and is registered with the Dutch Trial Register (NTR1351). A more detailed description of the study design has been published elsewhere (Smit et al., 2010).

\section{Participants}

From May 2009 till June 2010, smokers (N=414) were recruited by practice nurses $(\mathrm{N}=91)$ working in Dutch general practices. To aid practice nurses in the recruitment, several recruitment materials were provided (e.g. desk displays, posters and business cards). Respondents were eligible for participation if they smoked, were motivated to quit within six months, were 18 years or older and were able to understand Dutch sufficiently. Moreover, they had to have access to the Internet. Interested smokers could sign up for the study on the study website or through their 
practice nurse. After providing informed consent, participants were randomized into one of the two intervention groups, MTC or MT, or in the UC control group. Randomization took place at respondent level by means of a computer software randomization device.

\section{The smoking cessation intervention}

The smoking cessation intervention consisted of an Internet-based multiple computer tailored smoking cessation programme and tailored counselling by a practice nurse (Smit et al., 2010).

\section{Internet-based multiple tailored smoking cessation programme}

The Internet-based multiple computer tailored smoking cessation programme was based on the I-Change model (De Vries et al., 2003) and on a previously developed, effective single computer tailored intervention (Dijkstra et al., 1998a, Te Poel et al., 2009). Respondents received a total of four feedback letters: at baseline, two days after the quit date they had set at baseline, after six weeks and after six months. Feedback was personalized and tailored to several respondent characteristics: gender, cognitive variables (attitude, social influence and self-efficacy), intention to quit smoking, goal and relapse prevention strategies (action and coping plans), and smoking behaviour. Feedback letters were iterative: the second, third and fourth feedback letters did not only concern the respondent's present state, but also referred to changes respondents had made since they were included in the programme. Respondents were able to access their feedback letters in three ways: it was directly made available online, feedback letters were sent to the respondent by email and they could be printed. An example of a tailored feedback message is provided in appendix 1.

\section{Tailored counselling by practice nurses}

After receiving the first tailored feedback, respondents in the MTC group were prompted to schedule a counselling meeting with their practice nurse within 6 to 8 weeks. They received this counselling session instead of the third tailored feedback letter at six-week follow-up. To assist practice nurses in guiding counselling sessions, they were provided with a counselling protocol. This protocol consisted of three chapters guiding counselling sessions with three different types of respondents: smokers who had quit successfully, smokers who quit but relapsed, and smokers who did not quit yet. The content of the counselling session was developed to be as similar as possible to the content of the computer tailored feedback and was also tailored to the respondents characteristics mentioned above. After six months, practice nurses were instructed to call their patients to ask them about their progress towards permanent cessation and, if needed, to provide them with additional cessation support. 


\section{Usual care}

Respondents randomized in the UC group received smoking cessation guidance according to participating practice nurses' standard practice. Standard practice can vary from a brief intervention consisting of a single stop smoking advice to more intensive interventions consisting of at least four consultations (Chavannes et al., 2007, Partnership Stop met Roken, 2009).

Figure 7.1 presents an overview of the intervention components in each of study groups.

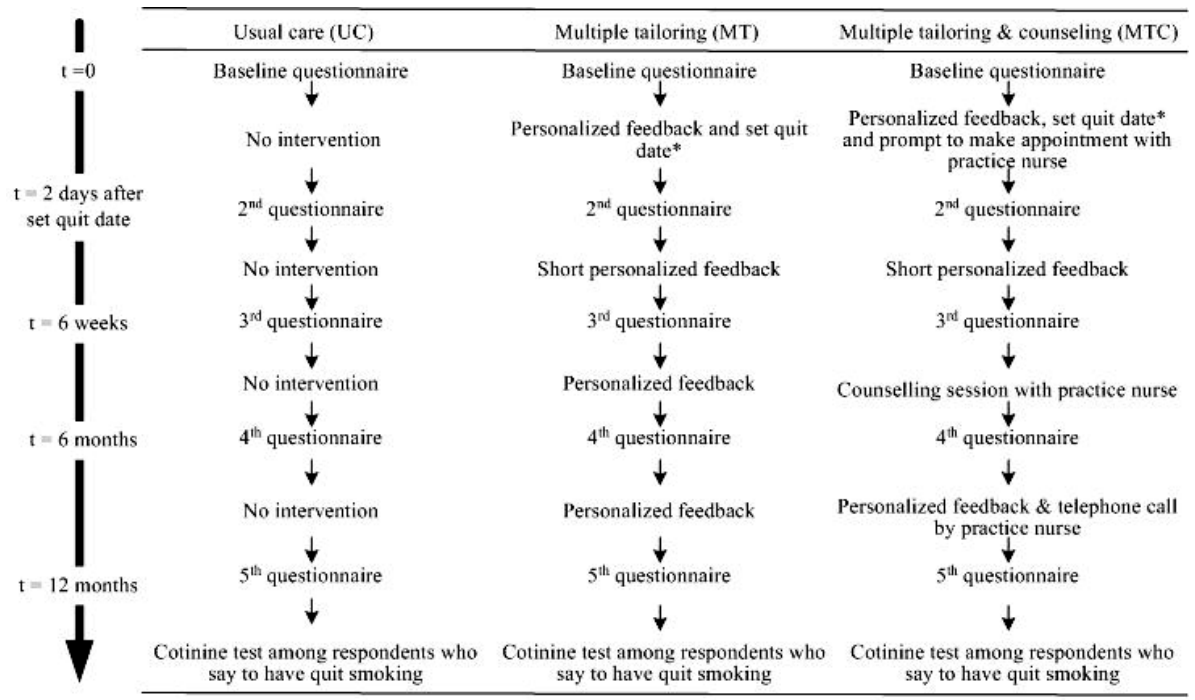

Figure 7.1 Overview of intervention elements received by Dutch smoking adults $(\mathrm{N}=414)$ recruited from May 2009 to June 2010 and randomized into three study groups.

\section{Measurements}

\section{Identification of costs and effects}

The present economic evaluation study was conducted from a societal perspective. This implies that intervention costs, health care costs as well as patient costs were identified as relevant. Intervention costs consisted of all costs that could be attributed to the delivery of the intervention, such as hosting costs for the Internet-based programme and consultation costs. Costs for the development of the intervention as well as research-specific costs were excluded as these costs are sun costs, costs that would not be spend when the intervention would be implemented. Health care costs related to general practitioners' or practice nurses' (telephone) consultations or home visits, inpatient and outpatient specialist care, alternative medicine, mental health care, prescribed and over-the-counter smoking cessation medication, hospital admissions, smoking cessation aids and other care (e.g. paramedics consulta- 
tions or professional home care). Patient costs consisted of travelling and time lost due to participation in the intervention.

\section{Measurement of costs and effects}

Intervention costs, health care costs and patient costs were assessed using a threemonth retrospective costing questionnaire consisting of open-ended questions. Respondents had to indicate whether they had received this type of care during the past three months and if so, how often. These questionnaires were administered at six-week, six-month and twelve-month follow-up. The time respondents spent on the online tailoring programme was tracked by computer registered login and logout data. To assess time spent on counselling, we used a mean time of 20 minutes for face-to-face counselling sessions and an average of 10 minutes for telephone consultations. Travelling time was measured based on average travel distances to a general practitioner in the Netherlands (Hakkaart-van Roijen et al., 2010).

The primary outcome measure used in the cost-effectiveness analysis (CEA) was prolonged abstinence at twelve-month follow-up, assessed by one item asking whether the respondent had refrained from smoking since the previous measurement at six-month follow-up (i.e. abstinence for at least six months) (1=no; 2=yes). Secondary outcome measures were seven day point prevalence abstinence, assessed by one item asking whether the respondent had refrained from smoking during the past seven days (1=no; 2=yes) and addiction level, measured by the abbreviated Fagerström Test for Nicotine Dependence ( $0=$ not addicted; $10=$ highly addicted) (Heatherton et al., 1991). Self-reported abstinence at twelve-month follow-up was cotinine validated using a saliva swap test.

The primary outcomes measure for the cost-utility analysis (CUA) was quality of life, assessed in terms of Quality Adjusted Life Years (QALYs) based on the EuroQol (EQ-5D) (Drummond et al., 2005, EuroQol Group, 1990).

\section{Valuation of costs and effects}

To valuate health care and patient costs, the updated Dutch manual for cost analysis in health care research was used (Hakkaart-van Roijen et al., 2010). In general, standardized cost prices were used, but when no standardized cost prices were available, real costs or tariffs were used to estimate costs. In case of uncertainty, we used the lowest cost price. Costs of smoking cessation medication were calculated based on daily defined dosage (Commissie Farmacotherapautische Hulp CVZ, 2011), including 6\% Value Added Tax, prescription charges for prescribed medication and claw-back, a lawful discount percentage to be subtracted from medication prices by pharmacists (Commissie Farmacotherapautische Hulp CVZ, 2011). Prices of informal care were based on shadow prices for unpaid work. The respondents' time spent on the programme was valued using the friction cost approach (Hakkaart-van Roijen et al., 2010). The index year used was 2011. As cost prices in the Dutch manual for cost analysis in health care research (Hakkaart-van Roijen et al., 2010) were from the year 2009, these cost prices were indexed to the year 2011. 
The consumer price indices used were 105.38 for 2009 and 109.02 for 2011 (Centraal Bureau voor de Statistiek, 2011). A thirteen-month recruitment period and a twelve-month follow-up period can both be considered as relatively short; it is therefore unlikely that any substantial differences in healthcare consumption and effects existed between participants who were included at the beginning and towards the end of the recruitment period. As a result, there were no reasons to discount volumes of healthcare consumption or effects.

QALYs were calculated using respondents' answers to the EQ-5D (Drummond et al., 2005, EuroQol Group, 1990). The EQ-5D consists of five health state dimensions (mobility, self-care, usual activity, pain/discomfort and anxiety/depression) on which respondents have to indicate their own health state $(1=$ no complaints; 2=some complaints; 3=many complaints) (EuroQol Group, 1990). Subsequently, utility scores were computed referring to preferences that individuals or society may have for a particular set of health outcomes (Drummond et al., 2005), based on preferences elicited from a general Dutch population (Lamers et al., 2006). Utilities range from 0 (the worst imaginable health state) to 1 (perfect health) (Drummond et al., 2005). Utility scores assessed at each of the measurement points were used to compute an overall QALY score using the 'area under the curve' method. The area under the curve is the duration of the health state (on the x-axis; twelve months) multiplied by the quality weight for the health state (on the y-axis; utility score), and represents the number of QALYs gained or lost (Drummond et al., 2005).

Smoking abstinence was expressed in a probability score that a smoker would be abstinent ( 1 =not abstinent; $2=$ abstinent).

\section{Analyses}

All analyses were conducted according to the intention-to-treat principle. Missing data for costs, EQ-5D items, overall tobacco consumption and addiction level were replaced by mean imputation, using respondents' scores on the previous and next measurement. When mean imputation was not possible due to missing data on multiple measurement points, missing data were replaced using the last observation carried forward (preferred choice) or next observation carried backward method. Missing data for smoking abstinence were replaced using a negative scenario, whereby respondents lost to follow-up were considered still smoking.

\section{Baseline comparability of the three study groups}

To investigate the comparability of the three groups with regard to demographics, baseline values of outcomes and healthcare-related costs over the last three months, one-way analyses of variance (ANOVA) with Tukey's posthoc tests and Chisquare tests were conducted. To determine whether selective drop-out had occurred, a comparison was made between those lost to follow-up and those who remained in the study after twelve months using two-sided t-tests and Chi-square tests. 


\section{Annual costs and effects}

The three groups were compared with regard to their mean annual costs using nonparametric bootstrapping (5,000 times) with 95\% confidence intervals in percentiles (Drummond et al., 2005). To compare the three groups with regard to the mean effect assessed twelve months after baseline, one-way analyses of variance with Tukey's posthoc tests and Chi-square tests were conducted.

\section{Cost-effectiveness and cost-utility analyses}

First, incremental costs and effects were calculated for each of the three treatments studied. Subsequently, NMBs were calculated enabling us to compare the three groups directly with each other regarding their cost-effectiveness and cost-utility. Using a range of thresholds for the willingness to pay, the likelihood was calculated that each treatment would be most efficient.

\section{Uncertainty analysis}

Sampling uncertainty around the estimates of cost-effectiveness and cost-utility was taken into account using non-parametric bootstrap re-sampling techniques (Drummond et al., 2005, Glick et al., 2007). To deal with the uncertainty of parameter estimates from the primary analyses, a sensitivity analysis was conducted. In primary and secondary analyses patient costs (i.e. traveling and time costs) were not valued in monetary costs but considered as reflected in participants' reported quality of life (Hakkaart-van Roijen et al., 2010). However, as patient costs can be considered directly related to the treatment received (Hakkaart-van Roijen et al., 2010) it could be argued that these costs should be included as part of the programme cost. Therefore, we tested whether an increase in programme costs as a result of patient costs' monetary valuation would lead to a change in results. For the MTC group, the inclusion of patient costs meant an increase in programme costs from $€ 57.70$ to $€ 141.89$ and for the MT group this meant an increase in programme costs from $€ 7.70$ to $€ 82.24$.

Bootstrap analyses were conducted using Microsoft Office Excel 2003. All other analyses were conducted using SPSS 17.0.

\section{RESULTS}

\section{Sample characteristics}

Of the 414 respondents who were eligible for participation, 163 were randomized into the MTC group, 132 into the MT group and 119 into the UC group. No baseline differences were found (Table 7.1). After twelve months, 231 (55.8\%) of the 414 respondents could be followed up. Respondents lost to follow-up were significantly younger $(p=.01)$ than those who remained in the study. Of the 64 respondents who reported to be abstinent after twelve months, 30 did successfully undergo a cotinine test and in all cases self-reported abstinence was confirmed. After imputation of missing values, total cost data were available for 370 respondents (89.2\%), while 
effect data were available for 414 (100\%) (abstinence measures), 409 (98.8\%) (addiction level) and 384 (92.8\%) (QALYs) respondents.

Table 7.1 Comparability of the three groups regarding demographics, baseline values of outcomes and healthcare-related costs over the last three months $(\mathrm{N}=414)$

\begin{tabular}{|c|c|c|c|c|c|c|}
\hline Variable & $\begin{array}{c}\text { MTC } \\
(\mathrm{N}=163)\end{array}$ & $\begin{array}{c}\text { MT } \\
(\mathrm{N}=132)\end{array}$ & $\begin{array}{c}\text { UC } \\
(\mathrm{N}=119)\end{array}$ & $\mathrm{F}$ & $\chi^{2}$ & $P$ \\
\hline Mean (SD) age & $48.1(12.0)$ & $47.8(12.5)$ & $48.1(11.3)$ & .03 & & .97 \\
\hline Percent $(\mathrm{N})$ male & $36.8(60)$ & $41.2(54)$ & $42.9(51)$ & & 1.18 & .56 \\
\hline $\begin{array}{l}\text { Percent (N) educational level } \\
\text { High } \\
\text { Medium } \\
\text { Low }\end{array}$ & $\begin{array}{l}23.9(39) \\
41.7(68) \\
34.4(56)\end{array}$ & $\begin{array}{l}22.9(30) \\
48.1(63) \\
29.0(38)\end{array}$ & $\begin{array}{l}20.2(24) \\
47.1(56) \\
32.8(39)\end{array}$ & & 1.88 & .76 \\
\hline $\begin{array}{l}\text { Percent }(\mathrm{N}) \text { with } \\
\text { cardiovascular diseases }\end{array}$ & $16.6(27)$ & $13.0(17)$ & $15.1(18)$ & & .73 & .69 \\
\hline $\begin{array}{l}\text { Percent }(\mathrm{N}) \text { with respiratory } \\
\text { diseases }\end{array}$ & $23.3(38)$ & $33.6(44)$ & $30.3(36)$ & & 3.99 & .14 \\
\hline Percent $(\mathrm{N})$ with diabetes & $4.9(8)$ & $4.6(6)$ & $5.9(7)$ & & .24 & .89 \\
\hline Percent (N) with cancer & $6.1(10)$ & $9.2(12)$ & $6.7(8)$ & & 1.06 & .59 \\
\hline $\begin{array}{l}\text { Mean (SD) number of } \\
\text { cigarettes smoked per day }\end{array}$ & $20.6(10.3)$ & $23.5(23.2)$ & $21.5(15.5)$ & 1.14 & & .32 \\
\hline $\begin{array}{l}\text { Mean (SD) FTND score (range } \\
1-10 \text { ) }\end{array}$ & $5.3(2.2)$ & $5.6(2.0)$ & $5.3(2.1)$ & .94 & & .39 \\
\hline Mean (SD) utilitya & $0.8(0.2)$ & $0.8(0.2)$ & $0.8(0.2)$ & .60 & & .55 \\
\hline $\begin{array}{l}\text { Mean (SD) healthcare-related } \\
\text { costs }^{\mathrm{b}}\end{array}$ & 425.9 (1506.9) & $286.9(436.6)$ & $236.9(474.0)$ & 1.19 & & .31 \\
\hline GP & $53.2(50.2)$ & $61.0(73.2)$ & $49.7(55.1)$ & 1.11 & & .33 \\
\hline Medical specialist & $65.4(132.1)$ & $78.6(170.9)$ & $87.6(202.8)$ & .57 & & .57 \\
\hline Hospital & 206.9 (1371.3) & $50.9(205.2)$ & $47.9(230.8)$ & 1.47 & & .23 \\
\hline Alternative healer & $5.1(25.1)$ & $9.4(41.6)$ & $4.9(24.2)$ & .84 & & .44 \\
\hline Mental health care & 30.3 (133.5) & $24.3(100.8)$ & $38.2(186.5)$ & .27 & & .76 \\
\hline $\begin{array}{l}\text { Prescribed and OTC } \\
\text { medication }\end{array}$ & $26.7(78.8)$ & $36.4(95.5)$ & $13.6(58.4)$ & 2.37 & & .10 \\
\hline $\begin{array}{l}\text { Medical aids and } \\
\text { assistive devices }\end{array}$ & $1.7(4.1)$ & $3.4(12.6)$ & $1.9(4.8)$ & 1.73 & & .18 \\
\hline Other care & $26.0(268.3)$ & $19.2(166.4)$ & $12.5(65.7)$ & .15 & & .86 \\
\hline
\end{tabular}

aBased on the Dutch algorithm for the Euroqol (EQ-5D) scores; 'bosts prior three months in euro's. 
Table 7.2 Mean annual costs ${ }^{\mathrm{a}}$ per patient and mean effect

\begin{tabular}{|c|c|c|c|c|c|c|c|c|c|}
\hline Costs & MTC & MT & UC & \multicolumn{2}{|c|}{$\begin{array}{c}\text { 95\% CIb Difference } \\
\text { TC- MT }\end{array}$} & \multicolumn{2}{|c|}{$\begin{array}{c}\text { 95\% CI } \text { CIfference }^{\mathrm{b}} \text { Dif - MT } \\
\text { UC }\end{array}$} & \multicolumn{2}{|c|}{$\begin{array}{c}\text { 95\% CI } \text { CI Difference }^{\text {b }} \text { MTC - UC }\end{array}$} \\
\hline $\begin{array}{l}\text { Intervention costs (fixed); } \\
\mathrm{N}=384\end{array}$ & 57.70 & 7.70 & 0.00 & - & - & - & - & - & - \\
\hline $\begin{array}{l}\text { Mean (SD)b healthcare-related } \\
\text { costs; } \mathrm{N}=370\end{array}$ & $\begin{array}{l}1564 \\
(338)\end{array}$ & $1016(158)$ & $761(122)$ & -95.4 & 1381.4 & -642.2 & 139.1 & 194.3 & 1611.8 \\
\hline GP; $N=384$ & $157(14)$ & $180(27)$ & $139(17)$ & -86.1 & 32.3 & -105.8 & 15.5 & -25.4 & 61.4 \\
\hline Medical specialist; $N=374$ & $298(52)$ & $251(62)$ & $224(48)$ & -115.8 & 198.1 & -188.3 & 116.6 & -61.2 & 213.8 \\
\hline Hospital; N=380 & $610(288)$ & $267(106)$ & $172(84)$ & -139.7 & 1054.7 & -374.0 & 161.1 & -17.0 & 1133.4 \\
\hline Alternative healer; $\mathrm{N}=382$ & $17(6)$ & $29(13)$ & $18(9)$ & -42.9 & 13.7 & -43.1 & 18.6 & -23.4 & 18.8 \\
\hline Mental health care; $\mathrm{N}=384$ & $106(39)$ & $95(34)$ & $131(71)$ & -92.2 & 109.8 & -97.1 & 209.4 & -200.2 & 111.9 \\
\hline $\begin{array}{l}\text { Prescribed and OTC smoking } \\
\text { cessation medication; } N=384\end{array}$ & $148(24)$ & $144(30)$ & $90(23)$ & -72.7 & 79.1 & -129.6 & 18.5 & -9.0 & 124.6 \\
\hline $\begin{array}{l}\text { Smoking cessation aids; } \\
\mathrm{N}=384\end{array}$ & $20(10)$ & $15(10)$ & $19(14)$ & -21.6 & 32.5 & -27.9 & 41.0 & -34.4 & 32.3 \\
\hline Other care; $\mathrm{N}=382$ & $122(87)$ & $21(12)$ & $41(22)$ & -15.9 & 293.6 & -24.7 & 72.4 & -45.9 & 281.7 \\
\hline Effects & & & & \multicolumn{2}{|c|}{$\mathrm{F}$} & \multicolumn{2}{|c|}{$\chi^{2}$} & \multicolumn{2}{|c|}{$P$} \\
\hline $\begin{array}{l}\text { Percent }(\mathrm{N}) \text { prolonged } \\
\text { abstinent; } \mathrm{N}=414\end{array}$ & $8.6(14)$ & $15.2(20)$ & $10.1(12)$ & & & \multicolumn{2}{|c|}{3.36} & \multicolumn{2}{|c|}{.19} \\
\hline $\begin{array}{l}\text { Mean (SD) QALY-EQ5D }{ }^{c} \\
\mathrm{~N}=384\end{array}$ & $.86(.15)$ & $.83(.21)$ & $.84(.21)$ & \multicolumn{2}{|c|}{.89} & & \multicolumn{2}{|c|}{.41} \\
\hline $\begin{array}{l}\text { Percent (N) } 7 \text { days abstinent; } \\
N=414\end{array}$ & $12.3(20)$ & 20.5 (27) & $12.6(15)$ & & & & & \multicolumn{2}{|c|}{.10} \\
\hline $\begin{array}{l}\text { Mean (SD) FTND scored; } \\
\mathrm{N}=409\end{array}$ & $4.76(2.41)$ & $5.21(2.30)$ & $4.81(2.46)$ & \multicolumn{2}{|c|}{1.40} & & & \multicolumn{2}{|c|}{.25} \\
\hline
\end{tabular}

aVolumes and cost price details are available upon request; bUnadjusted bootstrapped mean, standard deviation and $95 \%$ confidence interval (CI) based on 5000 bootstrap replications; 'Based on the Dutch

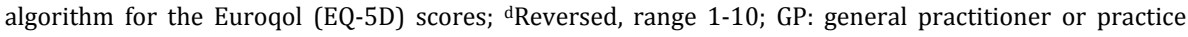
nurses; OTC: over-the-counter.

\section{Annual costs and effects}

A significant difference was found between the MTC and UC group with regard to annual healthcare-related costs per patient (Table 7.2): in the MTC group, mean annual costs were $€ 1,564$ ( $S D=€ 338$ ), while costs in the UC group were $€ 761$ $(\mathrm{SD}=€ 122)$. No differences were found between the three groups with regard to any specific type of healthcare-related costs, nor regarding effects on abstinence, QALYs or addiction level assessed at twelve-month follow-up.

\section{Cost-effectiveness analyses}

Table 7.3 shows that, compared with UC, €5,100 has to be paid in the MT group for each additional respondent being abstinent. For respondents in the MTC group 
costs were higher, while effects were lower than in the UC and MT groups, As a result, MTC was dominated by the other two treatments.

The CEA showed that until a threshold value for the WTP of €5,100 per abstinent respondent, UC was most probably the most efficient treatment. From a WTP of $€ 5,100$ or higher, however, MT was most probably most cost-effective (Table 7.3). With the accepted Dutch cut-off point of €18,000 per QALY for preventive interventions (Raad voor de Volksgezondheid en Zorg, 2006), MT would thus be the most preferable treatment. These results are visually displayed in the costeffectiveness acceptability curve (CEAC), showing the probability of each treatment being preferable to the other two treatments for varying levels of the WTP per additional abstinent participant (Figure 7.2). Sensitivity analyses supported these results (Table 7.3).

Results from secondary analyses showed that concerning seven-day point prevalence abstinence, a high probability was found (i.e. 88\%, with a WTP of $€ 18,000$ per abstinent respondent) that MT was the most cost-effective treatment. Regarding the level of addiction, however, it was most probable that MT would be least efficient (Table 7.4).

\section{Cost-utility analyses}

With regard to QALYs gained, results were somewhat different. As is shown in table 7.3, MT was dominated, as this treatment was both more expensive and less effective than UC. MTC was more expensive, but also more successful than UC and MT, which resulted in an incremental cost of €40,300 per QALY gained when comparing MTC with UC. Comparing MTC with MT resulted in an incremental cost of €18,367 per QALY.

The CUA showed that, with a WTP of €18,000 per abstinent respondent, UC would probably (i.e. 64\%) be the most efficient treatment (Table 7.4). While decreasing this threshold value to $€ 0$ led to an increased probability that UC would be most efficient, increasing this threshold led to a lower probability of UC being most preferable. With a WTP of almost €40,000, UC and MTC would be equally preferable. These results are further illustrated in the cost-utility acceptability curve (CUAC) (Figure 7.3). Sensitivity analyses showed similar results (Table 7.4). 


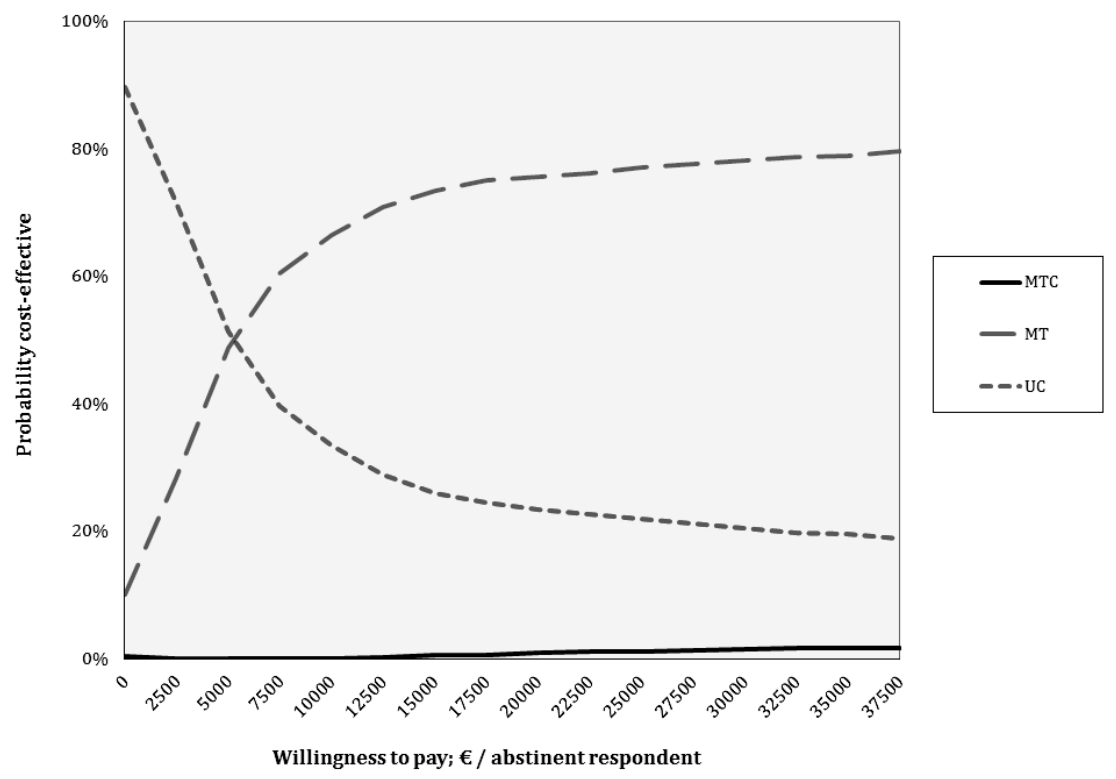

Figure 7.2 Cost-effectiveness acceptability curve

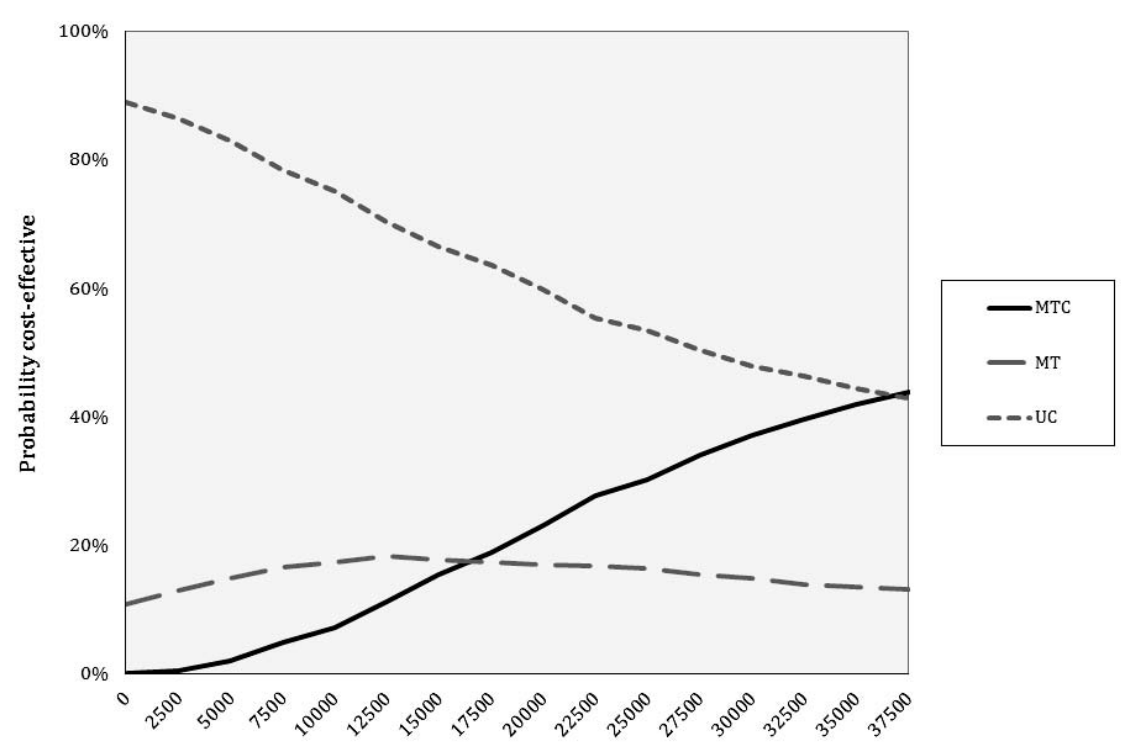

Willingness to pay; € / QALY

Figure 7.3 Cost-utility acceptability curve 
Table 7.3 Incremental costs and effects per abstinent smoker and per QALY gained for the three treatments studied ${ }^{a}$

\begin{tabular}{|c|c|c|c|c|c|}
\hline \multicolumn{6}{|c|}{ Prolonged abstinence $^{c}$} \\
\hline Intervention & $\begin{array}{l}\text { Costs per } \\
\text { participant }\end{array}$ & $\begin{array}{l}\text { Probability } \\
\text { abstinent }\end{array}$ & $\begin{array}{l}\text { Incremental } \\
\text { costs }^{\mathrm{b}}\end{array}$ & $\begin{array}{l}\text { Incremental } \\
\text { probability } \\
\text { abstinent }\end{array}$ & $\begin{array}{l}\text { Incremental costs per } \\
\text { abstinent respondent }{ }^{b, e}\end{array}$ \\
\hline UC & 761 & 1.12 & - & - & - \\
\hline MT vs. UC & 1,016 & 1.17 & 255 & .05 & 5,100 \\
\hline MTC vs. UC & 1,567 & 1.10 & 806 & -.02 & dominated \\
\hline MTC vs. MT & 1,567 & 1.10 & 551 & -.07 & dominated \\
\hline \multicolumn{6}{|l|}{ QALY-EQ5Dd } \\
\hline Intervention & $\begin{array}{l}\text { Costs per } \\
\text { participant }\end{array}$ & Quality of life & $\begin{array}{l}\text { Incremental } \\
\text { costs }^{\text {b }}\end{array}$ & $\begin{array}{l}\text { Incremental } \\
\text { quality of life }\end{array}$ & $\begin{array}{l}\text { Incremental costs per } \\
\mathrm{QALYb,e}\end{array}$ \\
\hline UC & 761 & .84 & - & - & - \\
\hline MT vs. UC & 1,016 & .83 & 255 & -.01 & dominated \\
\hline MTC vs. UC & 1,567 & .86 & 806 & .02 & 40,300 \\
\hline MTC vs. MT & 1,567 & .86 & 551 & .03 & 18,367 \\
\hline
\end{tabular}

aWith a willingness-to-pay threshold of $€ 18,000$; 'In euros; 'Coded as $1=$ not abstinent and $2=$ abstinent; ${ }^{\mathrm{d} B a s e d}$ on the Dutch algorithm for the Euroqol (EQ-5D) scores; ${ }^{\mathrm{e}}$ calculated according to the formula ICER $=(\mathrm{Ci}-\mathrm{Cc}) /(\mathrm{Ei}-\mathrm{Ec})$.

Table 7.4 Results from cost-effectiveness and cost-utility analyses ${ }^{\mathrm{a}}$

\begin{tabular}{|c|c|c|c|c|c|c|}
\hline & \multicolumn{3}{|l|}{$\mathrm{N}$} & \multicolumn{3}{|c|}{ Probability of NB highest $\mathrm{b} ; \%$} \\
\hline & MTC & MT & $\mathrm{UC}$ & MTC & MT & UC \\
\hline \multicolumn{7}{|l|}{ Primary analysis } \\
\hline Prolonged abstinence & 145 & 121 & 104 & 0 & 78 & 21 \\
\hline QALY-EQ5Dc & 145 & 121 & 104 & 18 & 18 & 64 \\
\hline \multicolumn{7}{|l|}{ Secondary analysis } \\
\hline 7 day ppa & 145 & 121 & 104 & 1 & 88 & 11 \\
\hline FTND scored & 135 & 115 & 96 & 50 & 6 & 45 \\
\hline \multicolumn{7}{|l|}{ Sensitivity analysise } \\
\hline Prolonged abstinence & 145 & 121 & 104 & 1 & 76 & 24 \\
\hline QALY-EQ5Dc & 145 & 121 & 104 & 19 & 15 & 66 \\
\hline
\end{tabular}

aBased on 1000 bootstrap replications; bWith a willingness-to-pay threshold of $€ 18,000$; 'Based on the Dutch algorithm for the Euroqol (EQ-5D) scores; dReversed, range 1-10; eIncrease in programme costs from $€ 57.70$ to $€ 141.89$ (MTC group) and from $€ 7.70$ to $€ 82.24$ (MT group) caused by the inclusion of patient costs; ppa: point prevalence abstinence; NB: Net Benefit.

\section{DISCUSSION}

\section{Main findings}

To our current knowledge, this was the first study to determine the costeffectiveness and cost-utility of an Internet-based smoking cessation programme with and without counselling by a practice nurse. The results presented suggest that respondents who received the Internet-based multiple computer tailored pro- 
gramme and tailored counselling by their practice nurse reported significantly more annual healthcare related costs than respondents who received care as usual. A potential explanation for this finding might be that smokers were prompted by the tailored feedback they received to ask for more smoking cessation guidance, e.g. additional counselling sessions with the practice nurse or a prescription for smoking cessation medication. While respondents who received the Internet-based programme only might have had the same tendency, for respondents in the MTC group practice nurses might have additionally been prompted by their patients' visit to offer them more smoking cessation help. As current recommended practice in the Netherlands consists of more than one counselling session (Partnership Stop met Roken, 2009, Pieterse et al., 2001), while as part of MTC practice nurses could offer only one counselling session, this would not be surprising. Interestingly, though, the significant difference in total healthcare-related costs could not be explained by a difference in any particular type of health-care related costs. No significant differences were found, for instance, with regard to costs spent on general practitioner or practice nurse consultations or on the use of smoking cessation aids.

Furthermore, the present study showed that the Internet-based multiple computer tailored smoking cessation programme would probably be the most costeffective of the three treatments under study. Compared with current practice, the incremental costs per abstinent participant were $€ 5,100$. This is slightly more than what was found in previous studies (Salize et al., 2009, Kaper et al., 2006). One of these studies, however, only included costs directly related to the interventions received (Salize et al., 2009), while we conducted our economic evaluation from a broader societal perspective. Nevertheless, the interpretation of the incremental costs per abstinent participant is difficult as no information exists on the amount of money that society is willing to pay per abstinent participant. While a willingness to pay of $€ 18,000$ per QALY is an accepted Dutch cut-off point (Casparie et al., 1998), no such cut-off point exists with regard to abstinence rates. To enable the interpretation of the incremental costs per abstinent participant, future research should aim at identifying an acceptable cut-off point for the willingness to pay per abstinent participant.

When it concerned cost-utilities, the results presented suggest that care as usual would probably be the most preferable of the treatments studied. A potential explanation for this finding might be that the follow-up period of twelve months was not sufficiently long for the beneficial effects of the intervention on smoking abstinence to be translated into detectable changes in quality of life, as recent exsmokers are known to suffer from withdrawal symptoms for about one week post quit attempt (Shiffman et al., 2006). However, previous studies were able to detect a positive association between quitting smoking and quality of life yet during a twelve-month follow-up period (Hays et al., 2010, Sales et al., 2009). Another explanation might be that within Dutch general practices, care as usual for smoking cessation is rather intensive. Anecdotal evidence suggests that practice nurses usually offer four to six consultations as part of smoking cessation care (unpublished). 
While respondents in the MT and MTC groups received either four tailored feedback letters (MT) or three tailored feedback letters, a single counselling session with and a telephone call from a practice nurse (MTC), a mainly Internet-based programme might have been perceived as more distant and less intense than care as usual. As a result, respondents in the UC group might have established a better or stronger social bond with their practice nurse than respondents in the MT or MTC groups. This assumed social bond (Shum et al., 2000) might subsequently have resulted in positive effects on quality of life among UC respondents.

\section{Strengths and limitations}

The present study aimed to contribute to the literature by examining the costeffectiveness and cost-utility of an Internet-based smoking cessation intervention, something which has, to our knowledge, not been done before. In the present study, besides generic quality of life, disease-specific effects of the intervention, i.e. smoking abstinence, were taken into account. To facilitate the comparison of the costeffectiveness of interventions targeting different diseases, effects are usually assessed in terms of quality of life. However, to compare smoking cessation interventions more specifically, disease-specific effect measures might be more informative. That in the present study both effect measures were included can thus be an important strength. Nevertheless, the present study also had its limitations. First, it suffered from relatively high drop-out rates. High rates of attrition seem to be inherent to many Internet-based interventions and drop-out rates of $44 \%$ are not extraordinary when compared to other Internet-based studies (McKay et al., 2008, Shahab and McEwen, 2009, Wangberg et al., 2008, Wangberg et al., 2011). As a consequence, however, there was not sufficient power for us to conduct a complete-case analysis as part of the sensitivity analyses. Secondly, as we expected higher attrition rates in the MTC group, at baseline slightly more respondents were randomized into this intervention group. Attrition rates, however, appeared to be similar among the groups, resulting in a skewed distribution of respondents with 163 respondents in the MTC group, 132 in the MT group and 119 in the UC group. We do no expect that this has biased our results. In fact, our finding of no selective attrition can be considered valuable in the design of future trials.

\section{Conclusions}

The Internet-based multiple computer tailored programme seemed to be the most cost-effective treatment when taking smoking abstinence as the outcome measure. However, the cost-utility, taking quality of life as the outcome measure, of care as usual was most probably highest. To enable the interpretation of the incremental costs per abstinent participant found in the cost-effectiveness analyses, future research should aim at identifying an acceptable cut-off point for the willingness to pay per abstinent participant. 


\section{CHAPTER 8}

The importance of attitude and innovation characteristics in determining the intention to implement a new smoking cessation intervention among Dutch practice nurses: a cross-sectional study

Submitted as: Smit, E.S., Hoving, C., de Vries, H. Determinants of Dutch practice nurses' intention to implement a new smoking cessation intervention: The importance of attitude and innovation characteristics. 


\begin{abstract}
Aims This paper is a report of a study that aimed to identify determinants of Dutch practice nurses' intention to implement a new smoking cessation intervention and to investigate the independent value of attitude and Rogers's innovation characteristics.

Background While effective smoking cessation interventions exist, their implementation in practice is often suboptimal. Previous studies have tried to integrate the I-Change model with the Diffusion of Innovations theory, but none have disentangled the independent value of attitude and Rogers's innovation characteristics in explaining implementation.

Methods In 2010, 56 of 91 practice nurses who participated in a randomized controlled intervention effectiveness trial completed an online questionnaire concerning demographics, patient population characteristics, attitude, innovation characteristics, self-efficacy, perceived social influence and intention to implement the intervention. Recruitment success was defined as the number of patients participating in the trial. To detect differences between intending and non-intending practice nurses independent sample T-tests and Chi-square tests were conducted. Correlation coefficients were calculated to identify associations between potential determinants of the intention to implement. To identify significant determinants logistic hierarchical regression analyses were conducted.

Results While interrelated, both attitude and innovation characteristics were independently, positively associated with practice nurses' intention to implement. Recruitment success and perceived patient support also showed a significant positive association with intention.

Conclusion To increase new interventions' implementation rates, it is important to generate a positive attitude towards the intervention, to convince health professionals of its beneficial characteristics, to aid practice nurses in successfully recruiting smoking patients and to increase perceived patient support.
\end{abstract}




\section{INTRODUCTION}

Smoking is the most preventable cause of illness and premature death in the world (USDHHS, 2004, WHO, 2008). Five million people are dying from tobacco use each year (WHO, 2008), which stresses the importance of smoking cessation. Within the Dutch primary care setting, the care for patients with chronic diseases such as diabetes, asthma or hypertension, for whom smoking cessation is of extra importance, is most often provided by practice nurses (Nederlands Huisartsen Genootschap and Landelijke Huisartsen Vereniging, 2011). Previously, primary care professionals have been shown to be successful in promoting smoking cessation among their patients (Lancaster et al., 2000, Stead et al., 2008) and reasonable evidence exists that nurse-delivered smoking cessation interventions are effective (Rice and Stead, 2008). The public health impact of these interventions, however, is not only dependent on their effect, but also on their reach (Velicer and Prochaska, 1999, de Vries and Brug, 1999, Glasgow et al., 1999), while the implementation of these programmes is often suboptimal (Eccles et al., 2007, Glasgow et al., 2003, Stead et al., 2009). This results in lower cessation rates than ideally possible. To obtain higher smoking cessation rates, it is therefore important to study the cognitive factors associated with the implementation of smoking cessation interventions in practice.

\section{Background}

In previous studies addressing the determinants of the implementation of health behaviour change interventions, socio-cognitive theories such as the I-Change model (De Vries et al., 2003) as well as the Diffusion of Innovations theory (Rogers, 1995) have been frequently applied (Lee, 2004, Pronk et al., 2001, Rogers, 2002, van der Weide and Smits, 2004, Helmink et al., 2011, Hoving et al., 2007b, Puffer and Rashidian, 2004). According to the I-Change model, implementation is determined by the intention to implement, which is determined by three cognitive factors: attitude, social influence and self-efficacy (De Vries et al., 2003). Previous studies indeed showed that health professionals' clinical behaviour could be largely explained by their intention (Eccles et al., 2006), while a positive attitude, positive social norms and a higher self-efficacy have previously been associated with the intention to implement smoking cessation programmes in practice (Hoving et al., 2007b, Puffer and Rashidian, 2004, Helmink et al., 2011). These three motivational factors, in turn, are predicted by several predisposing factors, such as practice nurses' own smoking status and their training in giving smoking cessation advice (Hall et al., 2005, Stead et al., 2009).

The Diffusion of Innovations theory (Rogers, 1995) suggests that the decision to implement a new intervention is a process that occurs over time and consists of multiple phases: knowledge, persuasion, decision, implementation and confirmation (Rogers, 1995). Health professionals first need to be aware of the intervention's existence, then form a favourable or unfavourable attitude towards the intervention, decide to adopt or reject the intervention, start implementing it and ulti- 
mately seek reinforcement of the decision made. Within this process, the attitude towards the intervention is assumed to be based on the perception of five intervention characteristics (i.e. relative advantage, compatibility, complexity, trialability and observability) (Rogers, 1995), which have previously been shown to be related to the implementation of preventive interventions in general (Lee, 2004, Pronk et al., 2001, Rogers, 2002) and smoking cessation interventions in particular (Bolman et al., 2002a, Segaar et al., 2006, Segaar et al., 2007).

Previous implementation studies have tried to integrate Rogers' innovation characteristics within the socio-cognitive concept of attitude (Bolman et al., 2002a, Segaar et al., 2007). In these studies, differences were found between cardiac nurses adhering and non-adhering to a minimal-contact smoking cessation intervention regarding all innovation characteristics (Segaar et al., 2007) and between cardiac nurses intending and non-intending to continue using this intervention regarding the interventions' perceived advantage and complexity (Bolman et al., 2002a). While both studies considered Rogers' innovation characteristics as part of the more general construct of attitude, previous studies of the implementation of innovations in health care have considered attitude to be a characteristic of the intervention's user (i.e. the health professional) and Rogers' concepts as characteristics of the intervention (Fleuren et al., 2004, Helmink et al., 2011). Considering attitude and innovation characteristics as two separate constructs, would lead to the hypothesis that both independently influence implementation. To our knowledge, however, no studies have been conducted that disentangled the independent value of attitude and Rogers's innovation characteristics in explaining the implementation of smoking cessation interventions in practice, while this would provide more specific targets for developing intervention strategies aimed at increasing implementation rates and, consequently, for increasing smoking cessation rates.

\section{Objective of the present study}

The present study aimed to identify determinants of practice nurses' intention to implement a new smoking cessation intervention. In addition we aimed to investigate the relative value of attitude and Rogers's innovation characteristics, next to other cognitive factors as derived from the I-Change model, in explaining the intention to implement this intervention in the general practice setting. We hypothesized that both attitude and innovation characteristics would be independently associated with the intention to implement and that including both constructs separately into the same model explaining the intention to implement would lead to more explained variance than including either of the two constructs alone.

\section{THE STUDY}

\section{Aims}

This paper is a report of a study that aimed to identify determinants of Dutch practice nurses' intention to implement a new smoking cessation intervention and to 
investigate the independent value of attitude and Rogers's innovation characteristics in explaining intention to implement.

\section{Design}

A cross-sectional study was conducted.

\section{Sample/participants}

In June and July 2010, all 91 Dutch general practice nurses who participated in a Randomized Controlled Trial (RCT) testing the effectiveness of the smoking cessation intervention PAS (Personal Advice in Stopping smoking) were invited by e-mail to fill out an online questionnaire. PAS is a smoking cessation intervention consisting of a web-based multiple computer tailored smoking cessation programme and tailored counselling by a practice nurse. From May 2009 to June 2010, these practice nurses offered PAS to their smoking patients, which resulted in a total of 414 smokers taking part in the trial. A more detailed description of the intervention and of the PAS study design is provided elsewhere (Smit et al., 2010).

\section{Data collection}

The online questionnaire consisted of 61 questions and was based on previously developed questionnaires that used the I-Change model (De Vries et al., 2003) and/or Rogers's Diffusion of Innovations theory (Rogers, 1995) as a theoretical framework for studying the intention to implement a smoking cessation programme among general practice staff (Bolman et al., 2002a, Leitlein et al., 2011, Hoving et al., 2007b).

\section{Ethical considerations}

The study was approved by the Medical Ethics Committee of Maastricht University and the University Hospital Maastricht (MEC 08-3-037; NL22692.068.08), and is registered with the Dutch Trial Register (NTR1351).

\section{Data analysis}

All analyses were conducted with SPSS version 17.0.

First, respondents were divided into two groups based on their intention to implement the intervention: intenders (definitely yes, probably) and non-intenders (definitely no, probably not, maybe). Independent sample T-tests and Chi-square tests were conducted to detect differences between intenders and non-intenders with regard to demographics, patient characteristics, recruitment success, attitude, perceived innovation characteristics, social influence and self-efficacy.

Secondly, to identify associations between potential determinants of the intention to implement Pearson's correlation coefficients were calculated for two continuous variables and point-biserial correlations were calculated for the relationships between a dichotomous and a continuous variable. 
Thirdly, hierarchical logistic regression analyses were conducted. We tested three models: 1) a model with concepts from the I-Change model only; 2) a model with concepts from the I-Change model, in which attitude was replaced by Rogers' innovation characteristics; 3) a model with concepts from the I-Change model, including attitude, and Rogers' innovation characteristics. Predisposing factors on both health professional and patient level (i.e. health professionals' smoking status, use of existing smoking cessation guidelines, recruitment success, and the perceived age and educational level of the patient population) were entered into each model first. As this first step was of exploratory nature, we used a backward approach (Field, 2005), whereby variables were excluded from the model based on the likelihood ratio statistic (LR). In the second step, attitude and/or Rogers' innovation characteristics, self-efficacy and social influence were added into the model using the Enter method.

\section{Validity and reliability}

Practice nurses' smoking status (smoking/non-smoking) was assessed, as well as whether they used smoking cessation guidelines before their participation in the trial (yes/no). In addition, respondents were asked to describe their perception of their patient population in terms of age (young/neutral/old) and educational level (low/neutral/high). Recruitment success was defined as the total number of patients in each general practice who participated in the RCT, information known by the research team. Unless stated otherwise, all items described in the following were assessed on a five-point Likert scale (1=totally disagree; $5=$ totally agree).

Attitude towards the intervention was measured with three items, i.e. 'PAS is an effective smoking cessation intervention', 'The Internet is a good medium to aid patients to quit smoking', 'I think that, when combined with a web-based programme, one counselling session with a practice nurse should be sufficient to aid smoking patients to quit' $(\alpha=.59)$. While the Cronbach's alpha of .59 might appear low, this is not extraordinary when measuring psychological constructs with only few items (Field, 2005).

Rogers' innovation characteristics were assessed by four items $(\alpha=.66)$, i.e. 'PAS is an improvement compared with our previous smoking cessation activities' (relative advantage), 'PAS fits within the current way of working in our general practices' (compatibility), 'It was complicated to use PAS in our general practice' (complexity) and 'The use of PAS is beneficial for the general practice's reputation' (observability). Before inclusion in the subscale, the item on complexity was reversed. Trialability was not assessed as all respondents had a trialability opportunity when participating in the RCT. Again, this scale should not be considered unreliable, as a Cronbach's alpha of .66 is not extraordinary when measuring psychological constructs with only few items (Field, 2005).

Self-efficacy was measured with one item, i.e. 'I found it difficult to conduct a counselling session using the PAS counselling protocol'. 
Social influence was measured with two items, assessing whether colleagues stimulated the use of PAS and whether patients were supportive of its use. As no reliable scale could be constructed, both measures were taken into account separately in all analyses.

The intention to implement PAS in the future was measured by one question: 'Whenever PAS becomes nationally available, would you intend to use it in your general practice?' (1=definitely yes; $5=$ definitely not).

\section{RESULTS}

\section{Sample characteristics}

In total, 61 practice nurses filled out our questionnaire (67\% response rate). Of these 61 practice nurses, 56 completed the question about their intention to implement the intervention and were thus included in the data analysis. Twenty-one $(37.5 \%)$ practice nurses could be categorized as intenders and $35(62.5 \%)$ as nonintenders. A small minority of practice nurses reported to currently smoke (6.5\%), while most practice nurses reported to use existing smoking cessation guidelines (91.4\%). On average, practice nurses recruited seven smokers for participation in the RCT (range 0-26).

\section{Differences between intenders and non-intenders}

Table 8.1 provides a detailed description of intending and non-intending practice nurses' characteristics. T-tests and Chi-square test revealed that practice nurses intending to implement reported a significantly higher percentage of younger patients within their patient population. Moreover, they reported significantly less often to have used smoking cessation guidelines than non-intending practice nurses. Among intenders, the average number of patients recruited was significantly higher (10.4) than among non-intenders (4.5). Furthermore, the attitude towards PAS of intenders was significantly more positive and they valued the intervention significantly higher with regard to Rogers' characteristics. Furthermore, perceived patient support and the level of self-efficacy were significantly higher among intenders than among non-intenders.

\section{Correlations between potential determinants of the intention to implement}

The correlation matrix in table 8.2 shows the correlations between all measured determinants of the intention to implement. The patient population's age was negatively related to attitude, while Rogers' innovation characteristics and perceived patient support were both positively related to attitude. Rogers' innovation characteristics were also positively associated with self-efficacy and perceived patient support, while recruitment success showed a positive association with perceived social influence from both colleagues and patients. 


\section{Determinants of the intention to implement}

Three of the predisposing factors remained in the model: practice nurses' use of smoking cessation guidelines, the patient population's age and recruitment success. Of these, only recruitment success was significantly associated with the intention to implement the intervention (OR 1.21; 95\% CI 1.06-1.38). Practice nurses who had recruited more patients were more likely to intend to implement than practice nurses who had recruited fewer patients. These three factors were added into each second model tested.

In the model with predisposing factors and concepts from the I-Change model, attitude was positively associated with the intention to implement, as was the perceived social support from patients. In addition, the positive association between recruitment success and intention to implement remained significant.

In the model with predisposing factors and concepts from the I-Change model, but with attitude replaced by Rogers' innovation characteristics, innovation characteristics showed a significant positive association with the intention to implement. Again, the perceived social support from patients was found to be positively associated with the intention to implement, while the positive association between recruitment success and the intention to implement again remained significant.

In the model with concepts from the I-Change model and Rogers' innovation characteristics, both attitude and innovation characteristics were found to be positively associated with the intention to implement. The positive association between recruitment success and the intention to implement again remained significant, but the association between perceived social support from patients and the intention to implement became non-significant. All three models are presented in table 8.3. 
Table 8.1 Characteristics of intending and non-intending practice nurses $(\mathrm{N}=56)$

\begin{tabular}{|c|c|c|c|c|c|}
\hline & $\begin{array}{l}\text { Intending practice } \\
\text { nurses }(\mathrm{N}=21)\end{array}$ & $\begin{array}{l}\text { Non-intending } \\
\text { practice nurses } \\
\qquad(\mathrm{N}=35)\end{array}$ & $\mathrm{T}$ & $\chi^{2}$ & $\mathrm{p}$-value \\
\hline \multicolumn{6}{|l|}{ Smoking } \\
\hline$\%$ non-smokers & 95.2 & 94.3 & & .02 & .88 \\
\hline \multicolumn{6}{|l|}{ Use of smoking cessation guidelines } \\
\hline$\%$ using no guidelines & 19.0 & 2.9 & & 4.23 & .04 \\
\hline \multicolumn{6}{|l|}{ Patient population } \\
\hline \% young & 23.8 & 5.7 & 2.56 & & .01 \\
\hline$\%$ smoking & 9.5 & 11.4 & .00 & & 1.00 \\
\hline \% Dutch & 66.7 & 80.0 & -1.35 & & .19 \\
\hline$\%$ highly educated & 9.5 & 11.4 & .39 & & .70 \\
\hline Number of patients recruited & $10.4 ; 7.2$ & $4.5 ; 4.6$ & -3.36 & & $<.00$ \\
\hline Attitude (Mean; S.D.) ${ }^{\mathrm{a}}$ & $3.4 ; .5$ & $2.9 ; .5$ & -4.30 & & $<.00$ \\
\hline $\begin{array}{l}\text { Innovation characteristics (Mean; } \\
\text { S.D.) }\end{array}$ & $3.5 ; .3$ & $2.8 ; .5$ & -5.63 & & $<.00$ \\
\hline Self-efficacy (Mean; S.D.)a & $4.0 ; .6$ & $3.4 ; 1.0$ & -2.90 & & .01 \\
\hline \multicolumn{6}{|l|}{ Social influence } \\
\hline Colleagues (Mean; S.D.)a & $2.9 ; .9$ & $2.6 ; .7$ & -.89 & & .38 \\
\hline Patients (Mean; S.D.) ${ }^{\mathrm{a}}$ & $3.2 ; .6$ & $2.4 ; .7$ & -4.54 & & $<.00$ \\
\hline
\end{tabular}

Note: arange from 1-5; significant differences are depicted in bold.

Table 8.2 Correlation matrix

\begin{tabular}{|c|c|c|c|c|c|c|c|c|c|c|}
\hline & 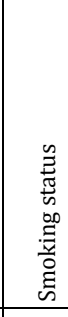 & 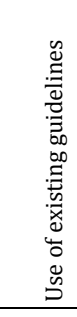 & 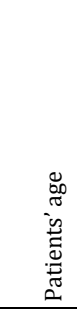 & 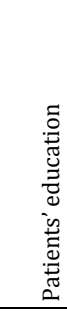 & 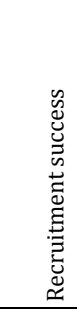 & 曾 & 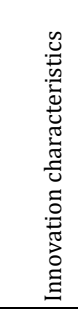 & 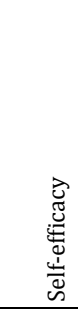 & 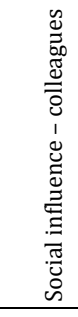 & 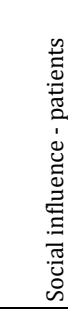 \\
\hline Smoking status & 1.00 & .07 & .02 & -.05 & -.09 & -.04 & -.04 & .09 & -.09 & -.01 \\
\hline Use of existing guidelines & & 1.00 & .23 & .10 & .03 & -.16 & -.21 & -.19 & -.04 & -.12 \\
\hline Patients' age & & & 1.00 & -.08 & -.09 & $-.31^{*}$ & -.25 & -.17 & .22 & -.05 \\
\hline Patients' education & & & & 1.00 & -.08 & -.06 & -.06 & -.09 & -.23 & .07 \\
\hline Recruitment success & & & & & 1.00 & .08 & .25 & .25 & $.43^{* *}$ & $.29^{*}$ \\
\hline Attitude & & & & & & 1.00 & $.44^{* *}$ & .24 & -.20 & $.47^{* *}$ \\
\hline Innovation characteristics & & & & & & & 1.00 & $.43^{* *}$ & .19 & $.49^{* *}$ \\
\hline Self-efficacy & & & & & & & & 1.00 & -.04 & .20 \\
\hline Social support colleagues & & & & & & & & & 1.00 & .17 \\
\hline Social support patients & & & & & & & & & & 1.00 \\
\hline
\end{tabular}

Note: ${ }^{*} \mathrm{p}<, 05 ;{ }^{* *} \mathrm{p}<.01$. 
Table 8.3 Determinants of practice nurses' $(\mathrm{N}=56)$ intention to implement PAS in the future

\begin{tabular}{|c|c|c|c|c|c|c|c|c|c|}
\hline & \multicolumn{3}{|c|}{ I-Change - attitude } & \multicolumn{3}{|c|}{$\begin{array}{l}\text { I-Change - innovation } \\
\text { characteristics }\end{array}$} & \multicolumn{3}{|c|}{$\begin{array}{l}\text { I-Change - attitude and } \\
\text { innovation characteristics }\end{array}$} \\
\hline & \multirow{2}{*}{$\begin{array}{l}\mathrm{R}^{2}=.71 \\
\mathrm{OR}\end{array}$} & \multicolumn{2}{|l|}{$95 \% \mathrm{CI}$} & \multirow{2}{*}{$\begin{array}{l}\mathrm{R}^{2}=.75 \\
\text { OR }\end{array}$} & \multicolumn{2}{|l|}{$95 \% \mathrm{CI}$} & \multirow{2}{*}{$\begin{array}{l}\mathrm{R}^{2}=.81 \\
\text { OR }\end{array}$} & \multicolumn{2}{|l|}{$95 \% \mathrm{CI}$} \\
\hline & & Lower & Upper & & Lower & Upper & & Lower & Upper \\
\hline Use of guidelinesa & 11.23 & .61 & 206.94 & 10.93 & .26 & 457.19 & 30.27 & .15 & 5982.16 \\
\hline $\begin{array}{l}\text { Patient population's } \\
\text { age }\end{array}$ & .36 & .08 & 1.61 & .39 & .09 & 1.76 & .62 & .11 & 3.51 \\
\hline Recruitment success & $1.22^{*}$ & 1.00 & 1.48 & $1.22^{*}$ & 1.00 & 1.50 & $1.34^{*}$ & 1.03 & 1.73 \\
\hline Attitude & $17.76^{*}$ & 1.41 & 224.24 & - & - & - & $33.12^{\wedge}$ & 1.00 & 1102.05 \\
\hline $\begin{array}{l}\text { Innovation } \\
\text { characteristics }\end{array}$ & - & - & - & $35.58^{* *}$ & 2.42 & 522.77 & $61.49^{*}$ & 1.72 & 2194.98 \\
\hline Self-efficacy & 1.12 & .37 & 3.37 & 1.63 & .39 & 6.78 & 3.08 & .62 & 15.37 \\
\hline \multicolumn{10}{|l|}{ Social support } \\
\hline Colleagues & 1.13 & .33 & 3.87 & .80 & .19 & 3.35 & 1.13 & .20 & 6.40 \\
\hline Patients & $9.00^{*}$ & 1.13 & 71.51 & $8.65^{*}$ & 1.18 & 63.45 & 18.21 & .50 & 658.84 \\
\hline
\end{tabular}

Note: aReference category no use of guidelines; ${ }^{\wedge} \mathrm{p}=.05 ;{ }^{*} \mathrm{p}<, 05 ;{ }^{* *} \mathrm{p}<.01$.

\section{DISCUSSION}

\section{Limitations}

In the study, some limitations were present. First, the study was of cross-sectional nature. Therefore, we were unable to draw conclusions about the causality of the associations found. A related limitation is that the study was relatively underpowered to conduct regression analyses due to the relatively small sample size, as demonstrated by several wide confidence intervals. While the results presented should thus be interpreted with caution, they can be valuable in guiding future longitudinal studies with larger samples of practice nurses.

Secondly, the study was conducted amongst Dutch practice nurses who participated in a trial testing the effectiveness of a new smoking cessation intervention developed in the Netherlands. As all participating practice nurses had yet agreed to adopt the intervention for the effectiveness trial period, these practice nurses might have viewed the intervention more positive than the average Dutch practice nurse would have. In line with a previous study, however, we would expect the differences between intenders and non-intenders as presented in this manuscript to be even more profound when all Dutch practice nurses would have participated (Hoving et al., 2007b).

Thirdly, we were unable to study the determinants of actual implementation of the intervention as it was not yet nationally available. A recent systematic review, however, showed intention to be an important predictor of health professionals' clinical behaviour, explaining a similar proportion of the variance in behaviour found in literature among non-health professionals (Eccles et al., 2006). The inten- 
tion to implement can therefore be considered a reliable precursor of actual implementation.

Lastly, self-efficacy was measured with one item only, which is not a reliable measure of the construct. Within the present study, we aimed to minimize practice nurses' burden of filling out the questionnaire, but future studies should aim to assess self-efficacy with at least three items.

\section{Discussion of main findings}

The present study is the first that showed that both attitude and Rogers' innovation characteristics are independently important in explaining practice nurses' intention to implement a new smoking cessation intervention in the general practice setting. In addition, recruitment success and perceived support from patients are both suggested to be significant determinants of the intention to implement.

While causality could not be established within the present study, it might thus be important to generate a positive attitude among health professionals towards implementation and to convince them of a new intervention's beneficial characteristics in order to increase implementation rates. Within the present study, practice nurses were informed about the new intervention by the research team. The I-Change model, however, suggests that the source of the message is an important predictor of attitude (De Vries et al., 2003), while Rogers has previously suggested that the use of champions might facilitate the dissemination of preventive interventions (Rogers, 2002). To increase a positive attitude towards implementation, it might therefore be better to disseminate information about a new intervention for practice nurses via the general practitioners they are working for, instead of via the researchers they barely know. Future studies should aim to identify whether this is indeed the case. Furthermore, a previous study showed that practice nurses' satisfaction with their current smoking cessation activities was negatively associated with their intention to adopt a new smoking cessation intervention (Leitlein et al., 2011). Conceivably, practice nurses based their attitude towards implementation of the intervention on their satisfaction with the intervention during the initial trial period (i.e. the RCT). It would be interesting to assess the role satisfaction plays in determining implementation as well.

In line with a previous study (Hoving et al., 2007b), practice nurses who were more successful in recruiting patients during the RCT reported a higher intention to implement the intervention than those who were less successful. Within our RCT, all participating practice nurses were provided with many recruitment materials, such as desk displays, recruitment letters, business cards, recruitment texts for their websites and posters to help them to recruit smoking patients (Smit et al., 2010). Moreover, incentives were sent to practice nurses after recruiting five and ten patients. Still, however, not all practice nurses were able to recruit smoking patients to participate in the RCT testing the intervention's effectiveness. The positive correlation found between perceived social support from colleagues and recruitment success suggests that practice nurses who experienced more support 
from their colleagues were more successful in recruiting smoking patients. However, both intending and non-intending practice nurses scored relatively low on the perceived support from colleagues, i.e. lower than three on a five-point scale. Here thus seems to be room for improvement. As a previous study showed that a more active role of general practice staff resulted in a higher intention to implement a smoking cessation expert system (Hoving et al., 2007b), a more active involvement of the GP or practice assistant with in the recruitment process might be a strategy to increase recruitment rates and to ultimately increase the intention to implement the intervention in the general practice. Another possibility for relatively low rates of recruitment might be that smoking patients were not willing to quit smoking. This possibility warrants further exploration in future studies.

More perceived patient support also turned out to be associated with a positive intention to implement. Creating more perceived patient support might be a strategy to boost practice nurses' intention to implement. As in the present study both intending and non-intending practice nurses did not experience much support from their patients, there appears to be room for improvement. One possibility to increase perceived patient support might be to disseminate testimonials of patients describing their positive experiences with the intervention among health professionals via regular e-newsletters or a study website. In addition, more intensive involvement of patient organizations in the development of the intervention and explicitly showing their involvement to practice nurses (e.g. in promotion materials), may be another strategy that could further stimulate implementation (Rogers, 2002). This may give practice nurses the idea that (their) patients approve of the intervention, leading to more perceived patient support.

\section{Conclusion}

The present study is the first that showed that both attitude and Rogers' innovation characteristics are independently important in explaining practice nurses' intention to implement a new smoking cessation intervention. In addition, recruitment success and perceived support from patients are both suggested to be significant determinants of the intention to implement. To increase implementation rates of new smoking cessation interventions, it might thus not only be important to generate a positive attitude towards implementation and to convince health professionals of the beneficial characteristics of a new intervention, e.g. by involving general practitioners in the dissemination process, but also to aid practice nurses in successfully recruiting patients, e.g. by involving the other members of the general practice's team in the recruitment process, and to increase practice nurses' perceived patient support, e.g. by disseminating their positive experiences with the intervention via testimonials. 


\section{CHAPTER 9}

The role of desire, duty and intention in predicting attempts to quit smoking

Published as: Smit, E.S., Fidler, J.A., West, R. (2010). The role of desire, duty and intention in predicting attempts to quit smoking, Addiction, 106(4), 844-851. 


\section{ABSTRACT}

Aims Motivation to quit smoking predicts quit attempts, although little is known about the role played by its different aspects. This study assessed the predictive value of desire, duty and intention to quit, three different aspects of motivation.

Design A longitudinal study was conducted involving a nationally representative sample of smokers assessed at baseline and 3 and 6 months later. Baseline assessment took place by face-to-face computer-assisted interviews; follow-up assessments by postal questionnaires.

Setting England.

Participants From April 2008 to June 2009, a total of 5593 adult smokers were recruited; 1263 were followed-up at 3 months and 1096 at 6 months.

Measurements Three dichotomous measures of motivation to quit (wanting to quit, believing one ought to quit, intention to quit soon) were taken at baseline. Whether a subsequent quit attempt was made was recorded at 3- and 6-month follow-up.

Findings More smokers believed they ought to quit smoking than wanted to or intended to soon (39.0, 29.3 and 23.5\%, respectively). Desire and intention were independent predictors of quit attempts at both follow-ups, whereas combining them did not add predictive value and duty was not a predictor. While the predictive value of desire or intention alone disappeared when accompanied by duty, their combination was robust against its negative effect.

Conclusions Desire and intention independently positively predict quit attempts, while duty appears to mitigate their effect. It would be worth monitoring all three aspects of motivation when evaluating the impact of smoking cessation interventions on motivation to quit. 


\section{INTRODUCTION}

Smoking cessation campaigns often focus on creating a sense of 'duty', a belief that one ought to quit for the sake of one's health, the health of one's family or because smoking is in some sense 'bad'. However, there is little empirical evidence to suggest that instilling such beliefs results in behaviour change. A new theory of motivation, PRIME theory (Plans, Responses, Impulses, Motives, Evaluations), provides a theoretical basis for understanding how different aspects of the motivational system might be expected to influence behaviour. According to PRIME theory, multiple levels of motivation can be discerned: basic 'impulses and inhibitions' as direct responses to immediate stimuli, 'motives/desires' (feelings of want and need relating to an imagined future), 'evaluations' (beliefs about what is good or bad) and 'plans' (intentions regarding future actions) (West, 2009, West and Hardy, 2006). PRIME theory argues that attempts to quit occur when at a given moment feelings of desire to quit exceed a competing desire to continue smoking, while evaluations such as beliefs about duty do not play a role in generating quit attempts, unless they result in the smoker desiring to quit (West, 2009, West and Hardy, 2006). Indeed, smokers themselves appear to believe that an unambivalent desire to quit is necessary for a quit attempt to have the potential to succeed (Balmford and Borland, 2008). Wanting to quit smoking (motive-level) has been well established as a predictor of quit attempts, as has intention to quit in the near future (plan-level) (Armitage and Conner, 2001, Hyland et al., 2006, Norman et al., 1999). However, PRIME theory predicts that intention would only influence quitting behaviour if accompanied by a want or need to quit, as beliefs about duty (evaluation-level) and intention are hypothesized to only influence quitting behaviour through desire (West, 2009, West and Hardy, 2006). There appears to be no research to date differentiating these three aspects of motivation, although disentangling the roles they play could be useful in identifying optimal markers of motivation to quit and in ultimately designing effective interventions to promote quitting behaviour.

In 2007, 21\% of all adults aged 16 years and over in England reported that they were smoking (The NHS Information Centre, 2009). Of these smokers, the majority (67\%) recognized the (health) benefits of quitting smoking and expressed a wish to stop (The NHS Information Centre, 2009, West et al., 2001), as has been found in other western countries (Siahpush et al., 2006). However, it is unknown whether these national figures represent smokers who specifically want to quit smoking, smokers who believe they ought to, or both. As several countries, such as Great Britain, Ireland and the Netherlands, have adopted a national smoking ban in public places, public norms are shifting towards non-smoking (Orbell et al., 2009) and smokers are likely to believe that they ought to quit, even though they might not want to. This may lead to an overestimation of the percentage of smokers genuinely motivated to quit smoking when measures are used that do not make a distinction between these different motivational aspects. This overestimation of the 
percentage of smokers wanting to quit might lead to a misunderstanding of the effectiveness of smoking cessation interventions.

The present study concerned the extent to which different aspects of motivation to quit smoking play a role in predicting quit attempts. It aimed to determine the prevalence of intention, desire and beliefs about duty to quit smoking when smokers had the opportunity to answer all three questions simultaneously and to examine the predictive value of each of these aspects of motivation for quit attempts in the succeeding 3 and 6 months. It was hypothesized that fewer smokers would report a desire to quit smoking than found in previous studies, as within the present study smokers were given the opportunity to distinguish between their intention to quit, their desire and feelings of duty to quit. In addition, it was hypothesized that a desire to quit smoking would be predictive of future quit attempts, while duty and intention would only have an influence through desire.

Making an attempt to quit is an essential step in the process of smoking cessation. The success of a quit attempt, however, is a different matter. A quit attempt's success is predicted mainly by the extent to which a smoker is addicted to nicotine and motivation appears not play an important role in whether or not permanent abstinence is achieved (McEwen et al., 2008, West, 2009). Therefore, the present study focused only on the influence of motivation on quit attempts, not on its influence on the success of these attempts.

\section{METHODS}

\section{Sample and procedure}

The study was part of the Smoking Toolkit Study (http://www.smokinginengland.info), a series of monthly surveys intended to provide up-to-date information about smoking and smoking cessation patterns in England (West, 2006). The surveys were conducted by the British Market Research Bureau using a random location sampling design, with initial random selection of grouped output areas, stratified by ACORN characteristics (http://www.caci.co.uk/acorn/acornmap.asp, archived at http://www.webcitation.org/5mcwEK4wN) and region. Subsequently, trained interviewers held face-to-face computer assisted interviews with one member of each household, based on quotas taking into account the likelihood of the household member being at home (e.g. age, gender, part-time working). All smokers who agreed to be re-contacted were sent postal follow-up questionnaires 3 and 6 months after the baseline assessment.

Data used were collected from April 2008 until June 2009. In total, 5593 smokers were interviewed at baseline, of whom 1263 (22.6\%) and 1096 (19.6\%) completed the 3-month and 6-month follow-up questionnaires, respectively. Eight hundred and twenty-four $(824 ; 14.7 \%)$ respondents completed both follow-up questionnaires. 


\section{Baseline measurement}

Three socio-demographic variables were measured at baseline: gender, age and social group.

Smoking behaviour was measured by one item asking: 'Which of the following best applies to you?' [I smoke cigarettes (including hand-rolled) every day; I smoke cigarettes (including hand-rolled), but not every day; I do not smoke cigarettes at all, but I do smoke tobacco of some kind (e.g. pipe or cigar); I have stopped smoking completely in the last year; I stopped smoking completely more than a year ago; I have never been a smoker (i.e. smoked for a year or more), don't know]. Only those respondents indicating that they smoked cigarettes (including hand-rolled) every day or not every day were included in the present study. Respondents were asked to report how many cigarettes per day (or per week or month for non-daily smokers) they usually smoked.

Severity of nicotine dependence was measured by the Fagerström Test for Nicotine Dependence (FTND) (Heatherton et al., 1991), resulting in scores ranging from 0 (not addicted) to 10 (highly addicted).

The three measures of motivation were assessed using dichotomous items with which the respondent could either agree or disagree, i.e. desire was measured with the item 'I want to stop smoking'; duty with 'I ought to stop smoking'; and intention with 'I intend to stop smoking soon'. Subsequently, these three measures were combined into one composite variable, 'Type of motivation', whereby all possible combinations were converted into a single variable. Dichotomous measures were used rather than scale measures to simplify completion for the respondents but also, importantly, because it would not have been possible to devise response scales that were equivalent for the three concepts. That is, one can ask about the strength of 'want' but not about the strength of 'ought'. The closest one could come to the latter would be asking about the strength of the 'belief that one ought', but arguably that is not the same thing. This might have biased the results in favour of our hypothesis. To avoid this, dichotomous measures were used.

\section{Follow-up measurements}

At 3 and at 6 months' follow-up respondents were asked to report quit attempts in the past year using the question: 'How many serious attempts to quit smoking have you made in the last 12 months? By serious attempt I mean you decided that you would try to make sure you never smoked again. Please include any attempt that you are currently making.' Those reported having made at least one quit attempt were then asked how long ago the quit attempt had started (in the last week; more than a week up to a month; more than 1 month and up to 2 months; more than 2 months and up to 3 months; more than 3 months and up to 6 months; more than 6 months and up to a year; can't remember). These two questions were used to assess quit attempts made in the past 3 months for the 3-month follow-up and in the last 6 months for the 6-month follow up. 


\section{Statistical analysis}

First, descriptive analyses were carried out to determine the sample's characteristics and to calculate the percentages of respondents reporting each type of motivation to quit. Thereafter, a comparison was made between those followed-up and those lost to follow-up after 3 and 6 months. In addition, tetrachoric correlations were calculated between the three different types of motivation and the frequency of each possible combination of the different types of motivation to quit was determined.

Secondly, multiple logistic regression analyses were conducted to investigate whether self-reported measures of motivation and our main outcome measures, i.e. whether a quit attempt had been undertaken since baseline, measured at both follow-up points, differed by sociodemographic and/or smoking variables. In these analyses the three different motivational measures and the main outcome measures functioned as dependent variables and socio-demographic and smoking variables measured at baseline as independent variables. Those variables that came out as predictors of both motivation and quit attempts were included as covariates in subsequent analyses.

Thirdly, simple logistic regression analyses were carried out to assess bivariate associations between the three different types of motivation and quit attempts at both follow-up moments. Multiple logistic regression analyses with all three types of motivation entered simultaneously were conducted to determine the unique predictive power of each type of motivation. Lastly, to investigate the predictive validity of each potential combination of motivational aspects, the influence of Type of motivation' on quit attempts at 3- and 6-month follow-ups was assessed using multiple logistic regression analyses.

Data were analysed using SPSS version 15.0.

\section{RESULTS}

\section{Sample characteristics}

Table 9.1 shows the characteristics of the baseline sample. In table 9.2 a comparison is made between the samples followed-up and those lost to follow-up with regard to their baseline characteristics. As is shown, compared with those who completed the 3-month and 6-month follow-up questionnaires, those lost to follow up were slightly younger, more likely to be male and smoked slightly fewer cigarettes per day.

In addition, table 9.1 shows that the most commonly reported type of motivation to quit was duty (39.0\%), with desire and intention being less frequent $(29.3$ and $23.5 \%$, respectively). Desire, duty and intention were associated moderately with each other, as is shown in Table 9.3. The odds of reporting an intention to quit were 5.93 [95\% confidence interval (CI) 4.48-7.85] greater in those who expressed a desire to quit compared with those who did not and 1.86 (95\% CI 1.43-2.42) greater in those who reported a sense of duty to quit compared with those who did 
not. The odds of reporting a desire to quit were 2.41 (95\% CI 1.88-3.09) greater in those who felt it their duty to quit compared with those who did not.

Table 9.1 Sample characteristics at baseline

\begin{tabular}{lc}
\hline & $\mathrm{N}=5593$ \\
\hline Age, mean (SD) & $41.7(16.2)$ \\
Male, percent (N) & $47.6(2660)$ \\
Social grade, percent (N)a & \\
$\quad$ AB & $10.2(572)$ \\
C1 & $21.7(1216)$ \\
C2 & $24.5(1371)$ \\
D & $19.1(1066)$ \\
E & $24.5(1368)$ \\
Number of cigarettes smoked per day, mean (SD) & $13.5(8.6)$ \\
Tried to quit in the past year, percent (N) & $36.0(2008)$ \\
FTND score, mean (SD) & $3.3(2.3)$ \\
Respondents reporting each type of motivation, percent (N) & \\
No motivation & $40.3(2254)$ \\
Intention & $23.5(1315)$ \\
Desire & $29.3(1641)$ \\
Duty & $39.0(2179)$ \\
Respondents with each combination of motivational types, percent & \\
(N) & \\
Duty only & \\
Desire only & \\
Intention only & $19.9(1114)$ \\
Desire \& duty & $9.4(523)$ \\
Intention \& duty & $7.1(398)$ \\
$\quad$ Desire \& intention & $6.9(387)$ \\
\hline
\end{tabular}

Note: $n . a .=$ not applicable ; ${ }^{\mathrm{ABB}}=$ higher and intermediate professional/managerial; $\mathrm{C} 1=$ supervisory, clerical, junior managerial/administrative/professional; $\mathrm{C} 2=$ skilled manual workers; $\mathrm{D}=$ semi-skilled and unskilled manual workers; $E=$ on state benefit, unemployed, very low paid workers. 
Table 9.2 A comparison of those followed up and those lost to follow-up after three and six months

\begin{tabular}{|c|c|c|c|c|}
\hline & $\begin{array}{c}\text { Followed up after } \\
3 \text { months } \\
(\mathrm{N}=1263)\end{array}$ & $\begin{array}{l}\text { Lost to follow-up } \\
\text { after } 3 \text { months } \\
\quad(\mathrm{N}=4330)\end{array}$ & $\begin{array}{c}\text { Followed up after } \\
6 \text { months } \\
(\mathrm{N}=1096)\end{array}$ & $\begin{array}{l}\text { Lost to follow-up } \\
\text { after } 6 \text { months } \\
\quad(\mathrm{N}=4497)\end{array}$ \\
\hline Age, mean (SD) & $45.5(15.5)$ & $40.6(16.2)$ & $47.6(14.9)$ & $40.3(16.2)$ \\
\hline Male, percent $(\mathrm{N})$ & $42.4(536)$ & $49.1(2124)$ & $43.7(479)$ & $48.5(2181)$ \\
\hline Social grade, mean (SD)a & $2.7(1.4)$ & $2.8(1.3)$ & $2.8(1.3)$ & $2.7(1.3)$ \\
\hline $\begin{array}{l}\text { Number of cigarettes smoked per } \\
\text { day, mean (SD) }\end{array}$ & $14.0(8.6)$ & $13.3(8.6)$ & $14.5(9.0)$ & $13.3(8.5)$ \\
\hline $\begin{array}{l}\text { Tried to quit in the past year, } \\
\text { percent }(\mathrm{N})\end{array}$ & $35.7(451)$ & $36.0(1557)$ & $35.3(386)$ & $36.2(1622)$ \\
\hline FTND score, mean (SD) & $3.4(2.3)$ & $3.3(2.3)$ & $3.4(2.3)$ & $3.3(2.3)$ \\
\hline
\end{tabular}

Note: significant differences between the samples $(\mathrm{p}<.05)$ are depicted in bold; ${ }^{\text {FFor }}$ these analyses, social grade was considered a continuous measure ranging from 1 (lowest social grade) to 5 (highest social grade).

Table 9.3 Tetrachoric correlations between the three different measures of motivation

\begin{tabular}{lccc}
\hline & Desire to quit & Intention to quit & Duty to quit \\
\hline Desire to quit & $.341^{* *}$ & $.341^{* *}$ & $.204^{* *}$ \\
Intention to quit & $.204^{* *}$ & & $.143^{* *}$ \\
Duty to quit & $.143^{* *}$ & \\
\hline
\end{tabular}

Note: Tetrachoric correlations are equivalent to Pearson correlations except that they involve dichotomous data; ${ }^{*} \mathrm{p}<.05 ;{ }^{* *} \mathrm{p}<.01 ;{ }^{* * *} \mathrm{p}<.001$

\section{Differences in motivation and behaviour by socio-demographic and smoking variables}

Results from the logistic regression analyses carried out to investigate whether selfreported dichotomous measures of motivation differed by socio-demographic and/or smoking variables indicate that the older respondents were, the less likely they were to report an intention to quit smoking soon [odds ratio (OR) $=0.98,95 \%$ CI 0.98-0.99] and a desire to quit smoking (OR=0.99, 95\% CI0.99-0.99), but the more likely they were to report a sense of duty to quit (OR=1.00, 95\% CI 1.00-1.01). Female respondents were more likely than male respondents to report a desire to quit (OR=1.14, 95\% CI 1.01-1.28) and feelings of duty to quit (OR=1.22, 95\% CI 1.11-1.33). Respondents with lower social grades were less likely to report a desire to quit, an intention to quit and a sense of duty to quit (OR=0.94, 95\% CI 0.90-0.98; $\mathrm{OR}=0.90,95 \% \mathrm{CI} 0.86-0.94$; and $\mathrm{OR}=0.84,95 \% \mathrm{CI} 0.81-0.87$, respectively) than respondents with higher social grades. The more cigarettes respondents reported they smoked per day, the more likely they were to report a sense of duty to quit $(\mathrm{OR}=1.01,95 \% \mathrm{CI} 1.00-1.02)$, but the less likely they were to report a desire to quit $(\mathrm{OR}=0.99,95 \% \mathrm{CI} 0.98-1.00)$ and an intention to quit smoking soon $(\mathrm{OR}=0.99,95 \%$ CI $0.98-1.00$ ). In addition, the more addicted to nicotine, the less likely respondents were to report an intention to quit smoking soon (OR=0.96, 95\% CI 0.93-0.99). 
None of the socio-demographic or smoking variables predicted whether respondents reported having made a quit attempt at 3-month follow-up. One variable predicted whether they made a quit attempt during the 6-month follow-up period: the number of cigarettes smoked per day, i.e. the more cigarettes one smoked daily, the less likely he or she would be to make a quit attempt (OR=0.99, 95\% CI $0.97-$ 1.00). This variable was thus included as a covariate in subsequent regression analyses concerning quit attempts at 6-month follow-up.

\section{Predictive power of the three types of motivation regarding attempts to quit smoking}

In separate logistic regression analyses with each measure of motivation entered on its own, desire and intention to quit both predicted quit attempts at 3 and 6 months' follow-up, whereas duty did not (Table 9.4). In a logistic regression in which desire, intention and duty were entered together, desire and intention predicted independently attempts to quit at 3 and 6 months, while duty was associated negatively with quit attempts (Table 9.5).

Figure 9.1 shows the percentages of smokers in each category of the 'Type of motivation' composite measure who reported to have made a quit attempt during the 3- and 6-month follow-up period, respectively. Those who reported that they felt it their duty to quit were no more likely to attempt to quit than those who did not report any motivation to quit at baseline. Those who reported an intention to quit soon or a desire to quit were approximately twice as likely to try to quit compared with those reporting a sense of duty to quit, as were those who reported both an intention and a desire to quit. Reporting a sense of duty to quit smoking reduced the likelihood of quitting in those who also reported either a desire or an intention to quit, but not in those who reported both desire and intention to quit (Table 9.6).

Table 9.4 Bivariate associations of the three motivational measures with quit attempts made at three- and six-month follow-up

\begin{tabular}{|c|c|c|c|c|}
\hline & \multicolumn{2}{|c|}{ Quit attempts - 3 months } & \multicolumn{2}{|c|}{ Quit attempts - 6 months } \\
\hline & OR & $95 \% \mathrm{CI}$ & OR & $95 \% \mathrm{CI}$ \\
\hline Intention to quit smoking soon & $2.50^{* * *}$ & $1.87-3.35$ & $2.04^{* * *}$ & $1.44-2.90$ \\
\hline Number of cigarettes smoked per day & n.a. & n.a. & .99 & $.97-1.01$ \\
\hline Gendera & n.a. & n.a. & n.a. & n.a. \\
\hline Desire to quit smoking & $2.14^{* * *}$ & $1.61-2.83$ & $2.08 * * *$ & $1.49-2.89$ \\
\hline Number of cigarettes smoked per day & n.a. & n.a. & .99 & $.97-1.01$ \\
\hline Gendera & n.a. & n.a. & n.a. & n.a. \\
\hline Duty to quit smoking & .81 & $.61-1.07$ & .90 & $.66-1.22$ \\
\hline Number of cigarettes smoked per day & n.a. & n.a. & .99 & $.97-1.01$ \\
\hline Gendera & n.a. & n.a. & n.a. & n.a. \\
\hline
\end{tabular}

Note: n.a.=not applicable; ${ }^{*} \mathrm{p}<.05 ;{ }^{* *} \mathrm{p}<.01 ;{ }^{* * *} \mathrm{p}<.001 ;{ }^{a}$ Male respondents formed the reference category. 
Table 9.5 Independent predictive value of the three motivational measures regarding quit attempts made at three- and six-month follow-up

\begin{tabular}{lcccc}
\hline & \multicolumn{2}{c}{ Quit attempts -3 months } & \multicolumn{2}{c}{ Quit attempts - 6 months } \\
& OR & $95 \% \mathrm{CI}$ & OR & $95 \% \mathrm{CI}$ \\
\hline Intention to quit smoking soon & $2.15^{* * *}$ & $1.57-2.96$ & $1.97^{* * *}$ & $1.43-2.71$ \\
Desire to quit smoking & $1.81^{* * *}$ & $1.33-2.48$ & $1.69^{* *}$ & $1.24-2.31$ \\
Duty to quit smoking & $.64^{* *}$ & $.47-.86$ & $.71^{*}$ & $.54-.96$ \\
Number of cigarettes smoked per day & n.a. & n.a. & .99 & $.98-1.01$ \\
Gendera & n.a. & n.a. & n.a. & n.a. \\
\hline
\end{tabular}

Note: n.a.=not applicable; ${ }^{*} \mathrm{p}<.05 ;{ }^{* *} \mathrm{p}<.01 ;{ }^{* * *} \mathrm{p}<.001 ;{ }^{a}$ Male respondents formed the reference category.

Table 9.6 The predictive value of type of motivation regarding quit attempts made at three- and sixmonth follow-up

\begin{tabular}{lcccc}
\hline & \multicolumn{2}{c}{ Quit attempts - 3 months } & \multicolumn{2}{c}{ Quit attempts - 6 months } \\
& OR & $95 \%$ CI & OR & $95 \%$ CI \\
\hline No motivation (reference) & 1.00 & - & 1.00 & - \\
Duty only & .70 & $.45-1.07$ & .79 & $.53-1.17$ \\
Desire only & $2.49^{* * *}$ & $1.54-4.05$ & $2.26^{* *}$ & $1.37-3.74$ \\
Intention only & $2.94^{* * *}$ & $1.77-4.90$ & $3.27^{* * *}$ & $1.93-5.53$ \\
Desire \& duty & 1.00 & $.55-1.82$ & 1.51 & $.90-2.55$ \\
Intention \& duty & 1.09 & $.49-2.43$ & 1.38 & $.70-2.74$ \\
Desire \& intention & $2.64^{* *}$ & $1.48-4.71$ & $2.26^{*}$ & $1.15-4.42$ \\
Desire, intention \& duty & $3.01^{* * *}$ & $1.93-4.70$ & $2.46^{* * *}$ & $1.55-3.88$ \\
Number of cigarettes smoked per day & n.a. & n.a. & 1.00 & $.98-1.01$ \\
Gendera & n.a. & n.a. & n.a. & n.a. \\
\hline
\end{tabular}

Note: ${ }^{*} \mathrm{p}<.05 ;{ }^{* *} \mathrm{p}<.01 ;{ }^{* * *} \mathrm{p}<.001 ;$ aMale respondents formed the reference category.

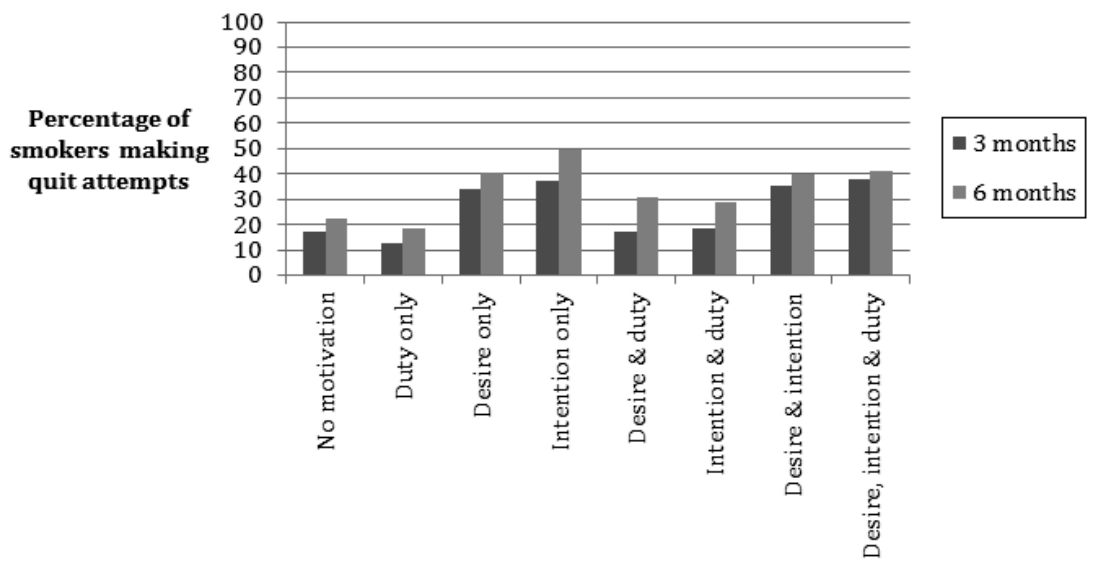

Type of motivation

Figure 9.1 The percentages of smokers who reported having made a quit attempt during the three- and six-month follow-up periods according to their type of motivation to quit at baseline 


\section{DISCUSSION}

Duty was the most commonly reported motive for quitting smoking. However, both desire and intention were significant independent predictors of quit attempts, while duty was not. If duty accompanied reported desire or intention to quit, it even mitigated the predictive power of either the other motivational aspect. Combining intention and desire did not add predictive power compared with either of the two alone, but did prevent duty from undermining it.

The finding that duty did not predict quit attempts is in line with predictions from PRIME theory. However, the fact that duty mitigated the effect of desire and intention was not predicted. Based on these findings, the theory may need to be modified to include a negative association between duty and behaviour. This would be consistent with previous research, suggesting that a sense of autonomy is important for health behaviour change and a sense of external pressure is potentially counterproductive (Williams et al., 2002, Williams et al., 2006a). This is what would be predicted by self-determination theory (SDT), which proposes that extrinsic motivation (in this case, duty might be considered an extrinsic type of motivation) often undermines the positive effect of intrinsic motivation (in this case desire and potentially also intention) (Deci and Ryan, 2008). Another possibility is that the findings reflect the questionnaire format used. If a smoker is asked at the same time whether she or he wants or intends to quit smoking and whether she or he feels she or he ought to quit, some smokers might interpret 'ought' as 'I ought, but do not want/intend'. This could potentially be tested by separating the items physically from each other in the questionnaire.

Moreover, that intention appeared to predict quit attempts independently from desire was not expected based on PRIME theory either. Perhaps the speculation in the theory that intention only has an influence on behaviour through desire needs to be reconsidered. Instead, it may be that intention has a direct positive influence on behaviour. It may be that in some sense 'commitment' to something that one has decided to do carries an autonomous motivational value that goes beyond the desire that led to that commitment in the first place. However, it is also possible that 'commitment' does, as PRIME theory suggests, have its influence on behaviour through a momentary desire to quit smoking, but that this process is not revealed until the very moment at which it unfolds. The finding that intention did not add predictive value when combined with desire suggests that this might be the case. However, this again is something that requires further research. It would be interesting to examine, for example, to what extent intention and desire predict 'planned' and 'unplanned' quit attempts (West and Sohal, 2006).

From a practical point of view, the findings suggest that interventions aimed at promoting quit attempts need to go beyond creating the belief that smokers ought to quit smoking and make them want to quit and/or intend to quit smoking in the near future. Further research is needed into what specific types of strategies can generate a desire or intention to quit while minimizing a residual sense of duty. In 
addition, the results suggest that it is helpful to measure all three aspects of motivation (desire, duty and intention) simultaneously when evaluating smoking cessation interventions.

The study had a number of limitations. First of all, it suffered from relatively high dropout rates when comparing these rates with other population studies (Hyland et al., 2006, Zhou et al., 2009). Dropout occurred both at baseline, where $10 \%$ of baseline respondents were unwilling to be re-contacted, and at follow-up through non-response to questionnaires, either as a result of change of address or failure to return the questionnaire. However, despite the low follow-up rate, only small differences were found between the baseline sample and the samples followed-up at 3 and 6 months, respectively, and it is difficult to conceive of a plausible mechanism that could link the low response rates to the specific pattern of results obtained. Secondly, some degree of recall bias may have occurred when respondents had to report quit attempts at follow-up. Failed quit attempts tend to be forgotten, and the sooner they fail the quicker they are forgotten (Berg et al., 2010, Gilpin and Pierce, 1994). However, the process by which this type of bias could have led to the results obtained is unclear. Also, that the same pattern of results was found with self-reported quit attempts over two different time-periods (3 and 6 months) suggests that forgetting did not play a role. Thirdly, dichotomous measures of motivation were used. The reason for doing this has been explained in the Methods section. Again, although this may have reduced the predictive power of all the variables (Field, 2005, Preston and Colman, 2000), it is not clear how this would have reduced the predictive power of duty more than of desire or intention. Moreover, it is worth mentioning that our dichotomous measure of desire performed similarly in predicting quit attempts to scale measures used previously in the literature (Borland et al., 2010, West et al., 2001, Zhou et al., 2009), so it is not clear whether in this case dichotomization impaired prediction. Nevertheless, it would be useful to determine whether the results presented here could be replicated with continuous measures of motivation. 


\section{CHAPTER 10}

\section{Does a typical contemplator exist?}

\section{Three clusters of smokers in contemplation}

Published as: Smit, E.S., Hoving, C., de Vries, H. (2010). Does a Typical Contemplator Exist? Three clusters of smokers in contemplation, Health Education Research, 25(1), 61-73. 


\section{ABSTRACT}

Background The aim of this study is to test whether subtypes exist among smokers in contemplation.

Methods Data from 194 adult smokers that participated in a randomized controlled trial testing the effectiveness of a computer-tailored smoking cessation programme in Dutch general practices were used for secondary analysis. Cluster analysis was conducted based on baseline scores on pros and cons of quitting and self-efficacy to quit. Clusters were crosssectionally compared for demographic variables and smoking characteristics with analyses of variance (ANOVA) and Chi-square tests. Logistic and multinomial regression analyses were used for longitudinal comparison for smoking behaviour and stage of change at 6 months follow-up.

Results Three clusters were identified: Early, Progressing and Disengaged Contemplators. Clusters differed significantly on all clustering variables $(P<0.001)$. Disengaged smokers were significantly less addicted than Early Contemplators. Cluster membership was not predictive of outcome measures.

Discussion No subtype was identified representing the Classic Contemplator, scoring high on both pros and cons of quitting and low on self-efficacy, as found in previous studies among US samples. The predictive validity of the clusters found was limited. 


\section{INTRODUCTION}

Several concepts have been developed to describe the process of behavioural change (De Vries et al., 2003, Prochaska and DiClemente, 1983, Schwarzer, 2004, Weinstein and Sandman, 2002), of which the stages of change concept proposed by Prochaska and colleagues as part of the Transtheoretical Model (TTM) (Fava et al., 1995, Prochaska and DiClemente, 1983, Prochaska et al., 1997) is the most widely known and used. For the present study, the I-Change model was used as a theoretical framework. This model states that the intention to change behaviour is determined by predisposing factors (behavioural, psychological, biological, social cultural factors), awareness factors (knowledge, cues to action, risk perception) and motivational factors (attitude, social influence and self-efficacy). The intention to change in combination with an individual's abilities and experienced barriers subsequently influences the likelihood of accomplishing the desired behaviour change. In line with the TTM, the I-Change model (De Vries et al., 2003) argues that behavioural change is a process that unfolds overtime through a sequence of five stages (De Vries et al., 2003). The first three stages are defined by the time an individual expects to elapse between the present moment and the intended behaviour change. These pre-action stages are precontemplation (not intending to quit within 6 months), contemplation (intending to quit within 6 months) and preparation (intending to quit within one month). After moving through the action stage (has quit for $<6$ months), the ex-smoker can progress to maintenance (has quit for $>6$ months) or relapse to smoking (De Vries et al., 2003). As we used the operationalizations proposed by the I-Change model (De Vries et al., 2003) and as it has recently received multiple critiques (Sutton, 2001, West, 2005), the criterion that smokers in preparation should have attempted to quit in the last year was abandoned in the present study. Contemplators were considered those smokers intending to quit within the next 6 months, regardless of previous quit attempts. The stages of change concept assumes that people within the same stage face common barriers to change, whereas people in different stages face different barriers (Weinstein et al., 1998). Therefore, if progress through the stages is to occur, health educators should adapt their messages to those determinants of behaviour change relevant to the stage an individual is in (Norman et al., 2000, Weinstein et al., 1998).

Stage-based tailoring has been used as a basis for several smoking cessation programmes (Erol and Erdogan, 2008, Meyer et al., 2008), as well as for interventions targeting other health behaviours (Johnson et al., 2006, Johnson et al., 2008). Over time, doubts have arisen regarding the viability of the stages of change as the underlying conceptualization of health promotion interventions (de Vet et al., 2007, Heather, 1991, Littel and Girvin, 2002, Sutton, 2001). Stage-based interventions are based on the assumption that smokers in the same stage benefit from targeting the same psychological constructs (Dijkstra et al., 2006, Prochaska et al., 2008). However, a recent review by Herzog (Herzog, 2008) suggest that contemplation consists of a mixture of smokers who are moderate to high in motivation to quit, causing 
within-stage heterogeneity. This can be a possible indication of the stages not being mutually exclusive but also of the contemplation stage of change being too comprehensive. A closer look should be taken at this stage to investigate whether it encompasses potential subtypes.

The idea that subtypes exist within stages has received considerable attention in recent studies (Anatchkova et al., 2005, Anatchkova et al., 2006a, Anatchkova et al., 2006b, Norman et al., 2000, Velicer et al., 1995, de Vries et al., 2008, Dijkstra and De Vries, 2000). However, only three of these studies have focused on the contemplation stage (Anatchkova et al., 2005, Norman et al., 2000, Velicer et al., 1995). In all three studies, four subtypes within contemplation were identified: the Classic Contemplator (with above average scores on both the pros and cons of smoking, resulting in a cognitive conflict, and high scores on the perceived temptations to smoke), the Early Contemplator (with a profile corresponding to precontemplation; scoring higher on the pros than on the cons of smoking and perceiving average temptations to smoke), the Progressing contemplator (with a profile corresponding to preparation; with the cons outweighing the pros of smoking and still perceiving average temptations to smoke) and a subtype demonstrating a lack of concern regarding both pros and cons and situational temptations, labelled Disengaged (scoring slightly below or above average on all scales). However, all studies clustered respondents based on the pros and cons of smoking and perceived temptations to smoke.

The current study is the first that addresses the replication of clusters within contemplation in a non-American sample. In addition, it is the first that uses cluster analysis based on the scores on three cognitive clustering variables directly related to cessation. On the basis of theoretical and empirical considerations (Dijkstra et al., 1996, Prochaska et al., 1991, Prochaska et al., 1994) and on studies finding an inverse relationship between self-efficacy and temptation (Diclemente et al., 1985, Rabois and Haaga, 2003), the pros of quitting, the cons of quitting and self-efficacy to quit are treated as being complementary to the cons of smoking, pros of smoking and temptation, respectively. This assumption of complementarities between clustering variables used in this and previous studies (Anatchkova et al., 2005, Norman et al., 2000, Velicer et al., 1995) makes it possible to compare the present study with earlier studies. However, according to the compatibility principle of Ajzen (Ajzen, 1988), predictors of a target behaviour should be measured in a similar manner as the target behaviour itself. As smoking cessation interventions aim at moving people toward quitting smoking, determinants of behaviour such as attitude and selfefficacy should also be measured with respect to quitting, not smoking. In correspondence with this principle, we expect that the use of clustering variables directly related to quitting will provide a more accurate cluster solution. 


\section{METHODS}

\section{Participants}

Smokers were recruited to take part in a randomized controlled trial (RCT) investigating the effectiveness of a computer-tailored smoking cessation programme in Dutch general practices. Seventy-five general practices applied a passive recruitment method (posters were displayed in the waiting area and questionnaires were provided in a separate folder stand, leaving uptake to the smoker's initiative) during 8 months and recruited 665 adult smokers (aged $>18$ years). Of these respondents, $84(12.5 \%)$ were in precontemplation, 218 (32.4\%) in contemplation, 265 $(39.4 \%)$ in preparation and $102(15.2 \%)$ were so called immotives [33]. Twentyfour respondents had missing values on any of the cluster variables and were therefore excluded from analyses, leaving a sample of 194 contemplators eligible for the present study. One hundred and forty-nine (76.8\%) completed the 6-month followup questionnaire. As no main effect of the intervention was found neither on 7-day point prevalence abstinence nor on continuous abstinence (having refrained from smoking since the previous measurement point), between conditions in the RCT (Hoving et al., 2010), both respondents in the experimental group and in the control group were included in the present study. Full details about the recruitment and sampling procedure can be found in Hoving et al. (Hoving et al., 2007a).

\section{Baseline measurement}

Baseline characteristics were collected using a written questionnaire consisting of 54 questions based on the I-Change model (De Vries et al., 2003). This questionnaire has been tested experimentally among Dutch smoking adults in previous studies (Dijkstra et al., 1996, Dijkstra et al., 1998a, Hoving et al., 2007a). Variables relevant to the present study included demographics, addiction level, the occurrence of cardiovascular and respiratory diseases, attitude, self-efficacy and the number of previous quit attempts, along with stage of change as a measure of the smoker's intention to quit.

Education Education was measured with one item, recoded into three categories: 'low' (1)=primary school/basic vocational school; 'medium' (2)=secondary vocational school/high school degree and 'high' (3)=higher vocational school/college degree/university degree.

Addiction level Addiction level was measured with three items: the tobacco product smoked, the amount of tobacco smoked daily (in number of cigarettes) and the time that elapses between getting out of bed and smoking $(<5 \mathrm{~min}$, between 6 and $30 \mathrm{~min}, 31$ to $60 \mathrm{~min}$ and $>60 \mathrm{~min}$ ). Based on these three items, an addiction score was calculated and respondents were classified based on the abbreviated Fagerström scale (Heatherton et al., 1991), varying from 0 (not addicted) to 7 (highly addicted). 
The occurrence of cardiovascular and respiratory diseases The occurrence of cardiovascular and respiratory diseases was measured by one dichotomous item each, asking whether the respondent suffered from this disease ( $0=$ no; $1=y e s)$.

Number of previous quit attempts The number of previous quit attempts was assessed with one item, asking the respondents how often they had tried to quit smoking in the past.

Pros of quitting Pros of quitting were measured by 11 items. Statements were given with which a respondent could agree or disagree, measured on a 4-point Likert scale $(0=$ disagree/do not know; 1 =somewhat agree; $2=$ agree; $3=$ fully agree; e.g. 'When I quit smoking, my health will improve'; a=0.84).

Cons of quitting Cons of quitting were measured by 5 items. Statements were given with which a respondent could agree or disagree, measured on a 4-point Likert scale ( 0 =disagree/do not know; 1 =somewhat agree; $2=$ agree; $3=$ fully agree; e.g. 'When I quit smoking, I will gain weight'; $a=0.53$ ). This is comparable to the internal consistency of the cons-of-quitting subscale found in previous studies (a=0.57) (Dijkstra et al., 1996, Dijkstra et al., 1998a).

Self-efficacy Self-efficacy was measured by 15 items (-2 =disagree; 2=agree) asking respondents to indicate whether they would be able to refrain from smoking in various situations (e.g. when being angry, when being offered a cigarette; $a=0.90$ ).

Stage of change Stage of change was measured with one item, asking respondents to state within what time span they intended to quit smoking on a 7-point scale (within 1 month, within 6 months, within 1 year, within 5 years, at some point but not within 5 years, never quit but smoke less or use tobacco products with lower nicotine and tar levels or never quit nor smoke less nor use tobacco products with lower nicotine and tar levels). With regard to preparation, a slightly different definition was used than originally defined by the TTM (DiClemente et al., 1991). In the TTM, preparation is defined as intending to quit within 1 month while having attempted to quit in the last year. However, according to this definition, contemplators who have never quit smoking cannot move to preparation but will have to progress to action directly. Additionally, by including the criterion of having made a quit attempt in the last year, the stages of change paradigm does not solely measure intention to change behaviour but also assesses actual behaviour change. Using the operationalizations proposed by the I-Change model (De Vries et al., 2003), this criterion was abandoned in the present study. As only smokers motivated to quit within 6 months, but not within 1 month, were included, smokers in preparation were excluded.

\section{Follow-up measurement}

Six months after baseline, respondents were contacted by telephone and asked about their smoking status and their smoking activity since baseline.

Four outcome measures were assessed: '7-day point prevalence abstinence' (having refrained from smoking during the last 7 days; $0=$ no; $1=y e s$ ), 'continued abstinence' (having refrained from smoking since the previous measurement point; $0=$ no; 
$1=y e s$ ) and 'having made one or more serious quit attempts (at least 24 hours of abstinence) since the previous measurement point' ( $0=$ no; $1=y e s)$.

Stage of change was measured again to determine whether the respondent had moved through the stages since baseline. 'Stage transition' was defined as negative (-1), neutral (0) or positive (+1) (Dijkstra et al., 1998b).

\section{Analysis}

Cluster analysis is a statistical method used to group individuals into clusters in such a manner that individuals within one class are similar to each other with regard to specific variables, but unlike individuals in other classes (Everitt, 1980, Field, 2000). Clustering variables (pros and cons of quitting and self-efficacy) were measured at baseline and were standardized to z-scores. The squared Euclidian distance metric and Ward's minimum variance clustering algorithm were used as distance metric and clustering algorithm, respectively. Ward's method has been shown to be one of the more reliable methods within cluster analysis and has been especially widely used in the behavioural sciences (Borgen and Barnett, 1987). The squared Euclidian distance is the proximity measure it is most often applied with and reflects the level, profile shape and scatter of the cluster solution (compared with correlation measures, which only reflect the shape and scatter) (Borgen and Barnett, 1987).

Multiple methods were used to determine the number of clusters (Everitt, 1980, Norman et al., 2000). First, inverse scree tests were conducted to provide an indication of the optimal number of clusters. Second, cluster profiles were visually inspected to determine their interpretability. Attention was paid to the shape, level and scatter of the separate cluster profiles (Cronbach and Gleser, 1953). Third, the stability of the cluster solutions was examined with non-hierarchical cluster analyses within random subsamples, each independently drawn from the total sample and consisting of $50 \%$ of respondents.

To cross-sectionally compare the cluster solutions found, one-way analyses of variance (ANOVA) with Tukey's Honestly Significant Difference (HSD) ad hoc comparisons were conducted to compare clusters for age, addiction level and the number of previous quit attempts. Chi-square tests were used to compare clusters regarding gender, educational level and suffering from a cardiovascular and/or respiratory disease. Furthermore, logistic regression analysis was used to test whether 7-day point prevalence abstinence, continued abstinence and having made one or more serious quit attempts since the previous measurement point could be predicted by cluster membership at baseline. Comparisons were made first with the cluster of Early Contemplators and second with the cluster of Progressing Contemplators as a reference category. In addition, multinomial logistic regression analysis was used to determine whether cluster membership had any predictive value with regard to stage transition (reference category=neutral). For longitudinal validation of the clusters, missing values were replaced to avoid misleading artefacts because of dropout. 
A sensitively analysis was conducted, consisting of three different analyses. Both a negative scenario (respondents with missing values at 6 months follow-up were considered as smokers) and a positive scenario (respondents with missing values at 6 months follow-up were considered as non-smokers) were investigated and a complete-case analysis was conducted. Data were analyzed using SPSS 15.0.

\section{RESULTS}

\section{Sample characteristics}

The age of respondents included in the analyses varied from 18 to 79 years (mean=40.4; SD=12.1). Women were slightly overrepresented (61.1\%) and most respondents reported a medium level of education (secondary vocational school or high school; 55.6\%). A description of the overall sample and respondents' scores on the cognitive variables on which cluster analysis was based are presented in Table 10.1.

Table 10.1 A description of the overall sample at baseline ( $\mathrm{N}=194)$

\begin{tabular}{lc}
\hline Variables & \\
\hline $\begin{array}{l}\text { Demographics } \\
\text { Gender } \\
\quad \text { Male (\%) }\end{array}$ & 38.9 \\
Age (Mean; S.D.) & $40.4 ; 12.1$ \\
Educational level & 20.4 \\
$\quad$ Low (\%) & 55.6 \\
$\quad$ Medium (\%) & 24.1 \\
$\quad$ High (\%) & $3.3 ; 1.5$ \\
Addiction level (Mean; S.D.) ${ }^{\mathrm{a}}$ & 4.2 \\
Cardiovascular disease & \\
$\quad$ Yes (\%) & 20.8 \\
$\quad$ Respiratory disease & \\
$\quad$ Yes (\%) & $1.96 ; .60$ \\
Cognitive variables & $1.24 ; .61$ \\
Pros of quitting (Mean; S.D.) & \\
Cons of quitting (Mean; S.D.) & $-.25 ; .78$ \\
Self-efficacy to quit (Mean; S.D.) & \\
Smoking related behaviour & $2.5 ; 2.9$ \\
Number of previous quit attempts (Mean; S.D.) & \\
\hline
\end{tabular}

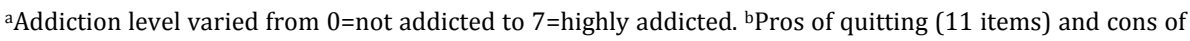
quitting ( 5 items) were measured on a 4-point Likert scale ( $0=$ disagree/do not know; $1=$ somewhat agree; $2=$ agree; $3=$ fully agree). cSelf-efficacy to quit (15 items) was measured on a 5-point Likert scale ($2=$ disagree; 2 =agree).

\section{Cluster analysis}

Inverse scree tests and examining the stability of different cluster solutions indicated that a three-cluster solution best represented the data. However, as a fourcluster solution was consistently found in previous studies (Anatchkova et al., 2005, 
Everitt, 1980, Norman et al., 2000, Velicer et al., 1995), we investigated both cluster solutions with regard to their interpretability.

For both cluster solutions, clusters differed significantly from each other with regard to pros and cons of quitting and self-efficacy to quit $(\mathrm{P}<0.001)$. The threecluster solution, though, replicated better across subsamples than the four cluster solution. Cluster means and standard deviations are presented in Tables 10.2 and 10.3 for the three-cluster solution and the four-cluster solution, respectively. Figures 10.1 and 10.2 display the cluster profiles for the three and four clusters identified.

The investigation of the cluster solutions' interpretability indicated a threecluster solution over and above four clusters. Therefore, the following section only elaborates on the three clusters found.

Cluster 1 ( $N=46 ; 23.7 \%)$ The first cluster was labelled Progressing Contemplation. Smokers in this cluster scored a standard deviation above average on the pros of quitting, average on the cons of quitting and scored half a standard deviation above average on self-efficacy. For the Progressing Contemplator, pros of quitting outweighed the cons, whereas self-efficacy scores were relatively high.

Cluster 2 ( $N=53 ; 27.3 \%)$ The second cluster was labelled Early Contemplation. Smokers in this cluster scored slightly above the overall sample's average on the pros of quitting, more than a standard deviation above average on the cons of quitting and more than a standard deviation below average on self-efficacy. For Early Contemplators, the cons of quitting outweighed the pros and self-efficacy perceptions were low.

Cluster 3 ( $N=95$; 49\%) Smokers in the third cluster were labelled Disengaged Contemplators. The profile of the disengaged smoker was characterized by about half a standard deviation below average scores for both the pros and cons of quitting and average scores on self-efficacy. 
2

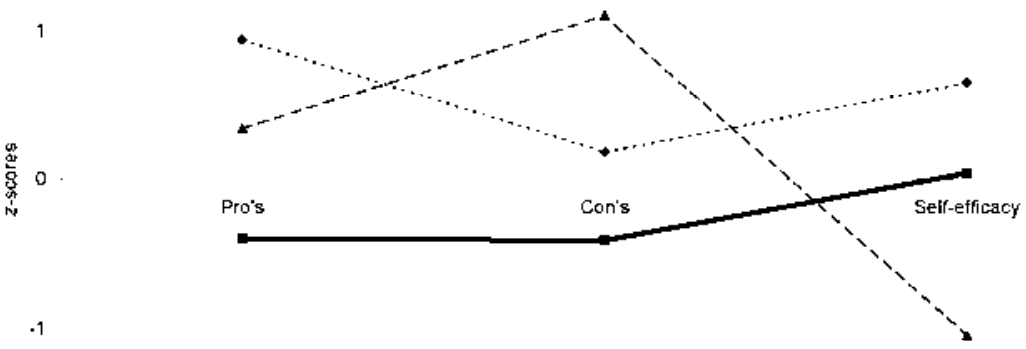

.2

\section{Variaptes}

- - Prggressing $(\mathrm{N}=46) \quad-$ Disengaged $(\mathrm{N}=95) \quad-*-$ Early (N)=53)

Figure 10.1 Cluster profiles for the three clusters identified within the three-cluster solution.

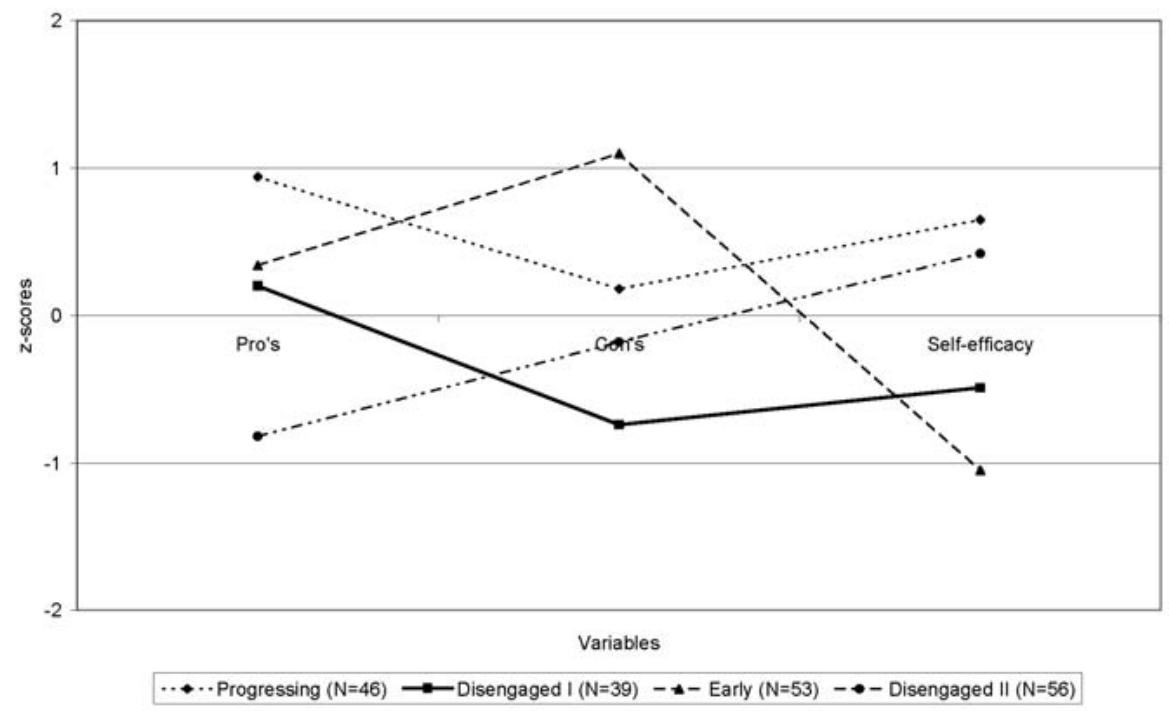

Figure 10.2 Cluster profiles for the four clusters identified within the four-cluster solution. 
Table 10.2 Cognitive clustering variables in standardized z-scores (mean=0; $S D=1)$ for the three clusters found

\begin{tabular}{|c|c|c|c|c|}
\hline Total sample & $\begin{array}{c}\text { 1. Early } \\
(\mathrm{N}=53) \\
\text { Mean; S.D. }\end{array}$ & $\begin{array}{l}\text { 2. Progressing } \\
(\mathrm{N}=46) \\
\text { Mean; S.D. }\end{array}$ & $\begin{array}{l}\text { 3. Disengaged } \\
(\mathrm{N}=95) \\
\text { Mean; S.D. }\end{array}$ & Tukey HSD Pattern \\
\hline Pros of quitting & $.34 ; .83$ & $.94 ; .42$ & $-.40 ; .86$ & $3<1<2 * * *$ \\
\hline Cons of quitting & $1.10 ; .64$ & $.18 ; .72$ & $-.41 ; .72$ & $3<2<1 * * *$ \\
\hline Self-efficacy to quit & $-1.05 ; .47$ & $.65 ; .81$ & $.05 ; .780$ & $1<3<2^{* * *}$ \\
\hline Random subsample 1 & $\begin{array}{l}\text { 1. Early } \\
(\mathrm{N}=34)\end{array}$ & $\begin{array}{l}\text { 2. Progressing } \\
\qquad(\mathrm{N}=41)\end{array}$ & $\begin{array}{l}\text { 3. Disengaged } \\
(\mathrm{N}=29)\end{array}$ & \\
\hline Pros of quitting & $.70 ; .60$ & $.49 ; .72$ & $-.87 ; .63$ & $3<1,2^{*}$ \\
\hline Cons of quitting & $1.13 ; .62$ & $-.11 ; .69$ & $-.40 ; .68$ & $3<2<1 *$ \\
\hline Self-efficacy to quit & $-.58 ; .58$ & $.53 ; 1.01$ & $-.40 ; .83$ & $1<3<2 * *$ \\
\hline Random subsample 2 & $\begin{array}{l}\text { 1. Early } \\
(\mathrm{N}=24)\end{array}$ & $\begin{array}{l}\text { 2. Progressing } \\
\qquad(\mathrm{N}=25)\end{array}$ & $\begin{array}{l}\text { 3. Disengaged } \\
(\mathrm{N}=59)\end{array}$ & \\
\hline Pros of quitting & $1.03 ; .52$ & $.96 ; .32$ & $-.44 ; .77$ & $3<2^{* * *} ; 3<1^{* * *}$ \\
\hline Cons of quitting & $1.28 ; .67$ & $-.52 ; .62$ & $-.00 ; .81$ & $2<1^{* * *} ; 3<1^{* * *}$ \\
\hline Self-efficacy to quit & $-1.10 ; .57$ & $.66 ; 95$ & $-.00 ; .80$ & $3<2^{* * *} ; 1<2^{* * *}$ \\
\hline Random subsample 3 & $\begin{array}{l}\text { 1. Early } \\
(\mathrm{N}=25)\end{array}$ & $\begin{array}{l}\text { 2. Progressing } \\
(\mathrm{N}=29)\end{array}$ & $\begin{array}{l}\text { 3. Disengaged } \\
(\mathrm{N}=52)\end{array}$ & \\
\hline Pros of quitting & $.16 ; .91$ & $.92 ; .40$ & $-.45 ; .79$ & $3<1<2 * *$ \\
\hline Cons of quitting & $1.13 ; .51$ & $.18 ; .71$ & $-.46 ; .73$ & $3<2<1^{* * *}$ \\
\hline Self-efficacy to quit & $-1.07 ; .53$ & $.65 ; .68$ & $-.02 ; 83$ & $1<3<2 * * *$ \\
\hline
\end{tabular}

HSD, honestly significant difference; ${ }^{*} \mathrm{P}<0.05$; ${ }^{* *} \mathrm{P}<0.01$; ${ }^{* * *} \mathrm{P}<0.001$. 
Table 10.3 Cognitive clustering variables in standardized z-scores (mean=0; $S D=1$ ) for a four-cluster solution

\begin{tabular}{|c|c|c|c|c|c|}
\hline Total sample & $\begin{array}{c}\text { 1. Early } \\
(\mathrm{N}=53) \\
\text { Mean; S.D. }\end{array}$ & $\begin{array}{l}\text { 2. Progressing } \\
\qquad(\mathrm{N}=46) \\
\text { Mean; S.D. }\end{array}$ & $\begin{array}{l}\text { 3. Disengaged I } \\
(\mathrm{N}=39) \\
\text { Mean; S.D. }\end{array}$ & $\begin{array}{l}\text { 4. Disengaged II } \\
(\mathrm{N}=56)\end{array}$ & $\begin{array}{l}\text { Tukey HSD } \\
\text { Pattern }\end{array}$ \\
\hline Pros of quitting & $.34 ; .83$ & $.94 ; .42$ & $.20 ; .75$ & $-.82 ; .67$ & $4<1,3<2^{* * *}$ \\
\hline Cons of quitting & $1.10 ; .64$ & $.18 ; .72$ & $-.74 ; .56$ & $-.18 ; .73$ & $3<4<2<1^{* *}$ \\
\hline Self-efficacy to quit & $-1.05 ; .47$ & $.65 ; .81$ & $-.49 ; .56$ & $-.42 ; .69$ & $1<3<2,4^{* * *}$ \\
\hline Random subsample 1 & $\begin{array}{l}\text { 1. Early } \\
(\mathrm{N}=28)\end{array}$ & $\begin{array}{l}\text { 2. Progressing } \\
\qquad(\mathrm{N}=27)\end{array}$ & $\begin{array}{l}\text { 3. Disengaged I } \\
(\mathrm{N}=16)\end{array}$ & $\begin{array}{l}\text { 4. Disengaged II } \\
\qquad(\mathrm{N}=28)\end{array}$ & \\
\hline Pros of quitting & $.41 ; .74$ & $.60 ; .62$ & $-.75 ; .52$ & $-1.01 ; .62$ & $4<3,1,2 * * *$ \\
\hline Cons of quitting & $.90 ; .59$ & $.09 ; .59$ & $-.43 ; .42$ & $-.48 ; .66$ & $4,3,2<1^{*}$ \\
\hline Self-efficacy to quit & $-.90 ; .49$ & $.72 ; .46$ & $-.52 ; .40$ & $-.09 ; .89$ & $\begin{array}{c}1,3,4<2^{* * *} \\
1<4^{* * *}\end{array}$ \\
\hline Random subsample 2 & $\begin{array}{l}\text { 1. Early } \\
(\mathrm{N}=19)\end{array}$ & $\begin{array}{l}\text { 2. Progressing } \\
\qquad(\mathrm{N}=36)\end{array}$ & $\begin{array}{l}\text { 3. Disengaged I } \\
\qquad(\mathrm{N}=30)\end{array}$ & $\begin{array}{l}\text { 4. Disengaged II } \\
\qquad(\mathrm{N}=20)\end{array}$ & \\
\hline Pros of quitting & $.79 ; .81$ & $.40 ; .60$ & $-.14 ; .81$ & $-1.11 ; .82$ & $4<3<2^{* * *} ; 3<1^{*}$ \\
\hline Cons of quitting & $1.28 ; .76$ & $.33 ; .51$ & $-.67 ; .67$ & $-.44 ; .68$ & $3,4<2<1^{* * *}$ \\
\hline Self-efficacy to quit & $-1.04 ; .41$ & $-.18 ; 52$ & $.86 ; .52$ & $-.75 ; .66$ & $4,1<3<2 * *$ \\
\hline Random subsample 3 & $\begin{array}{l}\text { 1. Early } \\
(\mathrm{N}=10)\end{array}$ & $\begin{array}{l}\text { 2. Progressing } \\
\qquad(\mathrm{N}=21)\end{array}$ & $\begin{array}{l}\text { 3. Disengaged I } \\
\qquad(\mathrm{N}=28)\end{array}$ & $\begin{array}{l}\text { 4. Disengaged II } \\
\qquad(\mathrm{N}=25)\end{array}$ & \\
\hline Pros of quitting & $.29 ; .82$ & $.74 ; .60$ & $.72 ; .68$ & $-1.15 ; .49$ & $4<3,1,2^{* *}$ \\
\hline Cons of quitting & $1.66 ; .43$ & $-.49 ; .60$ & $.36 ; .53$ & $-.34 ; .73$ & $4,2<3<1^{* * *}$ \\
\hline Self-efficacy to quit & $-1.05 ; .48$ & $1.00 ; .84$ & $-.32 ; 52$ & $.05 ; .77$ & $1<3^{*} ; 1<4<2^{* * *}$ \\
\hline
\end{tabular}

HSD, honestly significant difference. ${ }^{*} \mathrm{P}<0.05 ;{ }^{* *} \mathrm{P}<0.01 ;{ }^{* * *} \mathrm{P}<0.001$.

\section{Validation}

Table 10.4 displays the results from the cross-sectional comparison of the clusters. A significant difference between the Early Contemplation cluster and the Disengaged cluster was found for addiction level ( $\mathrm{F}=9.338 ; \mathrm{df}=2 ; \mathrm{P}<0.001)$. Disengaged smokers scored significantly lower on addiction level compared with Early Contemplating smokers. Therefore, regarding these two clusters, addiction level was taken into account as a confounder in regression analyses determining the predictive validity of the clusters on behavioural outcomes. As is shown in Tables 10.5 and 10.6, cluster membership was not predictive of 7-day point prevalence abstinence, continued abstinence, having made one or more serious quit attempts since the previous measurement or stage transition. Moreover, conducting effect analyses for each subtype separately using condition 3 subtype interaction terms did not yield any significant differences between the experimental and control group for any of the clusters separately. Results reported are based on a negative scenario, in which respondents lost to follow-up were considered as smokers. When repeating the 
analyses with a positive scenario or with complete cases only, results did not change (results not reported).

Table 10.4 Cross-sectional comparison of the cluster solution: comparing clusters in terms of items concerning demographics, addiction level, the occurrence of cardiovascular and respiratory diseases and the number of previous quit attempts

\begin{tabular}{|c|c|c|c|c|c|c|}
\hline & $\begin{array}{l}\text { 1. Early } \\
(\mathrm{N}=53)\end{array}$ & $\begin{array}{l}\text { 2. Progressing } \\
\qquad(\mathrm{N}=46)\end{array}$ & $\begin{array}{l}\text { 3. Disengaged } \\
(\mathrm{N}=95)\end{array}$ & $\begin{array}{c}\text { Chi-square } \\
\text { (df) }\end{array}$ & $F(d f)$ & $\begin{array}{c}\text { Tukey HSD } \\
\text { pattern }\end{array}$ \\
\hline \multicolumn{7}{|l|}{ Variables } \\
\hline Gender ( $\%$ male) & 32.1 & 41.3 & 41.5 & $1.42(2)$ & & \\
\hline Age (Mean; S.D.) & $38.81 ; 10.43$ & $39.15 ; 11.48$ & $41.96 ; 13.09$ & & $1.50(2)$ & ns \\
\hline Educational level & & & & $4.90(4)$ & & \\
\hline Low (\%) & 29.2 & 11.8 & 18.8 & & . & . \\
\hline Medium (\%) & 54.2 & 58.8 & 55.0 & & . & . \\
\hline High (\%) & 16.7 & 29.4 & 26.3 & & . & . \\
\hline Addiction level (Mean; S.D.) & $3.96 ; 1.55$ & $3.44 ; 1.21$ & $2.93 ; 1.56$ & . & $8.34(2)$ & $1>3^{*}$ \\
\hline $\begin{array}{l}\text { Cardiovascular disease (\% } \\
\text { yes) }\end{array}$ & 5.7 & - & 5.3 & $2.50(2)$ & & \\
\hline Respiratory disease (\% yes) & 26.4 & 15.9 & 20.0 & $1.69(2)$ & & \\
\hline $\begin{array}{l}\text { Number of previous quit } \\
\text { attempts (Mean; S.D.) }\end{array}$ & $3.21 ; 3.88$ & $2.26 ; 1.72$ & $2.18 ; 2.66$ & . & $2.34(2)$ & ns \\
\hline
\end{tabular}

HSD, honestly significant difference. na, Not applicable; ns, non-significant; ${ }^{*} \mathrm{P}<0.05 ;{ }^{* *} \mathrm{P}<0.01 ;{ }^{* * *} \mathrm{P}<$ 0.001 . 
Table 10.5 Predictive validity of contemplation subtypes with regard to three measures of smoking behaviour change

\begin{tabular}{|c|c|c|c|c|c|c|c|c|c|c|c|c|}
\hline & \multicolumn{4}{|c|}{$\begin{array}{l}\text { 7-day point prevalence } \\
\text { abstinence }\end{array}$} & \multicolumn{4}{|c|}{ continued abstinence } & \multicolumn{4}{|c|}{ 24-hour quit attempt } \\
\hline & \multirow[t]{2}{*}{ OR } & \multirow[t]{2}{*}{$R^{2}$} & \multicolumn{2}{|l|}{$95 \% \mathrm{CI}$} & \multirow[t]{2}{*}{ OR } & \multirow[t]{2}{*}{$R^{2}$} & \multicolumn{2}{|l|}{$95 \% \mathrm{CI}$} & \multirow[t]{2}{*}{ OR } & \multirow[t]{2}{*}{$R^{2}$} & \multicolumn{2}{|l|}{$95 \% \mathrm{CI}$} \\
\hline & & & Lower & Upper & & & Lower & Upper & & & Lower & Upper \\
\hline \multicolumn{13}{|c|}{ Reference category: Early Contemplators } \\
\hline Step 1 & \multicolumn{3}{|c|}{.022} & & \multicolumn{3}{|c|}{.025} & & \multicolumn{3}{|c|}{.005} & \\
\hline Progressing & 2.15 & & .59 & 7.90 & 3.05 & & .56 & 16.54 & .69 & & .27 & 1.72 \\
\hline Disengaged & 2.31 & & .72 & 7.36 & 2.35 & & .48 & 11.52 & .86 & & .40 & 1.83 \\
\hline \multicolumn{13}{|l|}{ Step 2} \\
\hline Progressing & 2.06 & & .56 & 7.58 & 3.66 & & .65 & 20.48 & 63 & & .25 & 1.61 \\
\hline Disengaged & 2.09 & & .63 & 6.90 & 3.15 & & .61 & 16.34 & .73 & & .33 & 1.62 \\
\hline Addiction level & .91 & & .68 & 1.20 & 1.32 & & .89 & 1.96 & .86 & & .69 & 1.07 \\
\hline \multicolumn{13}{|c|}{ Reference category: Progressing Contemplators } \\
\hline Step 1 & \multicolumn{3}{|c|}{.022} & & \multicolumn{3}{|c|}{.025} & & \multicolumn{3}{|c|}{.005} & \\
\hline Disengaged & 1.07 & & .40 & 2.84 & .77 & & .24 & 2.51 & 1.25 & & .54 & 2.90 \\
\hline Early & .46 & & .13 & 1.70 & .33 & & .06 & 1.78 & 1.46 & & .58 & 3.67 \\
\hline \multicolumn{13}{|l|}{ Step 2} \\
\hline Disengaged & 1.02 & & .38 & 2.73 & .86 & & .26 & 2.83 & 1.16 & & .49 & 2.71 \\
\hline Early & .49 & & .13 & 1.80 & .27 & & .05 & 1.53 & 1.58 & & .62 & 4.00 \\
\hline Addiction level & .91 & & .68 & 1.20 & 1.32 & & .89 & 1.96 & .86 & & .69 & 1.07 \\
\hline
\end{tabular}

${ }^{*} \mathrm{P}<0.05 ;{ }^{* *} \mathrm{P}<0.01 ;{ }^{* * *} \mathrm{P}<0.001$.

Table 10.6 Predictive validity of contemplation subtypes with regard to transition through the stages of change

\begin{tabular}{|c|c|c|c|c|c|c|c|c|}
\hline & \multicolumn{4}{|c|}{$\begin{array}{l}\text { Positive stage transition } \\
(\mathrm{N}=55)\end{array}$} & \multicolumn{4}{|c|}{$\begin{array}{l}\text { Negative stage transition } \\
(\mathrm{N}=28)\end{array}$} \\
\hline & \multirow[t]{2}{*}{ OR } & \multirow[t]{2}{*}{$R^{2}$} & \multicolumn{2}{|l|}{$95 \%$ CI } & \multirow[t]{2}{*}{ OR } & \multirow[t]{2}{*}{$R^{2}$} & \multicolumn{2}{|l|}{$95 \% \mathrm{CI}$} \\
\hline & & & Lower & Upper & & & Lower & Upper \\
\hline Early vs. Disengaged & 1.28 & .008 & .59 & 2.77 & 1.60 & .008 & .62 & 4.10 \\
\hline Early vs. Disengaged & 1.57 & & .68 & 3.59 & 1.83 & & .67 & 5.00 \\
\hline Addiction level & .83 & & .65 & 1.06 & .88 & & .65 & 1.19 \\
\hline Early vs. Progressing & 1.28 & .018 & .52 & 3.15 & 2.07 & .018 & .63 & 6.86 \\
\hline Early vs. Progressing & 1.39 & & .55 & 3.51 & 2.14 & & .63 & 7.26 \\
\hline Addiction level & .91 & & .65 & 1.25 & 1.01 & & .67 & 1.53 \\
\hline Disengaged vs. Progressing & 1.00 & .002 & .45 & 2.24 & .77 & .002 & .25 & 2.37 \\
\hline Disengaged vs. Progressing & 1.02 & & .45 & 2.33 & .74 & & .24 & 2.30 \\
\hline Addiction level & .90 & & .69 & 1.17 & 1.02 & & .72 & 1.45 \\
\hline
\end{tabular}

Note: reference category is neutral (no transition) $(\mathrm{N}=111){ }^{*} \mathrm{p}<0.05$; $^{* *} \mathrm{p}<0.01$; $^{* *} \mathrm{p}<0.001$. 


\section{DISCUSSION}

\section{Main conclusions}

The present study suggests that subtypes exist within the contemplation stage of change, which are comparable to those found in previous studies (Anatchkova et al., 2005, Everitt, 1980, Norman et al., 2000, Velicer et al., 1995). Three of four clusters could be replicated: Early, Progressing and Disengaged Contemplators. The Classic Contemplator as found previously (Anatchkova et al., 2005, Everitt, 1980, Norman et al., 2000, Velicer et al., 1995) was not found within the present sample. As this cluster could not be identified, doubts may arise whether the typical contemplator as suggested (Prochaska et al., 1997, Velicer et al., 1999) truly exists. One possible explanation for not finding the Classic Contemplator might be that the variables used for cluster analysis were directly related to smoking cessation (i.e. the desired behaviour), while previous studies used the pros and cons of smoking (i.e. the undesired behaviour). However, as longitudinal validation of the clusters was limited, support for the cluster solution found is not extremely strong yet. Additional support for our three-cluster solution was obtained by cross-sectionally comparing the clusters. Disengaged smokers were significantly less dependent on nicotine than Early Contemplators. This finding corresponds with previous findings indicating that the Disengaged smoker seems to be a light smoker (Anatchkova et al., 2005, Norman et al., 2000). Furthermore, the high degree of dependence among Early Contemplators in comparison with Disengaged smokers potentially explains why the former reported a lower score on self-efficacy. A previous study showed that the more dependent on nicotine a smoker is, the lower self-efficacy to quit and consequently the higher the risk of relapsing (Ockene et al., 2000). For the three clusters found, traditional interventions for contemplators might be inappropriate. Interventions targeting smokers in contemplation typically attempt to solve the cognitive conflict contemplators are said to experience (Anatchkova et al., 2005, Prochaska et al., 1997, Prochaska et al., 2005, Velicer et al., 1999) by aiming at decreasing the perceived cons of quitting (Dijkstra et al., 2006). However, in our sample no contemplators were in such a cognitive conflict about their smoking behaviour. Only the Disengaged cluster showed a similar score on both pros and cons of quitting, though both scores were below average. Also a significant lack of selfefficacy, characteristic for the Classic Contemplator, was not observed within this group of smokers. The Disengaged smoker thus might be able to change but is not interested in changing. These smokers might benefit most from an intervention aimed at raising the pros of quitting, typically designed for precontemplators (Dijkstra et al., 2006). As interventions aimed at contemplators typically focus on lowering the cons, the appropriateness of stage-based interventions may need to be re-evaluated. 


\section{Recommendations}

Based on the present study, several recommendations can be made for future research. First, no study to date has been able to fully understand the cluster of Disengaged Contemplators (Anatchkova et al., 2005, Norman et al., 2000, Velicer et al., 1995). Within our study, almost half of the respondents could be considered Disengaged. As this is probably the cluster of smokers most difficult to change, this highlights the complex nature of smokers said to be in contemplation (Hoving et al., 2006c, Velicer et al., 1999). Further research might need to start focusing more on Disengaged smokers. Perhaps these smokers would benefit from interventions focused on precontemplators, as these usually aim at increasing the perceived pros of quitting (Dijkstra et al., 2006). As they already perceive few cons of quitting and perceive a high self-efficacy to quit, they might then directly move to preparation. However, as no previous, quantitative studies have yet succeeded in explaining this cluster of smokers, qualitative research is vital to identify the variables that need to be addressed in smoking cessation interventions aimed at Disengaged smokers. Second, there is a need for more research to investigate whether the typical contemplator as suggested in previous studies does truly exist and consequently whether the strategy usually used in stage-based interventions targeting smokers contemplating to quit is still tenable. Third, together with prior studies focusing on subtypes within contemplation (Anatchkova et al., 2005, Norman et al., 2000, Velicer et al., 1995), this study can be considered the necessary first step in evaluating the viability of subtypes within contemplation. However, more work is required to evaluate the validity of this (sub) stage theory (Weinstein et al., 1998). Fourth, it has been suggested previously that preaction stages (precontemplation, contemplation and preparation) might not be qualitatively different (Herzog, 2008) and that a continuous measure of intention is perhaps a better predictor of behavioural change than the stages of change (de Vet et al., 2007). Future studies could investigate the relative predictive power of the subtypes found in this and other studies and compare these with continuous measures of motivation and readiness to change.

\section{Limitations}

This study is subject to certain limitations. First, the sample size was limited. This may have resulted in some non-significant results in the validation of the cluster solution. Moreover, the small sample size made sampling without replacement to examine the cluster solution's stability impossible. Therefore, we have used sampling with replacement, potentially somewhat subsiding our findings. Also due to the relatively small sample, missing values had to be replaced for longitudinal validation of clusters. Yet, conducting the same analyses replacing missing values with a negative and a positive outcome and conducting complete-case analyses revealed similar results. This indicates a certain robustness of our findings. However, repetition of the study with a larger sample is recommended. Second, as is inherent to 
every research, it is possible that a selection bias has occurred. However, we do not expect that such a bias had a large influence on our results, as the original study was presented as a study about smoking, not about quitting. We therefore do not expect that certain smoker types were not willing to participate. Indeed, as described earlier, precontemplators, contemplators, preparers as well as immotives took part in the study. Third, the reliability of the factor representing the cons of quitting was relatively low $(a=0.53)$. As this factor has been critical for the formation of subtypes, it may have somewhat influenced the results; thus, these have to be interpreted with caution. However, reliability was comparable in previous studies (Dijkstra et al., 1998a, Hoving, 2007, Hoving et al., 2007a). Fourth, we used a three-item measure of addiction level derived from the abbreviated Fagerström scale (Heatherton et al., 1991). Even though this abbreviated version has been well studied (Heatherton et al., 1991), the specific psychometric properties of the three-item measure are unknown. Fifth, based on previous work (De Vries et al., 1998, Dijkstra et al., 1996) and in line with many recent studies that have dropped the second criterion (Dijkstra et al., 1998d, Littel and Girvin, 2002, Sutton, 2001), we used a slightly different definition of the preparation stage of change than originally defined by the TTM (Prochaska et al., 1997). Smokers who reported to be willing to quit smoking within a month, whether or not they had attempted to quit in the last year, were defined as preparers and thus excluded in the present study. This decision might have somewhat limited the comparative value of this study, but as this criterion lately has received a lot of critique (Sutton, 2001, West, 2005), future studies within this field might consider using the same definition as has been used here. Sixth, cluster analysis is a heuristic procedure; the interpretation of the results may have depended on the researcher's perspective. Though, using multiple methods to determine the number of clusters has limited its influence (Everitt, 1980, Norman et al., 2000).

\section{Conclusions}

Our study supports the idea that subtypes exist within the contemplation stage of change, though the Classic Contemplator as identified previously could not be replicated within the present sample. However, as the population studied consisted of smokers contemplating to quit, clustering variables used here were compatible with the behaviour change aimed at (Ajzen, 1988). Therefore, it can be expected that the cluster solution presented in this paper was more accurate than those found in previous studies, using clustering variables related to smoking. Further research is necessary to confirm the subtypes found, which might provide an opportunity to further tailor smoking cessation interventions to the needs of groups of individuals with similar characteristics (Anatchkova et al., 2005, Anatchkova et al., 2006a, Anatchkova et al., 2006b, Dijkstra and De Vries, 2000, Norman et al., 2000, Velicer et al., 1995). 



\title{
CHAPTER 11
}

\author{
General Discussion
}


The main aim of the present dissertation was to develop and to evaluate the smoking cessation intervention PAS, consisting of an Internet-based multiple computer-tailored smoking cessation programme and tailored counselling by a practice nurse, with regard to its effectiveness, cost- effectiveness and feasibility. Additionally, as a positive motivation is a necessary though not always sufficient prerequisite for smoking cessation to occur, this dissertation aimed to inform the development of future smoking cessation interventions by contributing to an increased understanding of smokers' motivation to quit. The research questions this dissertation aimed to answer were therefore clustered around three main subjects, i.e. the evaluation of PAS, the dissemination of PAS and the construct of motivation:

\section{EVALUATION}

1. What is the effect of an Internet-based multiple computer-tailored smoking cessation programme on smoking abstinence when compared to a no intervention control group among a general population sample of smokers?

2. What is the effect of an Internet-based multiple computer-tailored smoking cessation programme combined with and without tailored counselling by a practice nurse on smoking abstinence when compared to care as usual among smokers recruited in a primary care setting?

3. What is the cost-effectiveness and cost-utility of an Internet-based multiple computer-tailored smoking cessation programme combined with and without tailored counselling by a practice nurse among smokers recruited in a primary care setting compared to care as usual and to each other?

\section{DISSEMINATION}

4. What are the determinants of practice nurses' intention to adopt a new smoking cessation intervention?

5. What are the determinants of practice nurses' intention to implement a new smoking cessation intervention?

6. What is the influence of recruitment strategy on the reach and effect of an Internet-based multiple tailored smoking cessation intervention amongst Dutch adult smokers?

\section{MOTIVATION}

7. What is the role of desire, duty and intention to quit smoking in predicting quit attempts?

8. Do subtypes exist within the contemplation stage of change?

This final chapter describes the main findings of the studies conducted with regard to each of these three subjects. It puts the findings into context with previously conducted research and describes their implications for theory, future research and practice. Finally this chapter discusses several methodological and practical consid- 
erations in light of which the results presented in this dissertation should be interpreted and provides a general conclusion.

\section{EVALUATION}

\section{Main findings}

Positive effects on smoking abstinence were found of the Internet-based multiple computer-tailored smoking cessation programme for three subgroups of smokers recruited via general practices: smokers with low levels of addiction, smokers who made many previous quit attempts and older smokers (chapter 5). The results from a study we conducted within a general population sample of smokers supported and extended these positive effects, as positive intervention effects on smoking abstinence rates were identified for the entire study population (chapter 6 ). The results from the economic evaluation study even further extended our recommendation as the Internet-based multiple computer-tailored smoking cessation programme was found to most probably be the most cost-effective treatment, assuming a willingness to pay of $€ 5,100$ per abstinent smoker (chapter 7). No intervention effects on smoking abstinence were identified when one tailored feedback letter was replaced by a tailored counselling session by a practice nurse; nor did this intervention appear to be cost-effective.

\section{Implications for theory and future research}

While positive effects of the Internet-based multiple computer-tailored smoking cessation programme on smoking abstinence were identified in both studies that evaluated the effectiveness of PAS, these effects did only persevere over relatively short periods of time. This was unexpected, as a single tailored feedback message was yet able to increase smoking abstinence rates (Te Poel et al., 2009) and multiple tailoring has been found to further increase effectiveness (Borland et al., 2004, Dijkstra et al., 1998c). However, an important difference exists between the studies presented in this dissertation and the study testing the single tailored intervention developed by te Poel et al. (2009). In the latter study, in addition to feedback tailored to cognitive variables, intention and behaviour, respondents in the intervention group received computer-tailored feedback on action planning, including a personalized overview of the action plans (e.g. planning a quit date) the participant intended to implement (Te Poel et al., 2009). The control group received information that was similar in content, but non-tailored and did not include a personalized action plan overview (Te Poel et al., 2009). In contrast, all smokers participating in the studies about the effectiveness of PAS were encouraged to set a quit date within four weeks from baseline, also when part of the control group. As emphasized by te Poel et al. (2009), planning a quit date is a form of action planning that has a positive influence on cessation behaviour (Balmford et al., 2010). While in both effectiveness studies presented in this dissertation smoking abstinence rates were higher in the intervention than in the control group, the fact that respondents in the 
control group were encouraged to set a quit date as well might explain why these differences on the longer term were only modest and non-significant. To determine the effectiveness of each intervention component, including setting a quit date, separately, future studies should aim at disentangling the working mechanisms of computer-tailored interventions such as PAS and the one developed by te Poel et al. (2009).

A second potential explanation for the lack of long-term effects may come from our use of motivation as an inclusion criterion. In accordance with inclusion criteria used in other studies testing the effectiveness of online smoking cessation programmes (Hoving et al., 2010, Te Poel et al., 2009), smokers were eligible to participate when motivated to quit within one month (preparers) or within six months (contemplators). However, regardless of their initial motivation to quit, all respondents were invited to set a quit date within four weeks from baseline. Based on positive results from previous studies (Dijkstra et al., 1998a, Te Poel et al., 2009), it was hypothesized that completion of the questions on behaviour, intention and cognitions about smoking and non-smoking and, in the intervention groups, receipt of feedback tailored to respondents' answers to these questions, would result in an increased motivation to quit and a willingness to set a quit date within this time frame. In line with this reasoning, we observed high response rates to this question (88-91\%). However, while no differences in attrition rates could be detected between preparers and contemplators, significant differences were observed with regard to reported quit attempts and smoking abstinence. That is, contemplators were significantly less likely to undertake a quit attempt before, on or after their set quit date and were less likely to report smoking abstinence during the intervention period than preparers. This suggests that despite the fact that contemplators set a quit date when encouraged to do so, these smokers were not sufficiently motivated to actually make an attempt to quit and/or to remain abstinent from smoking when they made a quit attempt. As approximately one third of participating smokers were categorized as contemplators, future studies should investigate whether these smokers benefit from increased efforts to enhance participants' motivation to quit and to move them to preparation before encouraging them to set a quit date within the near future.

A third possible explanation might be that the Internet-based multiple computer-tailored smoking cessation programme was not sufficiently adapted to the long-term wishes of recent ex-smokers to prevent relapse to smoking. Previous research has shown that ex-smokers' relapse to smoking can be predicted by low levels of self-efficacy (Gwaltney et al., 2009), negative outcome expectancies of nonsmoking (Dijkstra and Borland, 2003, Gwaltney et al., 2005), a lack of social support (Lawhon et al., 2009), higher levels of nicotine dependence (Hyland et al., 2006, Zhou et al., 2009) and craving (Killen and Fortmann, 1997), as well as by negative affect and stress (Kassel et al., 2003). So far, behavioural relapse prevention interventions have mainly focussed on the identification of and coping with difficult situations (Hajek et al., 2009). Similarly, PAS included a coping planning component 
focused on potential high-risk situations as this showed positive effects on relapse prevention in previous studies (e.g. Hoving et al., 2006b, van Osch et al., 2008). The systematic review conducted by Hajek et al. (2009), however, shows that relapse prevention interventions that focus on identifying and resolving tempting situations have only limited effects on behaviour. This suggest that to improve the Internet-based multiple computer-tailored smoking cessation programme, relapse prevention strategies should be incorporated that not only focus on the identification of and coping with high-risk situations, but also target other predictors of smoking relapse, such as low levels of self-efficacy and negative affect.

Another recommendation for future research comes from the results of the economic evaluation study described in this dissertation. While a willingness to pay of $€ 18,000$ per QALY is an accepted Dutch cut-off point when it concerns preventive interventions such as PAS (Casparie et al., 1998), no such cut-off point currently exists with regard to abstinence rates. When compared with care as usual, in the Internet-based multiple computer-tailored programme $€ 5,100$ had to be paid for each additional abstinent respondent. This is slightly more than what was found in a previous study (Salize et al., 2009). This study showed that compared with treatment as usual, €92 per patient had to be invested to gain one additional quitter in an intervention consisting of training for general practitioners and cost-free nicotine replacement medication and/or bupropion hydrochloride for patients (Salize et al., 2009). However, the study by Salize et al. (2009) investigated the costeffectiveness of a smoking cessation intervention very different from the behavioural intervention studied in this dissertation. Furthermore, it only included costs directly related to the intervention received, whereas we conducted our economic evaluation study from a broader societal perspective and included intervention costs, health care costs as well as patient costs. Results are thus not very comparable. Besides, it is currently unknown what society would be willing to pay per abstinent smoker, which hinders the interpretation of the incremental costs per abstinent participant. Previously, attempts have been made to identify the willingness to pay for health improvements of physical activity on prescription (Rome et al., 2010), for obesity treatment (Narbro and Sjostrom, 2000) and for a cardiovascular prevention programme (Jacobs et al., 2011a). Yet, no such studies have been conducted to identify the willingness to pay for an abstinent participant or for a behavioural smoking cessation intervention such as PAS. Therefore, future research should aim to identify these thresholds, for society in general and for the Dutch society in particular.

A last finding that holds implications for future research is that no effects on smoking abstinence were identified of the combination of the Internet-based multiple computer-tailored smoking cessation programme and tailored counselling by a practice nurse. Based on results from previous studies (Curry et al., 1995, Sutton and Gilbert, 2007), we hypothesized that the replacement of one tailored feedback letter by a single, face-to-face counselling session with a practice nurse and the addition of a telephone call six months after baseline would significantly increase 
the effect of the Internet-based programme. Based on the results presented, it appeared that this was not the case. Even though the content of the counselling sessions and the content of the computer-tailored feedback letters were developed to be as similar as possible, a potential explanation for this finding might be that control over the delivery of this content could not be exerted to a similar extent in case of both delivery modes. Using computer-tailoring technology, to a great extent control can be exerted over the content of the information delivered (de Vries and Brug, 1999, Kreuter et al., 1999), ensuring discussion of pre-defined issues related to smoking and non-smoking. On the contrary, the information delivered via counselling sessions may have been subject to practice nurses' usual smoking cessation counselling routine, their knowledge and remembrance of the PAS counselling protocol during counselling sessions as well as to time constraints within the general practice (Hall and Marteau, 2007, Nagelhout, 2007). While we aimed to take account of this potential barrier by asking practice nurses to fill out a checklist of each counselling session, functioning both as a registration system and reminder, practice nurses' compliance with filling out these checklists was limited. As a consequence, it cannot be guaranteed that each counselling session covered all smoking and non-smoking related issues included in the PAS counselling protocol. The idea that adherence to the counselling protocol may have been imperfect is supported by earlier research indicating that, although evidence-based, the Minimal Intervention Strategy for general practices (H-MIS) was not optimally implemented by general practice staff (Kwaliteitsinstituut voor de Gezondheidszorg CBO, 2004). That is, less than $30 \%$ of Dutch general practitioners (GPs) reported to use this guideline and it is unknown to what extent these GPs adhered to this guideline (Kwaliteitsinstituut voor de Gezondheidszorg CBO, 2004). In addition, while an understanding and supportive tone was used to develop computer-tailored feedback messages, no influence could be exerted on the way in which practice nurses communicated about smoking and smoking cessation with their patients. Previous research suggested that a relatively controlling counselling style might potentially be counter-productive when promoting smoking cessation (Williams et al., 2002, Williams et al., 2006a). This lack of control over the content and style of the counselling sessions delivered by participating practice nurses may have resulted in less than optimal results of the combined intervention. Therefore, future research might need to focus on investigating the level of adherence to counselling protocols such as the one described in this dissertation, for instance by audio- or video-taping counselling sessions or by using clinical vignettes (Rutten et al., 2009). Moreover, to be able to increase adherence rates factors would need to be identified that facilitate, e.g. intervention cards (Segaar et al., 2007), or hinder, e.g. time constraints (Hall and Marteau, 2007, Nagelhout, 2007) adherence, as well as strategies to increase or decrease these factors, respectively. 


\section{Implications for practice}

Promising results were found regarding both the effectiveness and costeffectiveness of the Internet-based multiple computer-tailored smoking cessation programme. Before deciding to implement this intervention on a large scale, however, it should be improved.

First, the Internet-based multiple computer-tailored smoking cessation programme should be improved by incorporating relapse prevention strategies that go beyond the identification of and coping with potentially difficult situations. The current programme was not able to tailor feedback to daily fluctuations in predictors of lapses to smoking, like self-efficacy and negative affect (Gwaltney et al., 2005, Shiffman et al., 2007), and to provide feedback when smokers might have needed it most. This is due to the fact that within the Internet-based multiple computer-tailored smoking cessation programme, respondents received feedback only at fixed points in time. A strategy that could monitor fluctuations in predictors of lapses and relapse within an Internet-based computer-tailoring programme such as PAS is ecological momentary assessment (Gwaltney et al., 2005, Shiffman et al., 2007). Making use of mobile devices such as smart phones, the integration of this strategy would create the possibility to collect real-time data from recent exsmokers. Closely monitoring fluctuations in the predictors of lapses and relapse would subsequently enable us to more instantly provide feedback tailored to, for instance, ex-smokers' levels of self-efficacy and negative affect. A first study towards the effectiveness of integrating ecological momentary assessment within an Internet-based multiple computer-tailored smoking cessation programme is currently being conducted within our research group (Elfeddali et al., 2012b).

Secondly, many participants discontinued the Internet-based multiple computer-tailored smoking cessation programme, a phenomenon also seen in other web-based interventions (Blankers et al., 2010, Eysenbach, 2005, McKay et al., 2008, Shahab and McEwen, 2009, Webb, 2009, West et al., 2005). At the same time, previous research identified a significant dose-response relationship between the number of feedback moments and smoking abstinence (Krebs et al., 2010, Strecher et al., 2008). In addition, a recent systematic review of Internet-based therapy for the treatment of addictions (Gainsbury and Blaszczynski, 2011), such as the smoking of tobacco, found that respondents who more actively used the intervention materials, such as websites and e-mail, were more likely to have successful treatment outcomes (Gainsbury and Blaszczynski, 2011). It is therefore of utmost important that smokers complete the intervention they participate in. Previously, several suggestions have been made to increase retention rates among smokers, such as ensuring high levels of motivation to quit, providing prompts or reminders, preventing self-control depletion, for example by having respondents form implementation intentions (Webb, 2009), and providing incentives of at least $€ 10$ (Khadjesari et al., 2011). A recent review showed that interventions that combined several of these strategies were most effective in optimizing exposure to web-based interven- 
tions (Crutzen et al., 2011). Although within PAS all strategies mentioned were taken into account, attrition rates remained high. Evidently, more research is needed to identify strategies that will prevent smokers from dropping out of this intervention and of similar interventions. A first step might be to conduct qualitative research among respondents lost to follow-up; this might further illuminate the main reasons for these respondents to discontinue a web-based intervention. Based on the results from such research, novel strategies to prevent attrition need to be developed, tested and, when effective, incorporated within web-based programmes.

Furthermore, as contemplators were less likely to undertake an attempt to quit smoking than preparers, the Internet-based multiple computer-tailored smoking cessation programme could be improved by making a greater effort to increase participating smokers' motivation to quit. This might be achieved by incorporating strategies derived from Self-Determination Theory (SDT) (Ryan and Deci, 2000) and Motivational Interviewing (MI) (Miller and Rollnick, 1991, Miller and Rollnick, 2009) in web-based smoking cessation interventions; a further elaboration on these ideas is described in the section of this general discussion that elaborates on the construct of motivation.

\section{DISSEMINATION}

\section{Main findings}

Both a positive attitude and a positive social environment were positively associated with the intention to adopt and to implement PAS (chapters 3 and 8). Practice nurses' and nurse practitioners' satisfaction with their current smoking cessation activities was negatively correlated with the intention to adopt, whereas involvement in the trail defined in terms of recruitment success was positively associated with practice nurses' intention to implement. Furthermore, while recruitment via general practices yielded much fewer respondents than recruitment via the mass media, respondents recruited via general practices were more likely to quit smoking: they were significantly less addicted to nicotine, more motivated to quit smoking as well as to maintain non-smoking and they were more likely to complete the intervention (chapter 4).

\section{Implications for theory and future research}

While previous implementation studies have tried to integrate Rogers' innovation characteristics within the socio-cognitive concept of attitude (Bolman et al., 2002a, Segaar et al., 2007), based on the results presented in this dissertation it can be suggested that attitude and innovation characteristics might need to be considered as two separate and independent constructs. This is in accordance with suggestions from previous implementation studies that attitude should be considered as a characteristic of the intervention's user (i.e. the health professional) and that Rogers' concepts should be regarded as characteristics of the intervention, as perceived by its user (Fleuren et al., 2004, Helmink et al., 2011). In other words, attitude might 
consist of general beliefs about an intervention, e.g. whether the intervention is effective or not, whereas Rogers' innovation characteristics' may represent more specific perceptions of the interventions' characteristics also taking into account current practice, e.g. whether it the intervention is perceived as more effective than currently used programmes. So far, the idea that attitude and innovation characteristics are two separate and independent constructs has received little attention. Future longitudinal research should further investigate this idea; ultimately, this might result in more specific targets for developing intervention strategies aimed at increasing implementation rates.

Furthermore, besides focussing on well-investigated cognitive factors such as attitude and social influence, studies investigating the dissemination of innovations might need to take two additional factors into account: the health professional's satisfaction with current practice and the health professional's involvement during a trial period. Both factors could be considered behavioural factors, a category within the predisposing factors included in the I-Change Model (ICM) (De Vries et al., 2003). As the other predisposing factors, behavioural factors are regarded as external factors thatcan have a direct influence on behaviour, but are also assumed to influence a person's behavioural intention indirectly through awareness, attitudes, social influence perceptions and self-efficacy expectations (de Vries and Mudde, 1998). While involvement appeared to have a direct influence on the intention to implement, the association between satisfaction and the intention to adopt was suggested to be mediated by attitude. This could be explained by principles of persuasion; incomplete satisfaction or dissatisfaction with one's own behaviour can be considered as one of the intrapersonal conditions which people try to solve or remove in order to achieve cognitive consonance (Rossiter and Percy, 1987). As both satisfaction with current practice and the health professional's involvement during a trial period appeared to play a significant role in the dissemination process, it is recommended to include these factors in future studies towards the dissemination of interventions in primary care. Yet, further longitudinal research is needed to investigate whether these factors predict the intention to adopt and implement, respectively, in a direct or indirect way. Preferably such research should be conducted using relatively advanced statistical techniques able to analyze the importance and interrelationship of multiple potential predictors, such as Structural Equation Modelling (SEM) analyses (Gerbing and Anderson, 1988).

Two of the three studies regarding the dissemination of PAS (chapters 3 and 8) had a cross-sectional design; there was only one point in time at which data was gathered from participating practice nurses (Bouter et al., 2005). This implies that no conclusions can be drawn about the causality of the associations found. Furthermore, both studies used the intention to adopt/implement as the main outcome measure. Although it would have been more desirable to study the determinants of actual adoption and implementation, at the moment that these studies were conducted PAS was not yet nationally available and it was thus impossible to assess actual adoption and implementation rates. Many theoretical frameworks, e.g. the 
Theory of Planned Behaviour (Ajzen, 1985) and the ICM (De Vries et al., 2003), have incorporated intention as the most proximal predictor of behaviour. In addition, a recent systematic review showed intention to be an important predictor of health professionals' clinical behaviour, explaining a similar proportion of the variance in behaviour found in studies among non-health professionals (Eccles et al., 2006). While intention can thus be considered a reliable precursor of practice nurses' actual adoption and implementation, the main recommendation for theory and future research is to conduct studies with a longitudinal character and with actual adoption and implementation behaviour as outcome measures.

\section{Implications for practice}

A positive attitude appeared to be associated both with increased adoption and increased implementation rates of PAS. In addition, Rogers' innovation characteristics were independently, positively associated with practice nurses' intention to implement PAS. This is in line with previous studies (Chavannes et al., 2007, Coleman et al., 2001, Hall and Marteau, 2007, Hall et al., 2005) and emphasizes the importance of creating a positive attitude towards the intervention among health professionals and to convince them of the intervention's beneficial characteristics. In the studies described in this dissertation practice nurses were provided with information about PAS via the PAS research team. It has, however, been suggested that the source of the message is an important predictor of attitude (De Vries et al., 2003, McGuire, 1985) and that the use of champions or key players might facilitate the dissemination of preventive interventions (Rogers, 2002). To increase a positive attitude towards adoption and implementation, it might therefore be better to disseminate information about a new intervention for practice nurses via key players among practice nurses, instead of via researchers they barely know or can identify with. In the Netherlands, key players might be sought, for instance, amongst active members of the V\&VN Dutch Nurses' Association or the Dutch Association for Practice Nurses (NVvPO).

Secondly, a positive social environment appeared to be associated with both the intention to adopt and the intention to implement PAS, which is in accordance with earlier research findings (Coleman et al., 2001, Hall and Marteau, 2007, Kaner et al., 2007, Nagelhout, 2007, Sinclair et al., 2008, Hoving et al., 2006a, Hoving et al., $2007 \mathrm{~b}$ ). While the social norm among colleagues appeared to be most important in the adoption process, perceived patient support was suggested to be especially important in explaining the intention to implementation the intervention. To increase dissemination rates, it is thus of foremost importance to create a positive social norm among colleagues. This might again be achieved by the identification of key players among practice nurses, as the diffusion of innovations is suggested to be a social process of people talking about the intervention, giving it meaning for themselves, and then adopting (Rogers, 2002). After the initial adoption of the intervention, perceived support from patients should be increased. As most participating practice nurses did not experience much support from their patients, this 
definitely appears to be an area with room for improvement. One strategy to further stimulate implementation might be to more intensively involve patient organizations in the development of the intervention and to explicitly show their involvement to practice nurses (e.g. in promotion materials) (Rogers, 2002).

Furthermore, based on the results presented the choice for a particular recruitment strategy can be expected to influence an intervention's potential public health impact. That is, recruitment strategy not only appeared to have an influence on the number and type of smokers who agree to participate in an Internet-based smoking cessation programme, but, consequently, also on retention rates and smoking cessation behaviour. This emphasizes that the choice for a particular recruitment strategy should first of all be informed by the target population the intervention has been developed for. In line with previous research (Etter and Perneger, 2001a), it could be recommended that when the target group consists of smokers with a low socio-economic status in particular, the general practice may be the most suitable gateway for recruitment. When the target group does, however, consist of smokers including but not limited to lower socio-economic status smokers, mass media recruitment might be a better strategy as a larger absolute number of smokers might be reached. The same counts for smokers with chronic diseases such as cardiovascular and respiratory diseases; if these are the main target group recruitment via general practices might be the best option.

\section{MOTIVATION}

\section{Main findings}

When disentangling the roles played by the different types of motivation defined by PRIME theory (i.e. desire, duty and intention) (West and Hardy, 2006), it appeared that duty (i.e. the belief that one ought to quit) was the most commonly reported motive for quitting smoking. Nevertheless, only a desire and intention to quit smoking were significant independent predictors of quit attempts (chapter 9). Furthermore, when investigating whether subtypes exist among smokers in contemplation, it was revealed that within the contemplation stage of change three clusters of smokers could be identified: Early, Progressing and Disengaged Contemplators (chapter 10). Interestingly, no cluster was identified representing the Classic Contemplator.

\section{Implications for theory and future research}

The results presented were not all in line with expectations derived from the theoretical frameworks used. As a consequence, these findings suggest relevant directions for future theoretical research.

First, the finding that duty did not predict quit attempts was in line with predictions from PRIME theory (West and Hardy, 2006). However, duty unexpectedly mitigated the effect of desire and intention. This is consistent with previous research suggesting that a sense of external pressure might potentially be counter- 
productive when promoting smoking cessation (Williams et al., 2002, Williams et al., 2006a) and might suggest to investigate whether PRIME theory should be expanded with a negative association between duty and behaviour. Besides, the finding that intention appeared to predict quit attempts independently from desire was not expected based on PRIME theory either. As there is an abundance of literature showing that intention is the most proximal predictor of behaviour change in general (Armitage and Conner, 2001, Godin and Kok, 1996) and of smoking cessation behaviour in particular (Armitage and Conner, 2001, Hyland et al., 2006, Norman et al., 1999), PRIME theory's assumption that intention only has an influence on behaviour through desire may need to be reconsidered. These two possible amendments to PRIME theory should be tested in future studies, preferably using relatively more advanced statistical techniques such as SEM analyses, which are able to model the relationships between multiple independent and dependent constructs simultaneously (Gerbing and Anderson, 1988).

Secondly, as the study presented in chapter 9 was one of the first explicitly testing predictions from PRIME theory (West and Hardy, 2006), more research needs to be conducted to investigate the psychometric properties of the measurement scales of the three constructs, i.e. desire, duty and intention, proposed by this theory. First, however, these measures need to be expanded to include a minimum of three items per theoretical construct (Field, 2005). Measurement items additional to the items used in our own research could be developed using input from experts and potential end-users, i.e. smokers, as well as existing measurement instruments. It could be argued, for instance, that a desire to quit resembles intrinsic or autonomous motivation, while a sense of duty may be similar to a extrinsic or controlled motivation (Ryan and Deci, 2000, Curry et al., 1990). Similarly, although less prominent than in PRIME theory and SDT, several socio-cognitive theories have suggested a distinction between intrinsic and extrinsic perceived advantages and disadvantages of a behaviour (De Vries et al., 2003, Bandura, 1986). Furthermore, the definition of intention as described in PRIME theory can be considered similar to the definition included in socio-cognitive theories like the Theory of Planned Behaviour (TPB) (Ajzen, 1985) and ICM (De Vries et al., 2003). Psychometric properties of the measurement scales developed should subsequently be evaluated according to previously defined quality criteria (e.g. Terwee et al., 2007, Scientific Advisory Committee of the Medical Outcomes Trust, 2002).

Thirdly, based on the results presented the idea of theoretical integration can be put forward. Within socio-cognitive models, such as the TPB (Ajzen, 1985) and the ICM (De Vries et al., 2003), intention is included as the most proximal predictor of behaviour. In turn, intention is assumed to be predicted by cognitive beliefs and expectations like attitude, social influence and self-efficacy (Ajzen, 1985, De Vries et al., 2003). Based on the results presented, though, it could be suggested that albeit that intention might be an independent predictor of undertaking an attempt to quit smoking, so might be a desire to quit, whereas duty may have a negative effect on quit attempts. While not many studies to date have shown this pattern of results 
using PRIME theory of motivation (West and Hardy, 2006) as a theoretical framework, these findings are supported by the results from research based on SDT (Ryan and Deci, 2000). That is, while a sense of autonomy has been found to be important when quitting smoking, perceived external pressure has been found to potentially be counter-productive (Williams et al., 2002, Williams et al., 2006a). Within SDT the construct of intention is not explicitly incorporated. Yet, this theory might offer explanations for the origins of the cognitive beliefs and expectations assumed to predict intention within socio-cognitive theories (Deci and Ryan, 1988, Vallerand, 1997). Support for this suggestion has yet been found with regard to physical activity and dieting behaviour, a meta-analysis by Hagger and Chatzisarantis (2009). It is, however, also conceivable that a relatively intrinsic (rather than extrinsic) motivation to quit smoking is a direct predictor of an intention to quit or even that motivation functions on a similar level as intention, i.e. is a direct predictor of behaviour. Future research is required to determine whether the construct of motivation, thereby distinguishing between intrinsic and extrinsic types of motivation, adds predictive value with regard to smoking cessation behaviour when incorporated within existing socio-cognitive models such as the TPB and ICM and to investigate where this construct should be placed within these theories. While attempts at such theoretical integration have been made within the field of physical activity and dietary behaviour (e.g. Hagger and Chatzisarantis, 2009, Jacobs et al., 2011b), within the field of smoking cessation research there is a shortage of such research.

Fourthly, unlike previous studies that focused on clusters of smokers within the contemplation stage of change (Anatchkova et al., 2005, Norman et al., 2000, Velicer et al., 1995), we were unable to identify a cluster representing the so-called Classic Contemplator, characterized as having above average scores on both the pros and cons of smoking and high scores on the perceived temptations to smoke (Anatchkova et al., 2005, Norman et al., 2000, Velicer et al., 1995). Nonetheless, the cluster solution presented here is expected to be more accurate than the cluster solutions found in earlier research, as clustering variables were measured in a similar manner as the target behaviour (i.e. related to quitting smoking instead of smoking), which is in accordance with Ajzen's compatibility principle (Ajzen, 1988). This suggests that the Classic contemplator might not, or no longer, exist. As the three clusters of smokers we did identify are comparable to those found in previous studies (Anatchkova et al., 2005, Norman et al., 2000, Velicer et al., 1995), however, a substage theory consisting of three stages within the contemplation stage of change, might deserve further investigation. To evaluate the validity of a stage theory, Weinstein (1998) has suggested four types of research that need to be conducted: 1) cross-sectional comparisons of people in different stages; 2) examination of stage sequences; 3) longitudinal prediction of stage transitions; and 4) experimental studies of matched and mismatched interventions. While several attempts have been made to validate the original stages of change by conducting at least the first three types of research (e.g. Dijkstra et al., 2006, Hoving et al., 2006c), no such ex- 
tensive attempts have been made with regard to substage theories. Together with prior studies focusing on subtypes within contemplation (Anatchkova et al., 2005, Norman et al., 2000, Velicer et al., 1995), the study presented in chapter 3 of this dissertation covers the first type of research. However, while the cross-sectional comparison of the substages lends support for the proposed substage theory, this type of research has been argued to only provide a weak test of stage ideas (Weinstein et al., 1998). Before being able to draw definite conclusions about the validity of the proposed substage theory, it is required that the other three types of research are conducted as well (Weinstein et al., 1998). When ultimately proven valid, a substage theory like the one proposed might provide opportunities to further tailor smoking cessation interventions to the needs of groups of individuals with similar characteristics. As it has previously been suggested, though, that a continuous measure of intention is perhaps a better predictor of behavioural change than the stages of change (de Vet et al., 2007), as an additional check of the proposed substage theory future studies should investigate the relative predictive power of the substages when compared with continuous measures of motivation and readiness to change.

\section{Implications for practice}

Although the results presented should be interpreted with some caution, based on these results improving alterations to the Internet-based multiple computertailored smoking cessation programme described in this dissertation can be suggested. As described in the evaluation section of this general discussion, contemplators were less likely to undertake an attempt to quit smoking than preparers. Within the present version of the intervention, which is based on the ICM (De Vries et al., 2003), we have tried to increase smokers' intention to quit by providing tailored feedback on cognitive factors such as respondents' attitude towards smoking, perceived social influence and their levels of self-efficacy to quit. While the programme provided smokers with feedback regarding perceived pros and cons of smoking cessation that could be intrinsic or extrinsic to the person, no emphasis was placed on intrinsic reasons to quit smoking, such as quitting for the sake of one's health or because it makes you feel better, over extrinsic reasons to quit, such as perceived social pressure. Yet, based on the results presented it could be argued that different types of motivation may have a different influence on smoking cessation behaviour. That is, although a sense of duty was the most commonly reported motive for quitting smoking, it was not a predictor of smokers' attempts to quit; only a desire or intrinsic motivation and an intention to quit smoking significantly predicted the occurrence of quit attempts. These findings suggest that, in addition to a continued focus on increasing smokers' intention to quit smoking in the near future, the Internet-based multiple computer-tailored smoking cessation programme may be further improved by an increased focus on enhancing smokers' intrinsic motivation to quit. This idea is supported by findings from studies that aimed to integrate socio-cognitive theory with constructs from SDT; these studies 
showed that an increase in autonomous motivation yielded more positive attitudes and higher levels of self-efficacy (Vallerand, 1997, Hagger and Chatzisarantis, 2008). Since these are two key determinants of the intention to change within socio-cognitive models of behavioural change, an increased autonomous motivation could be assumed to increase the intention to change too.

Previously, a factor that has been found to facilitate the internalization of autonomous motivation, as well as to increase perceived competence and smoking cessation rates, is autonomy support (Williams et al., 2002, Williams et al., 2006b). Practical strategies to increase autonomous motivation can be derived from SDT, but also from the more practice-oriented counselling approach of MI (Miller and Rollnick, 1991, Miller and Rollnick, 2009). Examples of such autonomy supportive strategies are: elicit the patient's perspectives, explore patient's values and how these relate to smoking (cessation) and provide effective options for change while acknowledging the option of not changing (Williams et al., 2011, Markland et al., 2005). Although health behaviour change interventions are increasingly delivered online (Lustria et al., 2009, Shahab and McEwen, 2009), so far MI has been used extensively as a basis for telephone or face-to-face counselling (Heckman et al., 2010), but only scarcely to guide Internet-based interventions. Recently, MI been used successfully to guide weight loss counselling sessions in chat-rooms (Webber et al., 2008) and counselling via e-mail to improve fruit and vegetable consumption (Alexander et al., 2010). In the Netherlands, similar research is currently being conducted with regard to physical activity behaviour. Despite promising results, no smoking cessation interventions have been developed and tested that explicitly incorporated SDT- or MI-based strategies. Though, within PAS some of these principles were to a certain extent yet taken into account implicitly. That is, smokers' perspectives were elicited by asking respondents to answer closed-ended questions about the perceived pros and cons of quitting smoking. To further improve this component respondents could be provided with room to describe pros and cons of quitting they might perceive in addition to those pre-defined within the closedended questions, as well as to describe perceived pros and cost of smoking, their current behaviour. Furthermore, smokers' values could potentially be taken into account more explicitly. Aspirations for physical health in general have been shown to facilitate tobacco abstinence (Niemiec et al., 2009). Within the ICM, such life aspirations could be considered as one of the psychological factors which are part of the predisposing factors included in this model (De Vries et al., 2003). To take account of this construct within the Internet-based multiple computer-tailored smoking cessation programme, smokers' life aspirations could be assessed, e.g. using closedended questions, while respondents could describe how these aspirations relate to smoking (cessation) behaviour in open-ended questions. Even though it might be difficult for a computer programme to provide tailored feedback based on answers to open-ended questions, this process might create awareness of potential discrepancies between general life aspirations and specific smoking-related behaviour. 
Another practical implication of the results presented in this dissertation concerns the traditional interventions targeting smokers in contemplation, which typically attempt to solve the cognitive conflict contemplators are said to experience by aiming to decrease the perceived cons of quitting (Dijkstra et al., 2006). As in our sample of smokers contemplating to quit no smokers were in such a cognitive conflict about their smoking behaviour, it could be argued that these interventions might not always be appropriate. Rather, it can be argued that smoking cessation interventions should pay more attention to the Disengaged Contemplator, as within our study almost half of the respondents were Disengaged. In our and previous studies (Anatchkova et al., 2005, Everitt, 1980, Norman et al., 2000, Velicer et al., 1995), the Disengaged Contemplator has been suggested to experience few advantages and disadvantages of quitting smoking, while reporting high levels of selfefficacy. While the Disengaged smoker might thus be able to quit, this subtype appears not to be interested in quitting smoking. Perhaps these smokers would benefit from interventions usually focused on precontemplators, as these aim at increasing the perceived pros of quitting (Dijkstra et al., 2006). As they already perceive few cons of quitting and a high self-efficacy to quit, they might then directly move to preparation or action. Yet, rather than to provide stage-matched information, assuming that smokers in the same stage benefit from targeting the same psychological constructs (Dijkstra et al., 2006, Prochaska et al., 2008), another possibility is to provide smokers with item-based feedback. Within item-based computer-tailoring smokers are provided with feedback tailored to cognitive variables, intention, goal and relapse prevention strategies and smoking behaviour, regardless of their stage of change. Based on the results presented it can be suggested that stage-matched interventions might not always be appropriate for smokers in contemplation and that an item-based approach, as also used within PAS, might be preferable.

\section{METHODOLOGICAL CONSIDERATIONS}

A randomized controlled trial was chosen as the most appropriate design for the two effectiveness studies described in this dissertation. This design is widely perceived as a 'golden standard' and as the strongest and most appropriate design to evaluate interventions for their effectiveness (Campbell et al., 2000, Flay, 1986, Flay et al., 2005). Despite the strong design used, the results presented in this dissertation should be interpreted in light of several methodological considerations which might have affected the quality of the studies conducted.

\section{Internal validity}

As the present dissertation aimed to investigate the (cost-)effectiveness and feasibility of the smoking cessation intervention PAS under real life circumstances, it compared the two versions of the PAS intervention with a control group that received care as usual, i.e. current smoking cessation help as delivered in the primary care setting. While this was expected to yield study results that would be of most 
pragmatic value, using a usual care control group had a major disadvantage with regard to our study's internal validity. That is, usual care for smoking cessation in Dutch general practices varies considerably (Chavannes et al., 2007, Partnership Stop met Roken, 2009). Variation in standard care provided to control groups has been suggested to substantially influence effect sizes of behaviour change interventions (de Bruin et al., 2009). As anecdotal evidence suggests that care as usual in our study was rather intensive, usually consisting of four to six smoking cessation consultations, it could be suggested that the effects of the intervention on smoking abstinence rates might have been underestimated. On the other hand, the variability in the usual care received by our control group might be inherent to the pragmatic approach used in effectiveness trials. The results presented might thus simply reflect real life in the general practice.

\section{External validity}

The external validity of the studies presented in this dissertation may be restricted for two reasons. First of all, the studies that were conducted to investigate the adoption and implementation of PAS were conducted amongst Dutch practice nurses and nurse practitioners and concerned an intervention developed for Dutch adult smokers and available in the Netherlands only. While the organization of primary care in the Netherlands is relatively similar to the organization of care in, for instance, England (Erler et al., 2011), it is considerably different from the organization of care in the Unites States (Erler et al., 2011, van Weel et al., 2012). When generalizing the findings from these studies to primary care settings in other countries than the Netherlands, some caution is thus warranted; one should be aware of similarities and differences that exist in the organization of health care between the different countries. Secondly, the two effectiveness trials described in this dissertation might have suffered from selection bias. This is supported by the findings described in chapter 4 of this dissertation: respondents recruited via mass media were significantly higher educated, more addicted to nicotine, less motivated to quit smoking and to maintain non-smoking, and suffered less from respiratory and cardiovascular diseases than those recruited via general practices. These differences in sample characteristics subsequently appeared to result in differences in retention rates and in differences in quitting behaviour between the two samples. Caution is therefore warranted when generalizing the findings from the two effectiveness trials to different populations of smokers.

\section{Measurement of smoking abstinence}

For the majority of the studies described in the present dissertation, the outcome measures used were prolonged abstinence from smoking, seven-day point prevalence abstinence and 24-hour point prevalence abstinence. While positive intervention effects could consistently be detected on seven-day and 24-hour point prevalence abstinence rates, no intervention effects could be identified with regard to 
prolonged abstinence assessed after six (chapter 5) or after twelve (chapter 6) months. A potential explanation for this finding is that respondents might have experienced a lapse (or lapses) to smoking during the intervention period. Although lapses have been shown to strongly predict relapse, not all smokers who lapse ultimately relapse (Ockene et al., 2000, Shiffman, 2005). Some respondents may thus not have been prolonged abstinent, as they lapsed during the follow-up period, but have been abstinent for a long period of time. The definition of prolonged abstinence might have been too strict to categorize these smokers as abstinent. The Russell standard (West et al., 2005) takes account of this potential misclassification by allowing for a grace period until respondents' set quit date, a recommendation we adhered to within our studies. In addition, however, the Russell standard allows for a maximum of five cigarettes to be smoked throughout the entire follow-up period when assessing prolonged abstinence (West et al., 2005), a recommendation we did not comply with. The Russell standard acknowledges that this cut-off point for the number of slips allowed is arbitrary (West et al., 2005). Future studies would therefore benefit from explicitly investigating the occurrence of lapses during the follow-up period, from assessing the exact number of cigarettes smoked during these lapses and from investigating novel measures of smoking abstinence.

\section{PRACTICAL CONSIDERATIONS}

Several practical issues should be considered when interpreting the results from the studies described in this dissertation. In addition, it might be valuable to consider these practice issues when preparing or conducting comparable research.

\section{Recruitment of smokers}

As has been shown previously, the recruitment of smokers to smoking cessation trials is challenging (Gilbert et al., 2012). Especially within the study described in chapter 6 of the present dissertation, where recruitment of smoking patients was the responsibility of participating practice nurses, recruitment certainly appeared to be difficult. Although we successfully included even more than the 80 practice nurses required according to our power calculation, their subsequent recruitment of 15 smoking patients appeared to be rather hard. To help them to recruit smokers for participation in the trial, practice nurses were provided with several recruitment materials (i.e. recruitment letters, website texts, desk displays, business cards, posters) and they also reported to use these materials. Nevertheless, recruitment did not yield the 1200 smokers needed according to our power calculation. Practice nurses were encouraged to discuss their participation in the project with the general practitioner they were working for, as well as to ask the general practitioner for his or her approval to participate. Yet, practice nurses themselves were responsible for the recruitment of smoking patients. The positive correlation found between perceived social support from colleagues and recruitment success suggests that higher levels of (explicit) support from, or a more active involvement in the 
recruitment process of, the general practitioner or practice assistant might be a strategy to yield increased recruitment rates. Furthermore, a combined approach, recruiting smokers through general practices and mass media simultaneously, would be a strategy to increase recruitment rates. For such a combined approach it would, however, be required that PAS is implemented nationwide and that all approximately 5580 practice nurses in the Netherlands (Hingstman and Kenens, 2007, Hingstman and Kenens, 2010) are able to refer their smoking patients to this intervention. Based on the results presented in this dissertation, however, it can be concluded that before nationwide implementation of the Internet-based multiple computer-tailored smoking cessation programme, it should first be further improved.

\section{Drop-out of smokers}

An important limitation of both effectiveness trials described in this dissertation (chapters 5 and 6), as well as of the study conducted to investigate the role of different types of motivation in predicting attempts to quit smoking (chapter 9), is that they suffered from relatively high rates of attrition. While the attrition rates found were not extraordinary when compared to other studies of Internet-based interventions (McKay et al., 2008, Shahab and McEwen, 2009, Wangberg et al., 2008), they have some implications for the interpretation of the results presented.

First, in the study towards the effectiveness of PAS in a general practice setting, high rates of attrition resulted in the study being relatively underpowered to conduct some of the (subgroup) analyses described. This was demonstrated by several wide confidence intervals found in the regression analyses and the inability to detect statistically significant intervention effects within subgroups based on age and the number of previous quit attempts. As a sensitivity analysis whereby missing values were replaced using a negative scenario (i.e. drop-outs were regarded as smokers) was only able to partly replicate the intervention effects found, the results from this study should be interpreted with caution. Nevertheless, it is worth mentioning that both the results from the effectiveness trial conducted in a general population sample of smokers and the results from the economic evaluation study were in line with the results presented; they also suggested the Internet-based programme to be the most (cost-)effective.

Secondly, to prevent a lack of power, in the study towards the effectiveness of PAS among a general population sample of smokers missing values on the primary outcome measures were replaced using a negative scenario. Although this is a recommended analysis when having to deal with high rates of attrition (West et al., 2005), the replacement of more than $70 \%$ of the values on the primary outcome measures assuming this negative scenario might have increased the chances of making a type 2 error (Field, 2005, Eysenbach, 2005). This may have resulted in an underestimation of the intervention's effectiveness for those who continued to use it (Eysenbach, 2005). Nevertheless, the results from complete-case analyses were only slightly more positive. 
Both complete-case analysis and the replacement of missing values using a negative scenario have their limitations. It is therefore important that alternative strategies for dealing with missing data due to high attrition rates are identified and tested. A recent study comparing six different approaches to missing data concluded that multiple imputation might yield the most valid results (Blankers et al., 2010). Despite the fact that the replacement of missing values on outcome measures using a negative scenario is well-established and a still recommended strategy within smoking cessation research (West et al., 2005), the use of multiple imputation to replace missing values seems promising and warrants further investigation.

\section{Selective drop-out}

In both studies respondents who dropped out were relatively younger than those who remained in the study. This is in line with findings from previous research (Strecher et al., 2008, Wangberg et al., 2008). A potential explanation for this finding might be that younger people have not yet experienced any smoking-related health effects and are, compared to older people who are more often confronted with chronic diseases, less internally motivated to invest time in health behaviour change interventions (de Nooijer et al., 2005). This idea is supported by the data collected for this dissertation, showing significantly less cardiovascular diseases, COPD, diabetes and cancer among the relatively younger participants. Therefore, future research should focus on the identification of strategies able to prevent attrition, especially among these younger respondents. Furthermore, respondents who were experiencing more depressive symptoms were more likely to drop-out from the study described in chapter 6 of this dissertation. Previously, depressive symptoms were found to be positively associated with withdrawal symptoms (i.e. the more depressive symptoms, the more withdrawal symptoms) and negatively associated with smoking cessation outcomes (i.e. the more depressive symptoms, the less smoking cessation) (Leventhal et al., 2008). Nonetheless, future studies should aim to replicate our finding that depressive symptoms were associated with attrition. A last finding suggests that respondents who dropped out of the study presented in chapter 5 were relatively more addicted than those who remained in the study. This is in line with previous research (Strecher et al., 2008, Wangberg et al., 2008) and supports our idea that in the Internet-based tailoring programme not sufficient attention was paid to dealing with withdrawal symptoms.

\section{Cotinine validation}

When investigating the effectiveness of interventions aimed at smoking cessation, it has been suggested that biochemical validation is warranted to test whether selfreported behaviour is accurate (Patrick et al., 1994). In the effectiveness studies presented in this dissertation, practice nurses (general practice setting) and respondents themselves (general population setting) were responsible for conducting cotinine tests in case of self-reported abstinence from smoking twelve months after baseline. While in both studies self-reported abstinence was confirmed of almost all 
respondents who successfully underwent a cotinine test, response rates were low (48\% and 59\%, respectively). As a result, doubts may arise concerning the practicability of assigning the responsibility for conducting cotinine tests to practice nurses or respondents themselves.

An alternative approach to ours would be to have cotinine assessments be conducted by a research assistant, an approach that has been used in another smoking cessation study conducted within our research group (Elfeddali et al., 2012b). Within this study, a research assistant visited each respondent who reported to be abstinent at a convenient place (e.g. at home or at work) to collect saliva from the respondent using a swab stick. As within this approach the research team was able to exert control over cotinine assessment, this approach yielded almost $100 \%$ response rates. This approach is, however, relatively time-consuming and, as a consequence, costly.

Potentially the most practical alternative to improve the truthfulness of selfreports might be to use a 'bogus pipeline' procedure whereby respondents are led to believe that physiological measurements will assess their true smoking behaviour (Aguinis et al., 1993). As cotinine validation might not always be feasible, as indicated by the two effectiveness studies described, and previous studies have shown that self-reported smoking status is often a fairly accurate measure of actual smoking status (Patrick et al., 1994, Velicer et al., 1992), this might be a good alternative for actual biochemical validation.

\section{GENERAL CONCLUSIONS}

Based on the results from the studies presented in this dissertation, the following conclusions can be drawn with regard to the evaluation and dissemination of PAS and regarding the construct of motivation.

\section{Evaluation}

The Internet-based multiple computer-tailored smoking cessation programme was suggested to be effective in increasing short-term smoking abstinence rates among a general population sample of smokers and for subgroups of smokers, i.e. those with a low addiction level, those who were older and those who made many previous quit attempts, recruited in a general practice setting. The results from the economic evaluation study conducted extended our recommendation of the Internetbased programme to the entire study population, assuming a willingness to pay of $€ 5,100$ per abstinent smoker. While this might suggest nationwide implementation of the Internet-based multiple computer-tailored smoking cessation programme, PAS should first be further improved by incorporating more relapse prevention strategies such as the integration of ecological momentary assessment, by increasing its focus on enhancing intrinsic motivation and by integrating strategies aimed to prevention attrition. 


\section{Dissemination}

For the successful dissemination of PAS and similar interventions, it is especially important to create a positive attitude towards the intervention (e.g. by using champions to spread information about the intervention) and to create a positive social environment (e.g. by identifying key players among practice nurses). In addition, practice nurses should be offered help in the recruitment of their smoking patients, e.g. by more actively involving the general practitioner or practice assistant with in the recruitment process. Furthermore, the reach of PAS amongst the target population appeared to be influenced by the recruitment strategy used. While recruitment via general practices may attract smokers who are more likely to quit smoking, recruitment using a mass media approach might be able to reach a larger number of smokers and may ultimately lead to a larger absolute number of smokers quitting. As the choice for a particular recruitment strategy is consequently expected to influence the potential public health impact of the intervention, this choice should be conscious and based on the intervention's objectives.

\section{Motivation}

Testing predictions from PRIME theory of motivation revealed that duty was the most commonly reported motive for quitting smoking. Nevertheless, only a desire and intention to quit smoking were significant independent predictors of quit attempts. This suggests that interventions aimed at promoting smoking cessation behaviour need to go beyond creating the belief that smokers ought to quit smoking and make them want to quit and/or intend to quit smoking in the near future, e.g. by incorporating strategies derived from SDT and MI. Furthermore, as the contemplation stage of change might not, or no longer, be a genuine stage within the behavioural change process, traditional interventions for contemplators might no longer be appropriate. Smoking cessation interventions should specifically pay attention to Disengaged Contemplators, reporting few advantages and disadvantages of quitting smoking and high level of self-efficacy, as these smokers appear not to be interested in quitting and might consequently be the hardest to change. These smokers might benefit most interventions which have traditionally focused on precontemplators, but based on the results presented it could be argued that item-based instead of stage-based computer-tailoring interventions might be preferable. 


\section{REFERENCES}

ABRAMS, D. B., ORLEANS, C. T., NIAURA, R. S., GOLDSTEIN, M. G., PROCHASKA, J. O. \& VELICER, W. (1996) Integrating individual and public health perspectives for treatment of tobacco dependence under managed health care: a combined stepped-care and matching model. Annals of Behavioral Medicine, 18, 290-304.

AGUINIS, H., PIERCE, C. A. \& QUIGLEY, B. M. (1993) Conditions under which a bogus pipeline procedure enhances the validity of self-reported cigarette smoking: A meta-analytic review. Journal of Applied Social Psychology, 23, 352-373.

AHERN, D. K., KRESLAKE, J. M. \& PHALEN, J. M. (2006) What is eHealth (6): perspectives on the evolution of eHealth research. Journal of Medical Internet Research, 8, e4.

AJZEN, I. (1985) From intentions to actions: a theory of Planned Behaviour. IN KUHL, J. B., J. (Ed.) Action-control: From cognition to behavior. Heidelberg, Springer.

AJZEN, I. (1988) Attitudes, personality and behavior, Milton Keynes, Open University Press.

ALEXANDER, G. L., MCCLURE, J. B., CALVI, J. H., DIVINE, G. W., STOPPONI, M. A., ROLNICK, S. J., HEIMENDINGER, J., TOLSMA, D. D., RESNICOW, K., CAMPBELL, M. K., STRECHER, V. J. \& JOHNSON, C. C. (2010) A randomized clinical trial evaluating online interventions to improve fruit and vegetable consumption. American Journal of Public Health, 100, 319-26.

ALLENDER, S., BALAKRISHNAN, R., SCARBOROUGH, P., WEBSTER, P. \& RAYNER, M. (2009) The burden of smoking-related ill health in the UK. Tobacco Control, 18, 262-7.

ANATCHKOVA, M. D., VELICER, W. F. \& PROCHASKA, J. O. (2005) Replication of subtypes for smoking cessation within the contemplation stage of change. Addictive Behaviors, 30, 915927.

ANATCHKOVA, M. D., VELICER, W. F. \& PROCHASKA, J. O. (2006a) Replication of subtypes for smoking cessation within the precontemplation stage of change. Addictive Behaviors, 31, 1101-1115.

ANATCHKOVA, M. D., VELICER, W. F. \& PROCHASKA, J. O. (2006b) Replication of subtypes for smoking cessation within the Preparation stage of change. Addictive Behaviors, 31, 359-366.

ANNEMANS, L., NACKAERTS, K., BARTSCH, P., PRIGNOT, J. \& MARBAIX, S. (2009) Cost effectiveness of varenicline in Belgium, compared with bupropion, nicotine replacement therapy, brief counselling and unaided smoking cessation: a BENESCO Markov cost-effectiveness analysis. Clinical Drug Investigation, 29, 655-65.

APPLEGATE, B. W., SHEFFER, C. E., CREWS, K. M., PAYNE, T. J. \& SMITH, P. O. (2007) A survey of tobacco-related knowledge, attitudes and behaviours of primary care providers in Mississippi. Journal of Evaluation in Clinical Practice, 14, 537-544.

ARMITAGE, C. J. \& CONNER, M. (2001) Efficacy of the Theory of Planned Behaviour: a meta-analytic review. British Journal of Social Psychology, 40, 471-99.

BALMFORD, J. \& BORLAND, R. (2008) What does it mean to want to quit? Drug and Alcohol Review, 27, 21-7.

BALMFORD, J., BORLAND, R. \& BURNEY, S. (2010) The influence of having a quit date on prediction of smoking cessation outcome. Health Education Research, 25, 698-706.

BANDURA, A. (1986) Social Foundations Of Thought And Action: A cognitive social theory, Englewood Cliffs, New York, Pretince Hall.

BANDURA, A. (1991) Social Cognitive Theory of Self-Regulation. Organizational Behavior and Human Decision Processes, 50, 248-287. 
BANDURA, A. (1998) Health Promotion from the Perspective of Social Cognitive Theory. Psychology and Health, 13, 623-649.

BERG, C. J., AN, L. C., KIRCH, M., GUO, H., THOMAS, J. L., PATTEN, C. A., AHLUWALIA, J. S. \& WEST, R. (2010) Failure to report quit attempts: Implications for research and clinical practice. Addictive Behaviors, 35, 900-904.

BERNSTEIN, D. A., PENNER, L. A., CLARKE-STEWART, A. \& ROY, E. J. (2003) Psychology, Boston, Houghton Mifflin Company.

BLANKERS, M., KOETER, M. W. \& SCHIPPERS, G. M. (2010) Missing data approaches in eHealth research: simulation study and a tutorial for nonmathematically inclined researchers. Journal of Medical Internet Research, 12, e54.

BOLMAN, C., DE VRIES, H. \& MESTERS, I. (2002a) Factors determining cardiac nurses' intentions to continue using a smoking cessation protocol. Heart \& Lung, 31, 15-24.

BOLMAN, C., DE VRIES, H. \& VAN BREUKELEN, G. (2002b) Evaluation of a nurse-managed minimalcontact smoking cessation intervention for cardiac inpatients. Health Education Research, 17, 99-116.

BORGEN, F. H. \& BARNETT, D. C. (1987) Applying Cluster Analysis in Counselling Psychology Research. Journal of Counselling Psychology, 34, 456-468.

BORLAND, R., BALMFORD, J. \& HUNT, D. (2004) The effectiveness of personally tailored computergenerated advice letters for smoking cessation. Addiction, 99, 369-377.

BORLAND, R., YONG, H. H., BALMFORD, J., COOPER, J., CUMMINGS, K. M., O'CONNOR, R. J., MCNEILL, A., ZANNA, M. P. \& FONG, G. T. (2010) Motivational factors predict quit attempts but not maintenance of smoking cessation: findings from the International Tobacco Control Four country project. Nicotine and Tobacco Research, 12 Suppl, S4-11.

BOUMA, J., RANCHOR, A. V., SANDERMAN, R. \& SONDEREN, E., VAN (1995) Het meten van symptomen van depressie met de CES-D: een handleiding, Groningen, Noordelijk Centrum voor Gezondheidsvraagstukken, Rijksuniversiteit Groningen.

BOUTER, L. M., VAN DONGEN, M. \& ZIELHUIS, G. A. (2005) Epidemiologisch onderzoek: opzet en interpretatie, Centraal Boekhuis.

BROUWER, W., OENEMA, A., RAAT, H., CRUTZEN, R., DE NOOIJER, J., DE VRIES, N. K. \& BRUG, J. (2010) Characteristics of visitors and revisitors to an Internet-delivered computer-tailored lifestyle intervention implemented for use by the general public. Health Education Research, 25, 585-95.

BRUG, H., VAN ASSEMA, P. \& LECHNER, L. (2008) Gezondheidsvoorlichting en gedragsverandering: een planmatige aanpak, Assen, Van Gorcum.

BRUG, J., GLANZ, K., VAN ASSEMA, P., KOK, G. \& VAN BREUKELEN, G. J. P. (1998) The impact of computer-tailored feedback and iterative feedback on fat, fruit, and vegetable intake. Health Education Behaviour, 25, 517-531.

CAMPBELL, M., FITZPATRICK, R., HAINES, A., KINMONTH, A. L., SANDERCOCK, P., SPIEGELHALTER, D. \& TYRER, P. (2000) Framework for design and evaluation of complex interventions to improve health. British Medical Journal, 321, 694-6.

CASPARIE, A. F., VAN HOUT, B. A. \& SIMOONS, M. L. (1998) Richtlijnen en kosten. Nederlands Tijdschrift voor Geneeskunde, 142, 2075-7.

CENTRAAL BUREAU VOOR DE STATISTIEK. ICT gebruik van personen naar persoonskenmerken. Accessed: 201128 March; http://statline.cbs.nl/StatWeb/publication/?VW=T\&DM$=$ SLNL\&PA=71098ned\&LA=NL Archived by WebCite at: http://www.webcitation.org/690RVvulz

CENTRAAL BUREAU VOOR DE STATISTIEK. 1,2 miljoen Nederlanders hebben thuis geen internet. Accessed: 2009 December 22; http://www.cbs.nl/nl-NL/menu/themas/vrije-tijd-cultuur/publicaties/artikelen/archief/2008/2008-2641-wm.htm Archived by WebCite at: http://www.webcitation.org/5xLwWmphS 
CHARLTON, A. (2005) Tobacco or health 1602: an Elizabethan doctor speaks. Health Education Research, 20, 101-11.

CHAVANNES, N. H., KAPER, J., FRIJLING, B. D., VAN DER LAAN, J. R., JANSEN, P. W. M., GUERROUJ, S., DRENTHEN, A. J. M., BAX, W. \& WIND, L. A. (2007) NHG-Standaard Stoppen met roken. Huisarts \& Wetenschap, 50, 306-314.

CIVLJAK, M., SHEIKH, A., STEAD, L. F. \& CAR, J. (2010) Internet-based interventions for smoking cessation. Cochrane Database of Systematic Reviews, Cd007078.

CLARK, M. A., ROGERS, M. L., ARMSTRONG, G. F., RAKOWSKI, W. \& KVIZ, F. J. (2008) Differential response effects of data collection mode in a cancer screening study of unmarried women ages 40-75 years: a randomized trial. Medical Research Methodology, 8, 10.

COLEMAN, T., WYNN, A. T., STEVENSON, K. \& CHEATER, F. (2001) Qualitative study of pilot payment aimed at increasing general practitioners' antismoking advice to smokers. British Medical Journal, 323, 432-5.

COMMISSIE FARMACOTHERAPAUTISCHE HULP CVZ (2011) Farmacotherapeutisch Kompas: medisch farmaceutische voorlichting, Amstelveen, College voor zorgverzekeringen.

CRONBACH, L. J. \& GLESER, G. C. (1953) Assessing similarity between profiles. Psychology Bulletin, $50,456-73$.

CRUTZEN, R., DE NOOIJER, J., BROUWER, W., OENEMA, A., BRUG, J. \& DE VRIES, N. K. (2011) Strategies to facilitate exposure to internet-delivered health behavior change interventions aimed at adolescents or young adults: a systematic review. Health Education and Behavior, $38,49-62$.

CURRY, S., WAGNER, E. H. \& GROTHAUS, L. C. (1990) Intrinsic and extrinsic motivation for smoking cessation. Journal of Consulting and Clinical Psychology, 58, 310-6.

CURRY, S. J., GROTHAUS, L. \& MCBRIDE, C. (1997) Reasons for quitting: intrinsic and extrinsic motivation for smoking cessation in a population-based sample of smokers. Addictive Behaviors, 22, 727-39.

CURRY, S. J., MCBRIDE, C., GROTHAUS, L. C., LOUIE, D. \& WAGNER, E. H. (1995) A randomized trial of self-help materials, personalized feedback, and telephone counselling with nonvolunteer smokers. Journal of Consulting and Clinical Psychology, 63, 1005-1014.

DALSTRA, J. A., KUNST, A. E., GEURTS, J. J., FRENKEN, F. J. \& MACKENBACH, J. P. (2002) Trends in socioeconomic health inequalities in the Netherlands, 1981-1999. Journal of Epidemiology and Community Health, 56, 927-34.

DAMOISEAUX, V. M. G. \& VAN DER MOLEN, H. T. (1998) Gezondheidsvoorlichting en gedragsverandering, Assen, Van Gorcum.

DE BRUIN, M., VIECHTBAUER, W., HOSPERS, H. J., SCHAALMA, H. P. \& KOK, G. (2009) Standard care quality determines treatment outcomes in control groups of HAART-adherence intervention studies: implications for the interpretation and comparison of intervention effects. Health Psychology, 28, 668-74.

DE NOOIJER, J., OENEMA, A., KLOEK, G., BRUG, H., DE VRIES, H. \& DE VRIES, N. (2005) Bevordering van gezond gedrag via internet. Nu en in de toekomst, Maastricht, Maastricht University.

DE VET, E., DE NOOIJER, J., DE VRIES, N. K. \& BRUG, J. (2007) Comparing stage of change and behavioral intention to understand fruit intake. Health Education Research, 22, 599-608.

DE VRIES, H. \& BRUG, J. (1999) Computer-tailored interventions motivating people to adopt health promoting behaviours: introduction to a new approach. Patient Education and Counseling, 36, 99-105.

DE VRIES, H., EGGERS, M. \& BOLMAN, C. (2012) The role of action planning and enactment for smoking cessation. In preparation.

DE VRIES, H., MUDDE, A., LEIJS, I., CHARLTON, A., VARTIAINEN, E., BUIJS, G., CLEMENTE, M. P., STORM, H., GONZALEZ NAVARRO, A., NEBOT, M., PRINS, T. \& KREMERS, S. (2003) The European Smoking prevention Framework Approach (EFSA): an example of integral prevention. Health Education Research, 18, 611-626. 
DE VRIES, H. \& MUDDE, A. N. (1998) Predicting stage transitions for smoking cessation applying the attitude-social influence-efficacy model. Psychology \& Health, 13, 369-385.

DE VRIES, H., MUDDE, A. N., DIJKSTRA, A. \& WILLEMSEN, M. C. (1998) Differential beliefs, perceived social influences, and self-efficacy expectations among smokers in various motivational phases. Preventive Medicine, 27, 681-9.

DE VRIES, H., VAN 'T RIET, J., SPIGT, M., METSEMAKERS, J., VAN DEN AKKER, M., VERMUNT, J. K. \& KREMERS, S. (2008) Clusters of lifestyle behaviors: Results from the Dutch SMILE study. Preventive Medicine, 46, 203-208.

DECI, E. L. \& RYAN, R. M. (1988) Intrinsic motivation and self-determination in human behavior, New York, Plenum Press.

DECI, E. L. \& RYAN, R. M. (2008) Facilitating Optimal Motivation and Psychological Well-Being Across Life's Domains. Canadian Psychology, 49, 14-23.

DERCKX, E. (2006) Eerste nurse practitioners met differentiate huisartsenzorg. Tijdschrift voor Verpleegkundigen, 3, 26-30.

DICLEMENTE, C. C., PROCHASKA, J. O., FAIRHURST, S. K., VELICER, W. F., VELASQUEZ, M. M. \& ROSSI, J. S. (1991) The process of smoking cessation: an analysis of precontemplation, contemplation, and preparation stages of change. Journal of Consulting and Clinical Psychology, 59, 295-304.

DICLEMENTE, C. C., PROCHASKA, J. O. \& GIBERTINI, M. (1985) Self-Efficacy and the Stages of SelfChange of Smoking. Cognitive Therapy and Research, 9, 181-200.

DIJKSTRA, A. (2005) Working mechanisms of computer-tailored health education: evidence from smoking cessation. Health Education Research, 20, 527-39.

DIJKSTRA, A. (2008) The Psychology of Tailoring-Ingredients in Computer-Tailored Persuasion. Social and Personality Psychology Compass, 2, 765-784.

DIJKSTRA, A. \& BORLAND, R. (2003) Residual outcome expectations and relapse in ex-smokers. Health Psychology, 22, 340-6.

DIJKSTRA, A., CONIJN, B. \& DE VRIES, H. (2006) A match-mismatch test of a stage model of behaviour change in tobacco smoking. Addiction, 101, 1035-43.

DIJKSTRA, A. \& DE VRIES, H. (1999) The development of computer-generated tailored interventions. Patient Education and Counseling, 36, 193-203.

DIJKSTRA, A. \& DE VRIES, H. (2000) Clusters of precontemplating smokers defined by the perception of the pros, cons, and self-efficacy. Addictive Behaviors, 25, 373-385.

DIJKSTRA, A., DE VRIES, H. \& BAKKER, M. (1996) Pros and cons of quitting, self-efficacy, and the stages of change in smoking cessation. Journal of Consulting and Clinical Psychology, 64, 758763.

DIJKSTRA, A., DE VRIES, H. \& ROIJACKERS, J. (1998a) Long-term effectiveness of computergenerated tailored feedback in smoking cessation. Health Education Research, 13, 207-214.

DIJKSTRA, A., DE VRIES, H., ROIJACKERS, J. \& VAN BREUKELEN, G. (1998b) Tailored interventions to communicate stage-matched information to smokers in different motivational stages. Journal of Consulting and Clinical Psychology, 66, 549-557.

DIJKSTRA, A., DE VRIES, H., ROIJACKERS, J. \& VAN BREUKELEN, G. (1998c) Tailoring information to enhance quitting in smokers with low motivation to quit: Three basic efficacy questions. Health Psychology, 17, 513-519.

DIJKSTRA, A., ROIJACKERS, J. \& DE VRIES, H. (1998d) Smokers in four stages of readiness to change. Addictive Behaviors, 23, 339-350.

DOLL, R. \& HILL, A. B. (1956) Lung cancer and other causes of death in relation to smoking; a second report on the mortality of British doctors. British Medical Journal, 2, 1071-1081.

DRUMMOND, M. F. (1997) Methods for the economic evaluation of health care programmes, Oxford, Oxford University Press. 
DRUMMOND, M. F., O'BRIEN, B. J., SCULPHER, M. J., THORRANCE, G. W. \& STODDART, G. L. (2005) Methods for the economic evaluation of health care programmes, Oxford, Oxford University Press.

DSS RESEARCH. Researcher's Toolkit. Accessed: 2010 January 13; http://www.dssresearch.com/toolkit/sscalc/size.asp Archived by WebCite at: http://www.webcitation.org/5xLwjt2EA

ECCLES, M. P., HRISOS, S., FRANCIS, J., KANER, E. F., DICKINSON, H. O., BEYER, F. \& JOHNSTON, M. (2006) Do self- reported intentions predict clinicians' behaviour: a systematic review. Implementation Science, 1, 28.

ECCLES, M. P., JOHNSTON, M., HRISOS, S., FRANCIS, J., GRIMSHAW, J., STEEN, N. \& KANER, E. F. (2007) Translating clinicians' beliefs into implementation interventions (TRACII): a protocol for an intervention modeling experiment to change clinicians' intentions to implement evidence-based practice. Implementation Science, 2, 27.

ELFEDDALI, I., BOLMAN, C., CANDEL, M. J., WIERS, R. W. \& DE VRIES, H. (2012a) The role of selfefficacy, recovery self-efficacy, and preparatory planning in predicting short-term smoking relapse. British Journal of Health Psychology, 17, 185-201.

ELFEDDALI, I., BOLMAN, C. \& DE VRIES, H. (2012b) SQ4U - a computer tailored smoking relapse prevention program incorporating planning strategy assignments and multiple feedback time points after the quit-attempt: development and design protocol. Contemporary Clinical Trials, $33,151-8$.

ERLER, A., BODENHEIMER, T., BAKER, R., GOODWIN, N., SPREEUWENBERG, C., VRIJHOEF, H. J., NOLTE, E. \& GERLACH, F. M. (2011) Preparing primary care for the future - perspectives from the Netherlands, England, and USA. Zeitschrift fur Evident, Fortbildung und Qualitatssicherung Gesundheidswesen, 105, 571-80.

EROL, S. \& ERDOGAN, S. (2008) Application of a stage based motivational interviewing approach to adolescent smoking cessation: the Transtheoretical Model-based study. Patient Education and Counseling, 72, 42-48.

ETTER, J. F. \& PERNEGER, T. V. (2001a) A comparison of cigarette smokers recruited through the Internet or by mail. International Journal of Epidemiology, 30, 521-5.

ETTER, J. F. \& PERNEGER, T. V. (2001b) Effectiveness of a computer-tailored smoking cessation program: a randomized trial. Archives of Internal Medicine, 161, 2596-601.

EUROQOL GROUP (1990) EuroQol--a new facility for the measurement of health-related quality of life. The EuroQol Group. Health Policy, 16, 199-208.

EVERITT, B. (1980) Cluster Analysis, London, Heinemann Educational Books.

EVERS, S., WOLFS, C. \& VAN HEUGTEN, C. (2010) Economische evaluatie van neuropsychologische behandeling. IN PONDS, R., VAN HEUGTEN, C., FASOTTI, L. \& WEKKING, E. (Eds.) Neuropsychologische Behandeling. Amsterdam, Boom Uitgeverij.

EYSENBACH, G. (2005) The law of attrition. Journal of Medical Internet Research, 7, e11.

EYSENBACH, G. (2011) CONSORT-EHEALTH: improving and standardizing evaluation reports of Web-based and mobile health interventions. Journal of Medical Internet Research, 13, e126.

FAUL, F. \& ERDFELDER, E. (1992) GPOWER: A priori, post-hoc, and compromise power analyses for MS-DOS. Bonn, FRG: Bonn University, Dep. of Psychology.

FAVA, J. L., VELICER, W. F. \& PROCHASKA, J. O. (1995) Applying the transtheoretical model to a representative sample of smokers. Addictive Behaviors, 20, 189-203.

FIELD, A. Postgraduate Statistics: Cluster Analysis. Postgraduate Statistics Accessed: 2008 November 8; www.statisticshell.com/docs/cluster.pdf Archived by WebCite at: www.webcitation.org/68DVVTUDf

FIELD, A. (2005) Discovering statistics using SPSS, London, SAGE Publications Ltd.

FIORE, M. C. (2000) US public health service clinical practice guideline: treating tobacco use and dependence. Respiratory Care, 45, 1200-62.

FLAY, B. R. (1986) Efficacy and effectiveness trials (and other phases of research) in the development of health promotion programs. Preventive Medicine, 15, 451-74. 
FLAY, B. R., BIGLAN, A., BORUCH, R. F., CASTRO, F. G., GOTTFREDSON, D., KELLAM, S., MOSCICKI, E. K., SCHINKE, S., VALENTINE, J. C. \& JI, P. (2005) Standards of evidence: criteria for efficacy, effectiveness and dissemination. Preventive Science, 6, 151-75.

FLEUREN, M., WIEFFERINK, K. \& PAULUSSEN, T. (2004) Determinants of innovation within health care organizations: literature review and Delphi study. International Journal for Quality in Health Care, 16, 107-23.

FUCITO, L. M., LATIMER, A. E., CARLIN-MENTER, S., SAlOVEY, P., CUMMINGS, K. M., MAKUCH, R. W. \& TOLL, B. A. (2010) Nicotine dependence as a moderator of a quitline-based message framing intervention. Drug and Alcohol Dependence, 114, 229-32.

GAINSBURY, S. \& BLASZCZYNSKI, A. (2011) A systematic review of Internet-based therapy for the treatment of addictions. Clinical Psychology Review, 31, 490-8.

GERBING, D. W. \& ANDERSON, J. C. (1988) An Updated Paradigm for Scale Development Incorporating Unidimensionality and Its Assessment. Journal of Marketing Research, 25.

GERHARDS, S. A., DE GRAAF, L. E., JACOBS, L. E., SEVERENS, J. L., HUIBERS, M. J., ARNTZ, A., RIPER, H., WIDDERSHOVEN, G., METSEMAKERS, J. F. \& EVERS, S. M. (2010) Economic evaluation of online computerised cognitive-behavioural therapy without support for depression in primary care: randomised trial. British Journal of Psychiatry, 196, 310-8.

GIESBERS, H. Aantal huisartsen per 10.000 inwoners 2003. Volksgezondheid Toekomst Verkenning, Nationale Atlas Volksgezondheid. Accessed: 2009 December 23; http://www.rivm.nl/vtv/object_map/o420n21249.html Archived by WebCite at: http://www.webcitation.org/69B0sNn3X

GILBERT, H., LEURENT, B., SUTTON, S., MORRIS, R., ALEXIS GARSEE, C. \& NAZARETH, I. (2012) Factors predicting recruitment to a UK wide primary care smoking cessation study (the ESCAPE trial). Family Practice, 29, 110-7.

GILPIN, E. \& PIERCE, J. P. (1994) Measuring smoking cessation: problems with recall in the 1990 California Tobacco Survey. Cancer Epidemiology, Biomarkers and Prevention, 3, 613-7.

GLASGOW, R. E., LICHTENSTEIN, E. \& MARCUS, A. C. (2003) Why don't we see more translation of health promotion Research to Practice? Rethinking the Efficacy-to-Effectiveness Transition. American Journal of Public Health, 93, 1261-1267.

GLASGOW, R. E., VOGT, T. M. \& BOLES, S. M. (1999) Evaluating the public health impact of health promotion interventions: the RE-AIM framework. American Journal of Public Health, 89, 1322-7.

GLICK, H. A., DOSHI, J. A., SONNAD, S. S. \& POLSKY, D. (2007) Economic Evaluation in Clinical Trials, New York, Oxford University Press.

GODIN, G. \& KOK, G. (1996) The theory of planned behavior: a review of its applications to healthrelated behaviors. American Journal of Health Promotion, 11, 87-98.

GOLLWITZER, P. M. (1999) Implementation intentions. Strong effects of simple plans. American Psychologist, 54, 493-503.

GOLLWITZER, P. M. \& SHEERAN, P. (2006) Implementation Intentions and Goal Achievement: A Meta-analysis of effects and processes. Advances in Experimental Social Psychology, 38, 69119.

GOOSSENS, M. E., RUTTEN VAN MOLKEN, M. P., VLAEYEN, J. W. \& VAN DER LINDEN, S. M. (2000) The cost diary: a method to measure direct and indirect costs in cost-effectiveness research. Journal of Clinical Epidemiology, 53, 688-95.

GRAHAM, A. L., MILNER, P., SAUL, J. E. \& PFAFF, L. (2008) Online advertising as a public health and recruitment tool: comparison of different media campaigns to increase demand for smoking cessation interventions. Journal of Medical Internet Research, 10, e50.

GRIFFITHS, F., LINDENMEYER, A., POWELL, J., LOWE, P. \& THOROGOOD, M. (2006) Why are health care interventions delivered over the internet? A systematic review of the published literature. Journal of Medical Internet Research, 8, e10. 
GWALTNEY, C. J., METRIK, J., KAHLER, C. W. \& SHIFFMAN, S. (2009) Self-efficacy and smoking cessation: a meta-analysis. Psychology of Addictive Behaviors, 23, 56-66.

GWALTNEY, C. J., SHIFFMAN, S., BALABANIS, M. H. \& PATY, J. A. (2005) Dynamic self-efficacy and outcome expectancies: prediction of smoking lapse and relapse. Journal of Abnormal Psychology, 114, 661-75.

HAGGER, M. S. \& CHATZISARANTIS, N. L. (2008) Self-determination Theory and the psychology of exercise. International Review of Sport and Exercise Psychology, 1, 79-103.

HAGGER, M. S. \& CHATZISARANTIS, N. L. D. (2009) Integrating the theory of planned behaviour and self-determination theory in health behaviour: A meta-analysis. British Journal of Health Psychology, 14, 275-302.

HAJEK, P., STEAD, L. F., WEST, R., JARVIS, M. \& LANCASTER, T. (2009) Relapse prevention interventions for smoking cessation. Cochrane Database of Systematic Reviews, Cd003999.

HAKKAART-VAN ROIJEN, L., TAN, S. S. \& BOUWMANS, C. A. M. (2010) Handleiding voor kostenonderzoek, methoden en standaard kostprijzen voor economische evaluaties in de gezondheidszorg, College voor zorgverzekeringen.

HALL, S. \& MARTEAU, T. M. (2007) Practice nurses' self-reported opportunistic smoking cessation advice in three contexts. Nicotine and Tobacco Research, 9, 941-5.

HALL, S., VOGT, F. \& MARTEAU, T. M. (2005) A short report: survey of practice nurses' attitudes towards giving smoking cessation advice. Family Practice, 22, 614-6.

HAMMOND, D. (2011) Health warning messages on tobacco products: a review. Tobacco Control, 20, 327-37.

HARRINGTON, N. G. \& NOAR, S. M. (2012) Reporting standards for studies of tailored interventions. Health Education Research, 27, 331-42.

HAYS, J. T., CROGHAN, I. T., BAKER, C. L., CAPPELLERI, J. C. \& BUSHMAKIN, A. G. (2010) Changes in health-related quality of life with smoking cessation treatment. European Journal of Public Health.

HEATHER, N. (1991) Foreword. IN DAVIDSON, R., ROLLNICK, S. \& MACEWAN, I. (Eds.) Counselling problem drinkers. New York, Routledge.

HEATHERTON, T. F., KOZLOWSKI, L. T., FRECKER, R. C. \& FAGERSTROM, K. O. (1991) The Fagerstrom Test for Nicotine Dependence: a revision of the Fagerstrom Tolerance Questionnaire. British Journal of Addiction, 86, 1119-1127.

HECKMAN, C. J., EGLESTON, B. L. \& HOFMANN, M. T. (2010) Efficacy of motivational interviewing for smoking cessation: a systematic review and meta-analysis. Tobacco Control, 19, 410-6.

HELMINK, J. H., KREMERS, S. P., VAN BOEKEL, L. C., VAN BRUSSEL VISSER, F. N. \& DE VRIES, N. K. (2011) Factors determining the motivation of primary health care professionals to implement and continue the 'Beweegkuur' lifestyle intervention programme. Journal of Evaluation in Clinical Practice.

HENNINGFIELD, J. E. \& FAGERSTROM, K. O. (2001) Swedish Match Company, Swedish snus and public health: a harm reduction experiment in progress? Tobacco Control, 10, 253-257.

HERZOG, T. A. (2008) Analyzing the transtheoretical model using the framework of Weinstein, Rothman, and Sutton (1998): the example of smoking cessation. Health Psychology, 27, 548556.

HEYES, T., LONG, S. \& MATHERS, N. (2004) Preconception care, practice and beliefs of primary care workers. Family Practice, 21, 22-27.

HILBERINK, S. R., JACOBS, J. E., BRETELER, M. H., DE VRIES, H. \& GROL, R. P. (2011) General practice counselling for patients with chronic obstructive pulmonary disease to quit smoking: impact after 1 year of two complex interventions. Patient Education and Counseling, 83, 120-4.

HILL, J. (1761) Cautions against the immoderate use of stuff the effects it must produce when this way taken into the body, London, R. Baldwin and J. Jackson.

HINGSTMAN, L. \& KENENS, R. J. (2007) Cijfers uit registratie van huisartsen - peiling 2007 [Figures from GP registration, 2007]. Utrecht, NIVEL. 
HINGSTMAN, L. \& KENENS, R. J. (2010) Cijfers uit registratie van huisartsen - peiling 2010 [Figures from GP registration, 2010]. Utrecht, NIVEL.

HOOGENDOORN, M., FEENSTRA, T. L., HOOGENVEEN, R. T. \& RUTTEN VAN MOLKEN, M. P. (2010) Long-term effectiveness and cost-effectiveness of smoking cessation interventions in patients with COPD. Thorax, 65, 711-8.

HOPKINS, D. P., BRISS, P. A., RICARD, C. J., HUSTEN, C. G., CARANDE KULIS, V. G., FIELDING, J. E., ALAO, M. O., MCKENNA, J. W., SHARP, D. J., HARRIS, J. R., WOOLLERY, T. A. \& HARRIS, K. W. (2001) Reviews of evidence regarding interventions to reduce tobacco use and exposure to environmental tobacco smoke. American Journal of Preventive Medicine, 20, 16-66.

HOVING, C. (2007) The feasibility of a smoking cessation computer tailored expert system in Dutch general practice and community pharmacy setting. Department of Health Education and Health Promotion. Maastricht, Maastricht University.

HOVING, C., MUDDE, A. N. \& DE VRIES, H. (2006a) Intention to adopt a smoking cessation expert system within a self-selected sample of Dutch general practitioners. European Journal of Cancer Prevention, 15, 82-6.

HOVING, C., MUDDE, A. N. \& DE VRIES, H. (2007a) Effect of recruitment method and setting on the composition of samples consisting of adult smokers. Patient Education and Counseling, 65, 79-86.

HOVING, C., MUDDE, A. N. \& DE VRIES, H. (2007b) Intention to implement a smoking cessation expert system in Dutch general practice. Health Education, 107, 307-315.

HOVING, C., MUDDE, A. N., DIJK, F. \& DE VRIES, H. (2010) Effectiveness of a computer-generated tailored smoking cessation expert system in Dutch community pharmacy and general practice setting. Health Education, 110, 17-29.

HOVING, E. F., MUDDE, A. N. \& DE VRIES, H. (2006b) Predictors of smoking relapse in a sample of Dutch adult smokers; the roles of gender and action plans. Addictive Behaviors, 31, 11771189.

HOVING, E. F., MUDDE, A. N. \& DE VRIES, H. (2006c) Smoking and the 0 pattern; predictors of transitions through the stages of change. Health Education Research, 21, 305-314.

HUGHES, J. R., KEELY, J. \& NAUD, S. (2004) Shape of the relapse curve and long-term abstinence among untreated smokers. Addiction, 99, 29-38.

HUGHES, J. R., KEELY, J. P., NIAURA, R. S., OSSIP KLEIN, D. J., RICHMOND, R. L. \& SWAN, G. E. (2003) Measures of abstinence in clinical trials: issues and recommendations. Nicotine and Tobacco Research, 5, 13-25.

HYLAND, A., BORLAND, R., LI, Q., YONG, H. H., MCNEILL, A., FONG, G. T., O'CONNOR, R. J. \& CUMMINGS, K. M. (2006) Individual-level predictors of cessation behaviours among participants in the International Tobacco Control (ITC) Four Country Survey. Tobacco Control, 15 Suppl 3, iii83-94.

INTERNET WORLD STATS. Internet usage statistics: The internet big picture. Accessed: 2010 September 17; http://www.internetworldstats.com/stats.htm Archived by WebCite at: http://www.webcitation.org/5xLwg1Msm

JACOBS, N., DROST, R., AMENT, A., EVERS, S. \& CLAES, N. (2011a) Willingness to pay for a cardiovascular prevention program in highly educated adults: a randomized controlled trial. International Journal of Technology Assessment in Health Care, 27, 283-9.

JACOBS, N., HAGGER, M. S., STREUKENS, S., DE BOURDEAUDHUIJ, I. \& CLAES, N. (2011b) Testing an integrated model of the theory of planned behaviour and self-determination theory for different energy balance-related behaviours and intervention intensities. British Journal of Health Psychology, 16, 113-34.

JANZ, N. K., CHAMPION, V. L. \& STRECHER, V. J. (2002) The Health Belief Model. IN GLANZ, K. (Ed.) Health Behavior and Health Education: Theory, Research, and Practice. San Francisco, JosseyBass. 
JOHNSON, S. S., DRISKELL, M. M., JOHNSON, J. L., PROCHASKA, J. M., ZWICK, W. \& PROCHASKA, J. O. (2006) Efficacy of a transtheoretical model-based expert system for antihypertensive adherence. Disease Management, 9, 291-301.

JOHNSON, S. S., PAIVA, A. L., CUMMINS, C. O., JOHNSON, J. L., DYMENT, S. J., WRIGHT, J. A., PROCHASKA, J. O., PROCHASKA, J. M. \& SHERMAN, K. (2008) Transtheoretical model-based multiple behavior intervention for weight management: effectiveness on a population basis. Preventive Medicine, 46, 238-246.

JOHNSTON, J. M., CHAN, S. S. C., CHAN, S. S. K., LAM, T. H., CHI, I. \& LEUNG, G. M. (2004) Training nurses and social workers in smoking cessation counselling: a population needs assessment in Hong Kong. Preventive Medicine, 40, 389-406.

KANER, E. F., BEYER, F., DICKINSON, H. O., PIENAAR, E., CAMPBELL, F., SCHLESINGER, C., HEATHER, N., SAUNDERS, J. \& BURNAND, B. (2007) Effectiveness of brief alcohol interventions in primary care populations. Cochrane Database of Systematic Reviews, Cd004148.

KAPER, J., WAGENA, E. J., VAN SCHAYCK, C. P. \& SEVERENS, J. L. (2006) Encouraging smokers to quit: the cost effectiveness of reimbursing the costs of smoking cessation treatment. Pharmacoeconomics, 24, 453-64.

KASSEL, J. D., STROUD, L. R. \& PARONIS, C. A. (2003) Smoking, stress, and negative affect: correlation, causation, and context across stages of smoking. Psychology Bulletin, 129, 270304.

KEATING, G. M. \& SIDDIQUI, M. A. (2006) Varenicline: a review of its use as an aid to smoking cessation therapy. CNS Drugs, 20, 945-60.

KHADJESARI, Z., MURRAY, E., KALAITZAKI, E., WHITE, I. R., MCCAMBRIDGE, J., G., T. S., P., W. \& GODFREY, C. (2011) Impact and Costs of Incentives to Reduce Attrition in Online Trials: Two Randomized Controlled Trials. Journal of Medical Internet Research, 13, e26.

KILLEN, J. D. \& FORTMANN, S. P. (1997) Craving is associated with smoking relapse: findings from three prospective studies. Experimental and Clinical Psychopharmacology, 5, 137-42.

KREBS, P., PROCHASKA, J. O. \& ROSSI, J. S. (2010) A meta-analysis of computer-tailored interventions for health behavior change. Preventive Medicine, 51, 214-21.

KREUTER, M., FARRELL, D., OLEVITCH, L. \& BRENNAN, L. (1999) Tailoring Health Messages: Customizing Communication with Computer Technology, Lawrence Erlbaum Associates.

KWALITEITSINSTITUUT VOOR DE GEZONDHEIDSZORG CBO (2004) Richtlijn Behandeling van tabaksverslaving [Directive Treatment of tobacco addiction]. Kwaliteitsinstituut voor de Gezondheidszorg CBO.

LAMBERT, M. T., TERREL, J. E., COPELAND, L. A., RONIS, D. L. \& DUFFY, S. A. (2005) Cigarettes, alcohol and depression: characterizing head and neck cancer survivors in two systems of care. Nicotine and Tobacco Research, 7, 233-241.

LAMERS, L. M., MCDONNELL, J., STALMEIER, P. F., KRABBE, P. F. \& BUSSCHBACH, J. J. (2006) The Dutch tariff: results and arguments for an effective design for national EQ-5D valuation studies. Health Economics, 15, 1121-32.

LAMKADDEM, M., DE BAKKER, D., NIJLAND, A. \& DE HAAN, J. (2004) De invloed van praktijkondersteuning op de werklast van huisartsen [The influence of general practice support on the GP's workload]. Utrecht, NIVEL.

LANCASTER, T. \& STEAD, L. (2005a) Physician advice for smoking cessation. Cochrane Database of Systematic Reviews, Cd000165.

LANCASTER, T. \& STEAD, L. (2008) Physician advice for smoking cessation. Cochrane Database of Systematic Reviews, Cd000165.

LANCASTER, T., STEAD, L., SILAGY, C. \& SOWDEN, A. (2000) Effectiveness of interventions to help people stop smoking: findings from the Cochrane Library. British Medical Journal, 321, 355358.

LANCASTER, T. \& STEAD, L. F. (2005b) Individual behavioural counselling for smoking cessation. Cochrane Database of Systematic Reviews, Cd001292. 
LANCASTER, T. \& STEAD, L. F. (2005c) Self-help interventions for smoking cessation. Cochrane Database of Systematic Reviews, 1-91.

LAURANT, M., HERMENS, R., BRASPENNING, J. \& GROL, R. (2008) Praktijk ondersteuning verbetert zorg maar verlaagt werkdruk niet. Tijdschrift voor Praktijkondersteuning, 3, 126-130.

LAWHON, D., HUMFLEET, G. L., HALL, S. M., MUNOZ, R. F. \& REUS, V. I. (2009) Longitudinal analysis of abstinence-specific social support and smoking cessation. Health Psychology, 28, 465-72.

LEE, T. T. (2004) Nurses' adoption of technology: application of Rogers' innovation-diffusion model. Applied Nursing Research, 17, 231-8.

LEITLEIN, L., SMIT, E. S., DE VRIES, H. \& HOVING, C. (2011) Factors influencing Dutch practice nurses' intention to adopt a new smoking cessation intervention. Journal of Advanced Nursing, in press.

LEMMENS, V., OENEMA, A., KNUT, I. K. \& BRUG, J. (2008) Effectiveness of smoking cessation interventions among adults: a systematic review of reviews. European Journal of Cancer Prevention, 17, 535-44.

LEVENTHAL, A. M., RAMSEY, S. E., BROWN, R. A., LACHANCE, H. R. \& KAHLER, C. W. (2008) Dimensions of depressive symptoms and smoking cessation. Nicotine and Tobacco Research, $10,507-17$.

LEVY, D. T., CHALOUPKA, F. \& GITCHELL, J. (2004) The effects of tobacco control policies on smoking rates: a tobacco control scorecard. Journal of Public Health Management Practice, 10, 338-53.

LINDSTROM, D., SUNDBERG PETERSSON, I., ADAMI, J. \& TONNESEN, H. (2009) Disappointment and drop-out rate after being allocated to control group in a smoking cessation trial. Contemporary Clinical Trials, 31, 22-6.

LITTEL, J. H. \& GIRVIN, H. (2002) Stages of change: A Critique. Behavior Modification, 26, 223-273.

LORENCE, D. \& PARK, H. (2008) Group disparities and health information: a study of online access for the underserved. Health Informatics J, 14, 29-38.

LUSTRIA, M. L., CORTESE, J., NOAR, S. M. \& GLUECKAUF, R. L. (2009) Computer-tailored health interventions delivered over the Web: review and analysis of key components. Patient Education and Counseling, 74, 156-73.

MARKLAND, D., RYAN, R. M., TOBIN, V. J. \& ROLLNICK, S. (2005) Motivational Interviewing and SelfDetermination Theory. Journal of Social and Clinical Psychology, 24, 811-831.

MARMOT, M. (2005) Social determinants of health inequalities. Lancet, 365, 1099-104.

MCAVOY, B. R., KANER, E. F., LOCK, C. A., HEATHER, N. \& GILVARRY, E. (1999) Our Healthier Nation: are general practitioners willing and able to deliver? A survey of attitudes to and involvement in health promotion and lifestyle counselling. British Journal of General Practice, 49, 187-90.

MCCARTY, M. C., HENNRIKUS, D. J., LANDO, H. A. \& VESEY, J. T. (2001) Nurses' attitudes concerning the delivery of brief cessation advice to hospitalized smokers. Preventive Medicine, 33, 674681.

MCEWEN, A. \& WEST, R. (2001) Smoking cessation activities by general practitioners and practice nurses. Tobacco Control, 10, 27-32.

MCEWEN, A., WEST, R. \& MCROBBIE, H. (2008) Motives for smoking and their correlates in clients attending Stop Smoking treatment services. Nicotine and Tobacco Research, 10, 843-50.

MCGUIRE, W. J. (1985) Attitudes and attitude change. Handbook of Social psychology. New York, Lawrence Erlbaum Associates.

MCKAY, H. G., DANAHER, B. G., SEELEY, J. R., LICHTENSTEIN, E. \& GAU, J. M. (2008) Comparing two web-based smoking cessation programs: randomized controlled trial. Journal of Medical Internet Research, 10, e40.

MEINSMA, L. (1969) Roken en risico's, Lochem, the Netherlands, De Tijdstroom.

MEYER, C., ULBRICHT, S., BAUMEISTER, S. E., SCHUMANN, A., RUGE, J., BISCHOF, G., RUMPF, H. J. \& JOHN, U. (2008) Proactive interventions for smoking cessation in general medical practice: a 
quasi-randomized controlled trial to examine the efficacy of computer-tailored letters and physician-delivered brief advice. Addiction, 103, 294-304.

MILLER, W. R. \& ROLLNICK, S. (1991) Motivational interviewing: Preparing people to change addictive behavior, New York, Guilford.

MILLER, W. R. \& ROLLNICK, S. (2009) Ten Things that Motivational Interviewing Is Not. Behavioural and Cognitive Psychotherapy, 37, 129-140.

MILLS, A. L., MESSER, K., GILPIN, E. A. \& PIERCE, J. P. (2009) The effect of smoke-free homes on adult smoking behavior: a review. Nicotine and Tobacco Research, 11, 1131-41.

MOHER, M., HEY, K. \& LANCASTER, T. (2003) Workplace interventions for smoking cessation. Cochrane Database of Systematic Reviews, Cd003440.

MUDDE, A. N., WILLEMSEN, M. C., KREMERS, S. \& VRIES, H., DE (2006) Meetinstrumenten: voor onderzoek naar roken en stoppen met roken, Den Haag, STIVORO voor een rookvrije toekomst.

MUENNIG, P., FISCELLA, K., TANCREDI, D. \& FRANKS, P. (2010) The relative health burden of selected social and behavioral risk factors in the United States: implications for policy. American Journal of Public Health, 100, 1758-64.

NAGELHOUT, G. E. (2007) Belemmerende en bevorderende factoren voor het bereik en de toepassing van de richtlijn 'Behandeling van tabaksverslaving' bij oncologen en ondersteunende zorgverleners in de huisartsenpraktijk. Communication Studies. Twente, Universiteit Twente.

NAGELHOUT, G. E., WILLEMSEN, M. C. \& DE VRIES, H. (2011) The population impact of smoke-free workplace and hospitality industry legislation on smoking behaviour. Findings from a national population survey. Addiction, 106, 816-23.

NARBRO, K. \& SJOSTROM, L. (2000) Willingness to pay for obesity treatment. International Journal of Technology Assessment in Health Care, 16, 50-9.

NEDERLANDS HUISARTSEN GENOOTSCHAP \& LANDELIJKE HUISARTSEN VERENIGING (2011) NHG/LHV-Standpunt: Het (ondersteunend) team in de huisartsenvoorziening. Utrecht.

NEEDLEMAN, I., WARNAKULASURIYA, S., SUTHERLAND, G., BORNSTEIN, M. M., CASALS, E., DIETRICH, T. \& SUVAN, J. (2006) Evaluation of tobacco use cessation (TUC) counselling in the dental office. Oral Health and Preventive Dentistry, 4, 27.

NIEMIEC, C. P., RYAN, R. M., DECI, E. L. \& WILLIAMS, G. (2009) Aspiring to physical health: the role of aspirations for physical health in facilitating long-term tobacco abstinence. Patient Education and Counseling, 74, 250-257.

NOAR, S. M., BENAC, C. N. \& HARRIS, M. S. (2007) Does tailoring matter? Meta-analytic review of tailored print health behavior change interventions. Psychology Bulletin, 133, 673-93.

NORMAN, G. J., VELICER, W. F., FAVA, J. L. \& PROCHASKA, J. O. (2000) Cluster subtypes within stage of change in a representative sample of smokers. Addictive Behaviors, 25, 183-204.

NORMAN, P., CONNER, M. \& BELL, R. (1999) The theory of planned behavior and smoking cessation. Health Psychology, 18, 89-94.

O'CONNELL, K. A., HOSEIN, V. L., SCHWARTZ, J. E. \& LEIBOWITZ, R. Q. (2007) How does coping help people resist lapses during smoking cessation? Health Psychology, 26, 77-84.

OCKENE, J. K., EMMONS, K. M., MERMELSTEIN, R. J., PERKINS, K. A., BONOLLO, D. S., VOORHEES, C. C. \& HOLLIS, J. F. (2000) Relapse and maintenance issues for smoking cessation. Health Psychology, 19, 17-31.

ORBELL, S., LIDIERTH, P., HENDERSON, C. J., GEERAERT, N., ULLER, C., USKUL, A. K. \& KYRIAKAKI, M. (2009) Social-cognitive beliefs, alcohol, and tobacco use: a prospective community study of change following a ban on smoking in public places. Health Psychology, 28, 753-61.

PANDAY, S., REDDY, S. P., RUITER, R. A., BERGSTROM, E. \& DE VRIES, H. (2007) Determinants of smoking among adolescents in the Southern Cape-Karoo region, South Africa. Health Promotion International, 22, 207-17.

PARTNERSHIP STOP MET ROKEN (2009) Richtlijn Behandeling van tabaksverslaving herziening 2009. Den Haag. 
PATRICK, D. L., CHEADLE, A., THOMPSON, D. C., DIEHR, P., KOEPSELL, T. \& KINNE, S. (1994) The validity of self-reported smoking: a review and meta-analysis. American Journal of Public Health, 84, 1086-1093.

PIASECKI, T. M., NIAURA, R., SHADEL, W. G., ABRAMS, D., GOLDSTEIN, M., FIORE, M. C. \& BAKER, T. B. $(2000)$ Smoking withdrawal dynamics in unaided quitters. Journal of Abnormal Psychology, $109,74-86$.

PIETERSE, M. E., SEYDEL, E. R., DEVRIES, H., MUDDE, A. N. \& KOK, G. J. (2001) Effectiveness of a minimal contact smoking cessation program for Dutch general practitioners: a randomized controlled trial. Preventive Medicine, 32, 182-90.

POSTEL, M. G., DE HAAN, H. A., TER HUURNE, E. D., VAN DER PALEN, J., BECKER, E. S. \& DE JONG, C. A. J. (2011) Attrition in Web-Based Treatment for Problem Drinkers. Journal of Medical Internet Research, 13, e117.

PRESTON, C. C. \& COLMAN, A. M. (2000) Optimal number of response categories in rating scales: reliability, validity, discriminating power, and respondent preferences. Acta Psychologica (Amsterdam), 104,1-15.

PROCHASKA, J. O., BUTTERWORTH, S., REDDING, C. A., BURDEN, V., PERRIN, N., LEO, M., FLAHERTY ROBB, M. \& PROCHASKA, J. M. (2008) Initial efficacy of MI, TTM tailoring and HRI's with multiple behaviors for employee health promotion. Preventive Medicine, 46, 226-31.

PROCHASKA, J. O. \& DICLEMENTE, C. C. (1983) Stages and processes of self-change of smoking: Toward an integrative model of change. Journal of Consulting and Clinical Psychology, 51, 390395.

PROCHASKA, J. O., REDDING, C. A. \& EVERS, K. E. (1997) The Transtheoretical Model and Stages of Change. IN GLANZ, K. (Ed.) Health Behavior and Health Education: Theory, Research, and Practice. San Francisco, Jossey-Bass.

PROCHASKA, J. O., VELICER, W. F., GUADAGNOLI, E., ROSSI, J. S. \& DICLEMENTE, C. C. (1991) Patterns of Change: Dynamic Typology Applied to Smoking Cessation Multivariate Behavioral Research, 26, 83 - 107.

PROCHASKA, J. O., VELICER, W. F., REDDING, C., ROSSI, J. S., GOLDSTEIN, M., DEPUE, J., GREENE, G. W., ROSSI, S. R., SUN, X., FAVA, J. L., LAFORGE, R., RAKOWSKI, W. \& PLUMMER, B. A. (2005) Stage-based expert systems to guide a population of primary care patients to quit smoking, eat healthier, prevent skin cancer, and receive regular mammograms. Preventive Medicine, 41, 406-416.

PROCHASKA, J. O., VELICER, W. F., ROSSI, J. S., GOLDSTEIN, M. G., MARCUS, B. H., RAKOWSKI, W., FIORE, C., HARLOW, L. L., REDDING, C. A., ROSENBLOOM, D. \& ET AL. (1994) Stages of change and decisional balance for 12 problem behaviors. Health Psychology, 13, 39-46.

PRONK, M. C., BLOM, A. T., JONKERS, R. \& VAN BURG, A. (2001) The diffusion process of patient education in Dutch community pharmacy: an exploration. Patient Education and Counseling, $42,115-21$.

PUFFER, S. \& RASHIDIAN, A. (2004) Practice nurses' intentions to use clinical guidelines. Journal of Advanced Nursing, 47, 500-9.

RAAD VOOR DE VOLKSGEZONDHEID EN ZORG (2006) Zinnige en duurzame zorg. Zoetermeer.

RABOIS, D. \& HAAGA, D. A. (2003) The influence of cognitive coping and mood on smokers' selfefficacy and temptation. Addictive Behaviors, 28, 561-73.

RASBASH, J., STEELE, F., BROWNE, W. J. \& GOLDSTEIN, H. (2009) A User's Guide to MLwiN, v2.10, Centre for Multilevel Modelling, University of Bristol.

REDA, A. A., KAPER, J., FIKRELTER, H., SEVERENS, J. L. \& VAN SCHAYCK, C. P. (2009) Healthcare financing systems for increasing the use of tobacco dependence treatment. Cochrane Database of Systematic Reviews, Cd004305.

RICE, V. H. \& STEAD, L. F. (2008) Nursing interventions for smoking cessation. Cochrane Database of Systematic Reviews, Cd001188.

ROGERS, E. M. (1995) Diffusion of Innovations, New York, Free Press. 
ROGERS, E. M. (2002) Diffusion of preventive innovations. Addictive Behaviors, 27, 989-993.

ROME, A., PERSSON, U., EKDAHL, C. \& GARD, G. (2010) Willingness to pay for health improvements of physical activity on prescription. Scandinavian Journal of Public Health, 38, 151-9.

ROSNOW, R. L. \& ROSENTHAL, R. (1989) Statistical Procedures and the Justification of Knowledge in Psychological Science. American Psychologist, 44, 1276-1284.

ROSSITER, J. R. \& PERCY, L. (1987) Advertising and promotion management, New York, NY, McGrawHill.

RUTTEN, G., KREMERS, S., RUTTEN, S. \& HARTING, J. (2009) A theory-based cross-sectional survey demonstrated the important role of awareness in guideline implementation. Journal of Clinical Epidemiology, 62, 167-176.e1.

RYAN, R. M. \& DECI, E. L. (2000) Self-determination theory and the facilitation of intrinsic motivation, social development, and well-being. The American Psychologist, 55, 68-78.

SALES, M. P., OLIVEIRA, M. I., MATTOS, I. M., VIANA, C. M. \& PEREIRA, E. D. (2009) The impact of smoking cessation on patient quality of life. Jornal Brasileiro de pneumologia, 35, 436-41.

SALIZE, H. J., MERKEL, S., REINHARD, I., TWARDELLA, D., MANN, K. \& BRENNER, H. (2009) Costeffective primary care-based strategies to improve smoking cessation: more value for money. Archives of Internal Medicine, 169, 230-5; discussion 235-6.

SALLIS, J. F., OWEN, N. \& FISHER, E. B. (2002) Ecological Models of Health Behavior. IN GLANZ, K., RIMER, B. K. \& LEWIS, F. M. (Eds.) Health Behavior and Health Education: Theory, Research, and Practice. San Francisco, CA, Jossey-Bass.

SARNA, L., WEVERS, M. E., BROWN, J. K., LILLINGTON, L. \& BRECHT, M. L. (2001) Barriers to tobacco cessation in clinical practice: Report from a national srvey of oncology nurses. Nurse Outlook, 49, 166-172.

SCARBOROUGH, P., BHATNAGAR, P., WICKRAMASINGHE, K. K., ALLENDER, S., FOSTER, C. \& RAYNER, M. (2011) The economic burden of ill health due to diet, physical inactivity, smoking, alcohol and obesity in the UK: an update to 2006-07 NHS costs. Journal of Public Health.

SCHELLEVIS, F. G., WESTERT, G. P. \& DE BAKKER, D. H. (2005) The actual role of general practice in the dutch health-care system. Results of the second dutch national survey of general practice. Journal of Public Health, 13, 265-296.

SCHERS, H., BOR, H., VAN DEN HOOGEN, H. \& VAN WEEL, C. (2008) What went and what came? Morbidity trends in general practice from the Netherlands. European Journal of General Practice, 14 Suppl 1, 13-24.

SCHIPPERS, E. I. (2011) Landelijke nota gezondheidsbeleid 'Gezondheid dichtbij' .

SCHOLZ, U., NAGY, G., GOHNER, W., LUSZCZYNSKA, A. \& KLIEGEL, M. (2009) Changes in selfregulatory cognitions as predictors of changes in smoking and nutrition behaviour. Psychology \& Health, 24, 545-61.

SCHULZ, K. F., ALTMAN, D. G. \& MOHER, D. (2010) CONSORT 2010 Statement: updated guidelines for reporting parallel group randomised trials. Trials, 11, 32.

SCHWARZER, R. Modeling health behavior change: The Health Action Process Approach (HAPA). Accessed: 2008 April 4; http://userpage.fu-berlin.de/ health/hapa.htm Archived by WebCite at: http://www.webcitation.org/62uoShUrm

SCIENTIFIC ADVISORY COMMITTEE OF THE MEDICAL OUTCOMES TRUST (2002) Assessing health status and quality-of-life instruments: attributes and review criteria. Quality of Life Research, 11, 193-205.

SEGAAR, D., BOLMAN, C., WILLEMSEN, M. C. \& VRIES, H. (2006) Determinants of adoption of cognitive behavioral interventions in a hospital setting: example of a minimal-contact smoking cessation intervention for cardiology wards. Patient Education and Counseling, 61, 262-71. 
SEGAAR, D., WILLEMSEN, M. C., BOLMAN, C. \& DE VRIES, H. (2007) Nurse adherence to a minimalcontact smoking cessation intervention on cardiac wards. Research in Nursing \& Health, 30, 429-44.

SHAHAB, L. \& MCEWEN, A. (2009) Online support for smoking cessation: a systematic review of the literature. Addiction, 104, 1792-804.

SHIFFMAN, S. (2005) Dynamic influences on smoking relapse process. Journal of Personality, 73, 1715-48.

SHIFFMAN, S., BALABANIS, M. H., GWALTNEY, C. J., PATY, J. A., GNYS, M., KASSEL, J. D., HICKCOX, M. \& PATON, S. M. (2007) Prediction of lapse from associations between smoking and situational antecedents assessed by ecological momentary assessment. Drug and Alcohol Dependence, 91, 159-68.

SHIFFMAN, S., PATTEN, C., GWALTNEY, C., PATY, J., GNYS, M., KASSEL, J., HICKCOX, M., WATERS, A. \& BALABANIS, M. (2006) Natural history of nicotine withdrawal. Addiction, 101, 1822-32.

SHUM, C., HUMPHREYS, A., WHEELER, D., COCHRANE, M. A., SKODA, S. \& CLEMENT, S. (2000) Nurse management of patients with minor illnesses in general practice: multicentre, randomised controlled trial. British Medical Journal, 320, 1038-43.

SIAHPUSH, M., MCNEILL, A., BORLAND, R. \& FONG, G. T. (2006) Socioeconomic variations in nicotine dependence, self-efficacy, and intention to quit across four countries: findings from the International Tobacco Control (ITC) Four Country Survey. Tobacco Control, 15 Suppl 3, iii71-5.

SINCLAIR, J., LAWSON, B. \& BURGE, J. (2008) Which patients receive on diet and exercise?: Do certain characteristics affect whether they receive such advice? Canadian Family Physician, $54,404-412$.

SMIT, E. S., DE VRIES, H. \& HOVING, C. (2010) The PAS study: a randomized controlled trial evaluating the effectiveness of a web-based multiple tailored smoking cessation programme and tailored counselling by practice nurses. Contemporary Clinical Trials, 31, 251-8.

SMIT, F., LOKKERBOL, J., RIPER, H., MAJO, M. C., BOON, B. \& BLANKERS, M. (2011) Modeling the cost-effectiveness of health care systems for alcohol use disorders: how implementation of eHealth interventions improves cost-effectiveness. Journal of Medical Internet Research, 13, e56.

SMITH, M. Y., CROMWELL, J., DEPUE, J., SPRING, B., REDD, W. \& UNROD, M. (2007) Determining the cost-effectiveness of a computer-based smoking cessation intervention in primary care. Managed Care, 16, 48-55.

SNIEHOTTA, F. F. (2009) Towards a theory of intentional behaviour change: plans, planning, and self-regulation. British Journal of Health Psychology, 14, 261-73.

STEAD, L. F., BERGSON, G. \& LANCASTER, T. (2008) Physician advice for smoking cessation. Cochrane Database of Systematic Reviews, Cd000165.

STEAD, M., ANGUS, K., HOLME, I., COHEN, D. \& TAIT, G. (2009) Factors influencing European GPs' engagement in smoking cessation: a multi-country literature review. British Journal of General Practice, 59, 682-90.

STEPTOE, A., DOHERTY, S., RINK, E., KERRY, S., KENDRICK, T. \& HILTON, S. (1999) Behavioural counselling in general practice for the promotion of healthy behaviour among adults at increased risk of coronary heart disease: randomised trial. British Medical Journal, 319, 9437; discussion 947-8.

STINNETT, A. A. \& MULLAHY, J. (1998) Net health benefits: a new framework for the analysis of uncertainty in cost-effectiveness analysis. Medical Decision Making, 18, S68-80.

STIVORO (2010) Percentage rokers Nederlandse bevolking 1958 - 2010. Den Haag, STIVORO - voor een rookvrije toekomst.

STRECHER, V. J. (1999) Computer-tailored smoking cessation materials: A review and discussion. Patient Education and Counseling, 36, 107-117. 
STRECHER, V. J., MCCLURE, J., ALEXANDER, G., CHAKRABORTY, B., NAIR, V., KONKEL, J., GREENE, S., COUPER, M., CARLIER, C., WIESE, C., LITTLE, R., POMERLEAU, C. \& POMERLEAU, O. (2008) The role of engagement in a tailored web-based smoking cessation program: randomized controlled trial. Journal of Medical Internet Research, 10, e36.

STRECHER, V. J., SHIFFMAN, S. \& WEST, R. (2006) Moderators and mediators of a web-based computer-tailored smoking cessation program among nicotine patch users. Nicotine and Tobacco Research, 8 Suppl 1, S95-101.

SUTTON, S. (2001) Back to the drawing board? A review of applications of the transtheoretical model to substance use. Addiction, 96, 175-186.

SUTTON, S. \& GILBERT, H. (2007) Effectiveness of individually tailored smoking cessation advice letters as an adjunct to telephone counselling and generic self-help materials: randomized controlled trial. Addiction, 102, 994-1000.

TAYLOR, S. E., KLEIN, L. C., LEWIS, B. P., GRUENEWALD, T. L., GURUNG, R. A. \& UPDEGRAFF, J. A. (2000) Biobehavioral responses to stress in females: tend-and-befriend, not fight-or-flight. Psychology Review, 107, 411-29.

TE POEL, F., BOLMAN, C., REUBSAET, A. \& DE VRIES, H. (2009) Efficacy of a single computertailored e-mail for smoking cessation: results after 6 months. Health Education Research, 24, 930-940.

TE VELDE, S. J., LENNERT VEERMAN, J., TAK, N. I., BOSMANS, J. E., KLEPP, K. I. \& BRUG, J. (2011) Modeling the long term health outcomes and cost-effectiveness of two interventions promoting fruit and vegetable intake among schoolchildren. Economy of Human Biology, 9, $14-22$.

TERWEE, C. B., BOT, S. D., DE BOER, M. R., VAN DER WINDT, D. A., KNOL, D. L., DEKKER, J., BOUTER, L. M. \& DE VET, H. C. (2007) Quality criteria were proposed for measurement properties of health status questionnaires. Journal of Clinical Epidemiology, 60, 34-42.

THE NHS INFORMATION CENTRE (2009) Statistics on Smoking, England 2009.

ULBRIGHT, S., MEYER, C., SCHUMANN, A., RUMPF, H. J., HAPKE, U. \& JOHN, U. (2006) Provision of smoking cessation counselling by general practitioners assisted by training and screening procedure. Patient Education and Counseling, 63, 232-238.

UNITED STATES PUBLIC HEALTH SERVICE (1964) Smoking and Health: Report of the Advisory Committee to the Surgeon General of the Public Health Service.

USDHHS (2004) The health consequences of smoking: a report of the Surgeon General, Washington, D.C., Department of Health and Human Services, National Center for Chronic Disease Prevention and Health Promotion, Office on Smoking and Health.

VALLERAND, R. J. (1997) Towards a hierarchical model of intrinsic and extrinsic motivation. IN ZANNA, M. P. (Ed.) Advances in experimental social psychology. New York, NY, Academic Press.

VAN DEN BERG, M., DE BAKKER, D., KOLTHOF, E., CARDOL, M. \& VAN DEN BRINK-MUINEN, A. (2003) De werkdruk van de huisarts: zorgvraag en arbeidsduur in 1987 en in 2001. Medisch Contact, 58, 1054-1056.

VAN DEN BRUEL, A., CLEEMPUT, I., AN LINDEN, A., SCHOEFS, D., RAMAEKERS, D. \& BONNEUX, L. (2004) Effectiveness and Cost-Effectiveness of Smoking Cessation Strategies, Brussel, Belgian Health Care Knowledge Center.

VAN DER WEIDE, M. \& SMITS, J. (2004) Adoption of innovations by specialised nurses: personal, work and organisational characteristics. Health Policy, 68, 81-92.

VAN DIJK, J. A. G. M. (2003) De digitale kloof wordt dieper: van ongelijkheid in bezig naar ongelijkheid in vaardigheden en gebruik van ICT, Zaandam, The Netherlands, Kwak \& van Daalen \& Ronday.

VAN GELDER, B. M., POOS, M. J. J. C. \& ZANTINGE, E. M. (2011) Wat zijn de mogelijke gezondheidsgevolgen van roken? Volksgezondheid Toekomst Verkenning, Nationaal Kompas Volksgezondheid. Bilthoven, RIVM. 
VAN KEULEN, H. M., BOSMANS, J. E., VAN TULDER, M. W., SEVERENS, J. L., DE VRIES, H., BRUG, J. \& MESTERS, I. (2010) Cost-effectiveness of tailored print communication, telephone motivational interviewing, and a combination of the two: results of an economic evaluation alongside the Vitalum randomized controlled trial. International Journal of Behavioural Nutrion and Physcial Activity, 7, 64.

VAN KEULEN, H. M., MESTERS, I., BRUG, J., AUSEMS, M., CAMPBELL, M., RESNICOW, K., ZWIETERING, P. J., VAN BREUKELEN, G., VAN MECHELEN, W., SEVERENS, J. L. \& DE VRIES, H. (2008) Vitalum study design: RCT evaluating the efficacy of tailored print communication and telephone motivational interviewing on multiple health behaviors. BMC Public Health, 8, 216.

VAN OSCH, L., LECHNER, L., REUBSAET, A. \& DE VRIES, H. (2010) From theory to practice: An explorative study into the instrumentality and specificity of implementation intentions. Psychology \& Health, 25, 351-64.

VAN OSCH, L., LECHNER, L., REUBSAET, A., WIGGER, S. \& DE VRIES, H. (2008) Relapse prevention in a national smoking cessation contest: Effects of coping planning. British Journal of Health Psychology, 13, 525-35.

VAN SON, L., VRIJHOEF, H. J., CREBOLDER, H. F., VAN HOEF, L. \& GEUSMANS, G. (2004) De huisarts onderteunt. Een RCT naar het effect van praktijkondersteuner bij astma, COPD en diabetes. Huisarts \& Wetenschap, 47, 15-21.

VAN STRALEN, M. M., DE VRIES, H., MUDDE, A. N., BOLMAN, C. \& LECHNER, L. (2010) The long-term efficacy of two computer-tailored physical activity interventions for older adults: main effects and mediators. Health Psychology, 30, 442-52.

VAN STRALEN, M. M., KOK, G., DE VRIES, H., MUDDE, A. N., BOLMAN, C. \& LECHNER, L. (2008) The Active plus protocol: systematic development of two theory- and evidence-based tailored physical activity interventions for the over-fifties. BMC Public Health, 8, 399.

VAN WEEL, C., COEBERGH, J. W., DRENTHEN, T., SCHIPPERS, G. M., VAN SPIEGEL, P. I., ANDERSON, P. D., VAN BLADEREN, F. A. \& VAN VEENENDAAL, H. (2005) Richtlijn 'Behandeling van tabaksverslaving'. Nederlands Tijdschrift voor Geneeskunde, 149, 17-21.

VAN WEEL, C., SCHERS, H. \& TIMMERMANS, A. (2012) Health care in the Netherlands. Journal of the American Board of Family Medicine, 25 Suppl 1, S12-7.

VANGELI, E., STAPLETON, J., SMIT, E. S., BORLAND, R. \& WEST, R. (2011) Predictors of attempts to stop smoking and their success in adult general population samples: A systematic review. Addiction, 106, 2110-2121.

VELICER, W. F., HUGHES, S. L., FAVA, J. L., PROCHASKA, J. O. \& DICLEMENTE, C. C. (1995) An empirical typology of subjects within stage of change. Addictive Behaviors, 20, 299-320.

VELICER, W. F., NORMAN, G. J., FAVA, J. L. \& PROCHASKA, J. O. (1999) Testing 40 predictions from the transtheoretical model. Addictive Behaviors, 24, 455-469.

VELICER, W. F. \& PROCHASKA, J. O. (1999) An expert system intervention for smoking cessation. Patient Education and Counseling, 36, 119-29.

VELICER, W. F., PROCHASKA, J. O., ROSSI, J. S. \& SNOW, M. G. (1992) Assessing outcome in smoking cessation studies. Psychology Bulletin, 111, 23-41.

VEMER, P., RUTTEN VAN MOLKEN, M. P., KAPER, J., HOOGENVEEN, R. T., VAN SCHAYCK, C. P. \& FEENSTRA, T. L. (2010) If you try to stop smoking, should we pay for it? The cost-utility of reimbursing smoking cessation support in the Netherlands. Addiction, 105, 1088-97.

VERDONK-KLEINJAN, W. M., CANDEL, M. J., KNIBBE, R. A., WILLEMSEN, M. C. \& DE VRIES, H. (2011) Effects of a workplace-smoking ban in combination with tax increases on smoking in the Dutch population. Nicotine and Tobacco Research, 13, 412-8.

VITORIA, P. D., SALGUEIRO, M. F., SILVA, S. A. \& DE VRIES, H. (2009) The impact of social influence on adolescent intention to smoke: combining types and referents of influence. British Journal of Health Psychology, 14, 681-99. 
VOGT, F., HALL, S. \& MARTEAU, T. M. (2005) General practitioners' and family physicians' negative beliefs and attitudes towards discussing smoking cessation with patients: a systematic review. Addiction, 100, 1423-31.

WANGBERG, S. C., BERGMO, T. S. \& JOHNSEN, J. A. (2008) Adherence in Internet-based interventions. Patient Preference and Adherence, 2, 57-65.

WANGBERG, S. C., NILSEN, O., ANTYPAS, K. \& GRAM, I. T. (2011) Effect of tailoring in an internetbased intervention for smoking cessation: randomized controlled trial. Journal of Medical Internet Research, 13, e121.

WARMERDAM, L., SMIT, F., VAN STRATEN, A., RIPER, H. \& CUIJPERS, P. (2010) Cost-utility and costeffectiveness of internet-based treatment for adults with depressive symptoms: randomized trial. Journal of Medical Internet Research, 12, e53.

WEBB, T. L. (2009) Commentary on Shahab \& McEwen (2009): Understanding and preventing attrition in online smoking cessation interventions: a self-regulatory perspective. Addiction, 104, 1805-6.

WEBB, T. L., JOSEPH, J., YARDLEY, L. \& MICHIE, S. (2010) Using the internet to promote health behavior change: a systematic review and meta-analysis of the impact of theoretical basis, use of behavior change techniques, and mode of delivery on efficacy. Journal of Medical Internet Research, 12, e4.

WEBBER, K. H., TATE, D. F. \& QUINTILIANI, L. M. (2008) Motivational interviewing in internet groups: a pilot study for weight loss. Journal of the American Dietician Associations, 108, 1029-32.

WEINSTEIN, N. D., ROTHMAN, A. J. \& SUTTON, S. R. (1998) Stage theories of health behavior: conceptual and methodological issues. Health Psychology, 17, 290-299.

WEINSTEIN, N. D. \& SANDMAN, P. M. (2002) The Precaution Adoption Process Model. IN GLANZ, K. (Ed.) Health Behavior and Health Education: Theory, Research and Practice. San Francisco, Jossey-Bass.

WEST, R. (2005) Time for a change: putting the Transtheoretical (Stages of Change) Model to rest. Addiction, 100, 1036-9.

WEST, R. Feasibility of a national longitudinal study ('The Smoking Toolkit Study') to monitor smoking cessation and attempts at harm reduction in the UK. Accessed: 2010 October 1; http://www.smokinginengland.info/file.asp?file=Ref/stp001.pdf\&refid=19 Archived by WebCite at: http://www.webcitation.org/5mcvjDQai

WEST, R. (2009) The multiple facets of cigarette addiction and what they mean for encouraging and helping smokers to stop. COPD, 6, 277-83.

WEST, R., HAJEK, P., STEAD, L. \& STAPLETON, J. (2005) Outcome criteria in smoking cessation trials: proposal for a common standard. Addiction, 100, 299-303.

WEST, R. \& HARDY, A. (2006) Theory of addiction, Oxford, Blackwell.

WEST, R., MCEWEN, A., BOLLING, K. \& OWEN, L. (2001) Smoking cessation and smoking patterns in the general population: a 1-year follow-up. Addiction, 96, 891-902.

WEST, R. \& SOHAL, T. (2006) "Catastrophic" pathways to smoking cessation: findings from national survey. British Medical Journal, 332, 458-60.

WETTA-HALL, R., BERRY, M. \& GOO, M. J. (2005) Factors influencing nurses' smoking cessation assessment and counselling practice. Journal of Addictions Nursing, 16, 131-135.

WHO (1986) Ottawa Charter for Health Promotion. Geneva, World Health Organization.

WHO (2008) WHO Report on the Global Tobacco Epidemic, 2008: the MPOWER package. Geneva, World Health Organization.

WHYTE, R. E., WATSON, H. E. \& MCINTOSH, J. (2006) Nurses' opportunistic interventions with patients in relation to smoking. Journal of Advanced Nursing, 55, 568-577.

WILLEMSEN, M. C., DE VRIES, H., VAN BREUKELEN, G. \& GENDERS, R. (1998) Long-term effectiveness of two Dutch work site smoking cessation programs. Health Education and Behaviour, 25, 418-35. 
WILLIAMS, G. C., GAGNE, M., RYAN, R. M. \& DECI, E. L. (2002) Facilitating autonomous motivation for smoking cessation. Health Psychology, 21, 40-50.

WILLIAMS, G. C., MCGREGOR, H., SHARP, D., KOUIDES, R. W., LEVESQUE, C. S., RYAN, R. M. \& DECI, E. L. (2006a) A Self-Determination Multiple Risk Intervention Trial to Improve Smokers' Health. Journal of General Internal Medicine.

WILLIAMS, G. C., MCGREGOR, H. A., SHARP, D., LEVESQUE, C., KOUIDES, R. W., RYAN, R. M. \& DECI, E. L. (2006b) Testing a self-determination theory intervention for motivating tobacco cessation: supporting autonomy and competence in a clinical trial. Health Psychology, 25, 91101.

WILLIAMS, G. C., PATRICK, H., NIEMIEC, C. P., RYAN, R. M., DECI, E. L. \& LAVIGNE, H. M. (2011) The smoker's health project: a self-determination theory intervention to facilitate maintenance of tobacco abstinence. Contemporary Clinical Trials, 32, 535-43.

WYNDER, E. L., BROSS, I. J. \& DAY, E. (1956) A study of environmental factors in cancer of the larynx. Cancer, 9, 86-110.

YARNALL, K., POLLAK, K., KRAUSE, K. \& MICHENER, J. (2003) Primary care: Is there enough time for prevention? American Journal of Public Health, 93, 635-641.

ZHOU, X., NONNEMAKER, J., SHERRILL, B., GILSENAN, A. W., COSTE, F. \& WEST, R. (2009) Attempts to quit smoking and relapse: factors associated with success or failure from the ATTEMPT cohort study. Addictive Behaviors, 34, 365-73.

ZWAR, N. A., RICHMOND, R. L., FORLONGE, G. \& HASAN, I. (2010) Feasibility and effectiveness of nurse-delivered smoking cessation counselling combined with nicotine replacement in Australian general practice. Drug and Alcohol Review, 30, 583-8. 


\section{APPENDIX 1}

\section{A tailored smoking cessation advice for a respondent who reported to still be smoking at six- month follow-up and whose self-efficacy to quit has decreased since baseline.}

Step 3. Have confidence in the success of your quit attempt!

You are now less confident that you will succeed in not smoking than the before. That's a pity, because when you feel more confident that you will succeed, it will become easier for you to quit smoking. Therefore, we will go through those situations again, in which you indicated it will be hard for you not to smoke. And on the next pages, we will once more give you the opportunity to make specific plans to cope with these difficult situations. By making specific plans on how to ensure you will not smoke in these situations, the chances will increase that you will succeed in becoming a non-smoker.

- You are not sure whether you will succeed not to smoke when someone offers you a cigarette. And you are right, it is indeed very hard to say 'no' in such a situation. Perhaps you can re-read the advice we have given you last time. In this advice, we extensively described how you can learn to say 'no' in such situations. You will find your previous feedback letter by clicking on the ' $m y$ advice' button in the left menu.

- You indicate that you do not think you will manage not to smoke when you're taking a break. For many smokers, is has become a habit to smoke while taking a break. But why don't you try not to stick around your smoking colleagues, when you often smoke during breaks at work? There are probably many other people who do not smoke and with whom you could have a nice chat. 



\section{APPENDIX 2}

\section{Results from complete-case analyses}

Table 5.5 Effects of the web-based smoking cessation intervention on several behavioural outcomes at six-week follow-up among Dutch adult smokers ( $\mathrm{N}=449)$ recruited from December 2009 up until June 2010 - complete cases

\begin{tabular}{|c|c|c|c|c|c|c|c|c|c|}
\hline & \multicolumn{3}{|c|}{ 24-hour ppaa } & \multicolumn{3}{|c|}{ 7-day ppa ${ }^{a}$} & \multicolumn{3}{|c|}{$\begin{array}{l}\text { Prolonged } \\
\text { abstinence }\end{array}$} \\
\hline & $\mathrm{OR}^{\mathrm{b}}$ & $95 \% \mathrm{CI}^{\mathrm{C}}$ & $\mathrm{P}$ & $\mathrm{OR}^{\mathrm{b}}$ & $95 \% \mathrm{CI}^{\mathrm{C}}$ & $\mathrm{P}$ & $\mathrm{OR}^{\mathrm{b}}$ & $95 \% \mathrm{CI}^{\mathrm{C}}$ & $\mathrm{P}$ \\
\hline Intervention ${ }^{\mathrm{d}}$ & 2.12 & $1.41-3.17$ & .000 & 2.43 & $1.55-3.79$ & .000 & 2.13 & $1.33-3.42$ & .002 \\
\hline Interventiond & 2.07 & $1.38-3.13$ & .001 & 2.50 & $1.59-3.94$ & .000 & 2.14 & $1.32-3.46$ & .002 \\
\hline Medium educatione & .63 & $.36-1.09$ & .10 & .57 & $.32-1.02$ & .06 & .59 & $.31-1.09$ & .09 \\
\hline High educatione & 1.00 & $.57-1.76$ & 1.00 & .68 & $.37-1.24$ & .21 & .80 & $.43-1.51$ & .49 \\
\hline
\end{tabular}

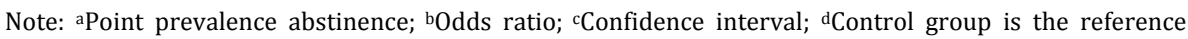
category; Low education is the reference category.

Table 5.6 Effects of the web-based smoking cessation intervention on several behavioural outcomes at six-month follow-up among Dutch adult smokers ( $\mathrm{N}=291)$ recruited from December 2009 up until June 2010 - complete cases

\begin{tabular}{lccccccccc}
\hline & \multicolumn{3}{c}{ 24-hour ppa } & & & 7-day ppa & & \multicolumn{3}{c}{$\begin{array}{c}\text { Prolonged } \\
\text { abstinence }\end{array}$} \\
& $\mathrm{OR}^{\mathrm{b}}$ & $95 \% \mathrm{CI}^{\mathrm{c}}$ & $\mathrm{P}$ & $\mathrm{OR}^{\mathrm{b}}$ & $95 \% \mathrm{CI}^{\mathrm{c}}$ & $\mathrm{P}$ & $\mathrm{OR}^{\mathrm{b}}$ & $95 \% \mathrm{CI}^{\mathrm{c}}$ & $\mathrm{P}$ \\
\hline Intervention $^{\mathrm{d}}$ & 1.69 & $1.02-2.81$ & .04 & 1.51 & $.90-2.54$ & .12 & 1.29 & $.67-2.49$ & .45 \\
Intervention $^{\mathrm{d}}$ & 1.65 & $.98-2.76$ & .06 & 1.54 & $.90-2.61$ & .11 & 1.46 & $.74-2.87$ & .27 \\
Medium education $^{\mathrm{e}}$ & .68 & $.33-1.37$ & .28 & .65 & $.32-1.32$ & .24 & .45 & $.20-1.01$ & .05 \\
High education $^{\mathrm{e}}$ & 1.02 & $.50-2.08$ & .97 & .76 & $.37-1.57$ & .45 & .37 & $.15-.89$ & .03 \\
\hline
\end{tabular}

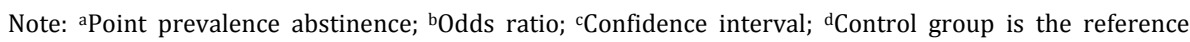
category; elow education is the reference category. 



\section{SUMMARY}

Despite the fact that each year 5.4 million people die from the consequences of smoking, more than one billion people in the world continue to smoke. To reduce both the number of people suffering from smoking-related illnesses and the number of smoking-related deaths, continued efforts are required to move smokers towards cessation. As the effects of stand-alone interventions have been found to typically be limited, the smoking cessation intervention PAS (Personal Advice in Stopping smoking) combines two previously developed effective micro-level interventions: computer-tailoring and behavioural counselling by a practice nurse working in primary care. The first aim of the present dissertation was to develop and to evaluate PAS with regard to its effectiveness, cost-effectiveness and feasibility (chapters 2 up to and including 8). The second aim of this dissertation was to inform the development of future smoking cessation interventions by contributing to an increased understanding of smokers' motivation to quit, as a positive motivation is a necessary though not always sufficient prerequisite for smoking cessation to occur (chapters 9 and 10).

Chapter 1 provides a general introduction to the studies presented in this dissertation. It presents the background of the studies conducted as well as the specific research questions this dissertation aims to answer. In addition, this chapter describes the theoretical framework used for most of the studies conducted, i.e. the I-Change Model.

Chapter 2 provides an extensive description of the development of PAS, consisting of an Internet-based multiple computer-tailored smoking cessation programme and tailored counselling by a practice nurse.

Chapter 3 describes a cross-sectional study among Dutch practice nurses and nurse practitioners $(\mathrm{N}=139)$ that aimed to identify factors associated with their intention to adopt the new smoking cessation intervention PAS. Both a positive attitude and a positive social environment were positively associated with the intention to adopt PAS, while practice nurses' and nurse practitioners' satisfaction with current smoking cessation activities appeared to be negatively correlated with the intention to adopt.

Chapter 4 describes a study investigating the influence of recruitment strategy on the reach and (short-term) effect of the Internet-based multiple tailored smoking cessation programme. Recruitment via general practices $(\mathrm{N}=409)$ yielded fewer respondents than recruitment via the mass media $(\mathrm{N}=1154)$. However, respondents recruited via general practices were more likely to quit smoking: they were significantly less addicted to nicotine, more motivated to quit smoking as well as to maintain non-smoking and they were more likely to complete the intervention. This 
suggests that the choice for a particular recruitment strategy may determine the number and type of smokers recruited and might consequently influence the intervention's potential public health impact.

Chapter 5 describes a randomized controlled trial (RCT) investigating the effectiveness of the Internet-based multiple computer-tailored smoking cessation programme among a general population sample of Dutch adult smokers $(\mathrm{N}=1123)$. Positive intervention effects on smoking abstinence rates were identified for the entire study population. Whereas these effects could be detected significantly after six weeks, however, they could no longer be identified after six months.

Chapter 6 presents an RCT testing the effectiveness of the Internet-based multiple computer-tailored smoking cessation programme with and without counselling by a practice nurse among Dutch adult smokers recruited via their general practice $(\mathrm{N}=414)$. Both interventions were compared with usual care in the general practice setting and with each other. The Internet-based multiple computer-tailored smoking cessation programme appeared to be more effective than usual care in increasing abstinence rates among three subgroups of smokers: smokers with low levels of addiction, smokers who made many previous quit attempts and older smokers. No intervention effects on smoking abstinence were identified when one tailored feedback letter was replaced by a tailored counselling session by a practice nurse.

Chapter 7 describes the trial-based economic evaluation that was conducted to investigate the cost-effectiveness and cost-utility of both PAS interventions when implemented in the general practice setting. This economic evaluation study extended recommendations from the studies presented in chapters 5 and 6 concerning the Internet-based multiple computer-tailored smoking cessation programme; the cost-effectiveness analysis, using prolonged abstinence as the outcome measure, showed that this intervention had the highest probability of being costeffective. Compared with usual care, in this intervention group $€ 5,100$ had to be paid for each additional respondent being abstinent. Yet, interpretation of these results is difficult as no information exists on the amount of money that society is willing to pay per abstinent participant. With regard to cost-utility analyses, using quality of life as the outcome measure, usual care was most probably most efficient.

Chapter 8 describes a cross-sectional study that investigated the determinants of practice nurses' $(\mathrm{N}=56)$ intention to implement PAS after a trial period. Both attitude and innovation characteristics (i.e. relative advantage, compatibility, complexity, trialability and observability) were independently, positively associated with practice nurses' intention to implement. In addition, involvement in the study, defined in terms of recruitment success, and perceived patient support showed a significant positive association with intention.

Chapter 9 describes the results of a longitudinal study which aimed to assess the predictive value of three different aspects of motivation, i.e. desire, duty and intention to quit, in predicting attempts to quit smoking among a nationally representative sample of British smokers $(\mathrm{N}=5593)$. It was revealed that although duty 
(the belief that one ought to quit) was the most commonly reported motive for quitting smoking, only a desire and intention to quit smoking were significant independent predictors of quit attempts.

Chapter 10 presents a study that aimed to investigate whether subtypes exist among smokers in the contemplation stage of change $(\mathrm{N}=194)$. Three clusters of smokers could be identified: Early, Progressing and Disengaged contemplators. Interestingly, no subtype was identified representing the Classic contemplator, scoring high on both pros and cons of quitting and low on self-efficacy.

In chapter 11 of this dissertation, the general discussion, the results presented in chapters 2 to 10 are integrated and discussed, resulting in recommendations for theory, future research and practice. It can be concluded 1) that before nationwide implementation, PAS should first be further improved; 2) that future research should aim at identifying an acceptable cut-off point for the willingness to pay per abstinent participant; 3) that the choice for a particular recruitment strategy should be informed by an intervention's objectives as it is expected to influence the reach and subsequent potential public health impact of an intervention; 4) that to disseminate PAS and similar interventions, it is especially important to create a positive attitude and a positive social environment and to help practice nurses in the recruitment of their smoking patients; 5) that interventions aimed at promoting smoking cessation behaviour need to go beyond creating the belief that smokers ought to quit smoking by making them want to quit and/or intend to quit smoking; and 6) that for smokers categorized as contemplators item-based tailoring might be preferable over stage-based tailoring. Lastly, this chapter describes methodological and practice considerations in light of which the results presented in this dissertation should be interpreted. 



\section{SAMENVATTING}

Ondanks het feit dat jaarlijks 5.4 miljoen mensen overlijden aan de gevolgen van roken, rookt nog altijd meer dan één biljoen mensen wereldwijd. Om het aantal mensen dat lijdt aan rokengerelateerde ziekten en om het aantal rokengerelateerde doden te verminderen, zijn niet aflatende inspanningen nodig om rokers ertoe te bewegen te stoppen. Aangezien de effecten van op zichzelf staande interventies doorgaans beperkt zijn, combineert de stoppen-met-roken interventie PAS (Persoonlijk Advies bij het Stoppen met roken) twee eerder ontwikkelde effectieve micro-level interventies: advies-op-maat en gedragsmatige counselling door een praktijkondersteuner. Het eerste doel van dit proefschrift was om PAS te ontwikkelen en te evalueren wat betreft effectiviteit, kosteneffectiviteit en haalbaarheid (hoofdstuk 2 tot en met 8 ). Het tweede doel van dit proefschrift was, aangezien een positieve motivatie een noodzakelijke maar niet altijd voldoende voorwaarde is voor stoppen met roken, om bij te dragen aan een beter inzicht in rokers' motivatie om te stoppen en zo informatie te verschaffen voor de ontwikkeling van toekomstige stoppen-met-roken interventies (hoofdstukken 9 en 10).

Hoofdstuk 1 geeft een algemene introductie van de studies die in dit proefschrift worden beschreven. Het presenteert de achtergrond van de studies die zijn uitgevoerd als ook de specifieke onderzoeksvragen die dit proefschrift beoogt te beantwoorden. Verder beschrijft dit hoofdstuk het theoretisch kader dat is gebruikt voor de meeste studies, d.w.z. het I-Change Model.

Hoofdstuk 2 geeft een uitgebreide beschrijving van de ontwikkeling van PAS, bestaande uit een meervoudig advies-op-maat programma aangeboden via het internet en persoonlijke counselling door een praktijkondersteuner.

Hoofdstuk 3 beschrijft een cross-sectionele studie onder Nederlandse praktijkondersteuners en praktijkverpleegkundigen $(\mathrm{N}=139)$ die beoogde factoren te identificeren die geassocieerd zijn met de intentie op de nieuwe stoppen-met-roken interventie PAS te gebruiken. Zowel een positieve attitude als een positieve sociale omgeving bleken positief geassocieerd te zijn met de intentie om PAS te gebruiken. De tevredenheid van praktijkondersteuners en praktijkverpleegkundigen met hun huidige stoppen-met-roken activiteiten bleek daarentegen negatief geassocieerd te zijn met de intentie om PAS te gebruiken.

Hoofdstuk 4 beschrijft een studie die de invloed onderzocht van twee verschillende wervingsstrategieën op het bereik en (korte termijn) effect van het meervoudig advies-op-maat programma aangeboden via het internet. Werving via huisartsenpraktijken $(\mathrm{N}=409)$ resulteerde in minder deelnemers dan werving via de massamedia $(\mathrm{N}=1154)$. Respondenten die werden geworven via huisartsenpraktijken hadden echter een grotere kans om te stoppen met roken: zij waren significant 
minder verslaafd aan nicotine, meer gemotiveerd om te stoppen met roken en om het vol te houden om niet te roken en zij waren meer geneigd de interventie af te maken. Dit suggereert dat de keuze voor een bepaalde wervingsstrategie van invloed kan zijn op het aantal en het type rokers dat geworven wordt voor een interventie zoals PAS. Als gevolg hiervan kan deze keuze van invloed zijn op de potentiële impact van deze interventie op de volksgezondheid.

Hoofdstuk 5 beschrijft een gerandomiseerd gecontroleerd experiment (RCT) waarin de effectiviteit werd onderzocht van het meervoudig advies-op-maat programma aangeboden via het internet binnen een steekproef van volwassen rokers uit de algemene Nederlandse bevolking ( $\mathrm{N}=1123)$. Positieve interventie effecten op abstinentie werden gevonden voor de gehele studiepopulatie. Terwijl deze positieve effecten significant konden worden aangetoond na zes weken, waren ze na zes maanden echter niet meer te ontdekken.

Hoofdstuk 6 presenteert een RCT die de effectiviteit onderzocht van het meervoudig advies-op-maat programma aangeboden via het internet met en zonder persoonlijke counselling door een praktijkondersteuner onder Nederlandse volwassen rokers geworven via hun huisartsenpraktijk $(\mathrm{N}=414)$. Beide interventies werden vergeleken met de gebruikelijke zorg in de huisartsenpraktijk en met elkaar. Het meervoudig advies-op-maat programma aangeboden via het internet bleek effectiever dan de gebruikelijke zorg in het doen toenemen van abstinentie aantallen voor drie subgroepen van rokers: rokers met lage verslavingsscores, rokers die al veel eerdere stoppogingen hadden gedaan en oudere rokers. Geen interventie effecten werden gevonden van het meervoudig advies-op-maat programma aangeboden via het internet gecombineerd met persoonlijke counselling door een praktijkondersteuner.

Hoofdstuk 7 beschrijft de economische evaluatie studie die is gedaan om de kosteneffectiviteit en kostenutiliteit van beide PAS interventies te onderzoeken wanneer deze werden geïmplementeerd in de huisartsenpraktijk. Deze economische evaluatie studie bevestigde de aanbevelingen die werden gedaan in de studies gepresenteerd in de hoofdstukken 5 en 6 wat betreft het meervoudig advies-opmaat programma aangeboden via het internet; de kosteneffectiviteitanalyse, waarbij geprolongeerde abstinentie als uitkomstmaat werd gebruikt, liet zien dat deze interventie de grootste kans had om kosteneffectief te zijn. In vergelijking met de gebruikelijke zorg moest binnen deze interventiegroep $€ 5,100$ betaald worden voor iedere extra abstinente deelnemer. De interpretatie van deze resultaten is echter moeilijk aangezien geen informatie beschikbaar is over het bedrag dat de maatschappij bereid is te betalen voor een extra abstinente deelnemer. Wat betreft kostenutiliteit, waarbij kwaliteit van leven als uitkomstmaat werd gebruikt, was de gebruikelijke zorg waarschijnlijk het meest efficiënt.

Hoofdstuk 8 beschrijft een cross-sectionele studie die tot doel had om de determinanten te onderzoeken van de intentie van praktijkondersteuners $(\mathrm{N}=56)$ om PAS te implementeren na een proefperiode. Zowel attitude als innovatie karakteristieken (d.w.z. relatief voordeel, compatibiliteit, complexiteit, uitprobeerbaarheid en 
waarneembaarheid) waren onafhankelijk van elkaar positief geassocieerd met de intentie van praktijkondersteuners om PAS te implementeren. Verder lieten betrokkenheid in de studie, gedefinieerd in termen van wervingssucces, en waargenomen steun van patiënten een significante positieve associatie zien met de intentie om PAS te implementeren.

Hoofdstuk 9 beschrijft de resultaten van een longitudinale studie die tot doel had de voorspellende waarde van drie verschillende aspecten van motivatie, d.w.z. wens, plicht en intentie om te stoppen, te onderzoeken wat betreft het voorspellen van stoppogingen binnen een steekproef van rokers representatief voor de algemene Britse bevolking ( $\mathrm{N}=5593)$. We vonden dat ondanks dat plicht (de gedachte dat men zou moeten stoppen) het meeste gerapporteerde motief was om te stoppen met roken, alleen de wens en de intentie om te stoppen met roken significante onafhankelijke voorspellers waren van stoppogingen.

Hoofdstuk 10 presenteert een studie die tot doel had te onderzoeken of er subtypes bestaan binnen rokers in de contemplatie fase van gedragsverandering $(\mathrm{N}=194)$. Drie clusters van rokers konden worden geïdentificeerd: Early, Progressing en Disengaged contemplatoren. Interessant genoeg kon er geen subtype geïdentificeerd worden dat de Classic contemplator vertegenwoordigde en hoog scoorde wat betreft de voordelen en nadelen van stoppen en laag wat betreft eigeneffectiviteit.

In hoofdstuk 11 van dit proefschrift, de algemene discussie, worden de resultaten die zijn gepresenteerd in de hoofdstukken 2 tot en met 10 geïntegreerd en bediscussieerd, resulterend in aanbevelingen voor theorie, toekomstig onderzoek en praktijk. Het kan worden geconcludeerd 1) dat voordat wordt overgegaan tot landelijke implementatie, PAS eerst verbeterd dient te worden; 2) dat toekomstig onderzoek zich moet richten op het identificeren van een acceptabel bedrag dat de maatschappij bereid is te betalen per abstinente deelnemer; 3) dat de keuze voor een bepaalde wervingsstrategie moet worden bepaald aan de hand van interventiedoelen, omdat deze keuze van invloed kan zijn op het bereik van de interventie en de resulterende impact van de interventie op de volksgezondheid; 4) dat voor de disseminatie van PAS en vergelijkbare interventies het vooral belangrijk is om een positieve attitude en een positieve sociale omgeving te creëren, als ook om praktijkondersteuners te helpen bij de werving van hun rokende patiënten; 5) dat stoppen-met-roken interventies verder moeten gaan dat het creëren van de gedachte dat rokers zouden moeten stoppen met roken en rokers' wens en/of intentie om te stoppen met roken moeten vergroten; 6) dat voor rokers die worden gecategoriseerd als contemplatoren item-gebaseerd advies-op-maat wellicht verkozen dient te worden boven fase-gebaseerd advies-op-maat. Tenslotte beschrijft dit hoofdstuk methodologische en praktische overwegingen in het licht waarvan de resultaten gepresenteerd in dit proefschrift moeten worden geïnterpreteerd. 



\section{DANKWOORD}

Graag wil ik hier een aantal mensen bedanken die op de een of andere manier hebben bijgedragen aan de totstandkoming van dit proefschrift.

Allereerst wil ik de deelnemende praktijkondersteuners en rokers bedanken, want zonder hen had ik mijn onderzoek niet uit kunnen voeren. De praktijkondersteuners wil ik bedanken voor hun enthousiasme en inzet. De rokers voor het achterlaten van hun veelal leuke opmerkingen aan het einde van iedere vragenlijst. Beiden hebben ertoe bijgedragen dat ik ook de praktische kant van het onderzoek als zeer positief heb ervaren.

Een speciaal woord van dank voor mijn promotieteam. Hein, bedankt voor de kans die je me hebt gegeven om onder jouw supervisie te promoveren. De afgelopen vier jaar zijn we het zeker niet altijd met elkaar eens geweest, maar onze discussies hebben er uiteindelijk voor gezorgd dat ik dit proefschrift naar een hoger niveau heb weten te tillen. Ciska, een betere dagelijks begeleidster had ik me niet kunnen wensen. Terwijl je hebt me gestimuleerd om al het mogelijke uit dit promotietraject te halen, bleef je altijd down-to-earth en realistisch; mijn inziens een goede combinatie.

Dank ook aan de leescommissie en corona voor het beoordelen van mijn proefschrift en het stellen van de (hopelijk niet al te moeilijke) vragen tijdens mijn verdediging.

Ik wil alle leden van het projectteam bedanken voor hun advies gedurende de duur van het project; een aantal mensen in het bijzonder. Silvia, ik vond het een enorm prettig om met je samen te werken tijdens het doen van de kosteneffectiviteitstudie, iets wat ik me tijdens elk overleg weer realiseerde. Bedankt, Math, voor je hulp met het analyseren van de effecten van PAS. Vincent, bedankt voor je inzet als onderzoeksassistent; jij maakte de autoritjes naar praktijkondersteuners in alle uithoeken van het land een stuk gezelliger!

Special thanks to Robert, Martin, Falko, Ed and Geof. I have very much enjoyed working at each of your departments. As one of the propositions says: 'Variety is the spice of life'. Working with you has been very inspiring and has further increased my motivation to continue my scientific career.

Bedankt, alle collega's van de vakgroep Gezondheidsbevordering. Voor de gezelligheid, de borrels, als ook voor de inhoudelijke discussies. In het bijzonder natuurlijk 
de Goulash; Francine, Judith, Eva, Karen, Michelle en Leonieke. Jullie zorgden (en zorgen) ervoor dat het elke dag weer een feestje is om naar het werk te gaan! Francine, onze gezamenlijke liefde voor Milow, flauwe grappen en lekker eten, maar ook onze gezamenlijke frustraties op weg naar PhD-island maakten (en maken) je de perfecte roomie en ik ben blij dat jij mijn paranimf wilt zijn. Patricia, Marja en Leon, jullie bedankt voor alle hulp bij de logistieke, financiële en computer gerelateerde aspecten van mijn project.

Ik wil ook graag alle leden van de Journal Club Maastricht bedanken voor de gezellige etentjes en levendige discussies, wetenschappelijk maar ook minder wetenschappelijk. $\mathrm{Nu}$ mijn proefschrift af kan ik me weer toeleggen op het organiseren van een volgende activiteit :)

In het bijzonder wil ik mijn ouders en mijn zusje bedanken. Pap en mam, dankjewel voor jullie steun tijdens mijn promotietraject en daarbuiten; het is fijn om te weten dat ik altijd bij jullie terecht kan. Marlou, bedankt voor het duwtje in de rug dat ik nodig had om te kiezen voor promoveren in Maastricht ('vier jaar is toch zó voorbij?!'); ik heb geen seconde spijt gehad van mijn beslissing. Fijn dat jij mijn paranimf wilt zijn!

En tenslotte bedankt, Wilko. Voor je luisterend oor en je relativeringsvermogen, maar eigenlijk voor alles. Je bent de leukste! 


\section{CURRICULUM VITAE}

Eline Suzanne Smit was born in Delft, the Netherlands, on the $5^{\text {th }}$ of January 1985. After receiving her Gymnasium diploma from the St. Stanislas College in Delft, she moved to Maastricht where she studied Health Sciences at Maastricht University. As a part of this bachelor's curriculum, she followed several elective courses at the University of Kuopio, Finland. She obtained her degree in 2006, after which she started a master's programme in Health Education and Health Promotion at Maastricht University. While studying for her master's degree, which she obtained in 2007, she worked as a student assistant at the Department of Health Promotion of this same university.

In 2008, Eline started working as a PhD-student on the project described in this dissertation. In 2010, she obtained a CAPHRI travel grant to work in the United Kingdom for a period of three months, during which she gained research experience working at three different universities. Up until now, Eline has presented her research at different national and international conferences. For her poster presentation at the European Conference on Tobacco or Health, held in Amsterdam in 2011, she obtained the award for the best poster 'Elevator Pitch'.

The author is currently working as a postdoctoral researcher at the Department of Health Promotion, a position for which she obtained a two-year CAPHRI 'Center of Excellence' postdoc grant. To obtain input for her postdoctoral research, she recently received a travel grant from the Dutch Cancer Society that she used to visit the Motivation Research Group at the University of Rochester (Rochester, NY, USA). 



\section{PUBLICATION LIST}

d.d. July 14,2012

\section{Publications from this thesis}

Smit, E.S., Hoving, C., de Vries, H. (2010). Does a Typical Contemplator Exist? Three clusters of smokers in contemplation, Health Education Research, 25(1), 61-73.

Smit, E.S., de Vries, H., Hoving, C. (2010). The PAS study: A Randomized Controlled Trial evaluating the effectiveness of a web-based multiple tailored smoking cessation programme and tailored counselling by practice nurses, Contemporary Clinical Trials, 31, 251-258.

Smit, E.S., Fidler, J.A., West, R. (2010). The role of desire, duty and intention in predicting attempts to quit smoking, Addiction, 106(4), 844-851.

Smit, E.S., Hoving, C., Cox, V.C.M., de Vries, H. (2011). Influence of recruitment strategy on the reach and effect of a web-based multiple tailored smoking cessation intervention amongst Dutch adult smokers, Health Education Research, 27(2), 191199.

Smit, E.S., de Vries, H., Hoving, C. (2012). The effects of a web-based multiple tailored smoking cessation programme: a randomized controlled trial, Journal of Medical Internet Research, 14(3): e82.

Leitlein, L., Smit, E.S., de Vries, H., Hoving, C. Factors influencing Dutch practice nurses' intention to adopt the new smoking cessation intervention PAS, Journal of Advanced Nursing, in press.

Smit, E.S., Evers, S., Hoving, C., de Vries, H. Cost-effectiveness and cost-utility of a web-based multiple tailored smoking cessation programme and tailored counselling by practice nurses, submitted.

Smit, E.S., de Vries, H., Hoving, C. Determinants of the intention to implement a new smoking cessation intervention in Dutch general practices, submitted.

Smit, E.S., Candel, M.J.J.M., Hoving, C., de Vries, H. Who benefits most? A randomized controlled trial testing the long-term effectiveness of an Internet-based smoking cessation programme among subgroups of smokers, submitted. 


\section{Additional publications}

Vangeli, E., Stapleton, J., Smit, E.S., Borland, R., West, R. (2011). Predictors of quit attempts and success of quit attempts in adult general population samples: A systematic review, Addiction, 106(12), 2110-2121.

Smit, E.S., Hoving, C., Cox, V.C.M., de Vries, H. Het bereik van een online advies-opmaat programma voor stoppen met roken: de rol van de praktijkondersteuner, in press.

Prenger, R., Pieterse, M.E., Braakman-Jansen, L.M.A., Feenstra, T.L., Smit, E.S., Hoving, C.; de Vries, H., van Ommeren, J., Evers, S., van der Palen, J., Dealing with delayed behavioral effects in health promotion research by modeling cognitive parameters in cost-effectiveness analyses of behavioral interventions: a validation study, submitted.

\section{Reports}

Willems, R.A., Willemsen, M.C., Nagelhout, G.E., Smit, E.S., Janssen, E., van den Putte, B., de Vries, H. (2012). Evaluatie van de 'Echt stoppen met roken kan met de juiste hulp' campagne, Department of Health Promotion, School for Public Health and Primary Care (CAPHRI), Maastricht University. 